\title{
Cross-Sections of Nanocellulose from Wood Analyzed by Quantized Polydispersity of Elementary Microfibrils
}

\section{Supplementary material}

Tomas Rosén ${ }^{\mathrm{a}, \mathrm{b}, \mathrm{c}}$, HongRui Hea ${ }^{\mathrm{a}}$, Ruifu Wanga ${ }^{\mathrm{a}}$, Chengbo Zhana ${ }^{\mathrm{a}}$, Shirish Chodankard, Andreas Falle, Christian Auline, Per Tomas Larsson $^{\mathrm{c}, \mathrm{e}}$, Tom Lindström ${ }^{\mathrm{a}}$, and Benjamin S. Hsiao ${ }^{\mathrm{a}, 1}$

a Department of Chemistry, Stony Brook University, Stony Brook, NY 11794-3400, USA

${ }^{b}$ Fiber and Polymer Technology, KTH Royal Institute of Technology, SE-100 44, Stockholm, Sweden

${ }^{c}$ Wallenberg Wood Science Center, KTH Royal Institute of Technology, SE-100 44, Stockholm, Sweden

d National Synchrotron Light Source II, Brookhaven National Lab, Upton, NY 11793, USA

e RISE, Box 5604, 114 86, Stockholm, Sweden

${ }^{1}$ To whom correspondence should be addressed 


\section{Contents:}

- TEM images of the 18 CNF samples in the study

- Assessment of isotropy in WAXD experiments

- Results from WAXD simulations

Additionally, a supplementary movie is provided to illustrate the principle of the WAXD simulations. 
TEM images of CNF samples 


\section{TEMPO-oxidized CNF (low charge)}

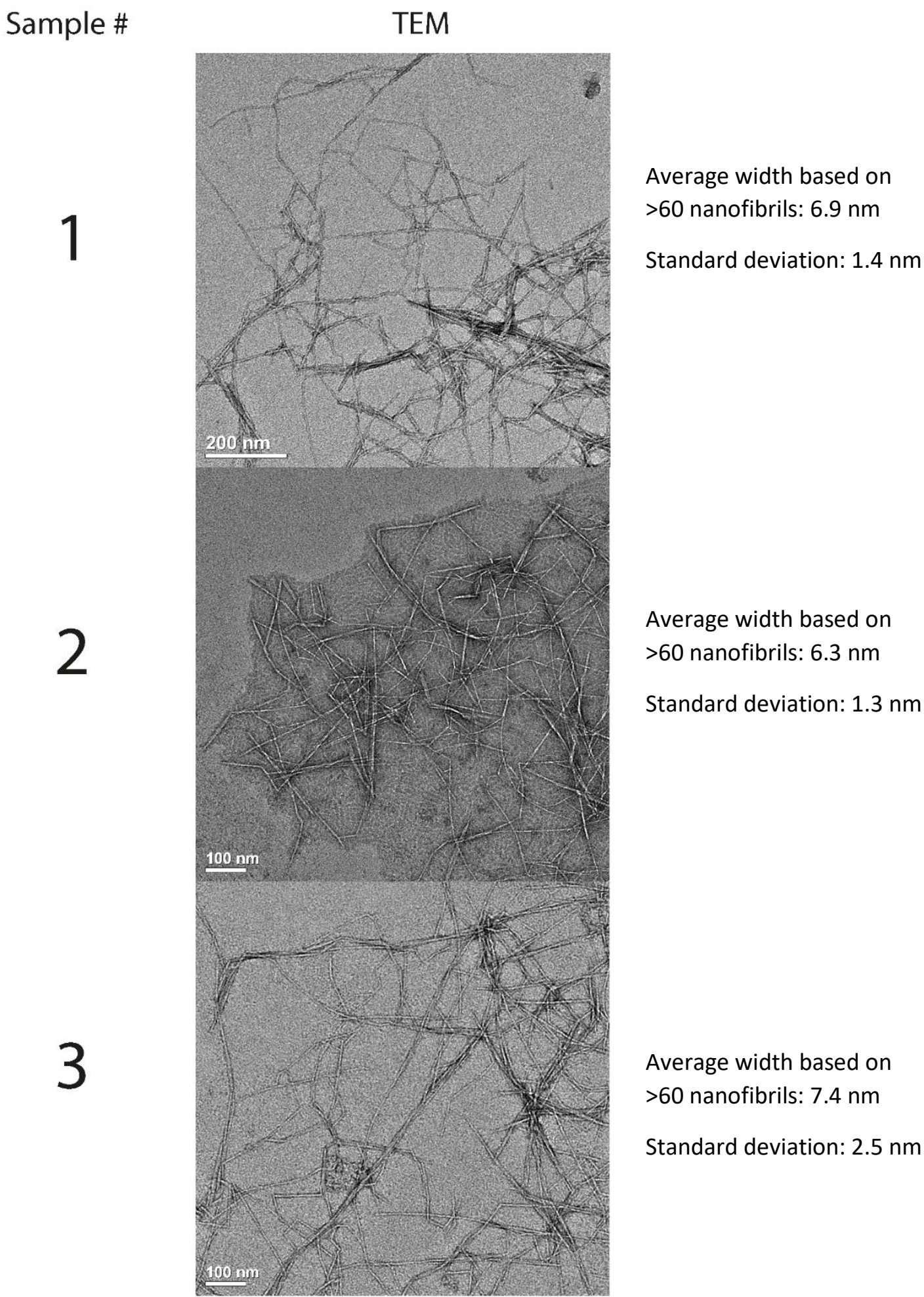




\section{TEMPO-oxidized CNF (high charge)}

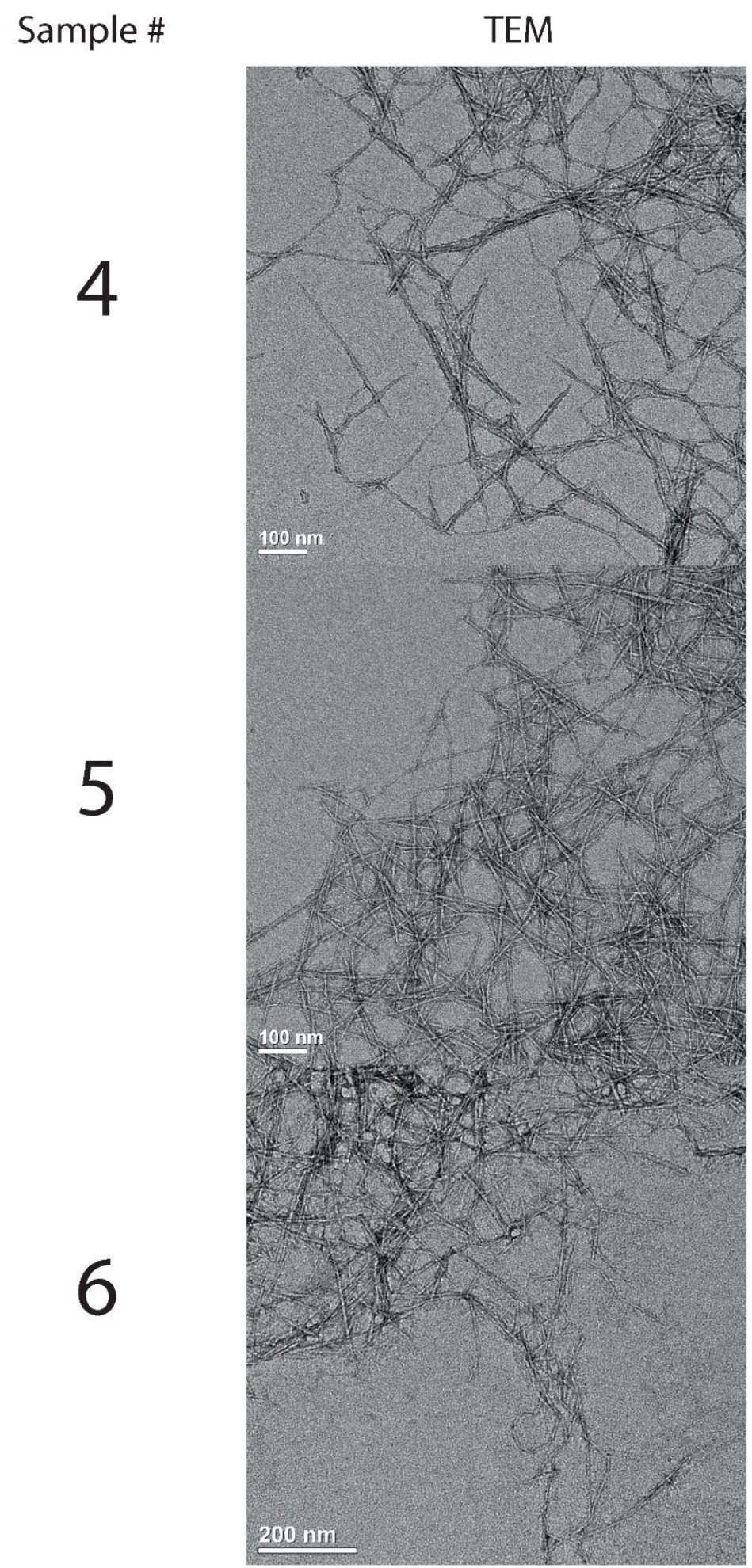

Average width based on $>60$ nanofibrils: $6.9 \mathrm{~nm}$

Standard deviation: $2.3 \mathrm{~nm}$

Average width based on $>60$ nanofibrils: $6.0 \mathrm{~nm}$

Standard deviation: $2.4 \mathrm{~nm}$

Average width based on $>60$ nanofibrils: $7.1 \mathrm{~nm}$

Standard deviation: $1.5 \mathrm{~nm}$ 


\section{Carboxymethylated CNF (lowest charge)}

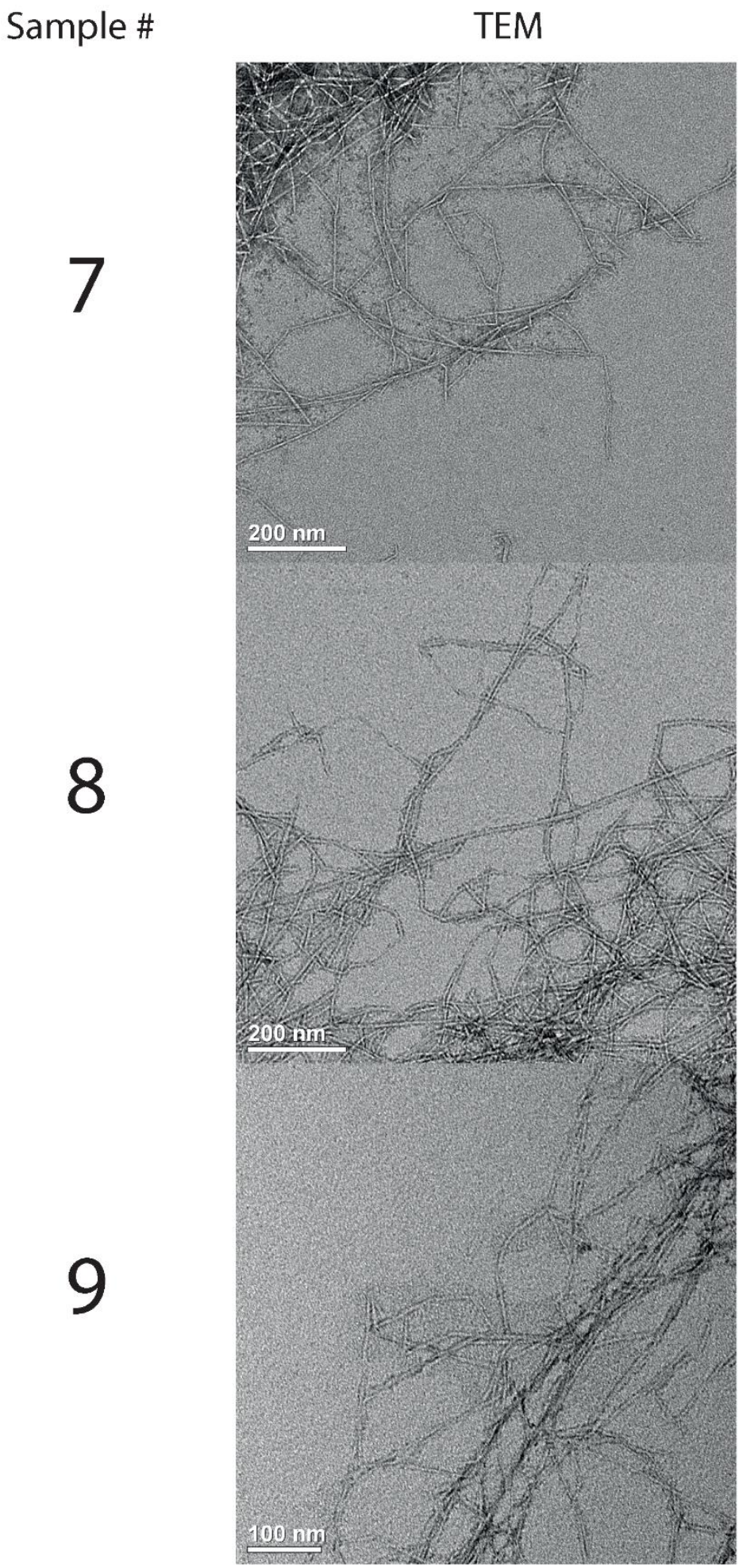

Average width based on $>60$ nanofibrils: $6.2 \mathrm{~nm}$

Standard deviation: $1.1 \mathrm{~nm}$

Average width based on $>60$ nanofibrils: $5.9 \mathrm{~nm}$ Standard deviation: $1.1 \mathrm{~nm}$

Average width based on $>60$ nanofibrils: $6.1 \mathrm{~nm}$ Standard deviation: $1.2 \mathrm{~nm}$ 


\section{Carboxymethylated CNF (low charge)}

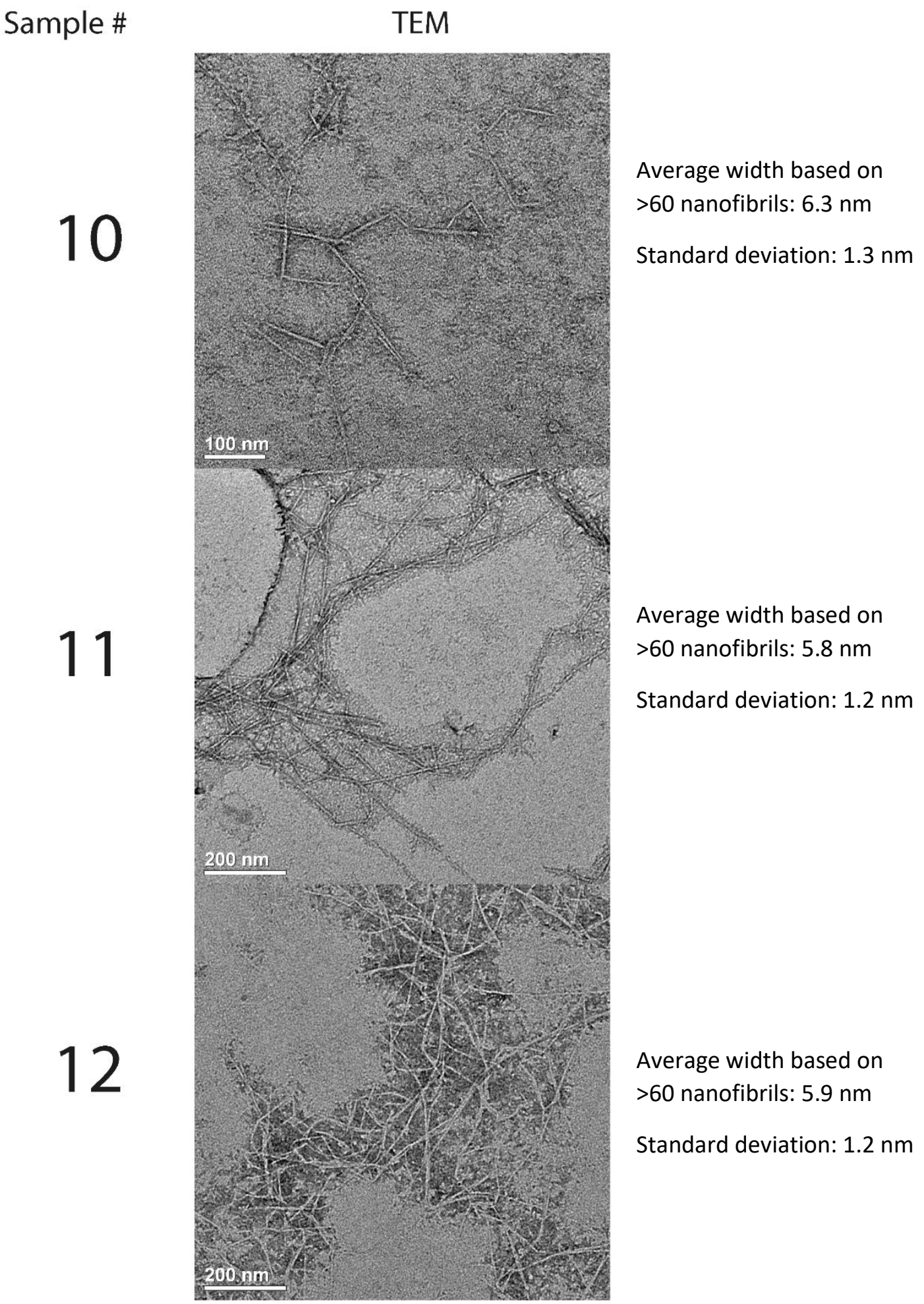




\section{Carboxymethylated CNF (high charge)}

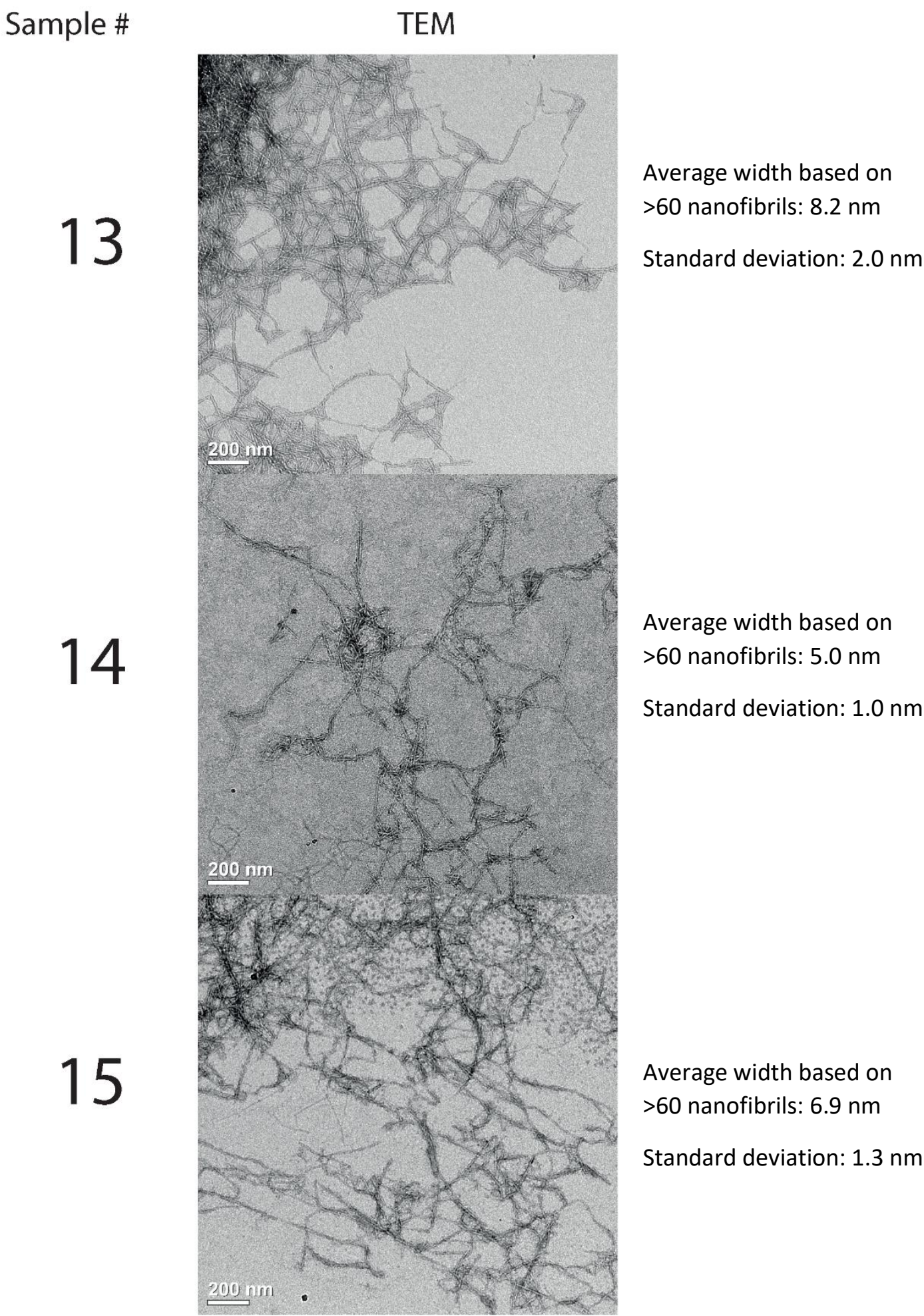




\section{Carboxymethylated CNF (highest charge)}

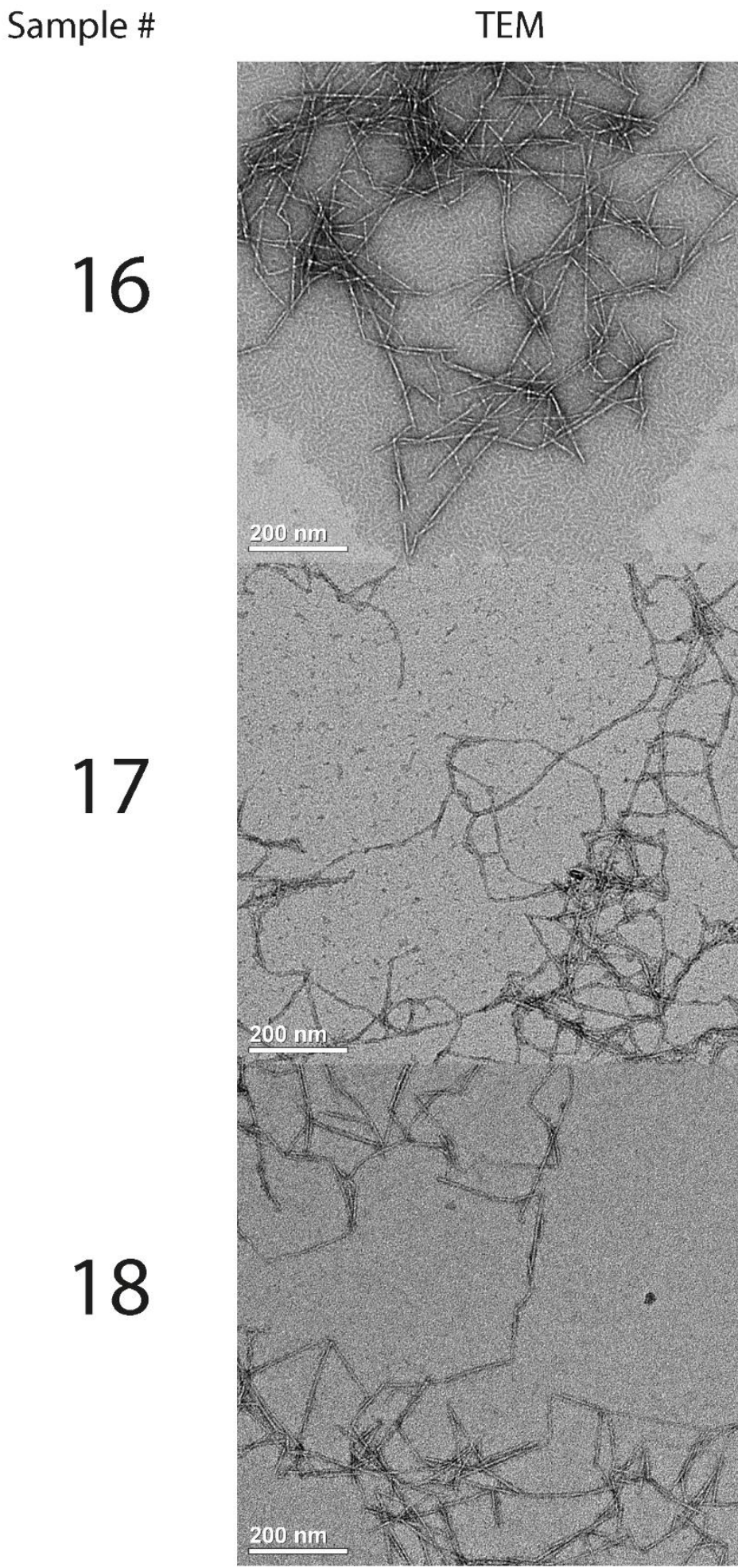

Average width based on $>60$ nanofibrils: $7.7 \mathrm{~nm}$

Standard deviation: $1.8 \mathrm{~nm}$

Average width based on $>60$ nanofibrils: $6.8 \mathrm{~nm}$

Standard deviation: $2.0 \mathrm{~nm}$

Average width based on $>60$ nanofibrils: $6.1 \mathrm{~nm}$

Standard deviation: $1.2 \mathrm{~nm}$ 
WAXD experiments: ensuring isotropy of samples 


\section{Procedure:}

Simultaneous SAXS and WAXD signals were recorded on 3 different detectors (average of 5 different measurement locations, each with $1 \mathrm{~s}$ exposure):

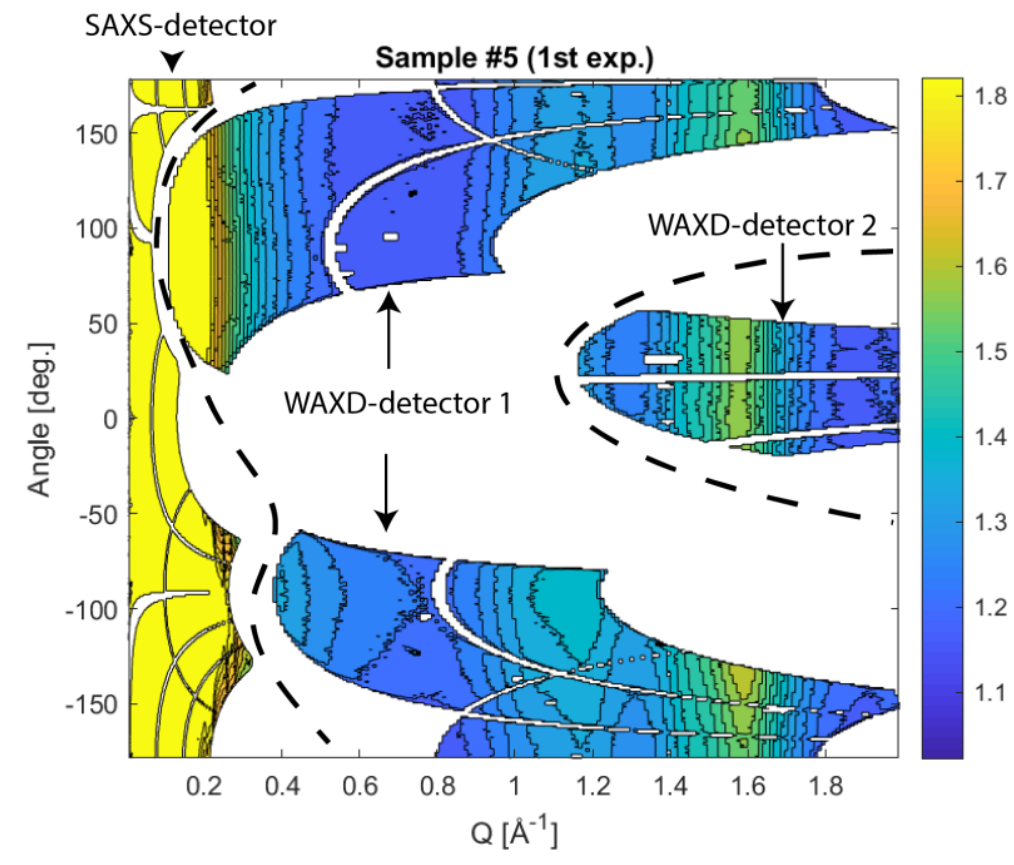

The in-accessible (zero) pixels at an angle $\chi$ are corrected with data from the corresponding mirrorpixel (if accessible) at $\chi \pm 180^{\circ}$, due to symmetry:

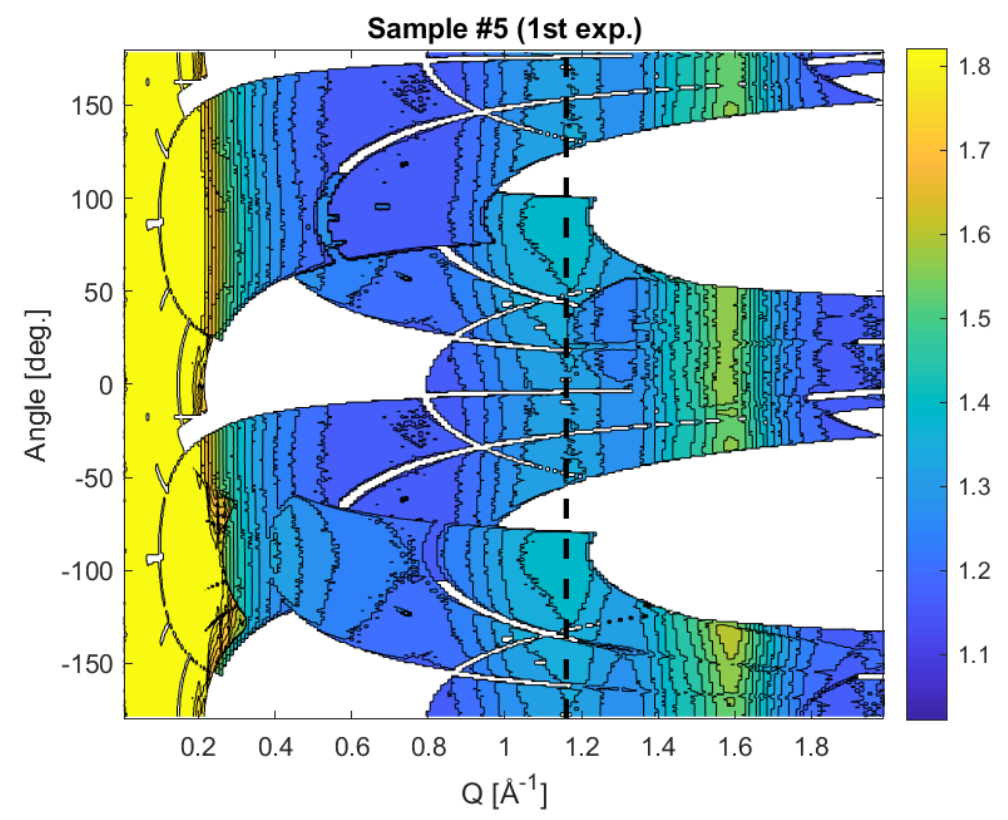

Note that this mirror-correction does not lead to a perfect matching owing to differences in the intensity due to slight detector tilt. The orientation distribution is evaluated at the location of the (110)-peak at $q=1.16 \AA^{-1}$ (thick dashed black line; note that the data at this q-value is only taken from one detector, i.e. WAXD-detector 1$)$ : 


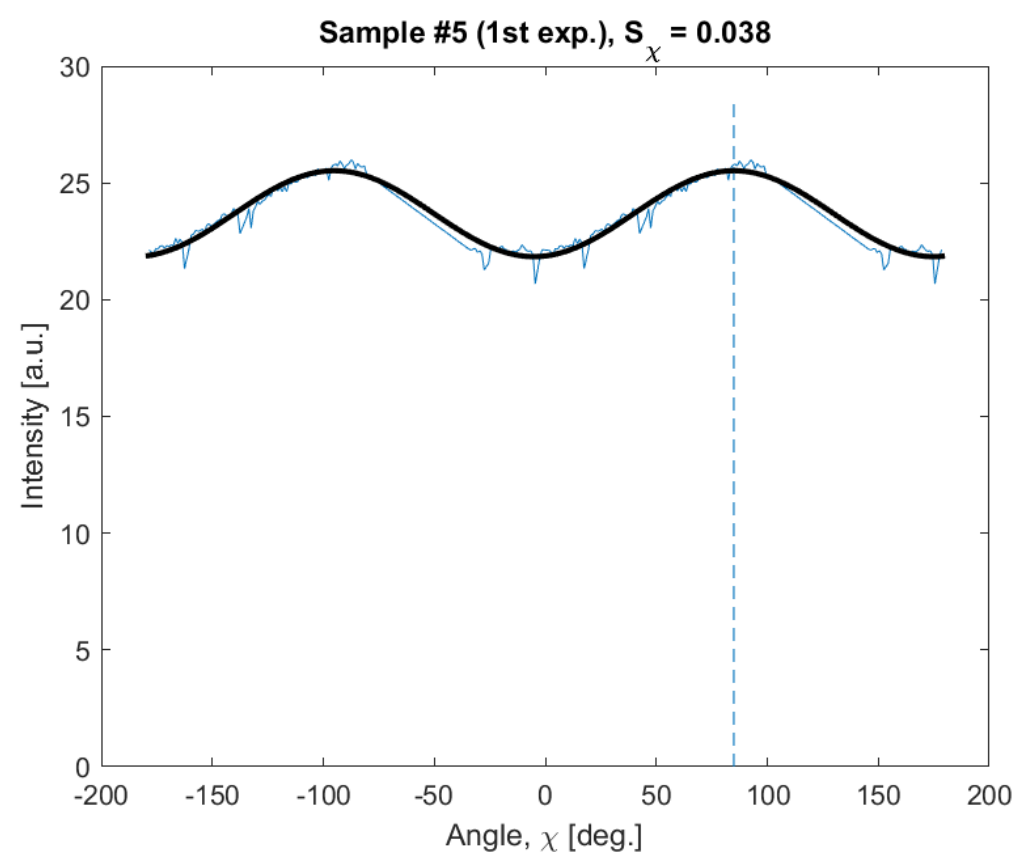

The raw intensity $I(\chi)$ is fitted to a function of form:

$I_{f i t}(\chi)=A \cos \left(2\left(\chi-\chi_{\text {tilt }}\right)\right)+B$,

where $\chi_{\text {tilt }}$ indicates the tilt angle (dashed blue line in figure). The order parameter $S_{\chi}$ is calculated through:

$S_{\chi}=\int_{-180^{\circ}}^{180^{\circ}} I_{f i t}(\chi)\left(2 \cos ^{2}\left(\chi-\chi_{t i l t}\right)-1\right) d \chi$,

Where $I_{f i t}(\chi)$ initially is normalized according to:

$1=\int_{-180^{\circ}}^{180^{\circ}} I_{f i t}(\chi) d \chi$ 


\section{Results:}

The order parameter of all samples in the study are presented in the table below. Note that every sample was measured in two separate experiments (at different locations).

\begin{tabular}{|l|c|c|}
\hline Sample \# & $S_{\chi}\left(^{\text {st }}\right.$ exp. $)$ & $S_{\chi}$ (2 $^{\text {nd }}$ exp. $)$ \\
\hline 1 & 0.023 & 0.014 \\
\hline 2 & 0.029 & 0.022 \\
\hline 3 & 0.008 & 0.016 \\
\hline 4 & 0.016 & 0.042 \\
\hline 5 & 0.038 & 0.020 \\
\hline 6 & 0.007 & 0.009 \\
\hline 7 & 0.002 & 0.039 \\
\hline 8 & 0.027 & 0.024 \\
\hline 9 & 0.014 & 0.016 \\
\hline 10 & 0.006 & 0.011 \\
\hline 11 & 0.018 & 0.051 \\
\hline 12 & 0.016 & 0.029 \\
\hline 13 & 0.025 & 0.025 \\
\hline 14 & 0.008 & 0.046 \\
\hline 15 & 0.015 & 0.015 \\
\hline 16 & 0.032 & 0.037 \\
\hline 17 & 0.012 & 0.018 \\
\hline 18 & 0.004 & 0.008 \\
\hline
\end{tabular}

Since all the samples have an order parameter that fulfill $S_{\chi} \ll 0.1$, we can safely assume the samples to be isotropic. 


\section{WAXD simulations: Results}

Form factor of a CNF chain with semi-axes

$1 \AA ̊ \times 1 \AA$ 
Model: H1+

Aggregation direction: (1-10)

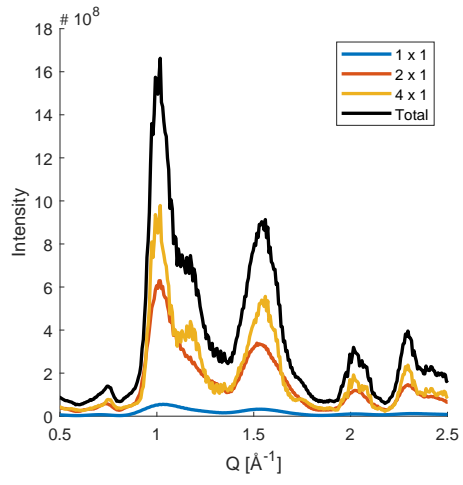

Sample \#1

Vol\% 1x1: 4.7

Vol\% $2 \times 1: 42.3$

Vol\% $4 \times 1: 53.0$
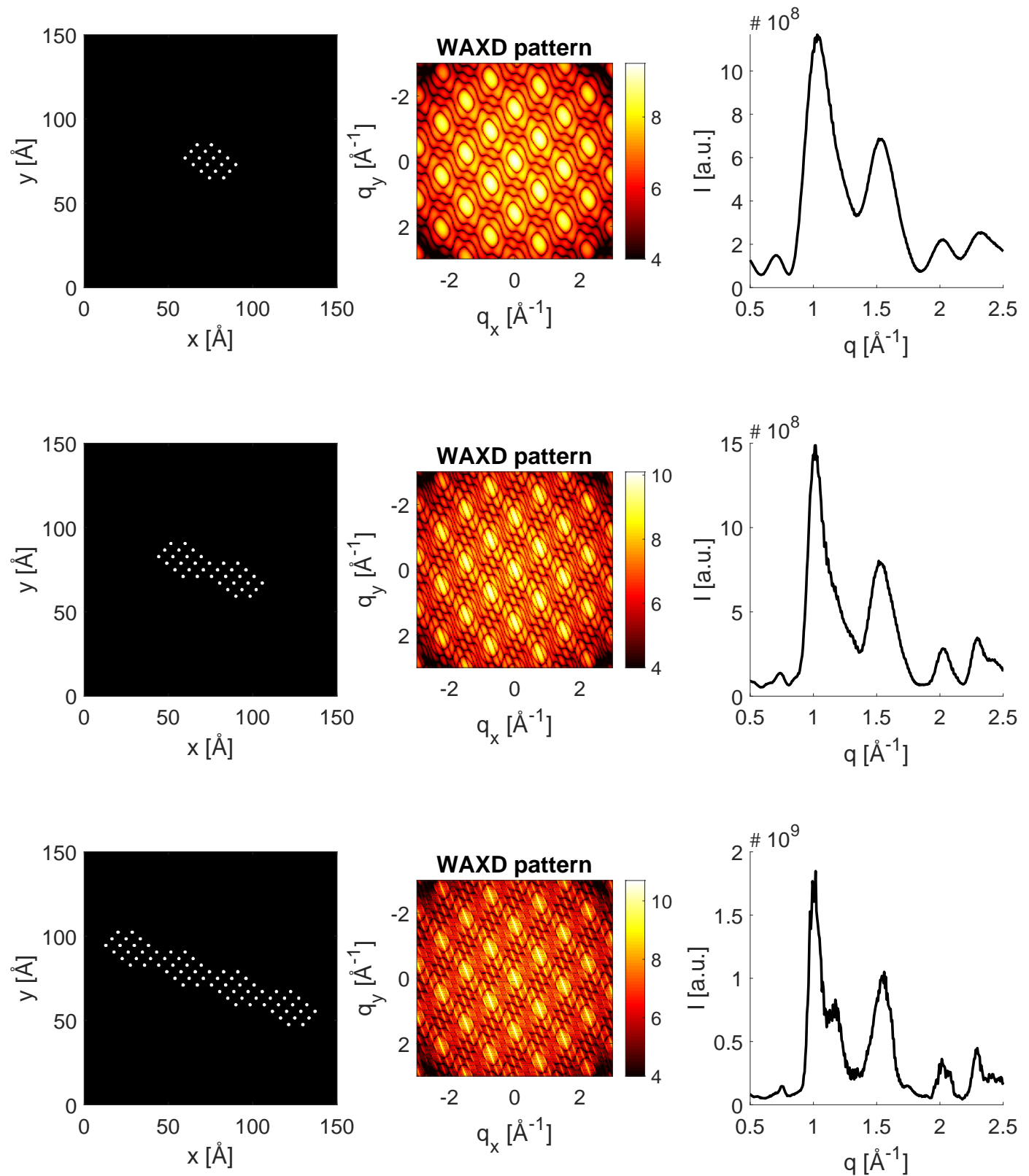
Model: H1+

Aggregation direction: (110)

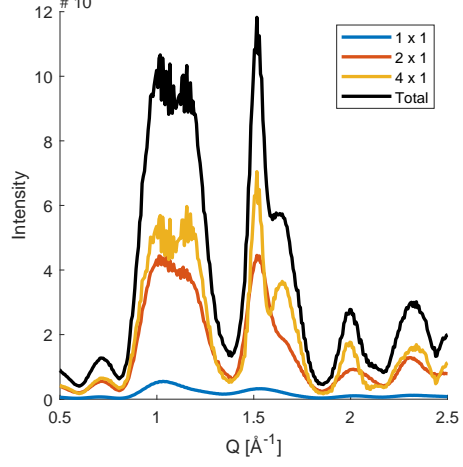

Sample \#1

Vol\% 1x1: 4.7

Vol\% 2x1: 42.3

Vol\% 4x1: 53.0
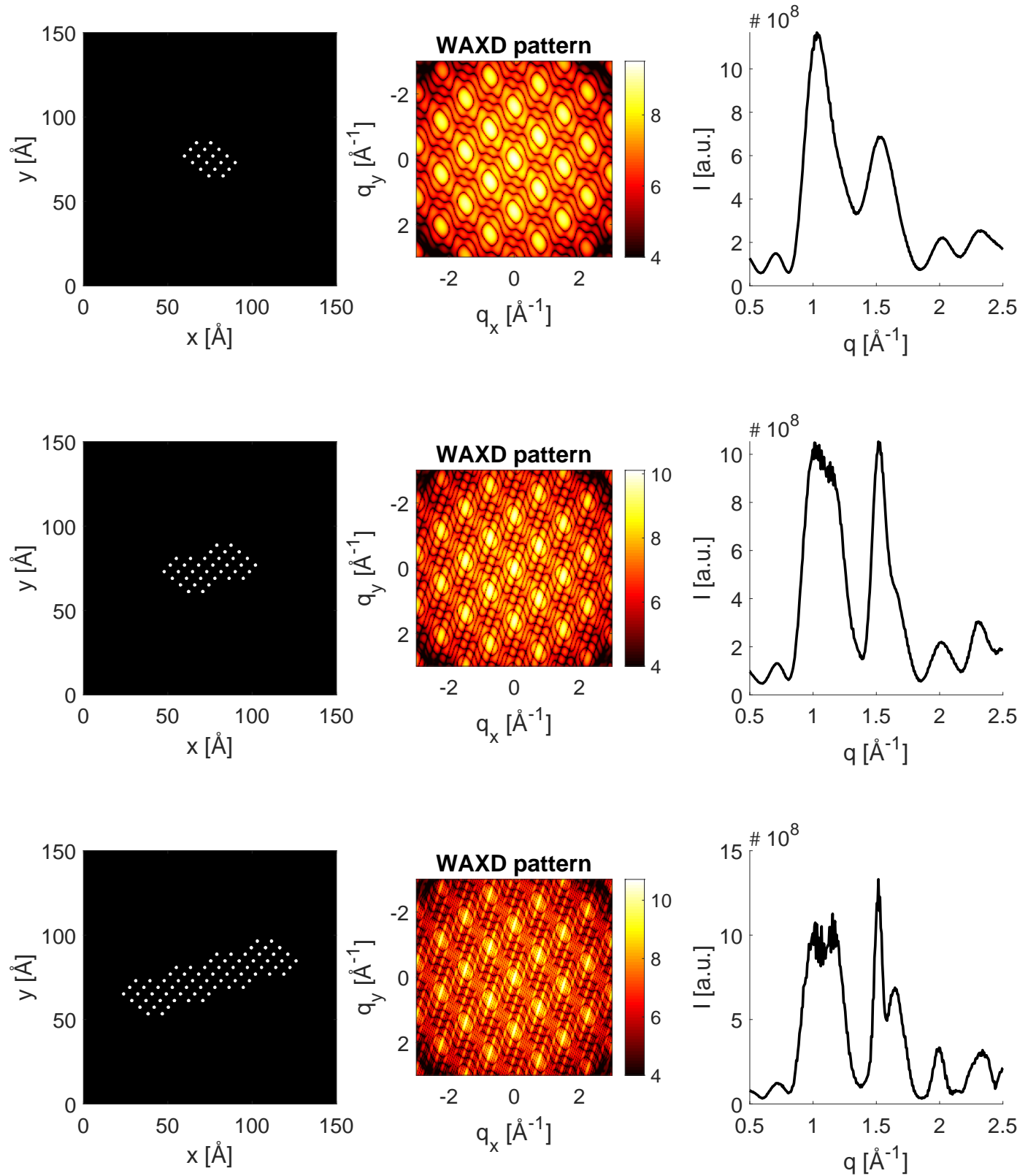
Model: H1+

Aggregation direction: (200)

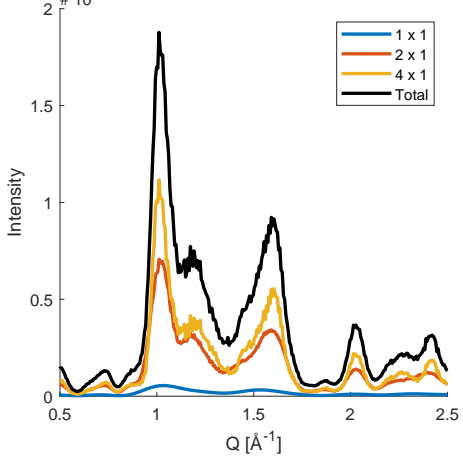

Sample \#1

Vol\% 1x1: 4.7

Vol\% 2x1: 42.3

Vol\% 4x1: 53.0
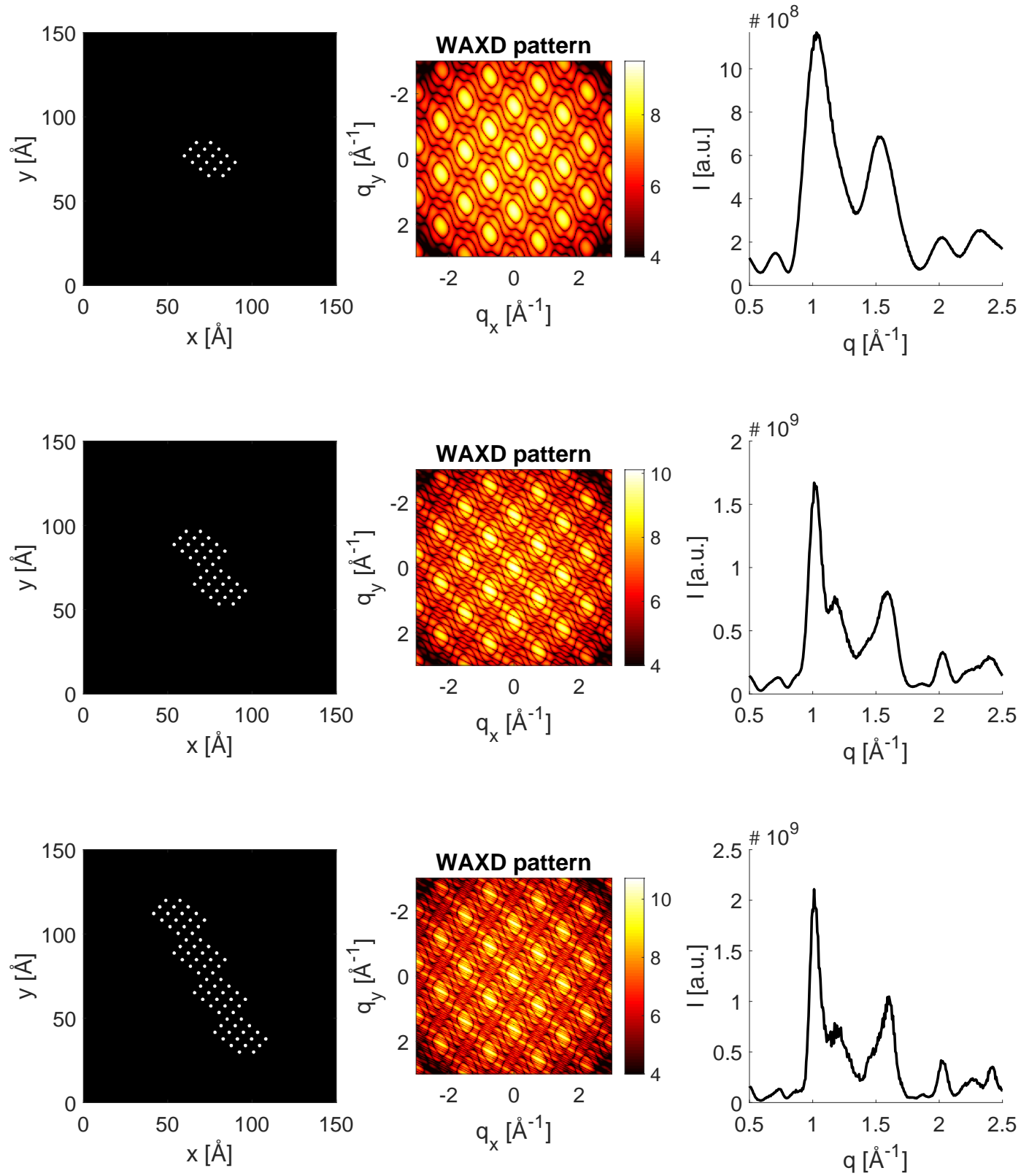
Model: H1-

Aggregation direction: (1-10)

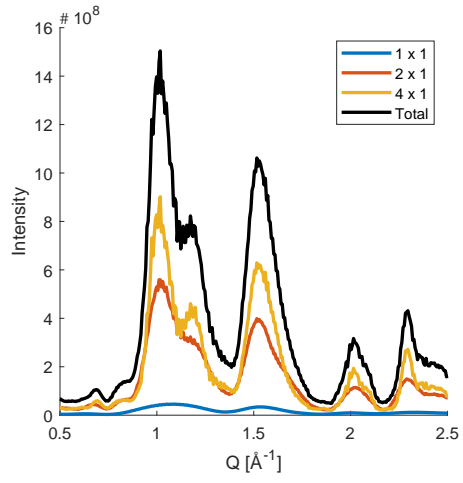

Sample \#1

Vol\% 1x1: 4.7

Vol\% $2 \times 1: 42.3$

Vol\% 4x1: 53.0
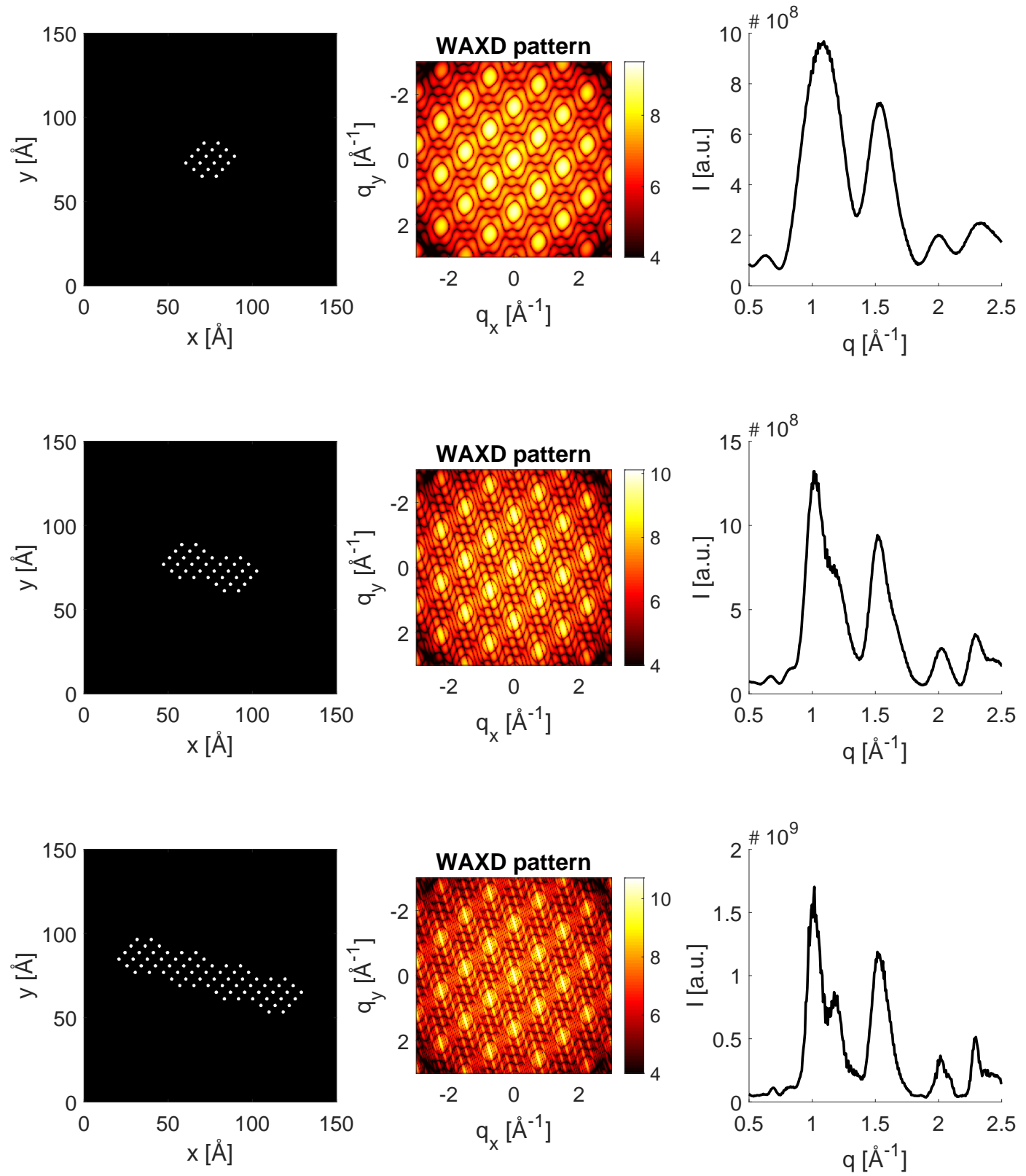
Model: H1-

Aggregation direction: (110)

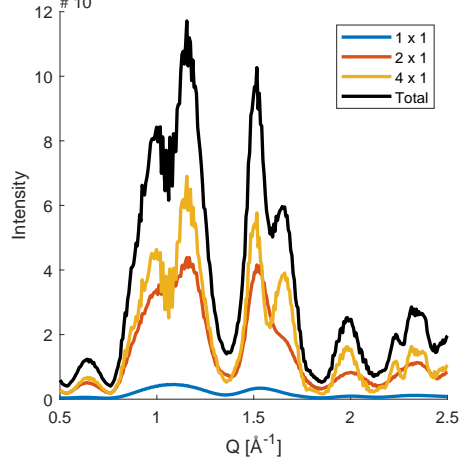

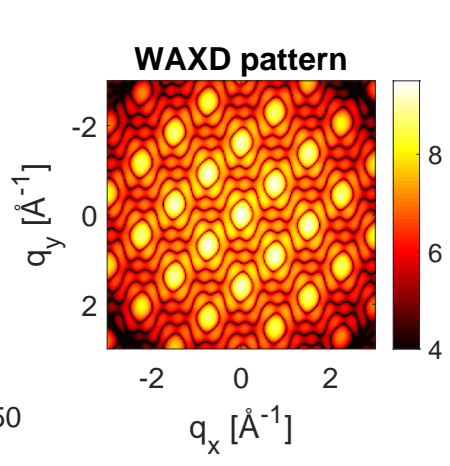
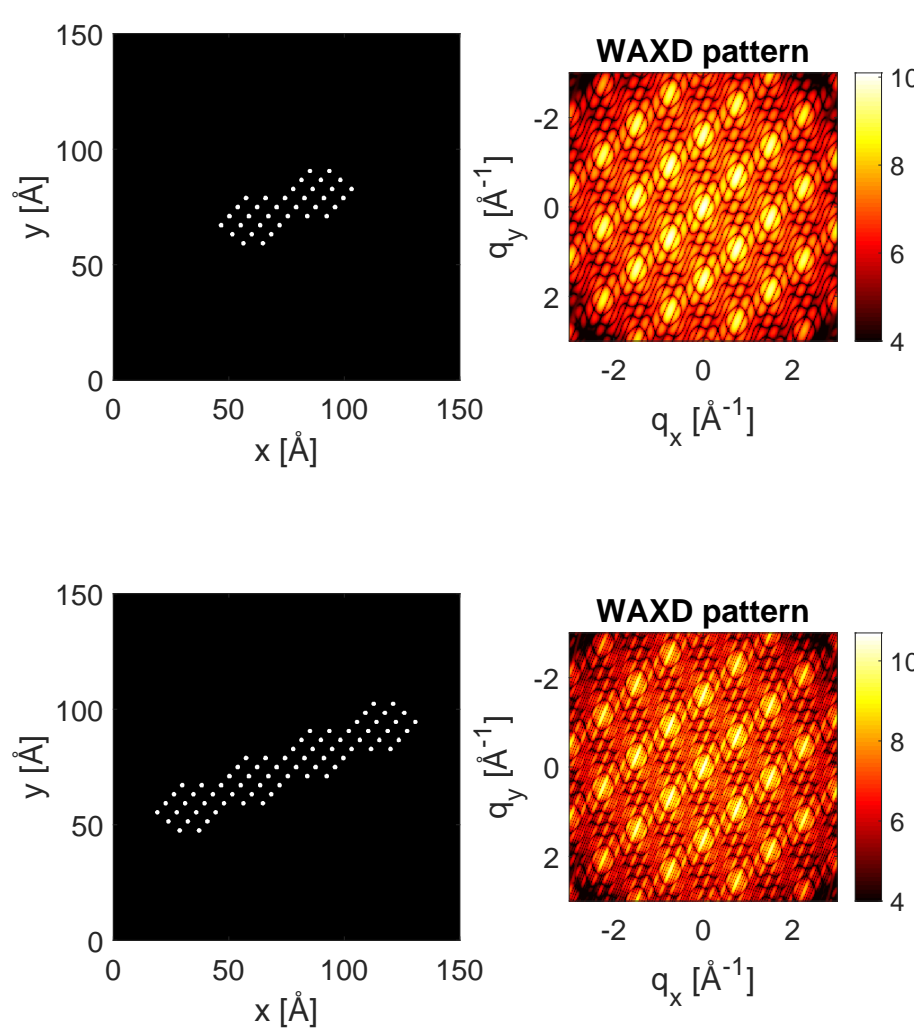

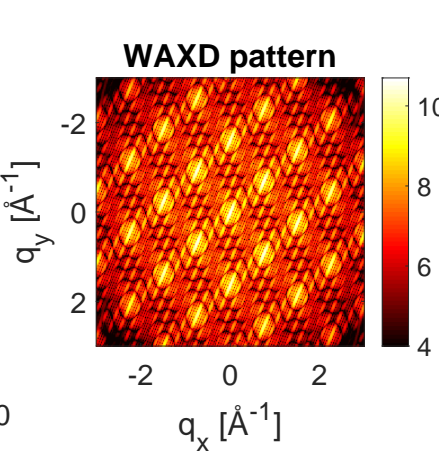

$q_{x}\left[\AA^{-1}\right]$
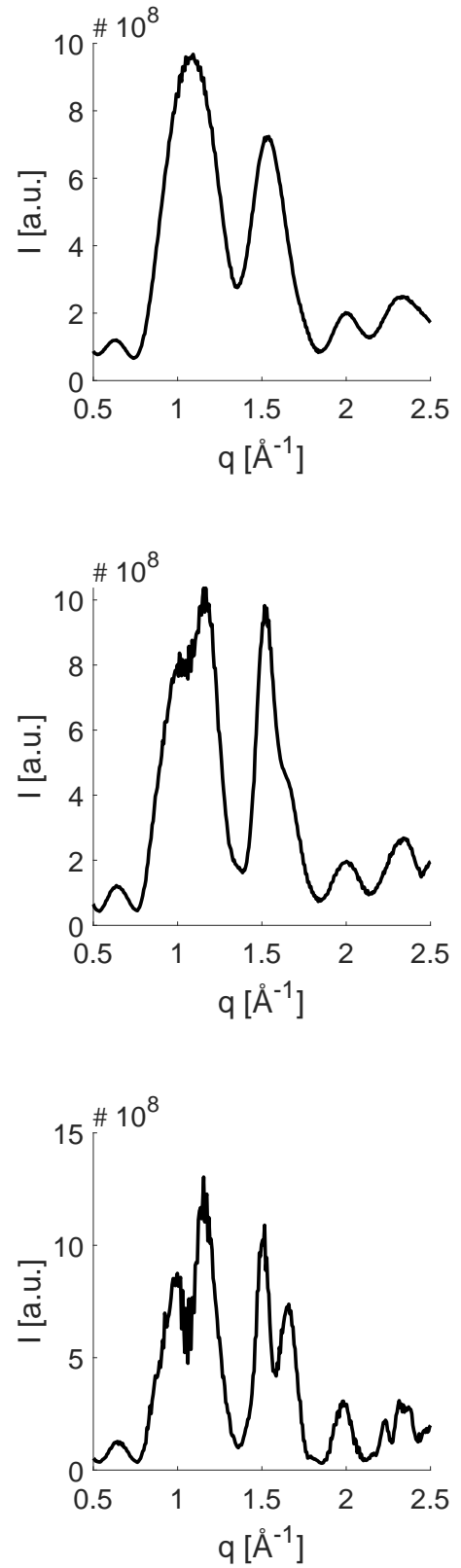
Model: H1-

Aggregation direction: (200)

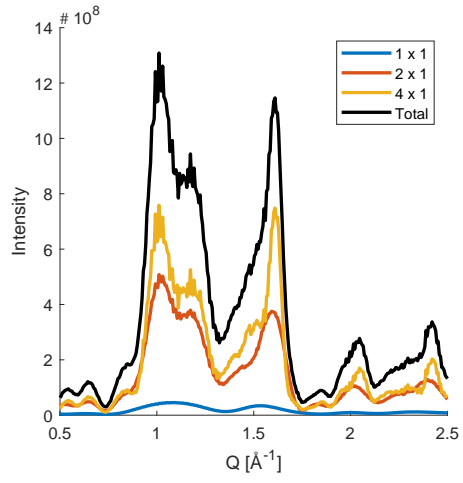

Sample \#1

Vol\% 1x1: 4.7

Vol\% $2 \times 1: 42.3$

Vol\% $4 \times 1: 53.0$
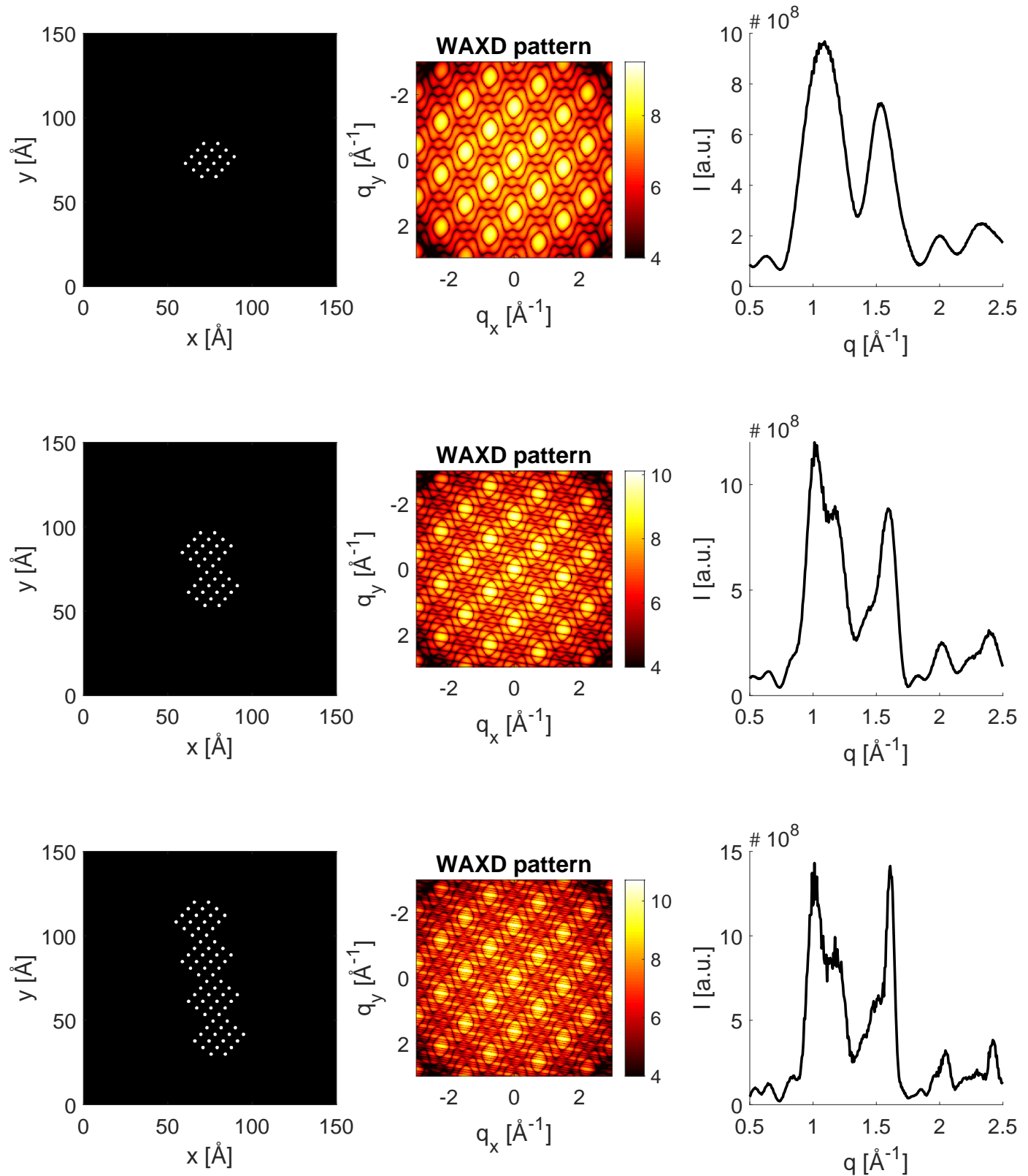
Model: $\mathrm{H} 2+$

Aggregation direction: (1-10)

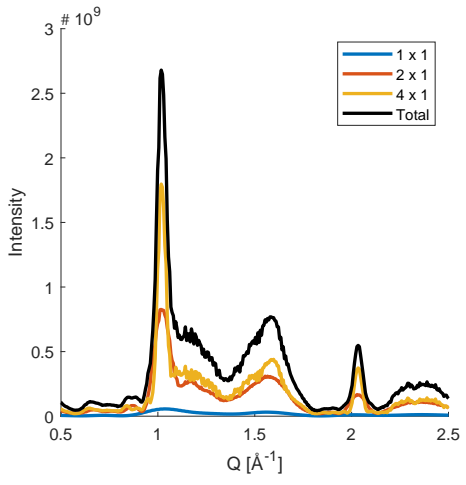

Sample \#1

Vol\% 1x1: 4.7

Vol\% $2 \times 1: 42.3$

Vol\% $4 \times 1: 53.0$
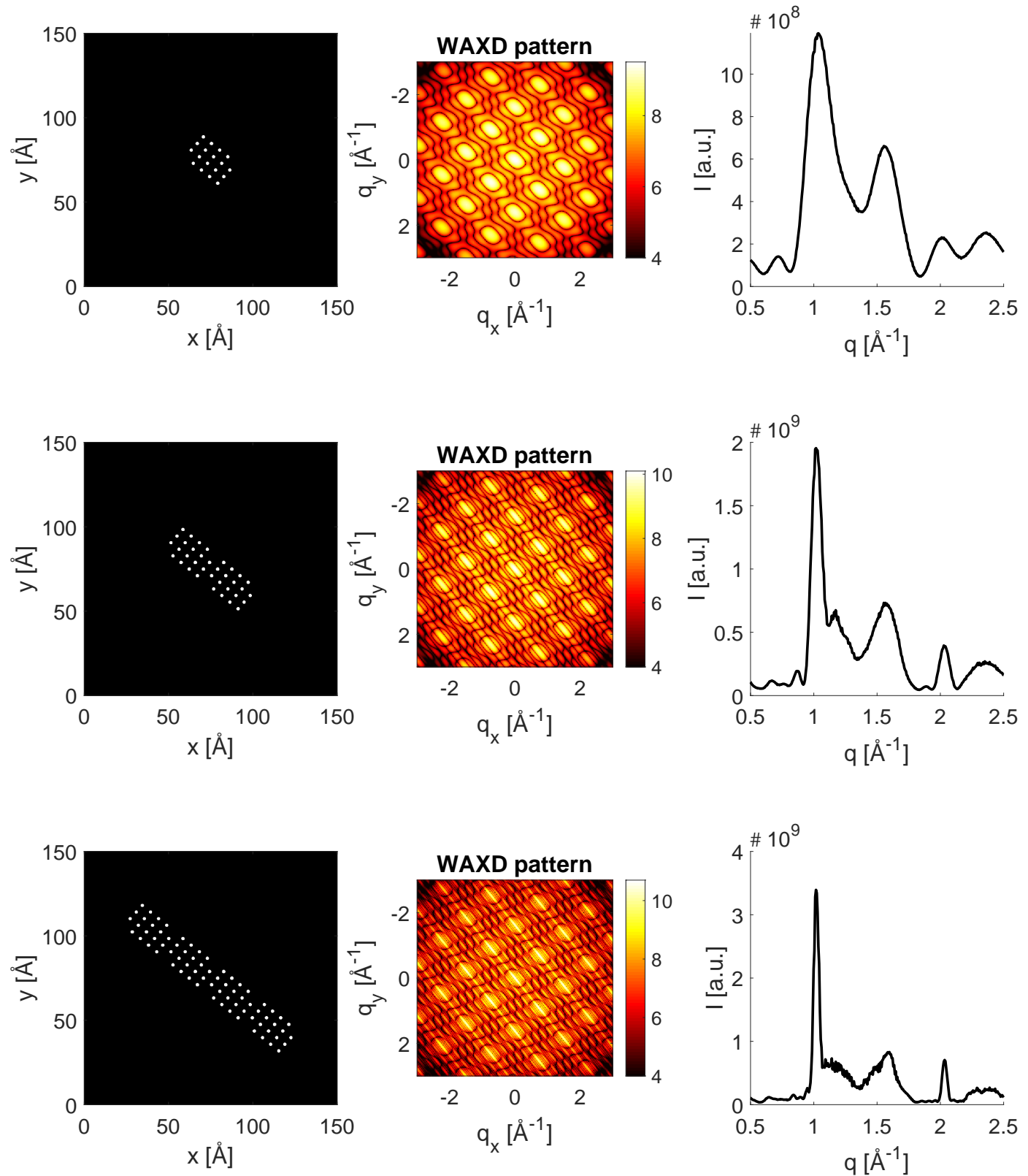
Model: $\mathrm{H} 2+$

Aggregation direction: (110)

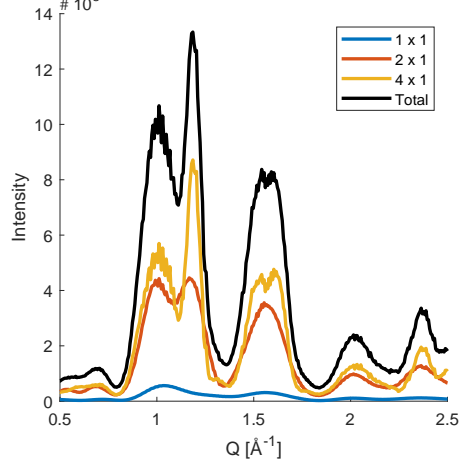

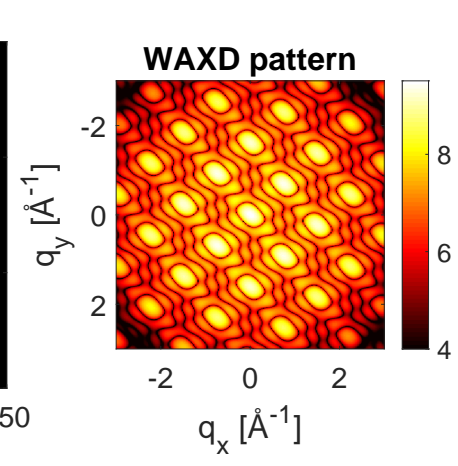
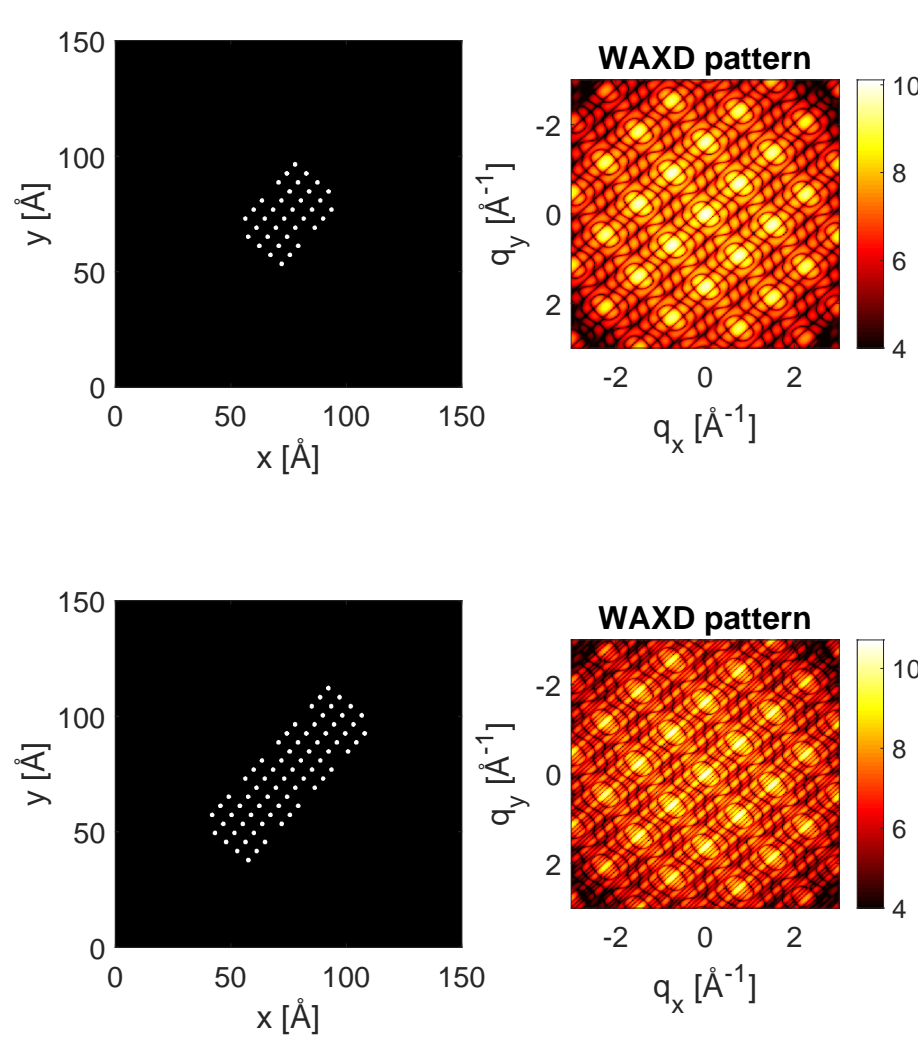

Sample \#1

Vol\% 1 1 1: 4.7

Vol\% $2 \times 1: 42.3$

Vol\% $4 \times 1: 53.0$
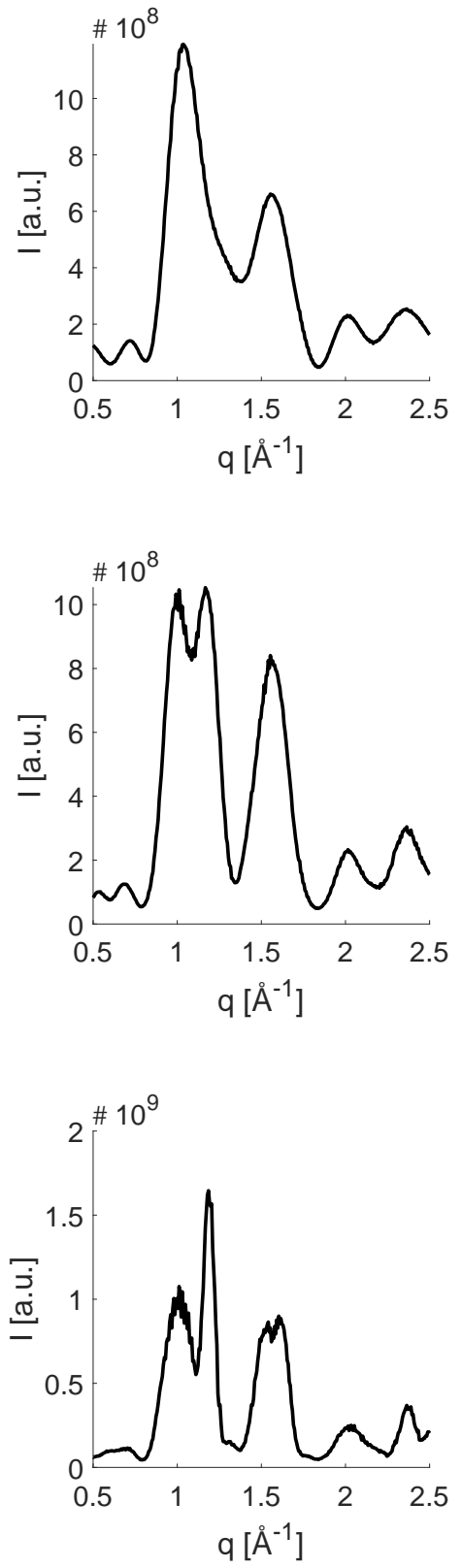
Model: H2-

Aggregation direction: (1-10)

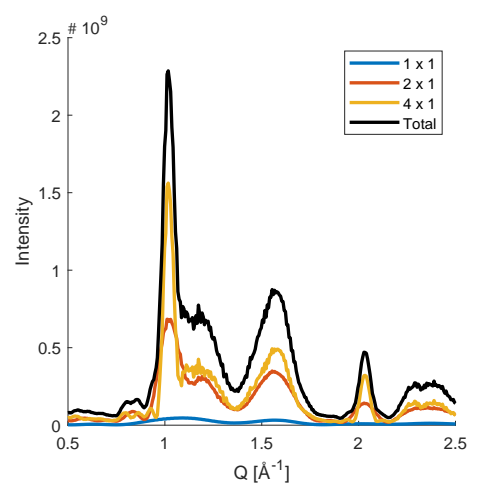

Sample \#1

Vol\% 1x1: 4.7

Vol\% $2 \times 1: 42.3$

Vol\% $4 \times 1: 53.0$
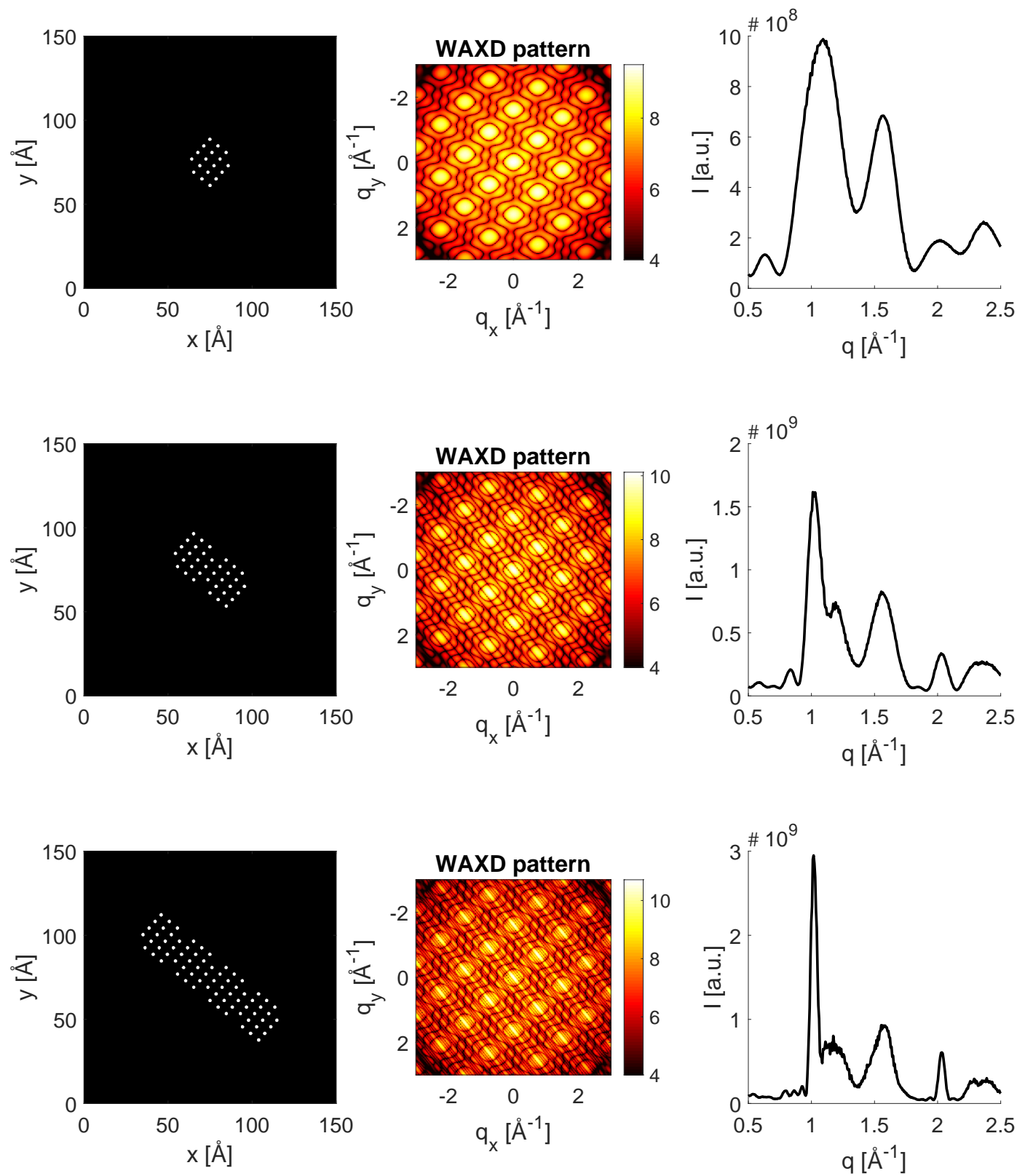
Model: H2-

Aggregation direction: (110)

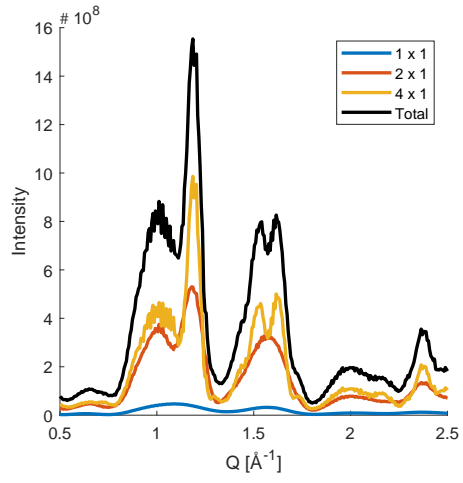

Sample \#1

Vol\% 1x1: 4.7

Vol\% $2 \times 1: 42.3$

Vol\% 4x1: 53.0
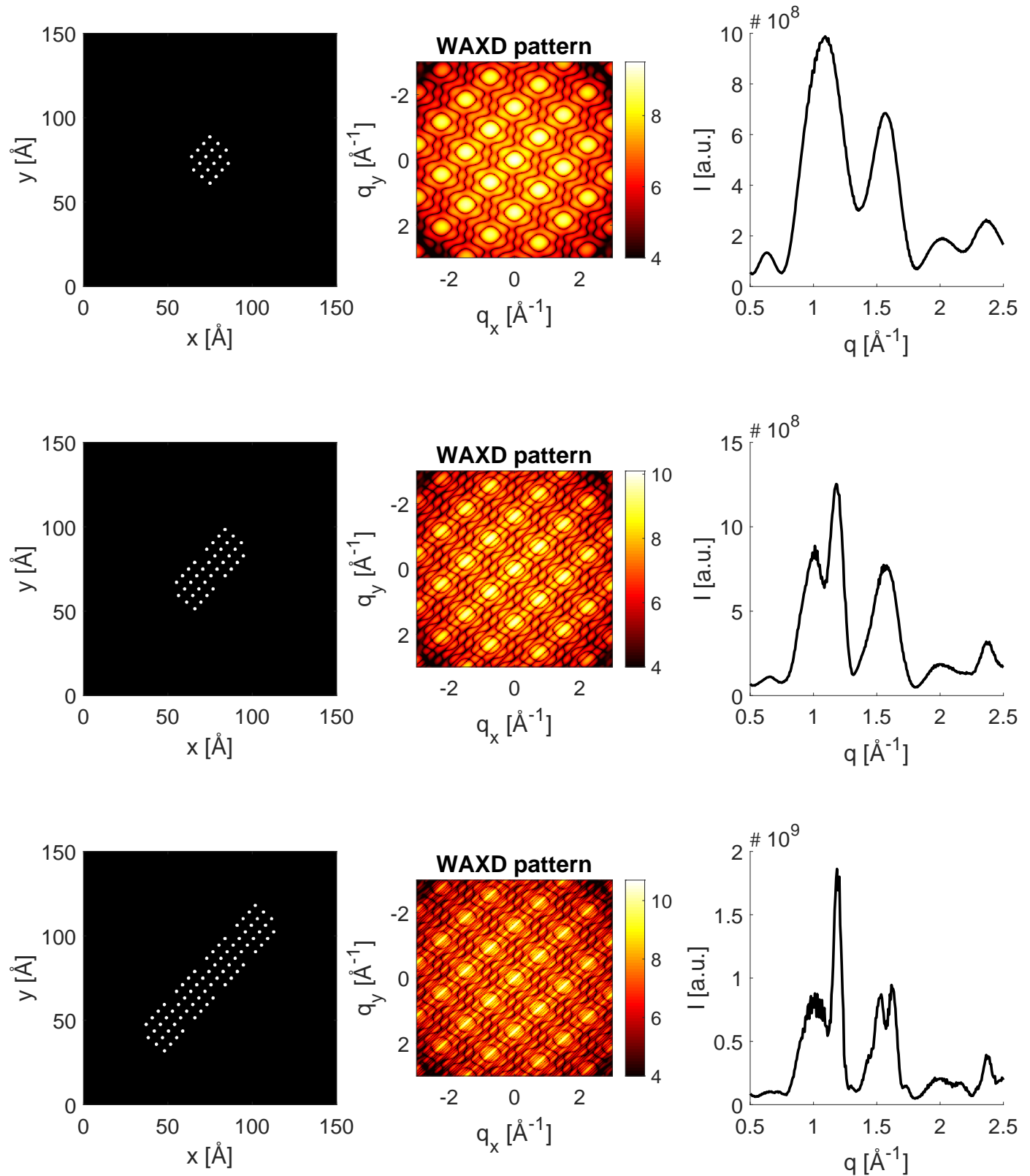
Model: R1+

Aggregation direction: (1-10)

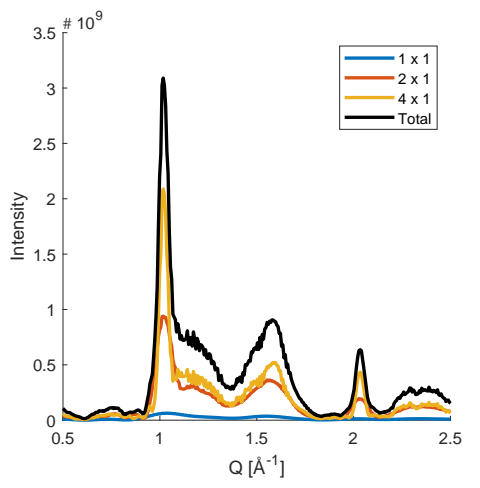

Sample \#1

Vol\% 1x1: 4.7

Vol\% $2 \times 1: 42.3$

Vol\% $4 \times 1: 53.0$
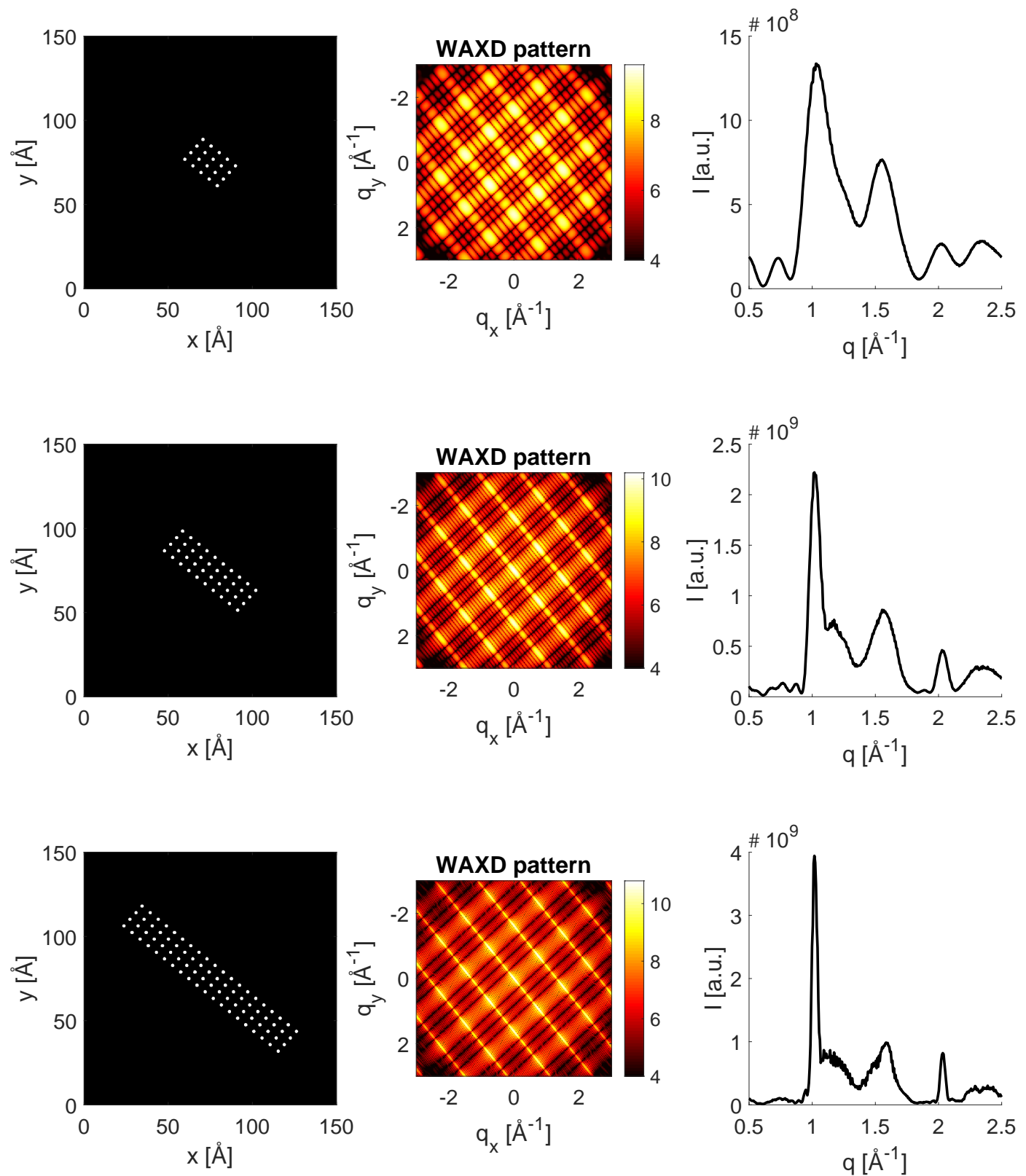
Model: R1+

Aggregation direction: (110)

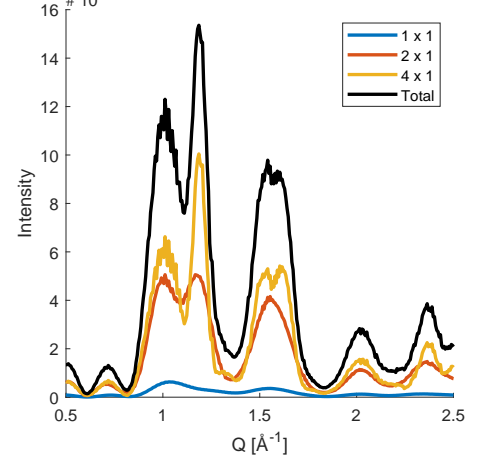

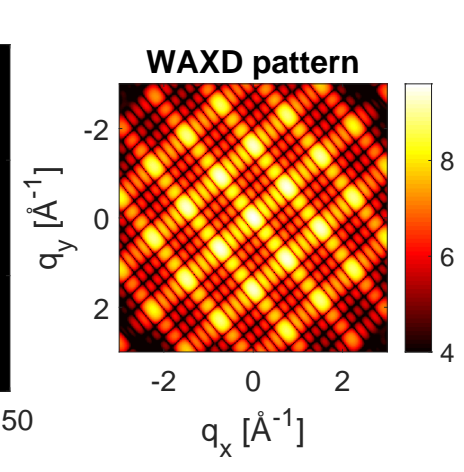

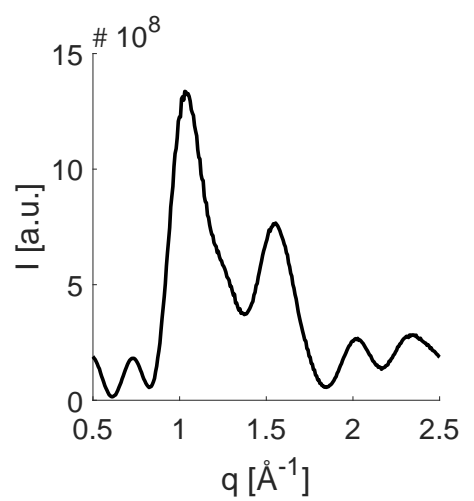

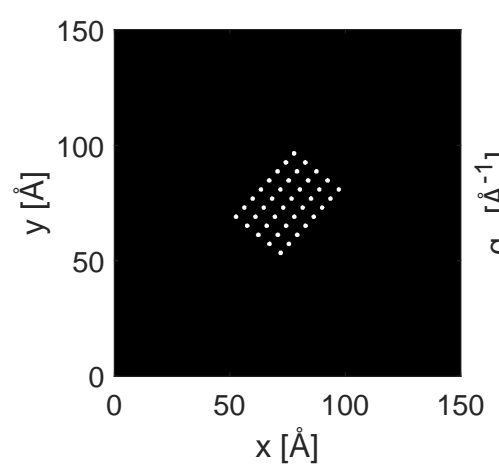
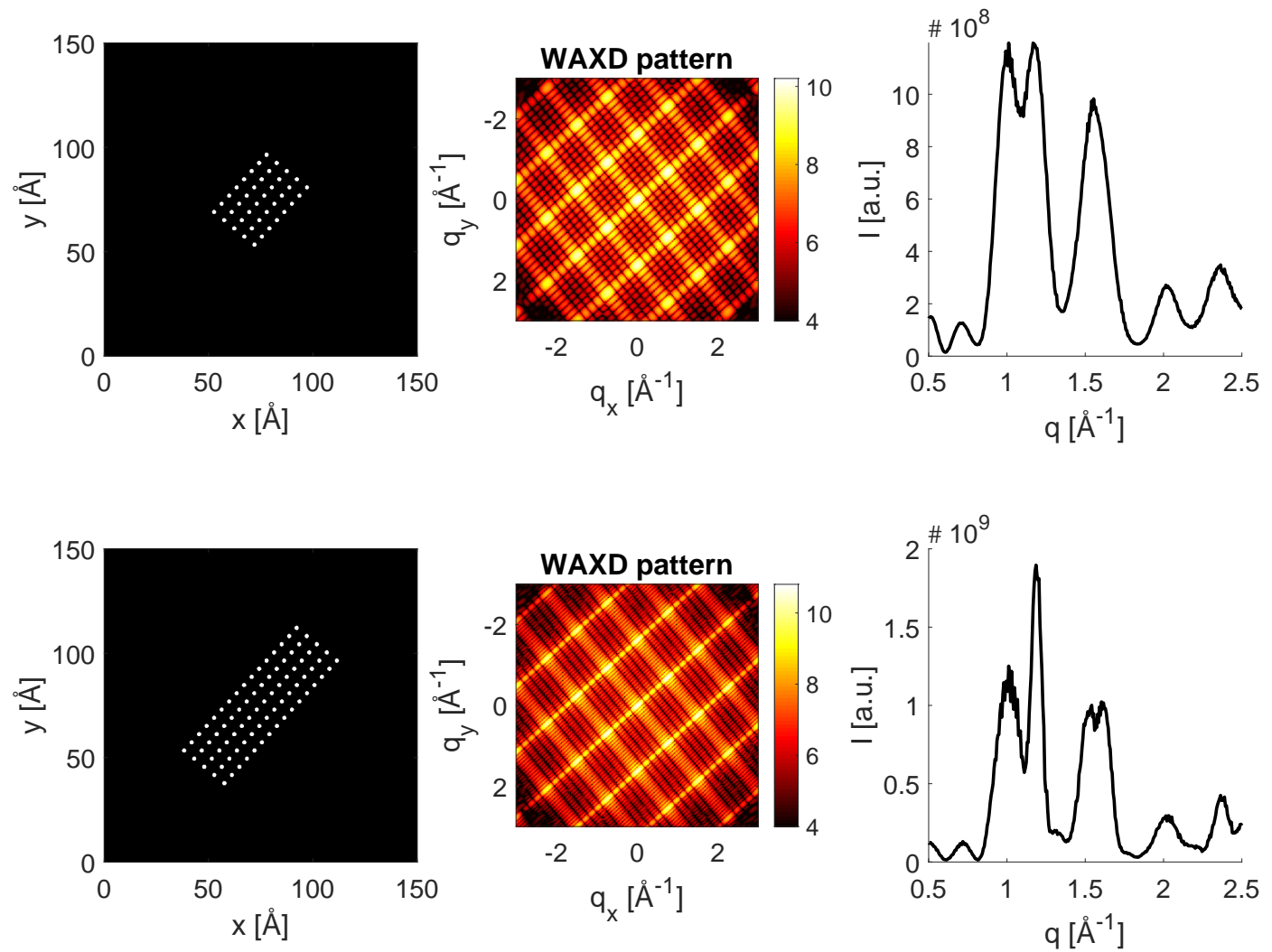

Vol\% $1 \times 1: 4.7$

Vol\% 2x1: 42.3

Vol\% 4x1: 53.0

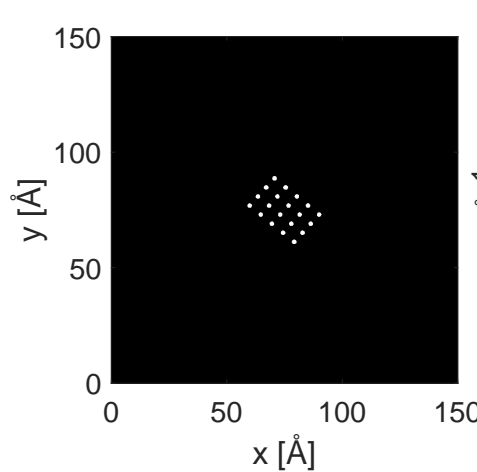

$\mathrm{q}_{\mathrm{X}}[\mathrm{A}]$

$\mathrm{q}\left[\mathrm{A}^{-1}\right]$ 
Model: R1-

Aggregation direction: (1-10)

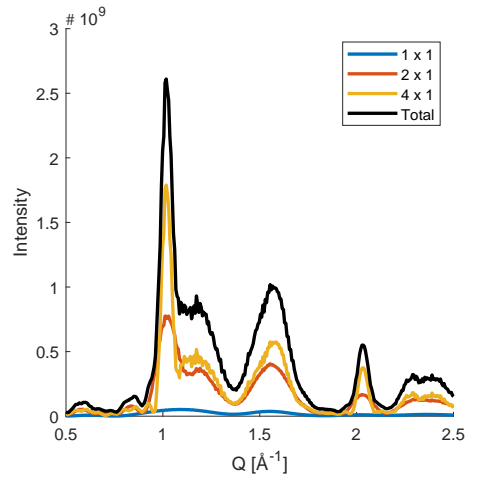

Sample \#1

Vol\% 1x1: 4.7

Vol\% $2 \times 1: 42.3$

Vol\% $4 \times 1: 53.0$
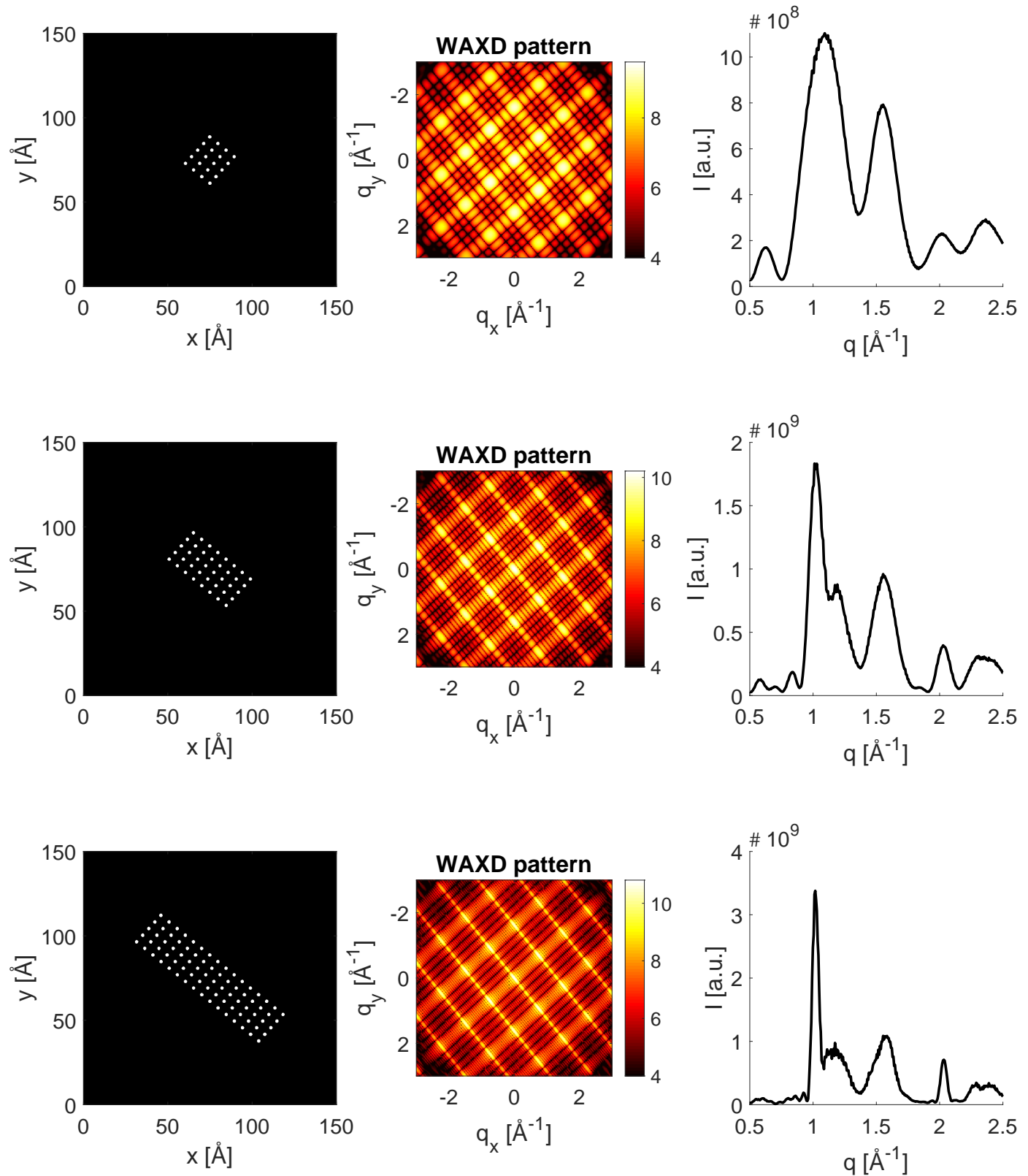
Model: R1-

Aggregation direction: (110)

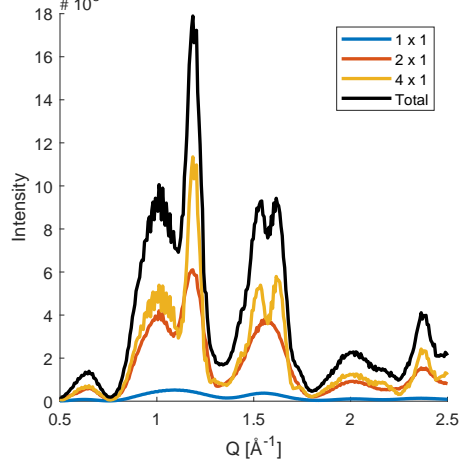

Sample \#1

Vol\% 1x1: 4.7

Vol\% $2 \times 1: 42.3$

Vol\% $4 \times 1: 53.0$
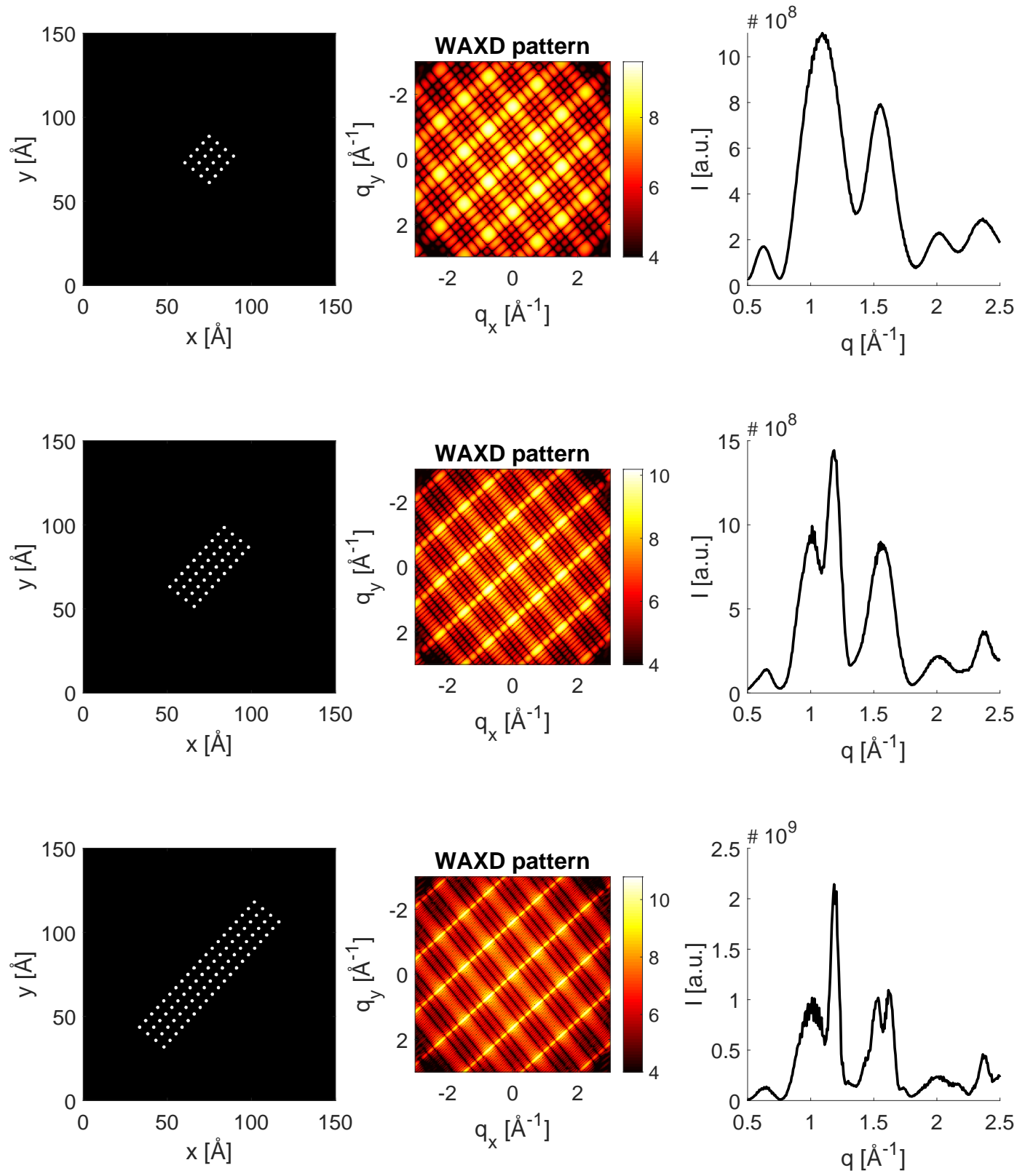
Model: R2+

Aggregation direction: (1-10)

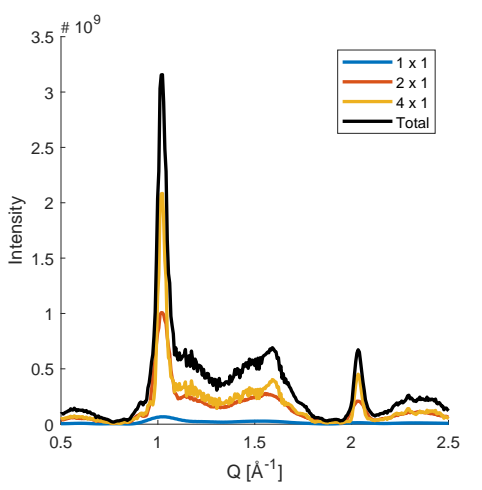

Sample \#1

Vol\% 1x1: 4.7

Vol\% $2 \times 1: 42.3$

Vol\% $4 \times 1: 53.0$
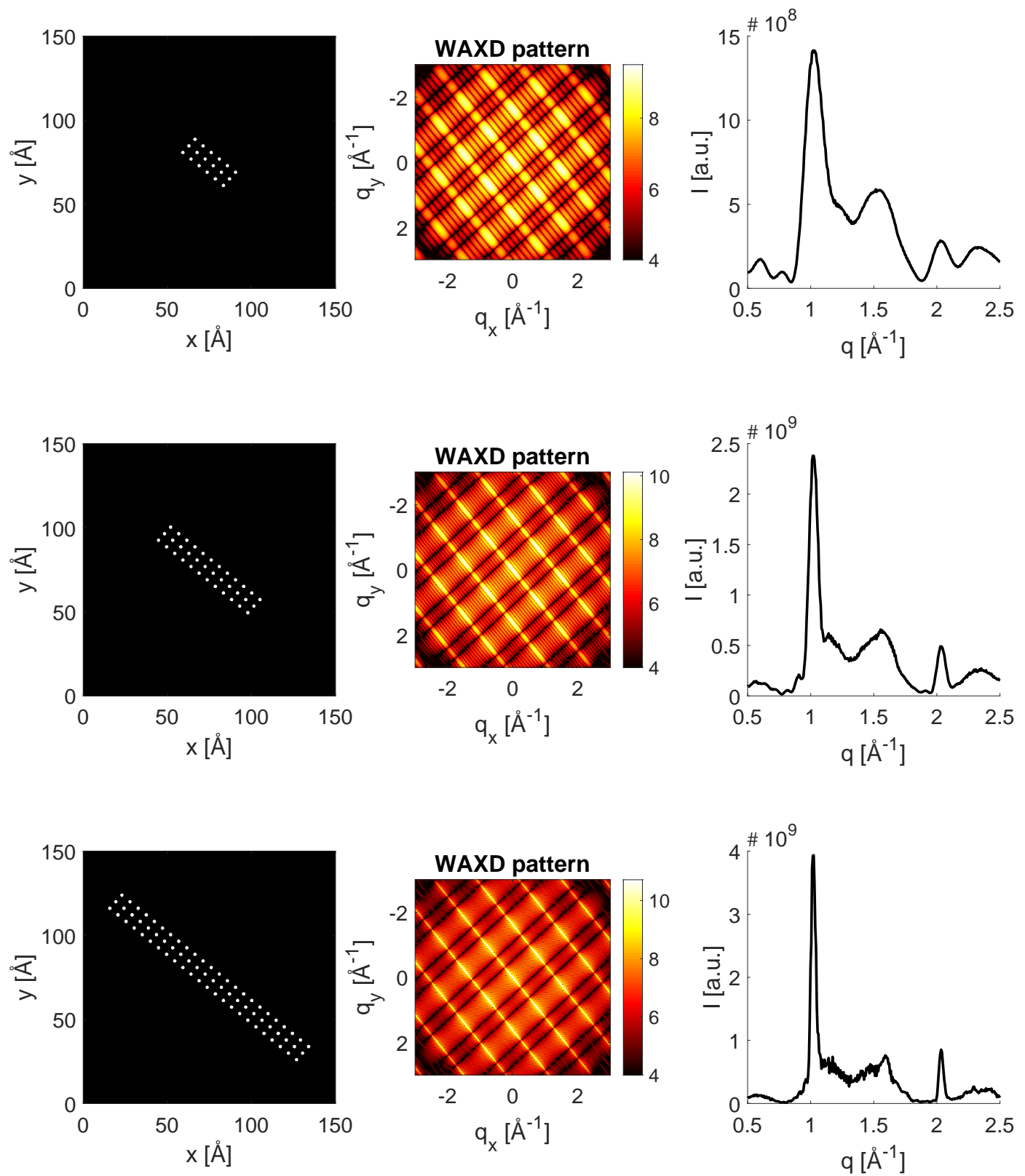
Model: R2+

Aggregation direction: (110)

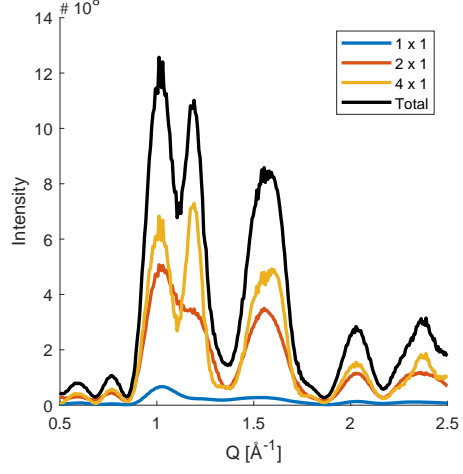

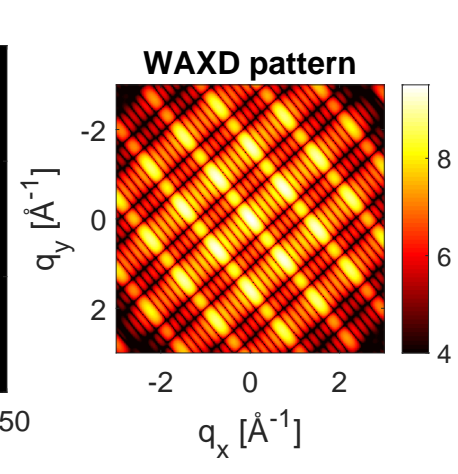

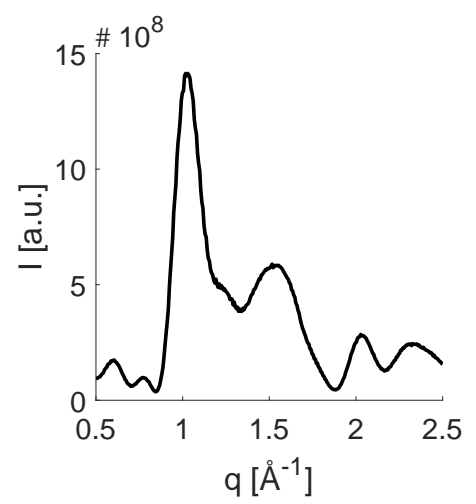

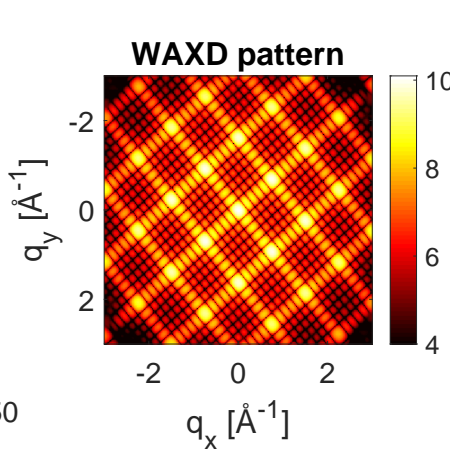

$q_{x}\left[\AA^{-1}\right]$
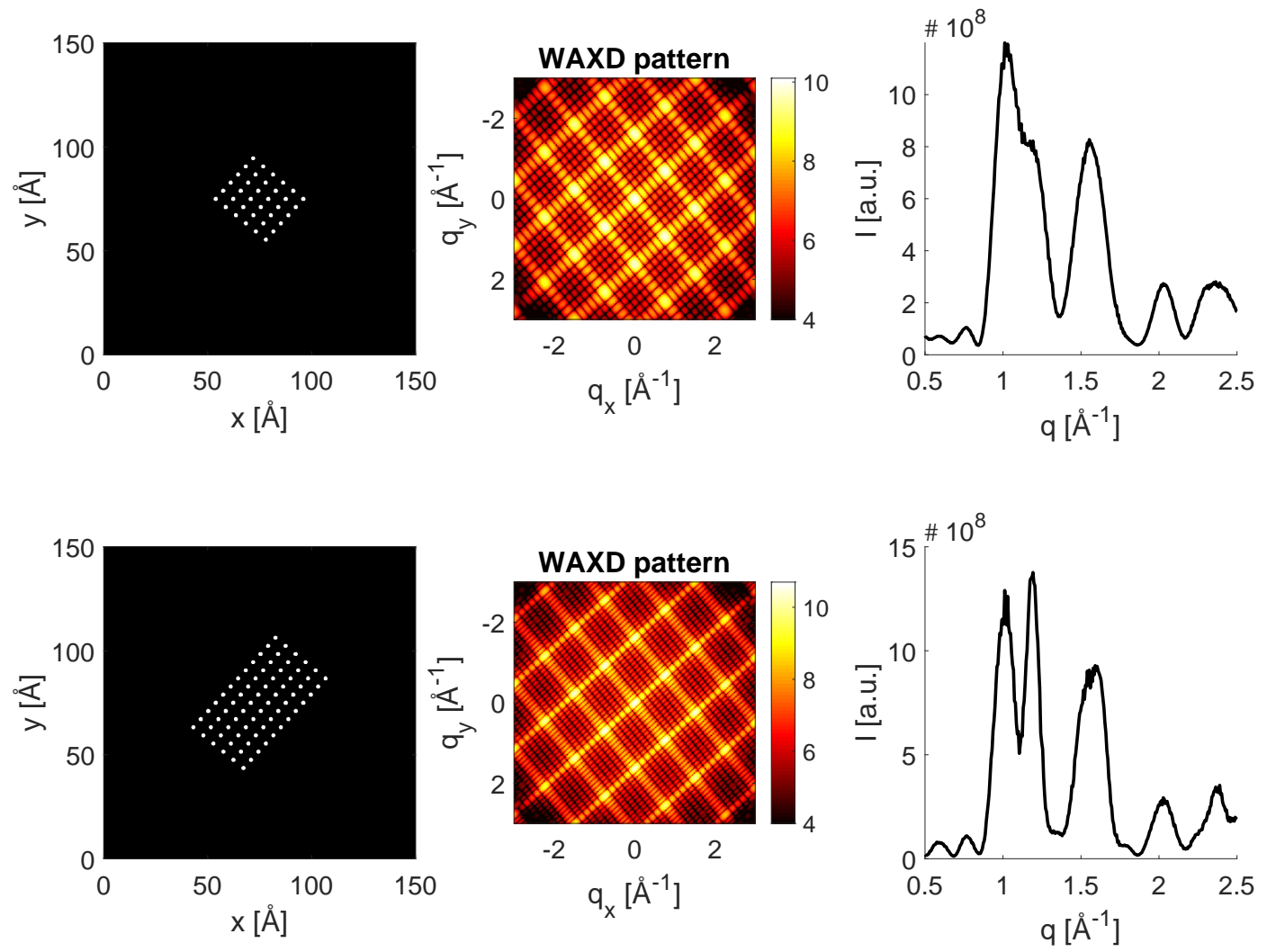
Model: R2-

Aggregation direction: (1-10)

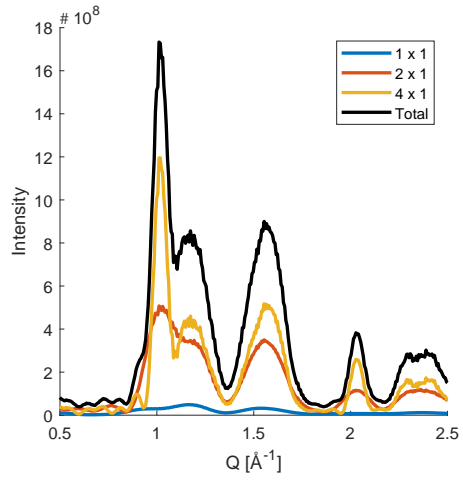

Sample \#1

Vol\% 1x1: 4.7

Vol\% $2 \times 1: 42.3$

Vol\% $4 \times 1: 53.0$
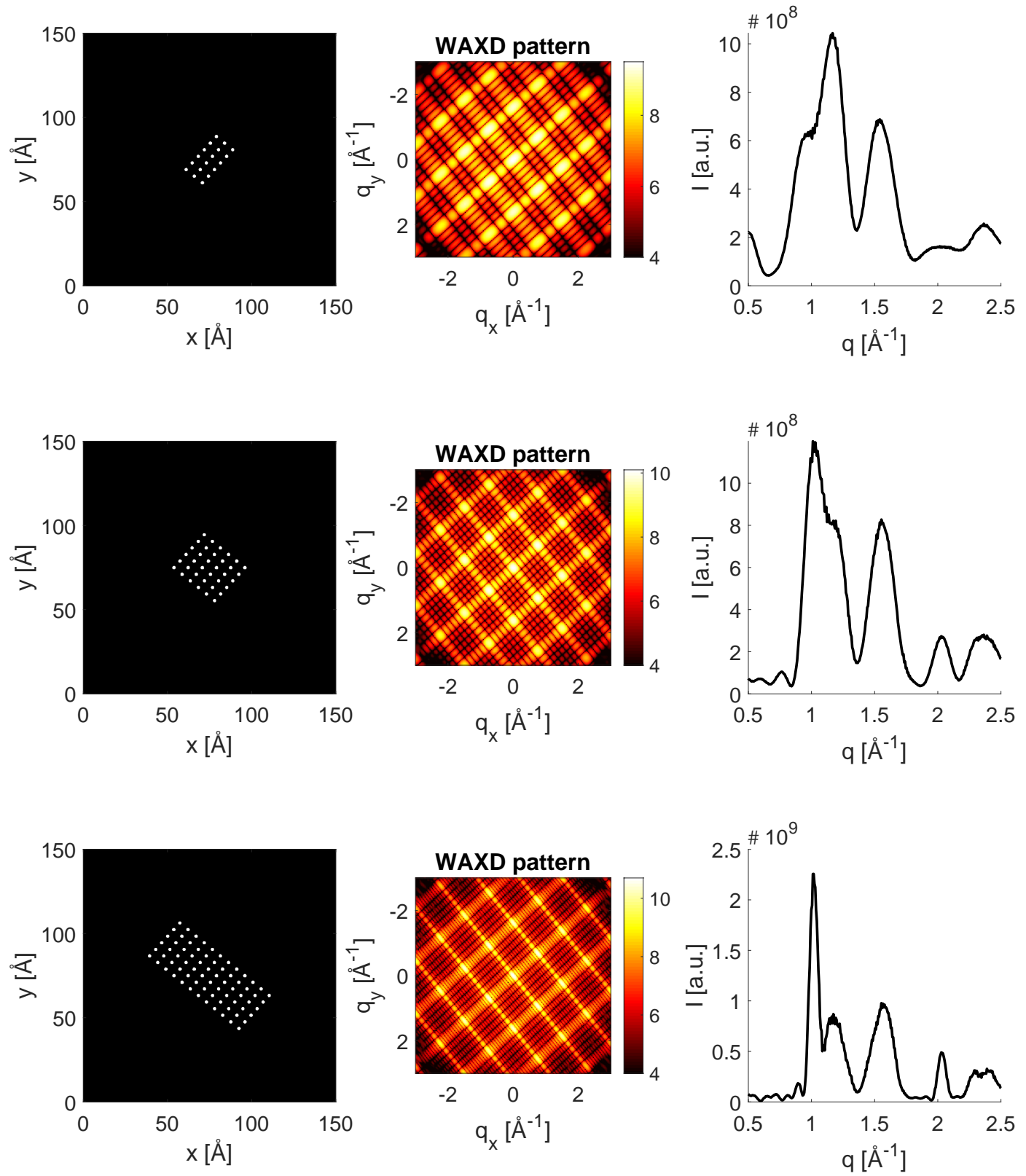
Model: R2-

Aggregation direction: (110)

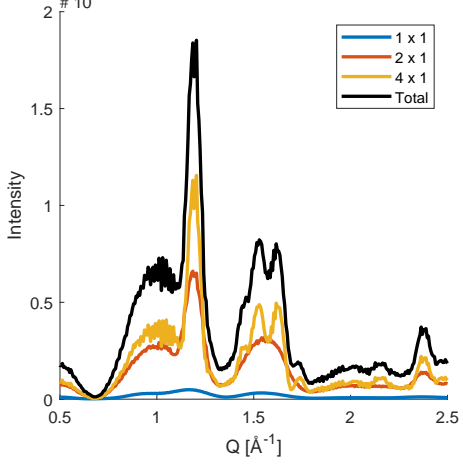

Sample \#1

Vol\% 1x1: 4.7

Vol\% 2x1: 42.3

Vol\% $4 \times 1: 53.0$
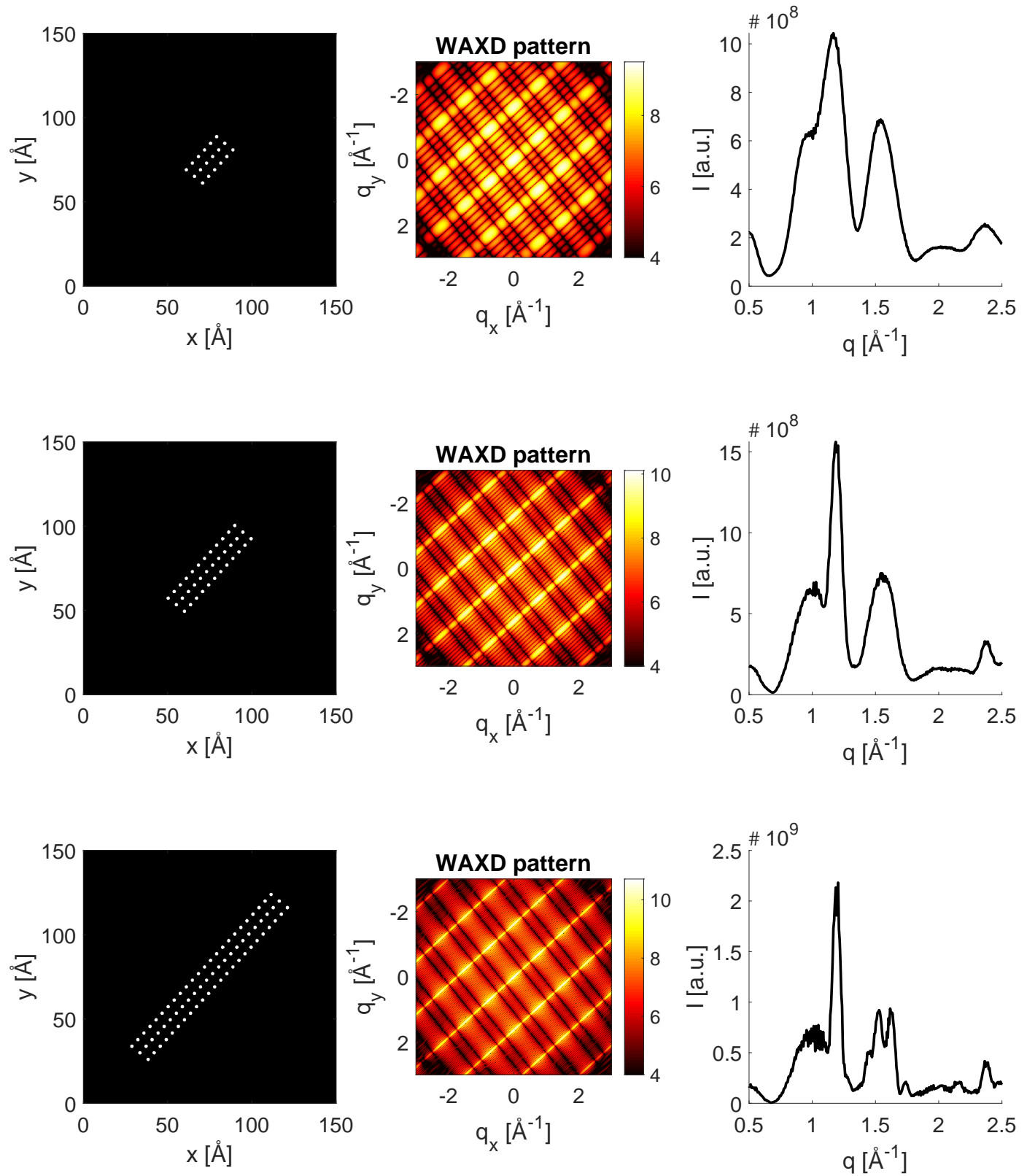
Model: Rh1+

Aggregation direction: (110)

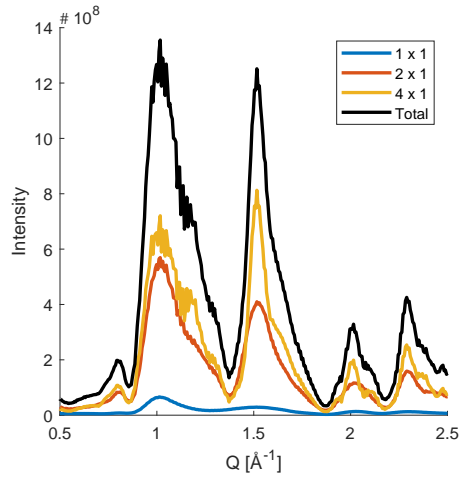

Sample \#1

Vol\% 1x1: 4.7

Vol\% $2 \times 1: 42.3$

Vol\% 4x1: 53.0
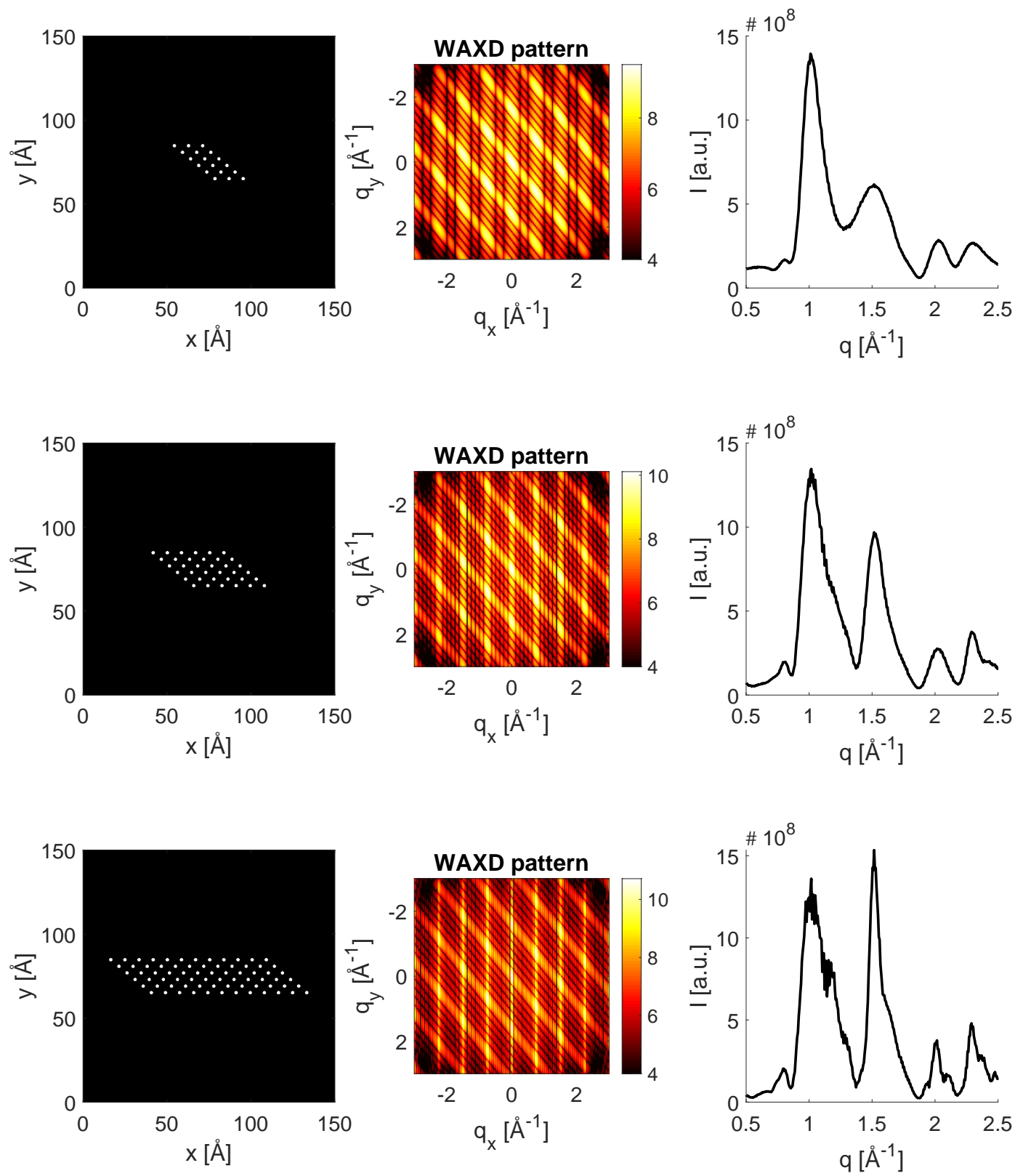
Model: Rh1+

Aggregation direction: (200)

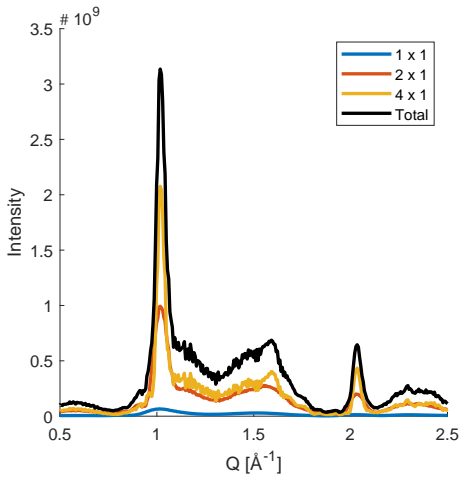

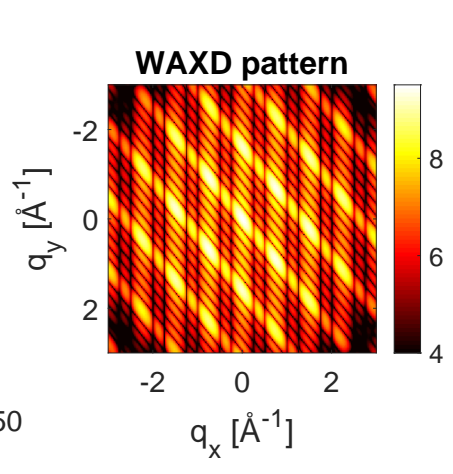

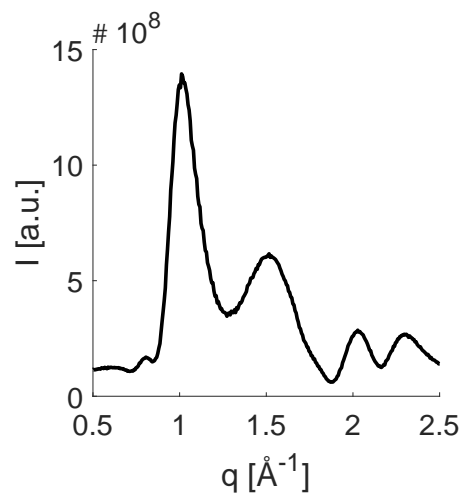

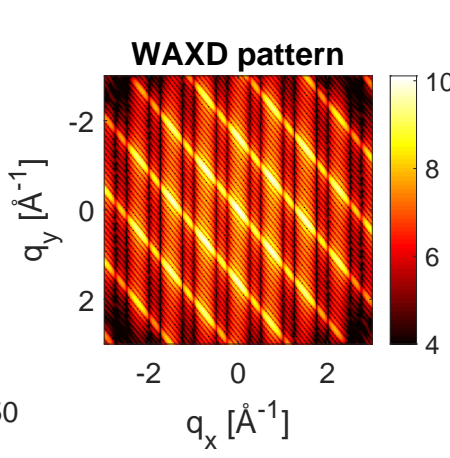

$q_{x}\left[\AA^{-1}\right]$
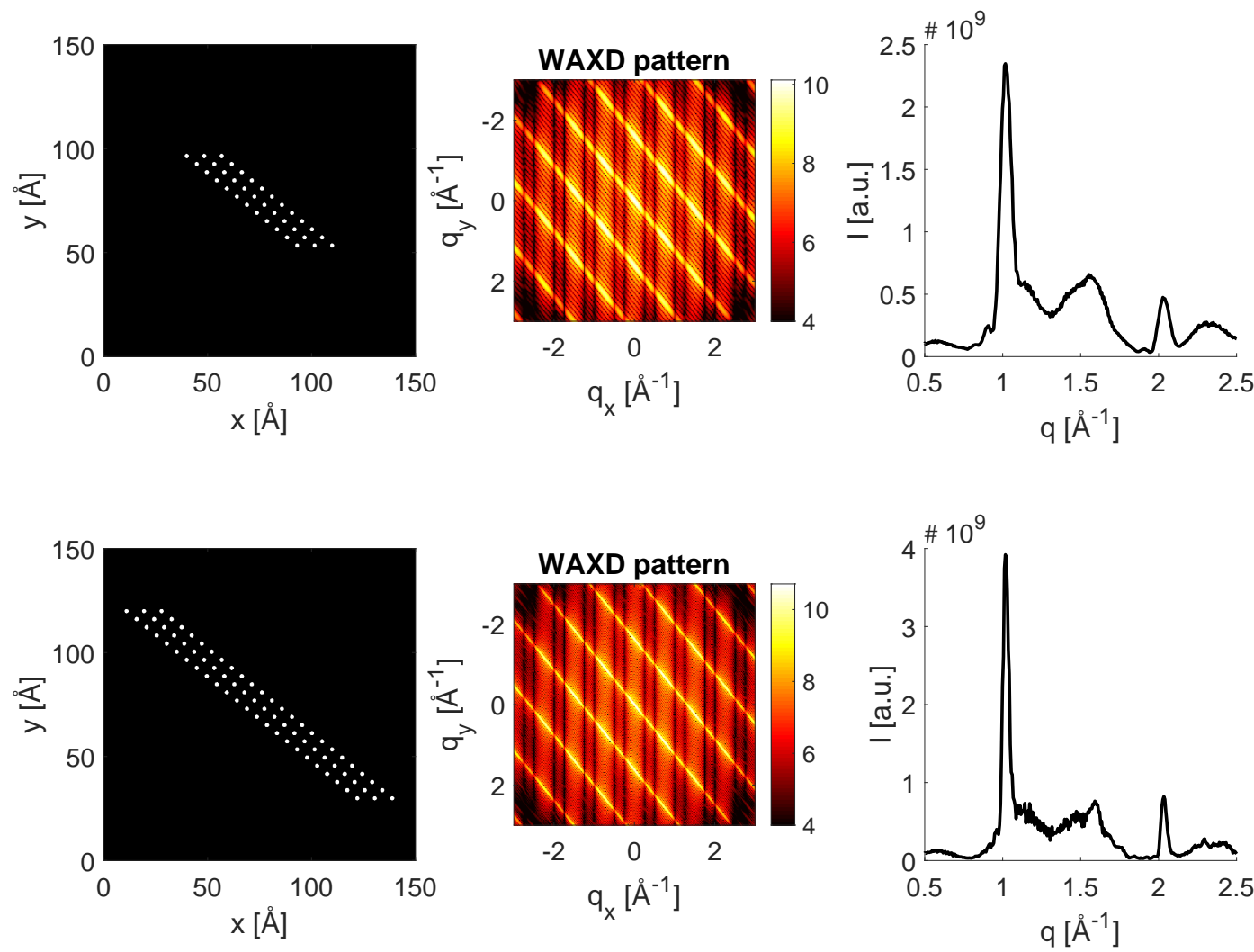
Model: Rh1-

Aggregation direction: (1-10)
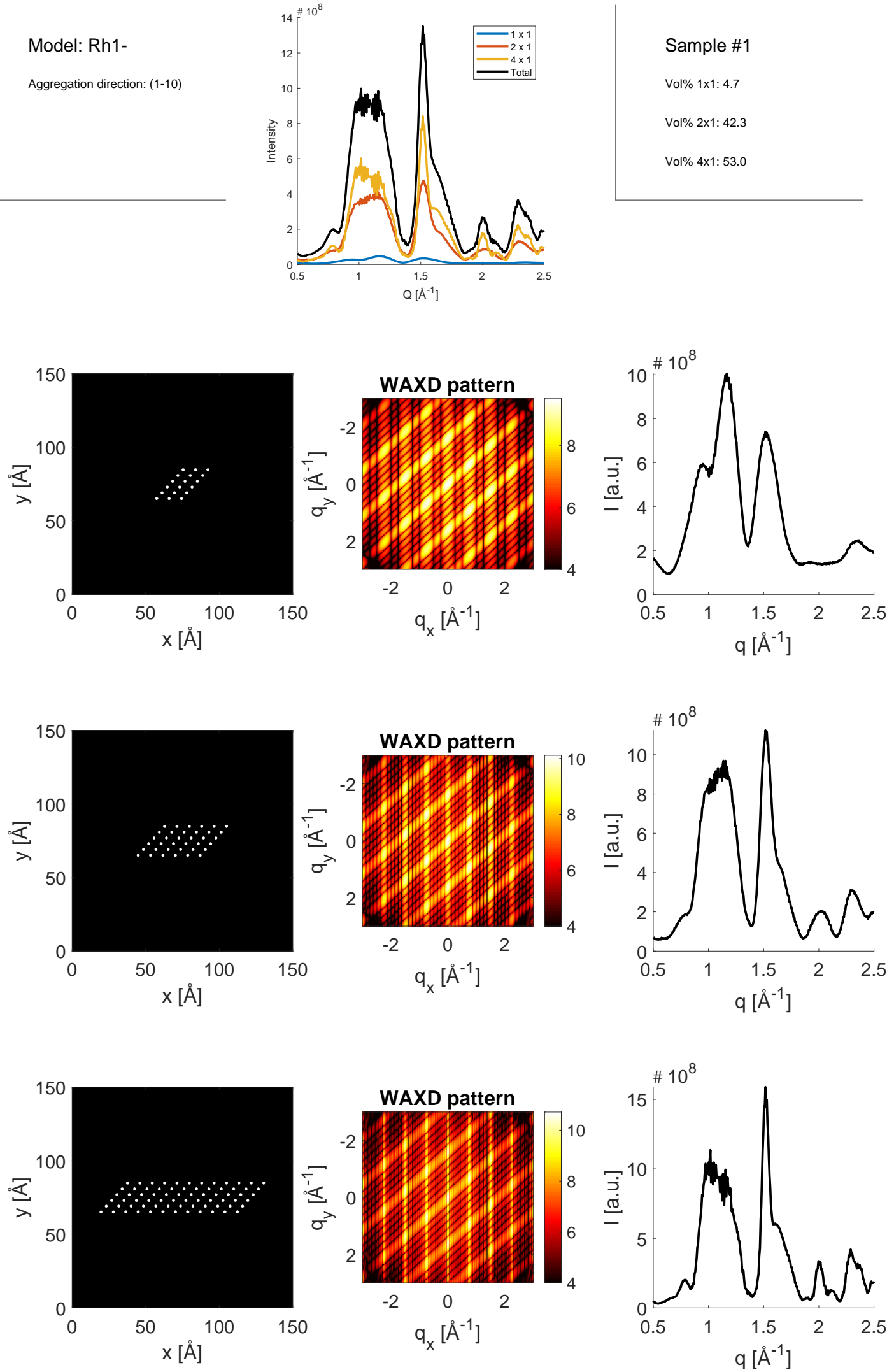
Model: Rh1-

Aggregation direction: (200)

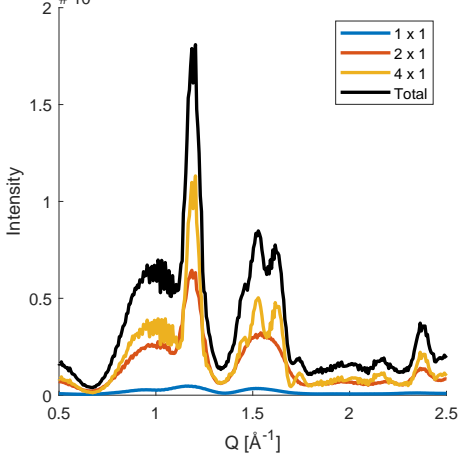

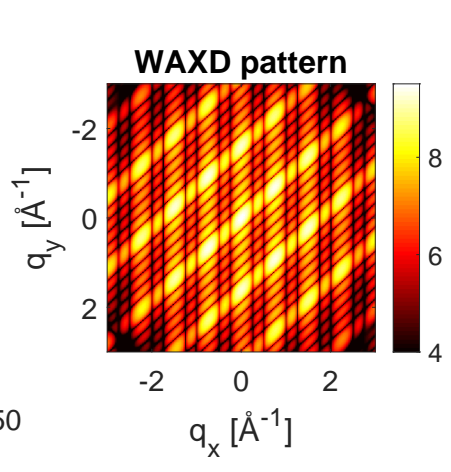
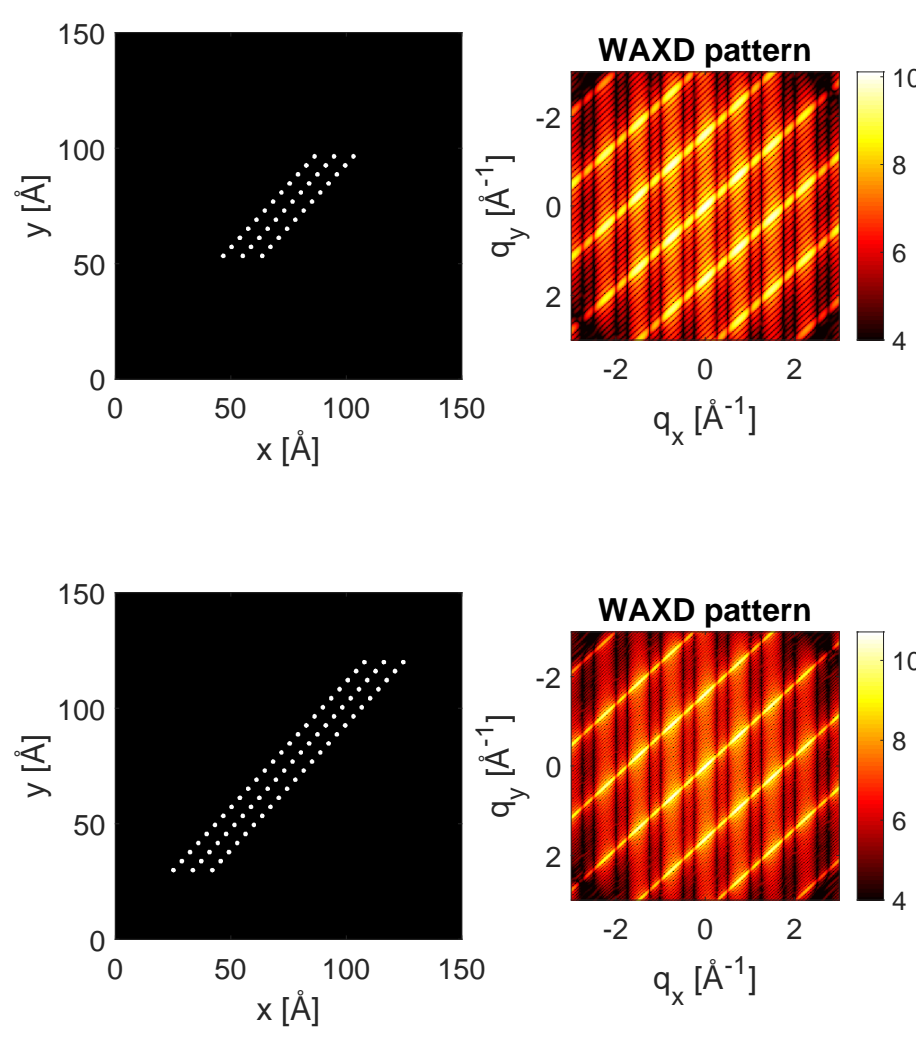

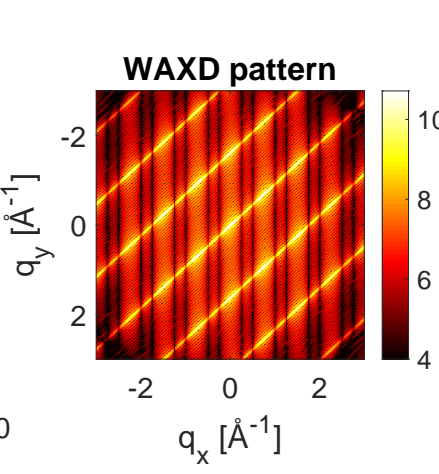

2. \#10

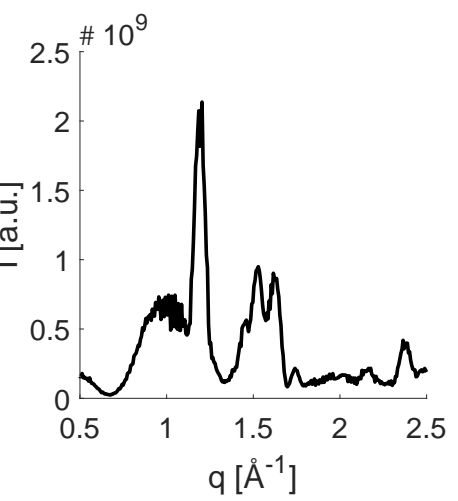

$q\left[\AA^{-1}\right]$
Sample \#1

Vol\% 1x1: 4.7

Vol\% 2x1: 42.3

Vol\% 4x1: 53.0
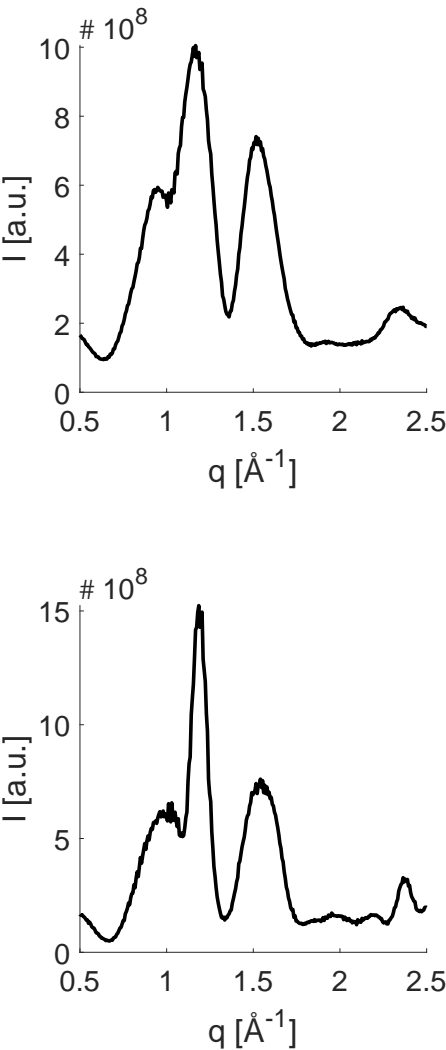
Model: Rh2

Aggregation direction: (010)
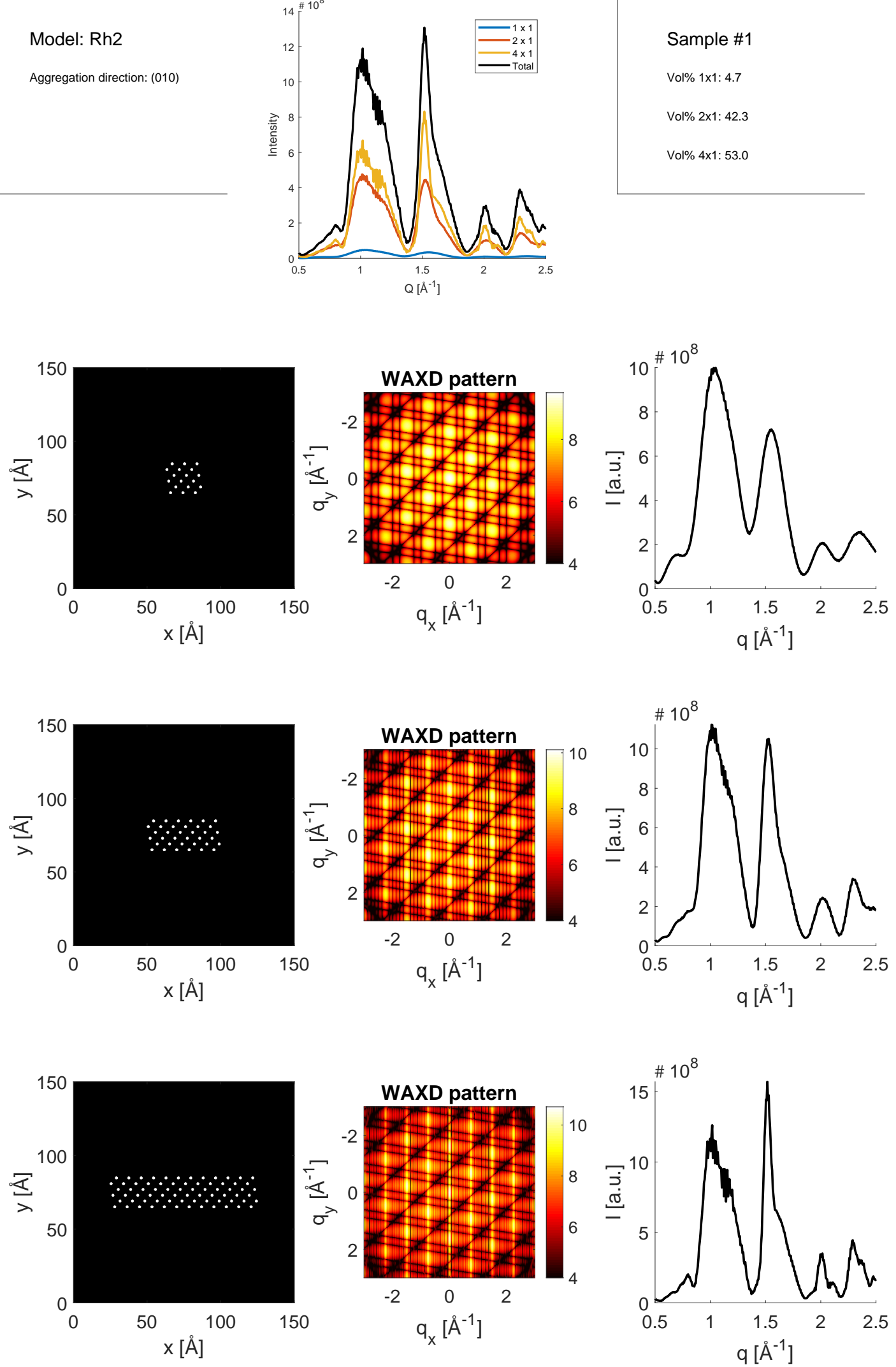
Model: Rh2

Aggregation direction: (200)

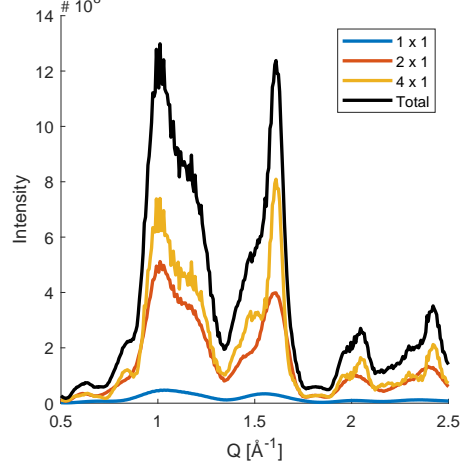

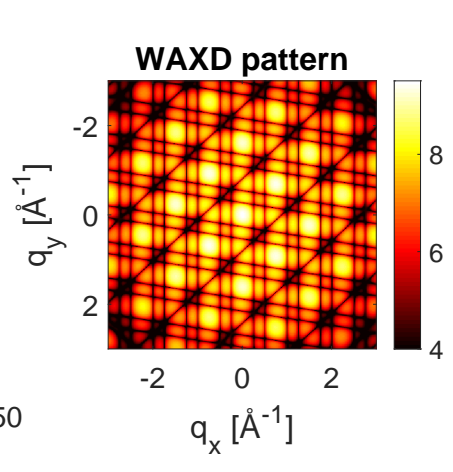
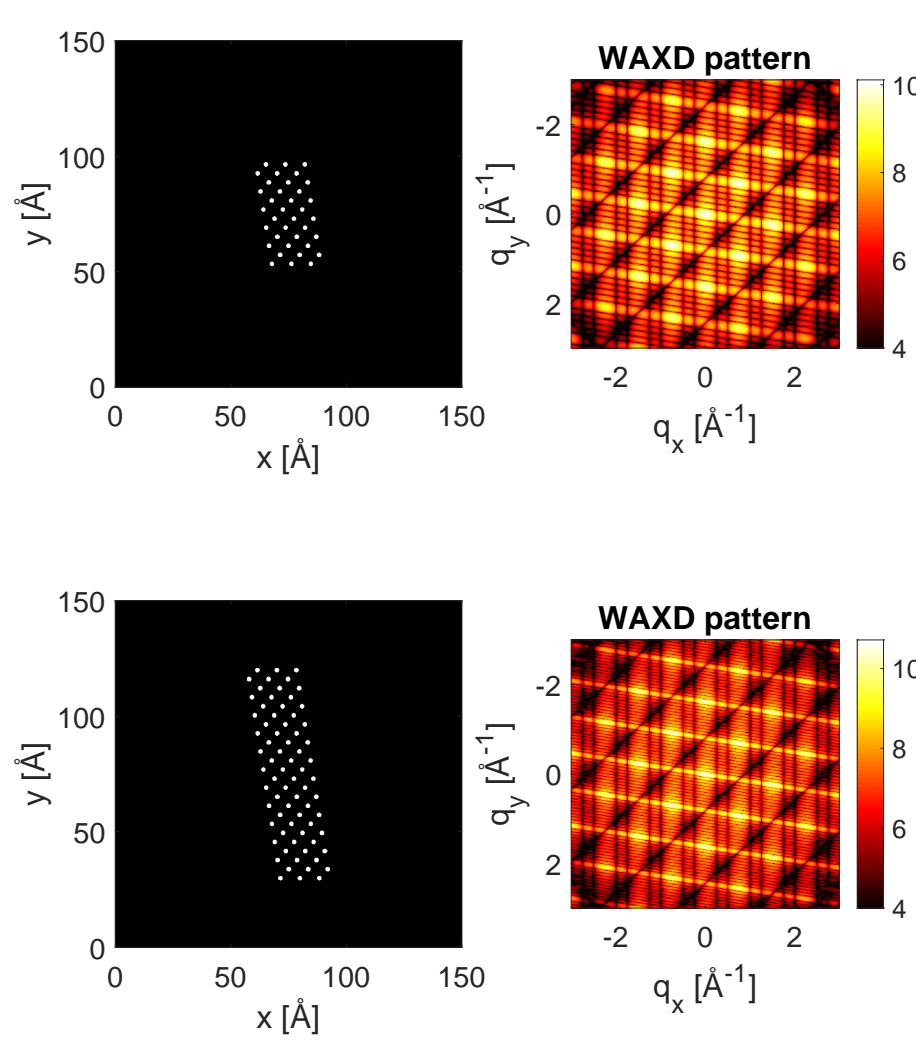
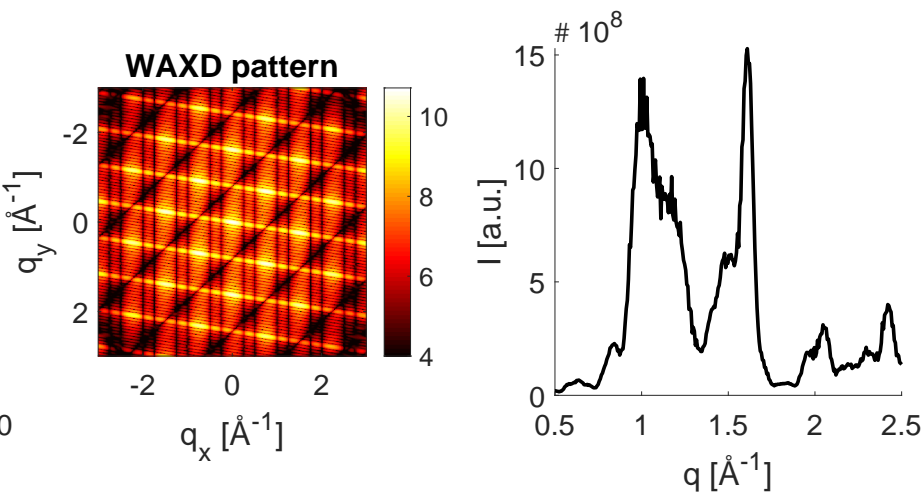

$q\left[A^{-1}\right]$
Sample \#

Vol\% 1 1 1: 4.7

Vol\% 2x1: 42.3

Vol\% 4x1: 53.0
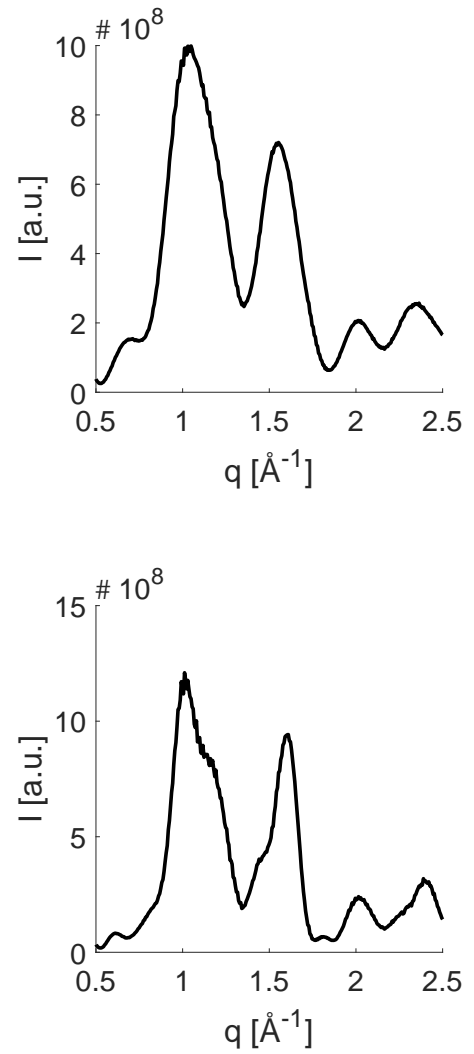

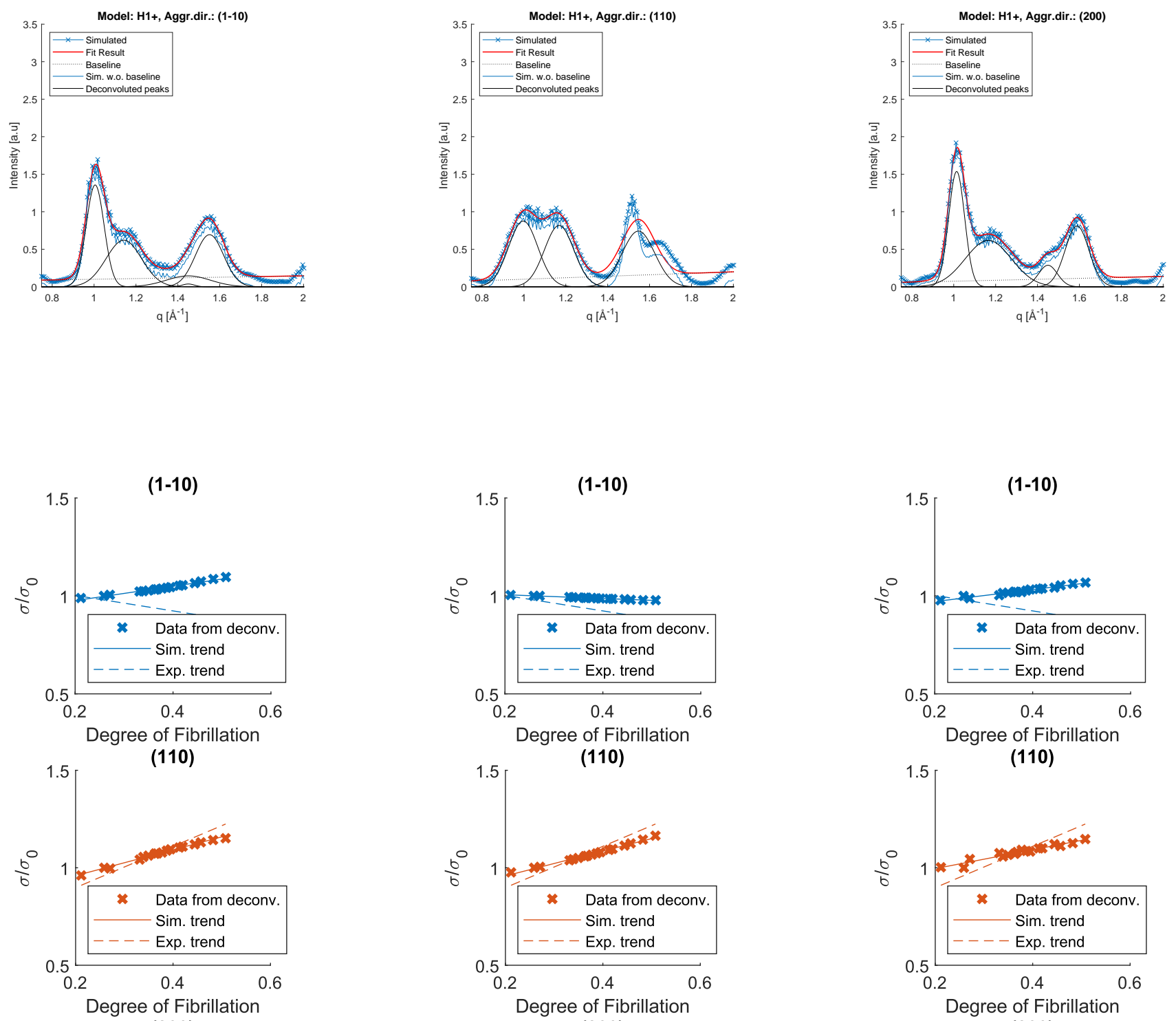

(110)

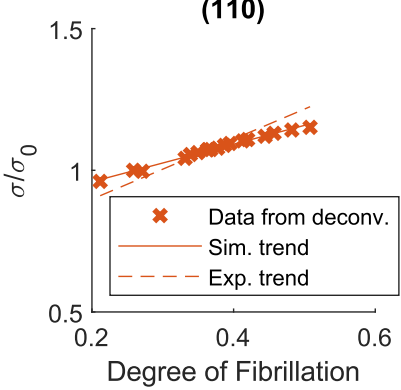

(200)
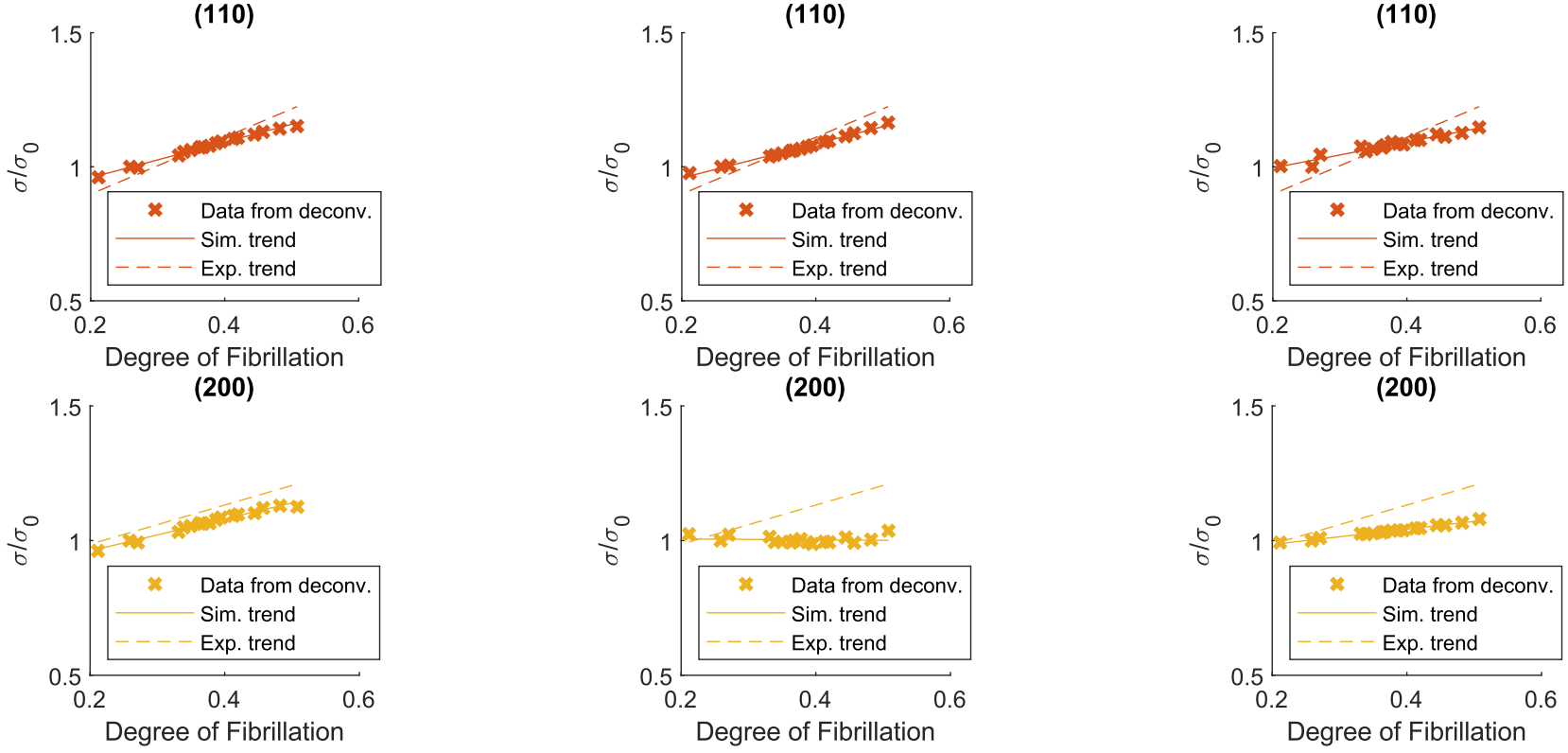

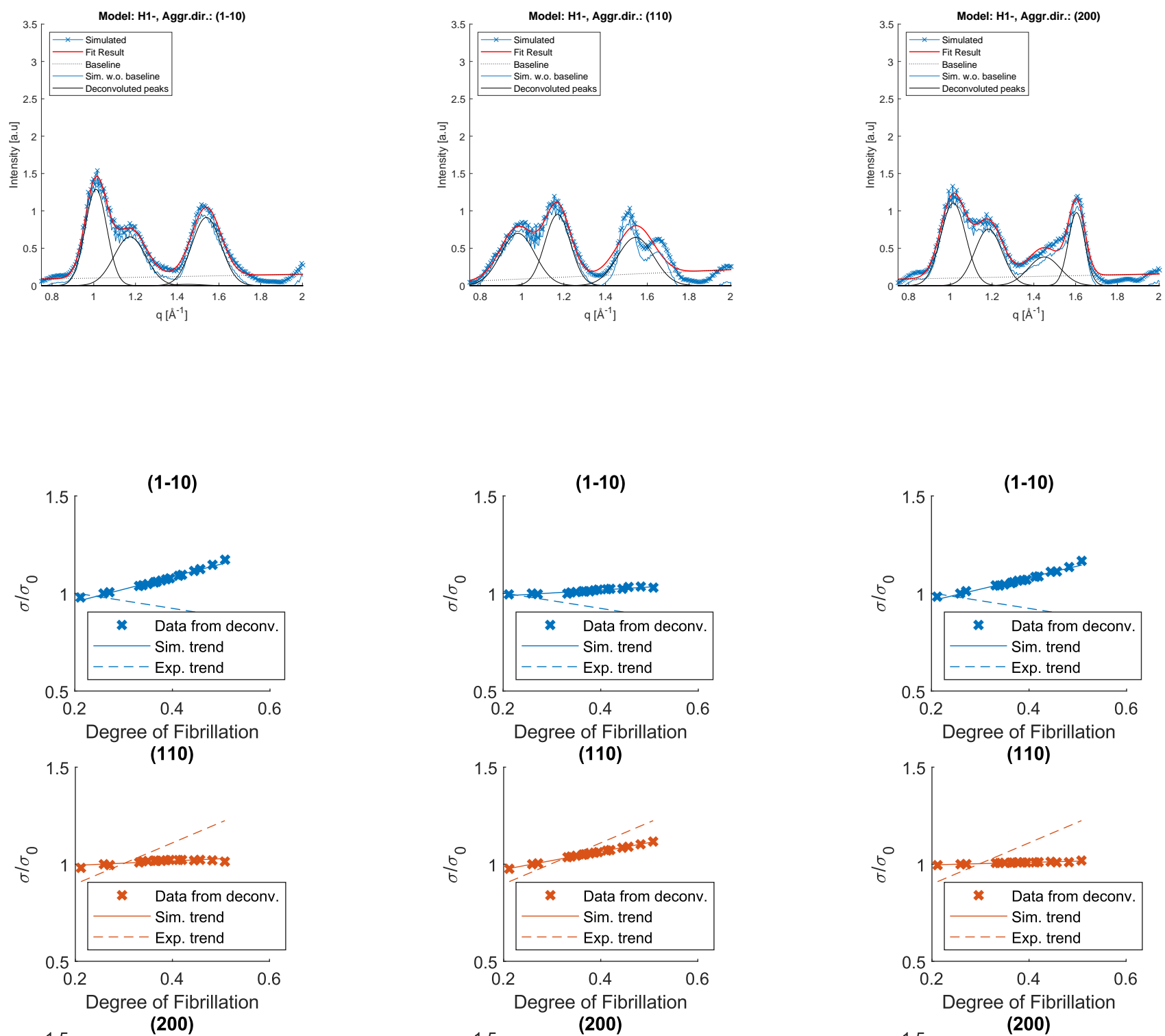

(110)
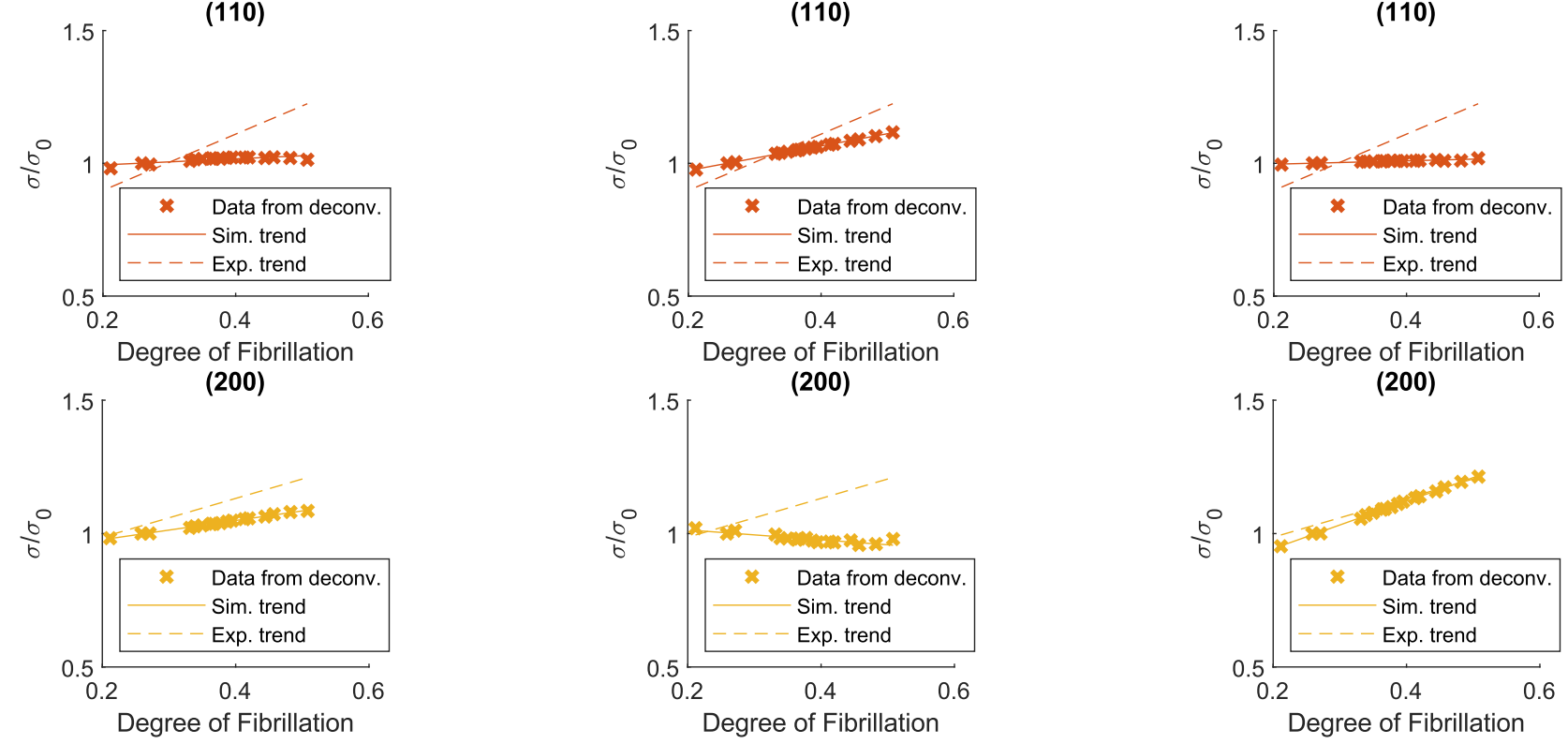

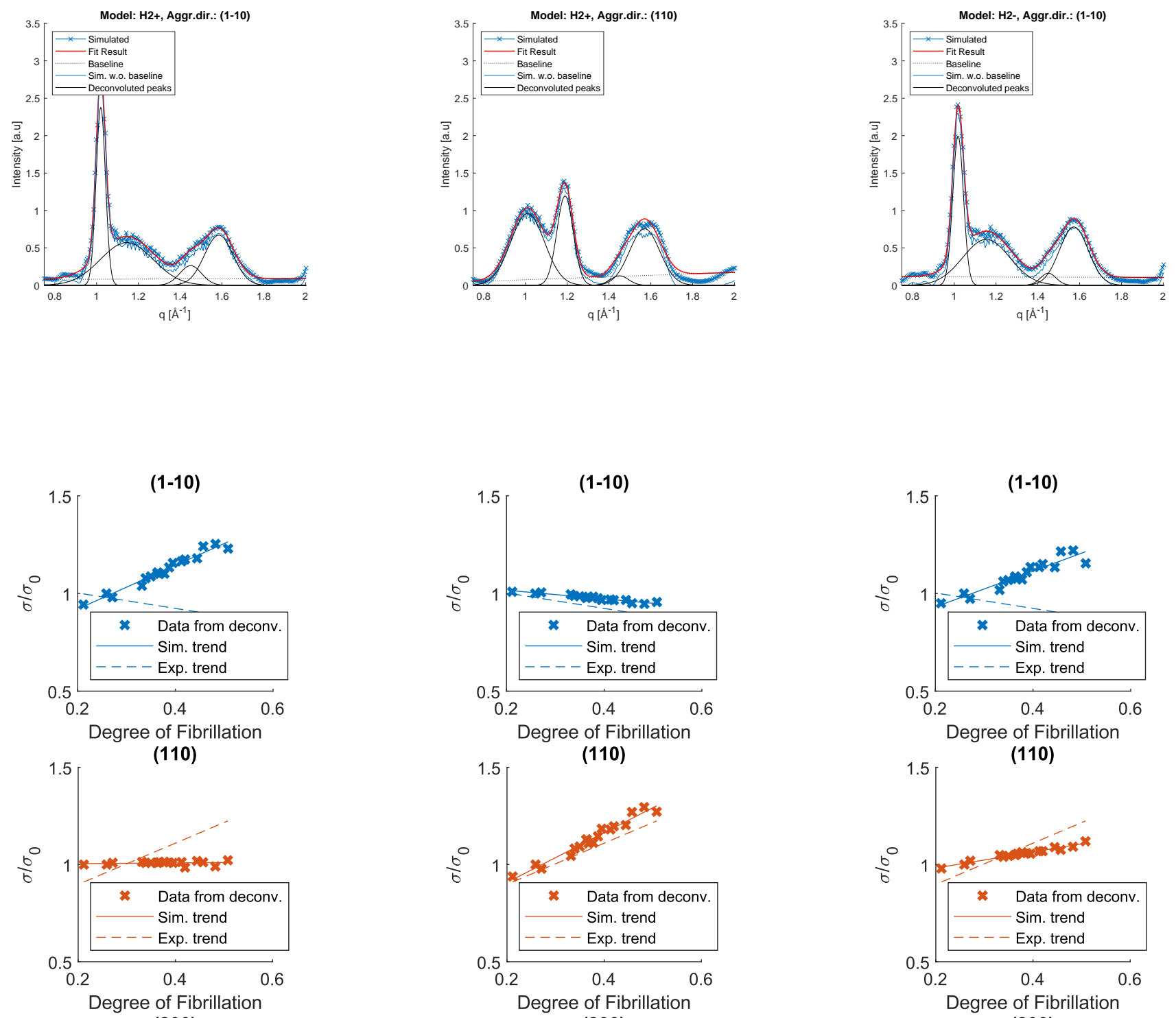

(110)
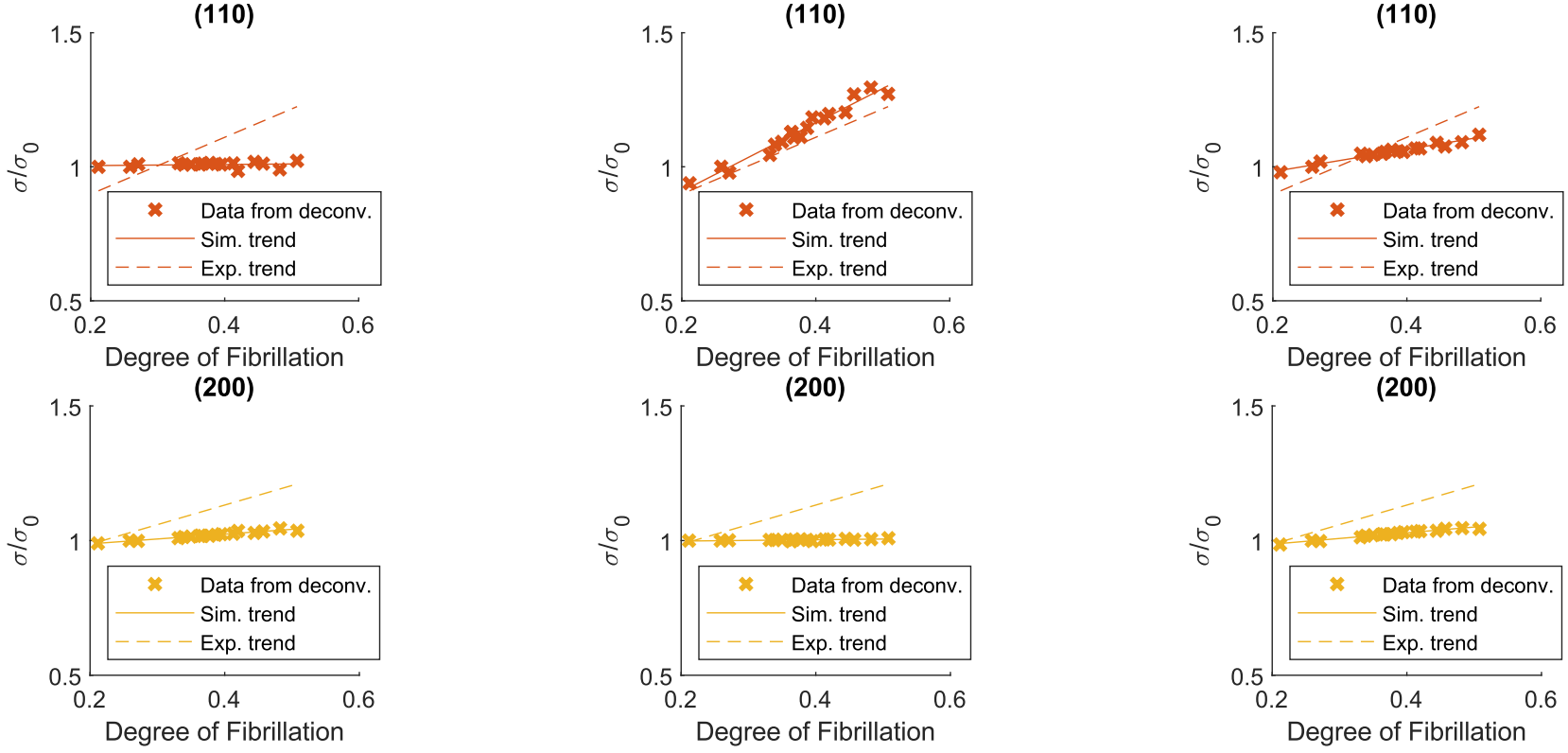

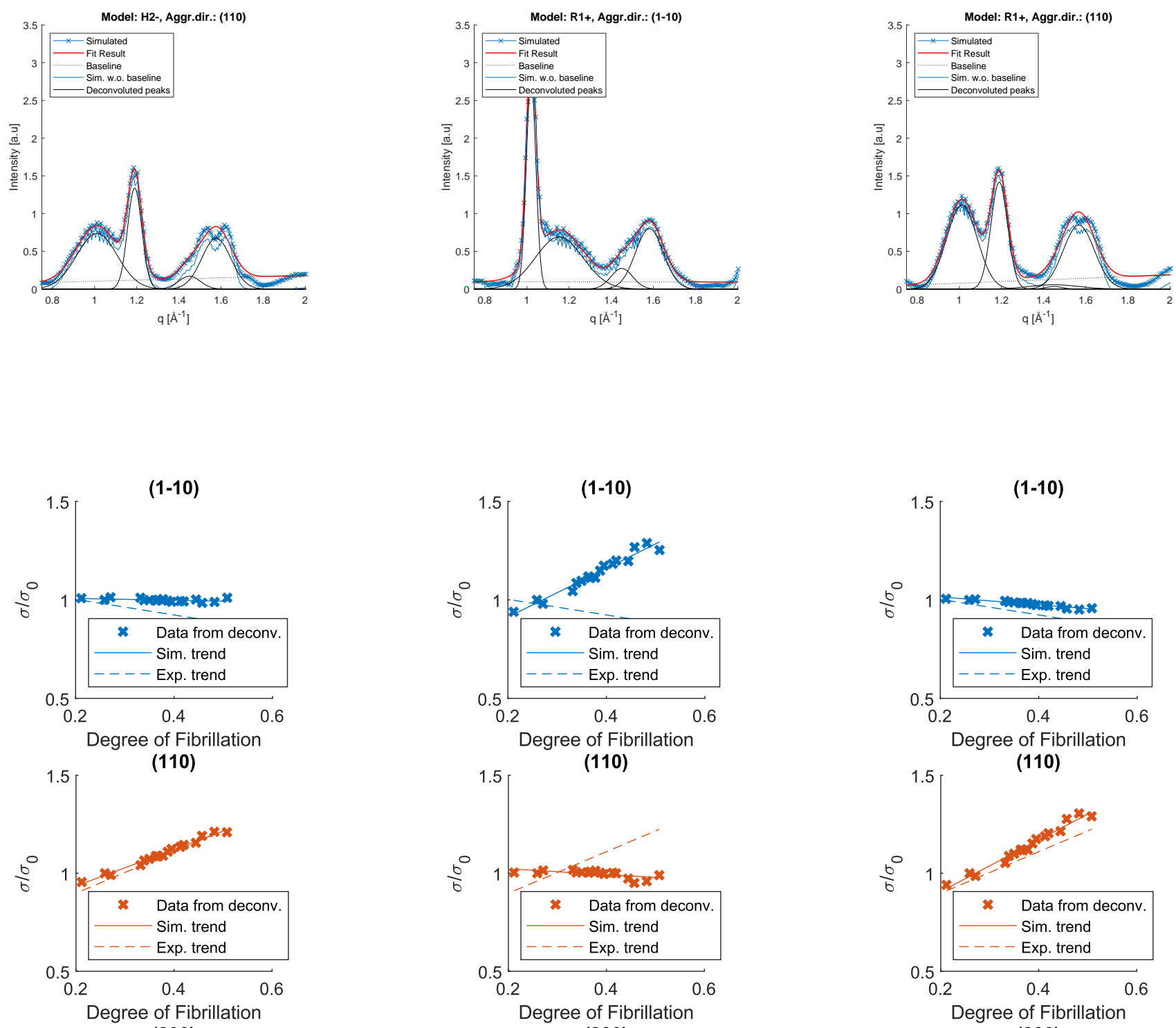

(110)

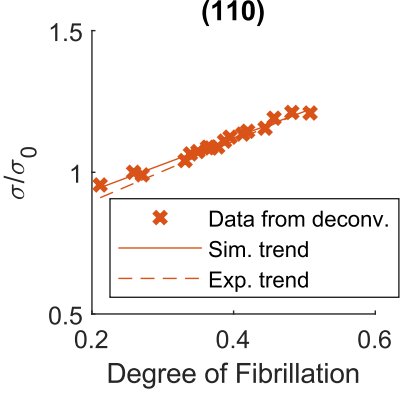

(200)
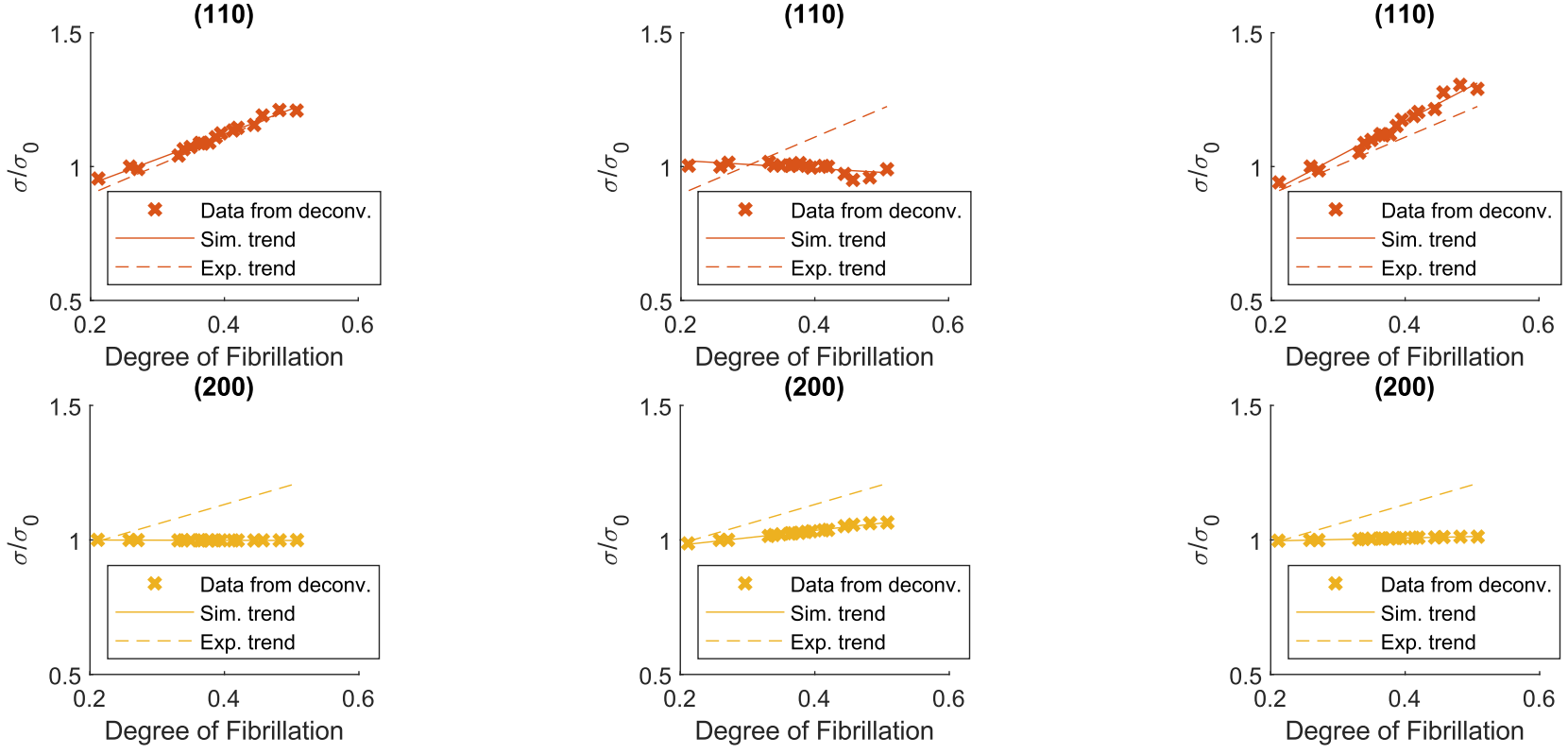

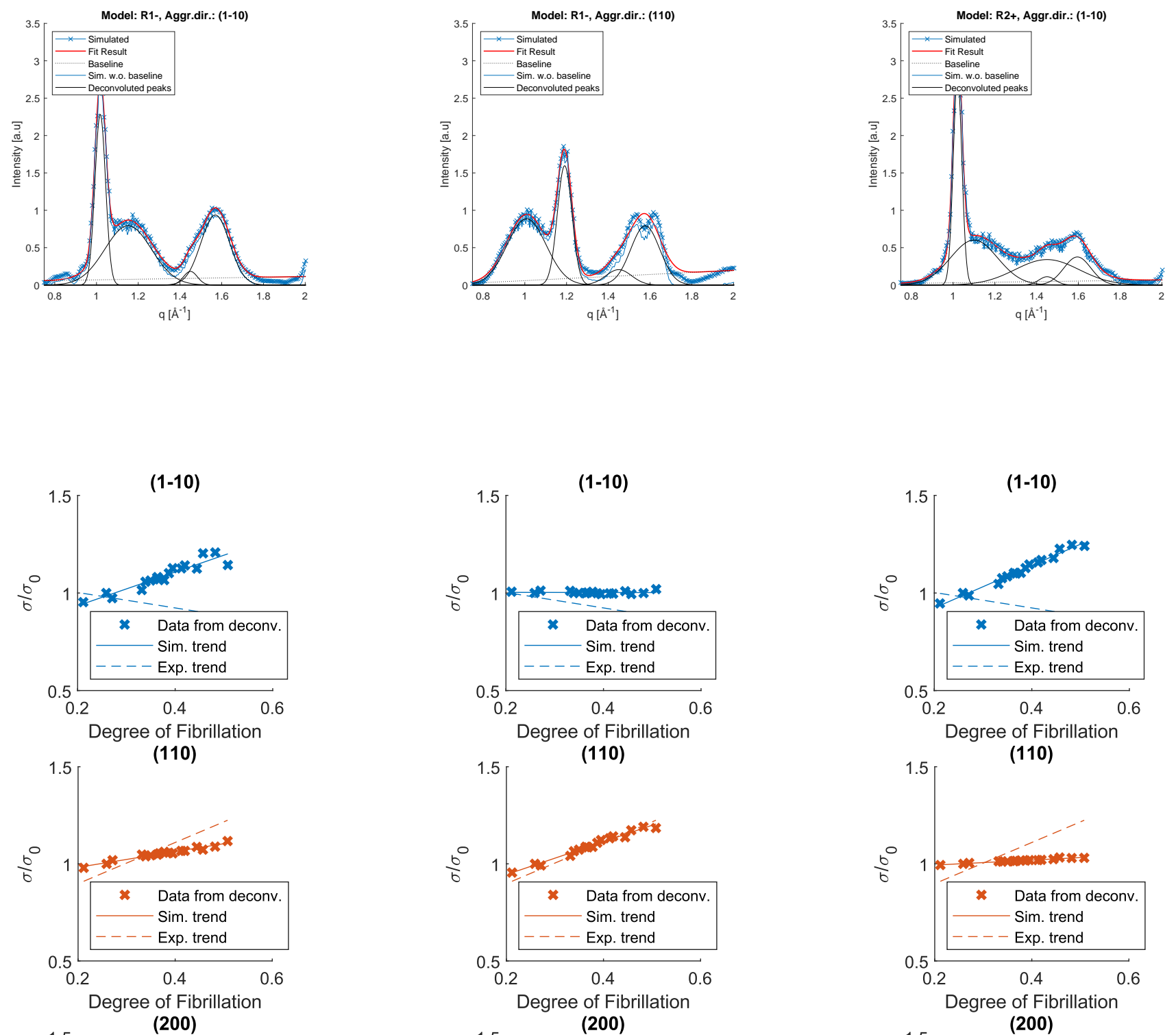

(110)
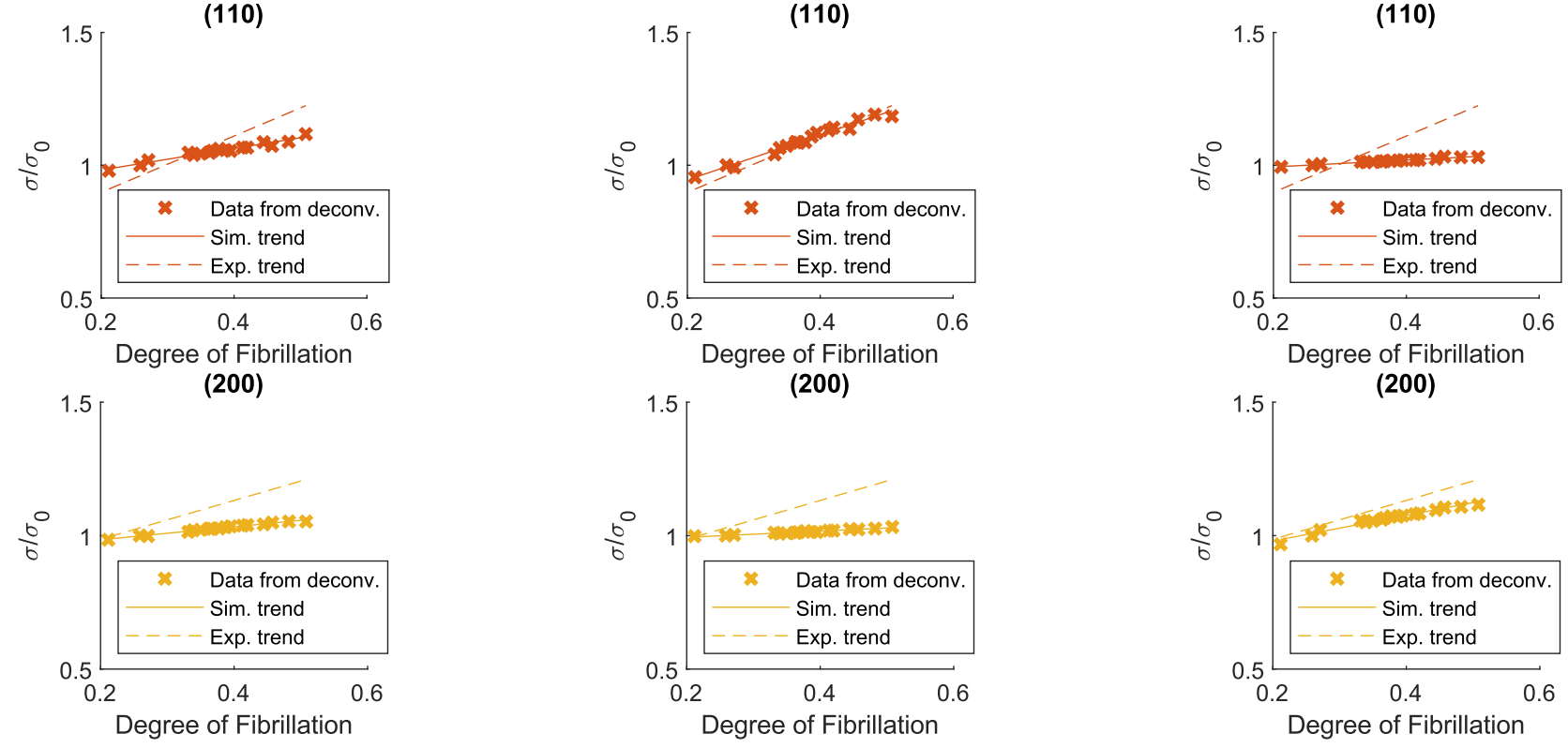

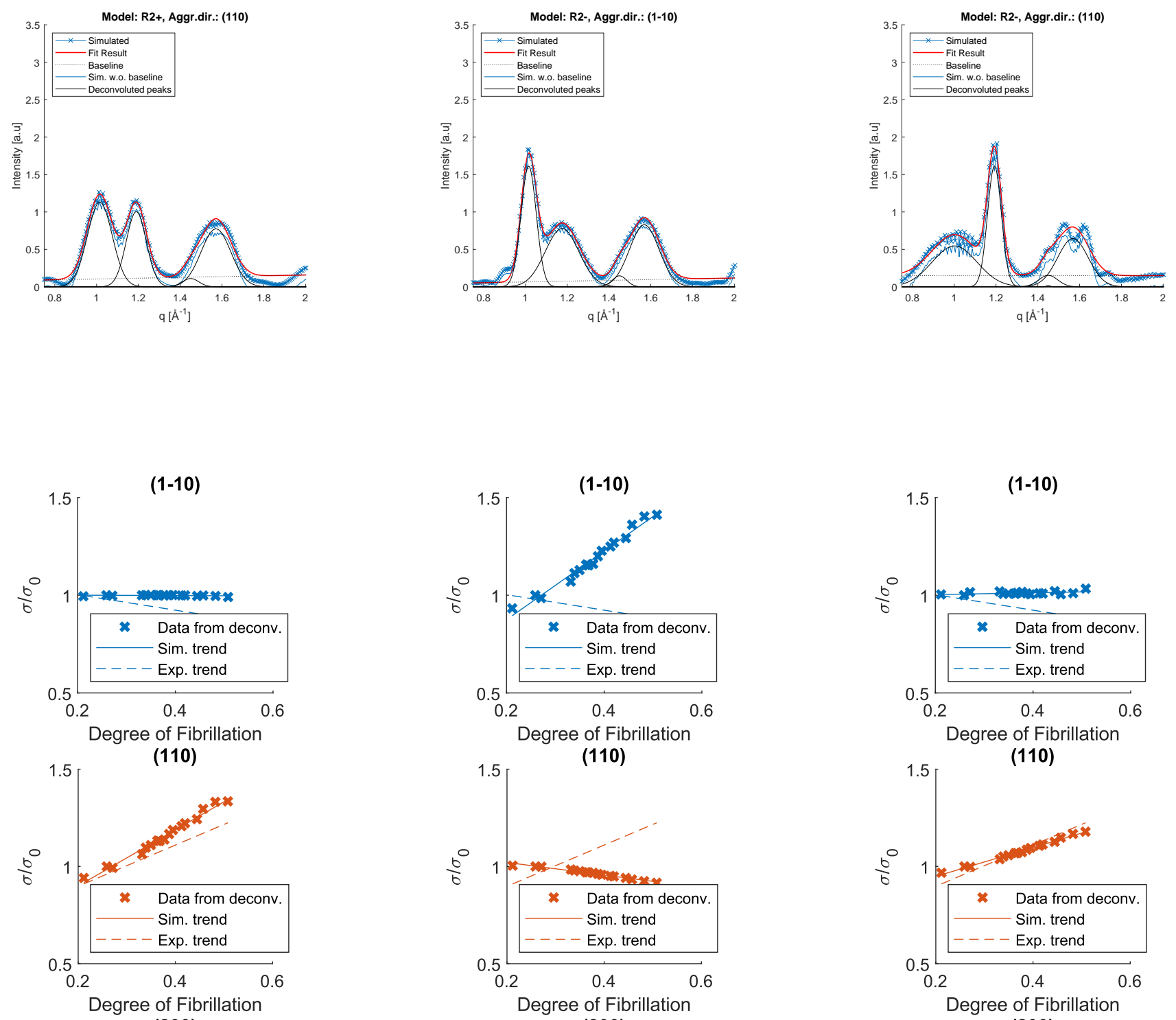

(110)

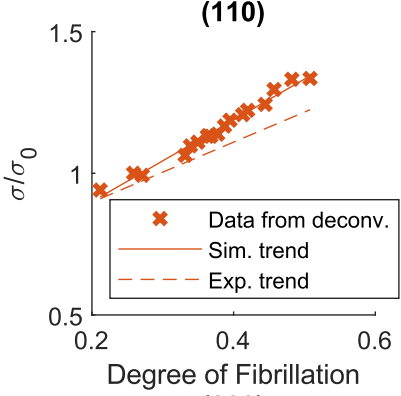

(200)
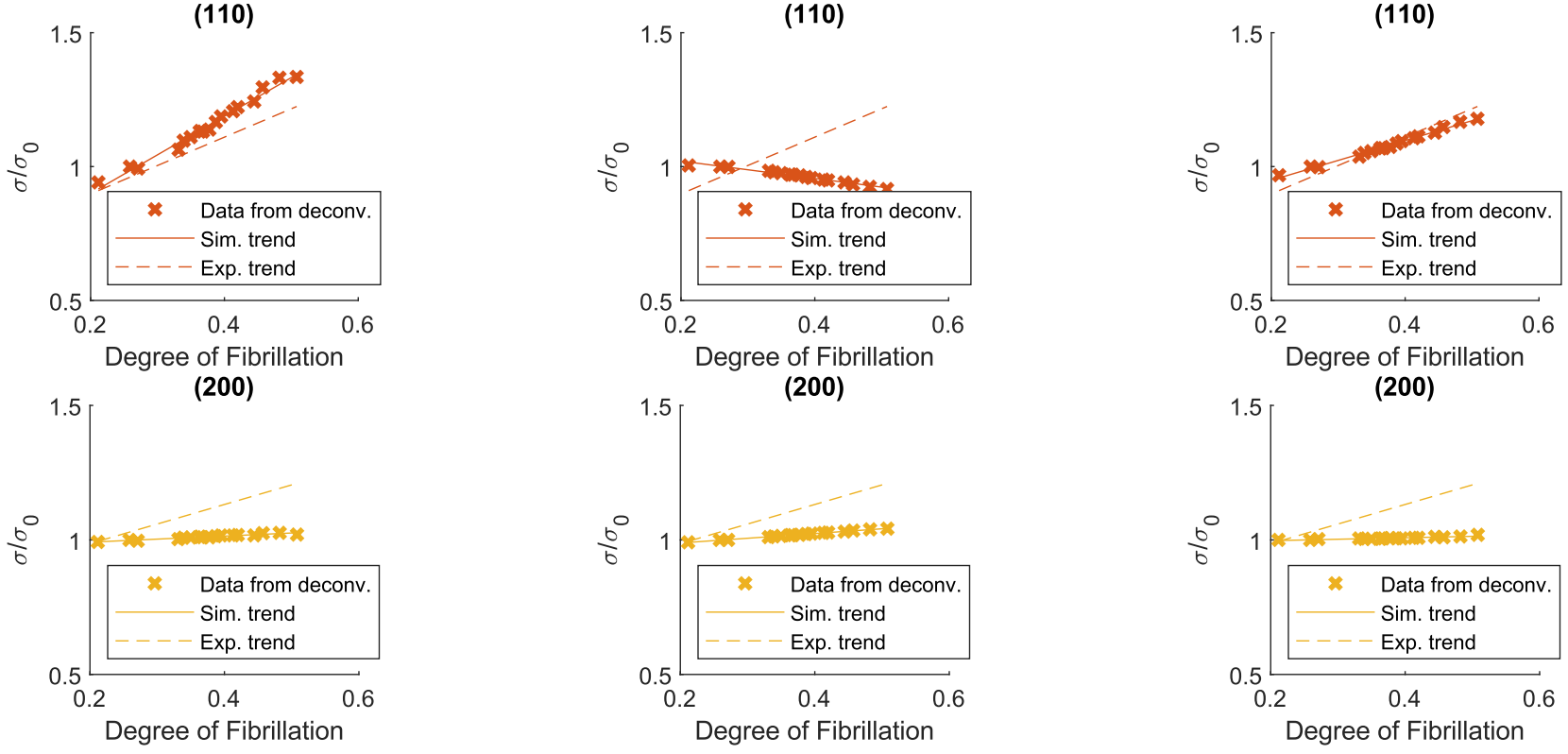

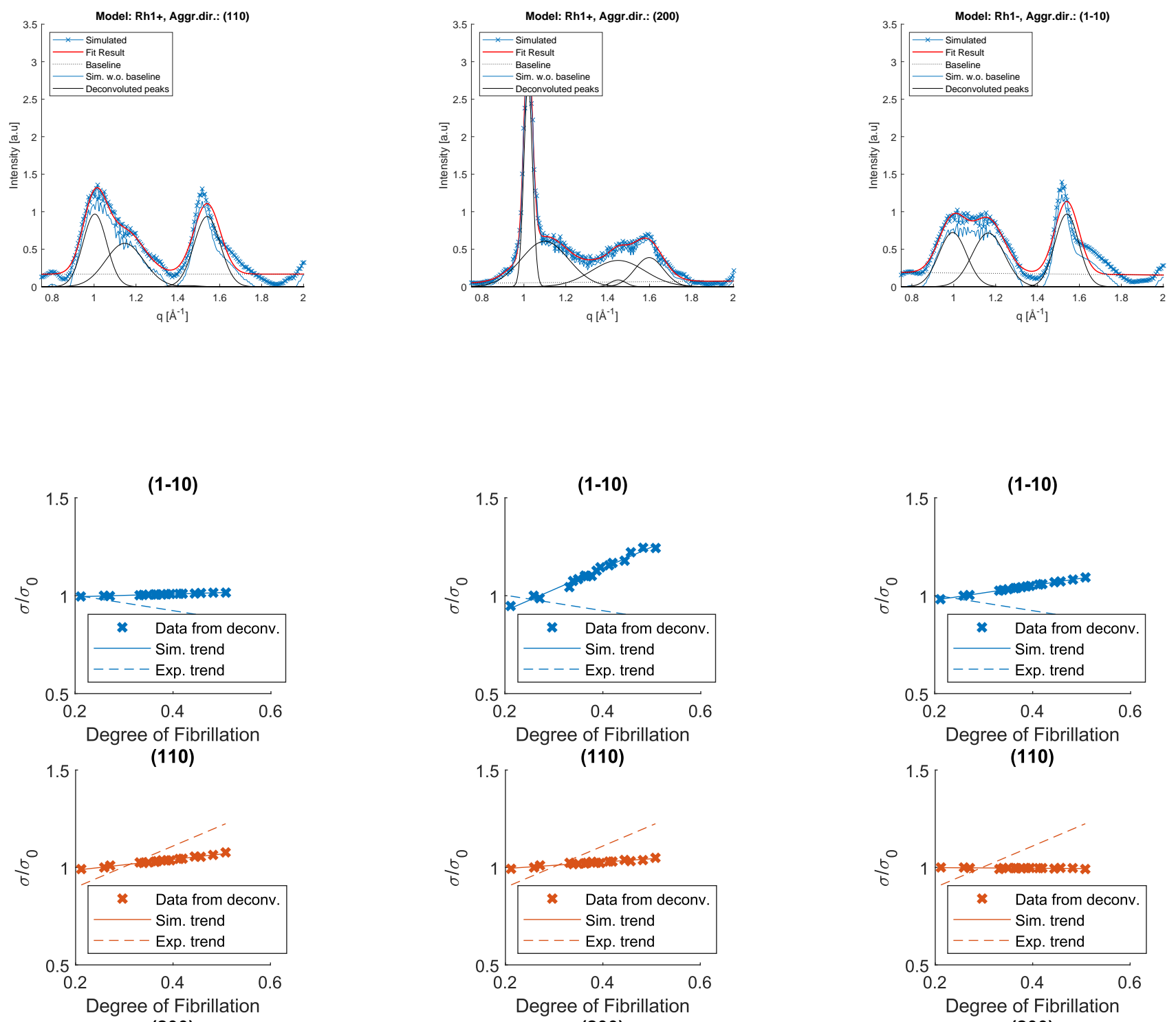

(110)
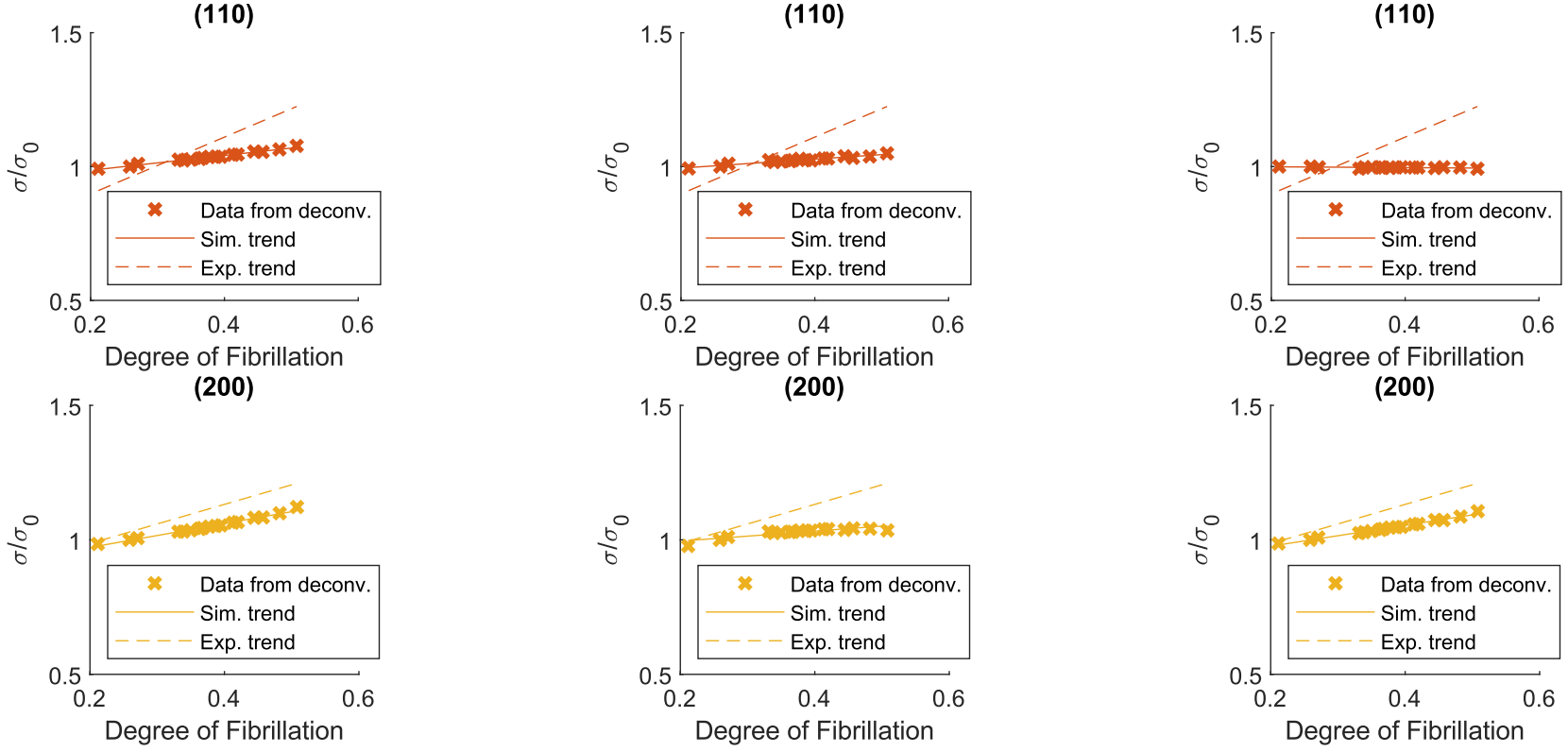

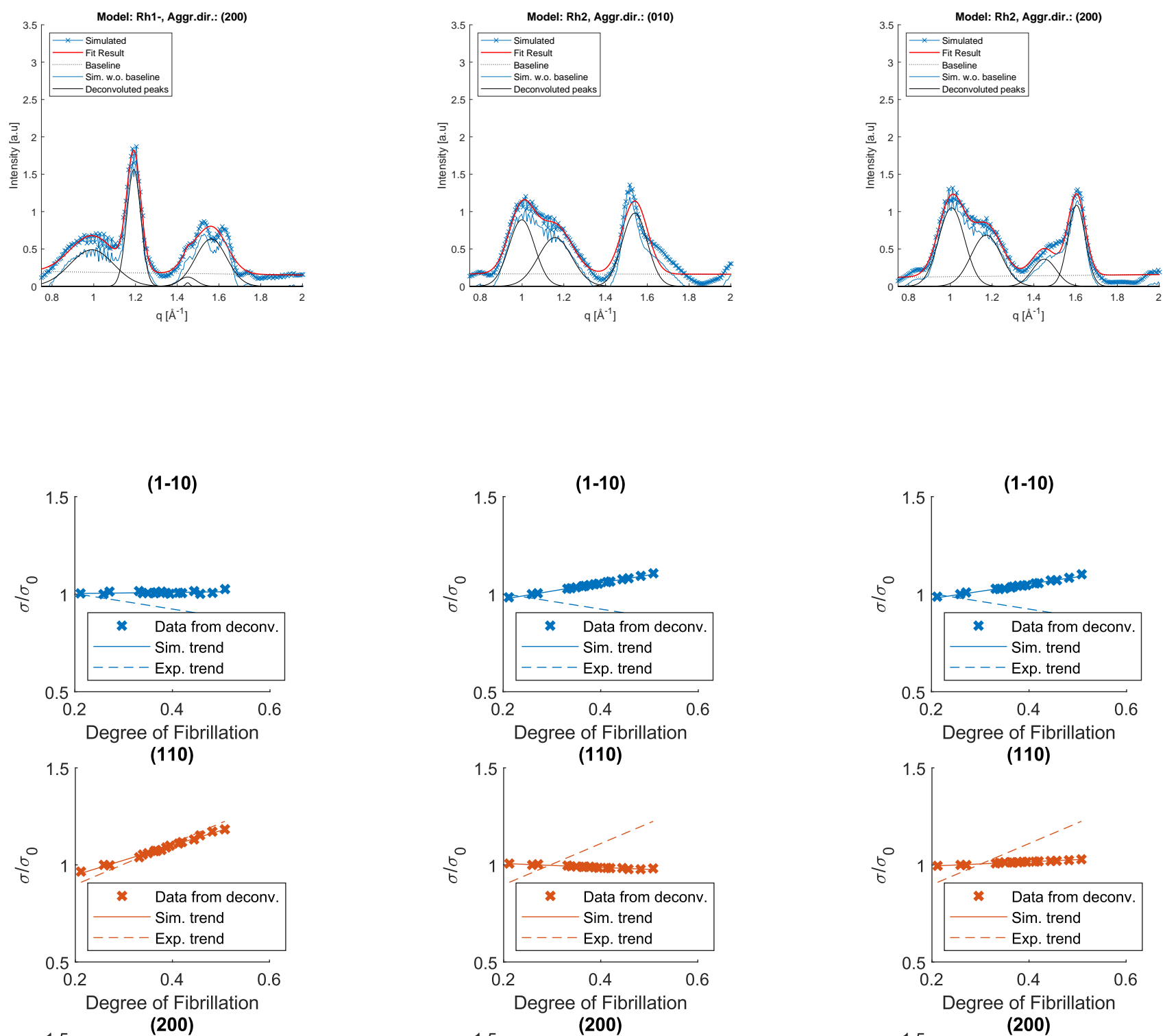

(110)
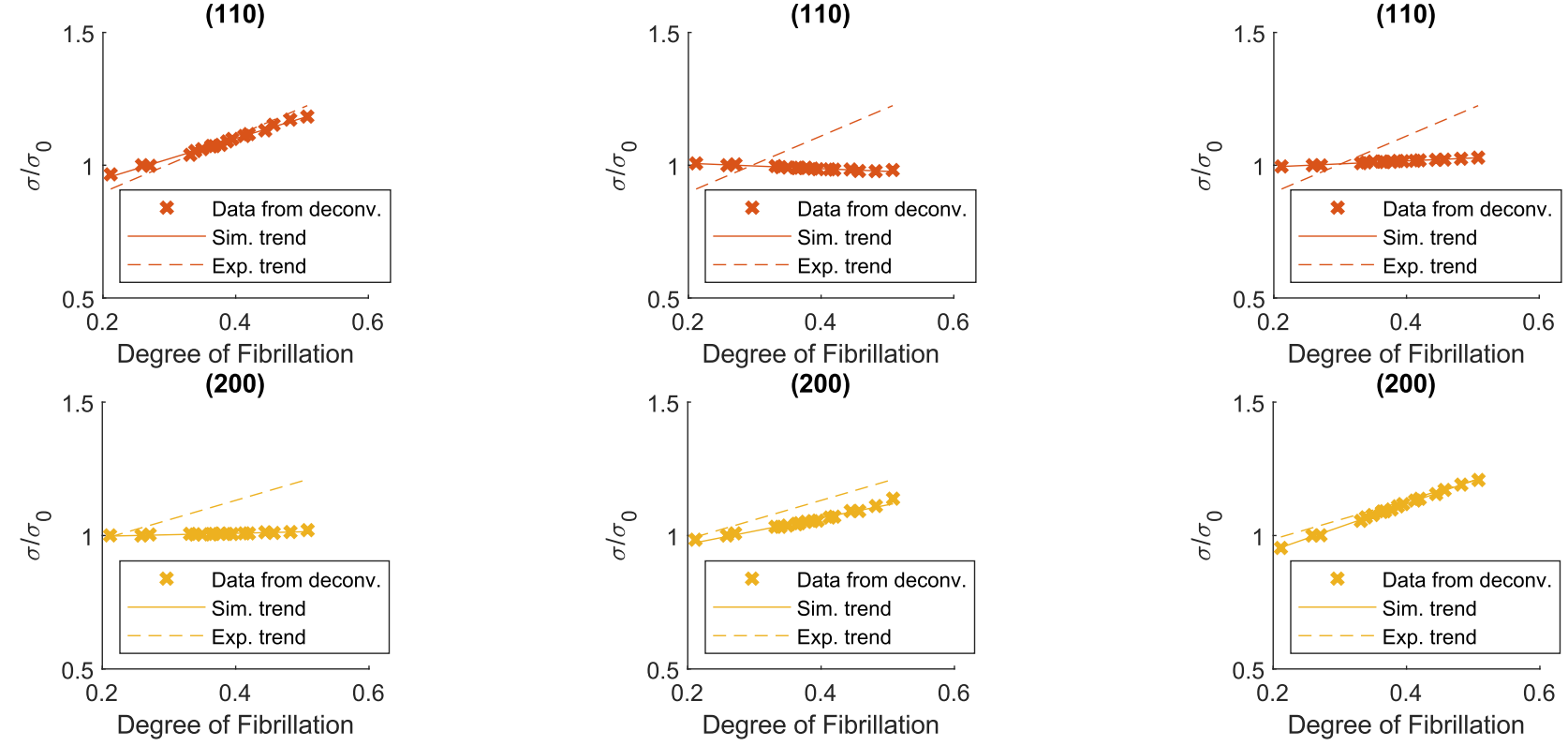


\section{WAXD simulations: Results}

Form factor of a CNF chain with semi-axes

$2 \AA \times 0.75 \AA$ 
Model: H1+

Aggregation direction: (1-10)

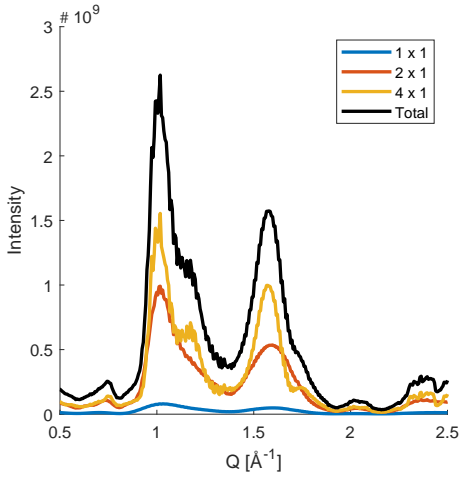

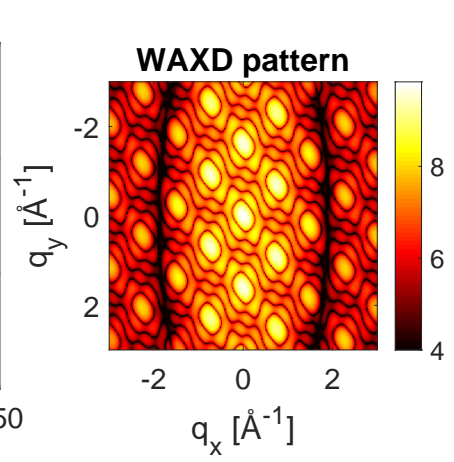

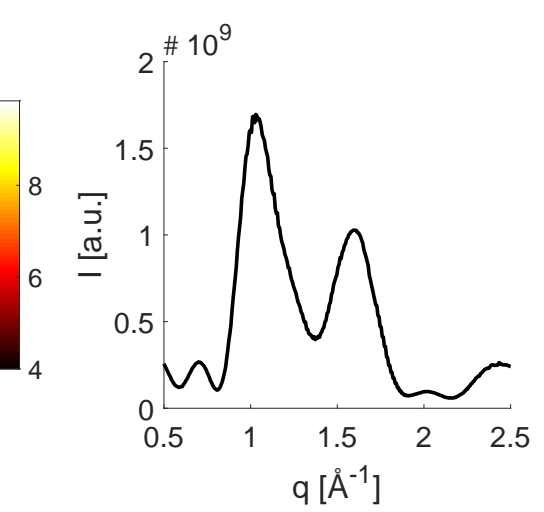

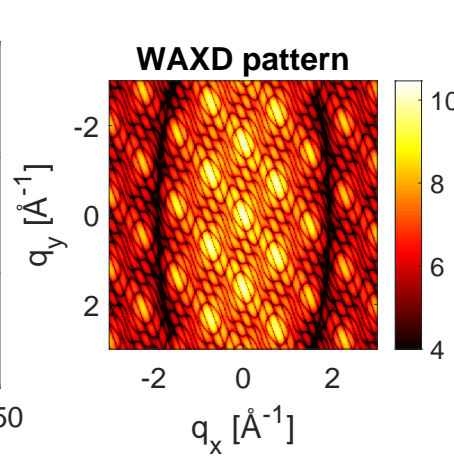

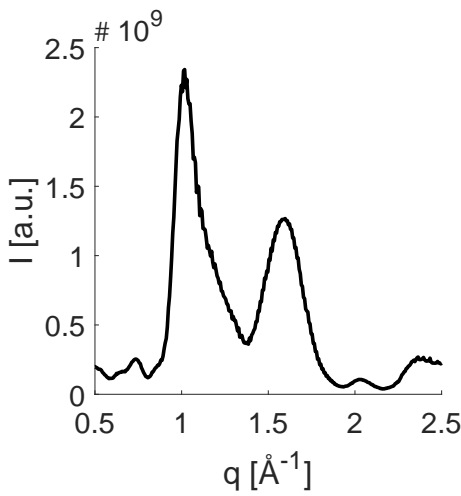

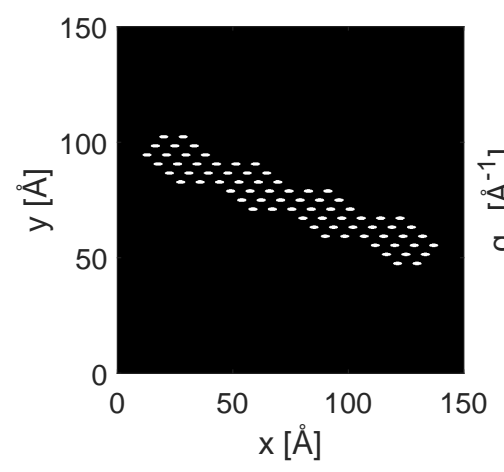
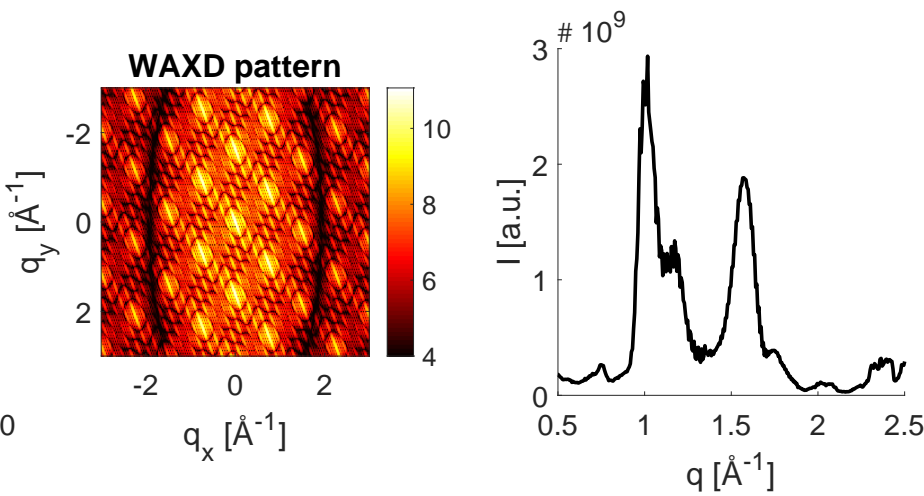

Vol\% 1x1: 4.7

Vol\% $2 \times 1: 42.3$

Vol\% $4 \times 1: 53.0$

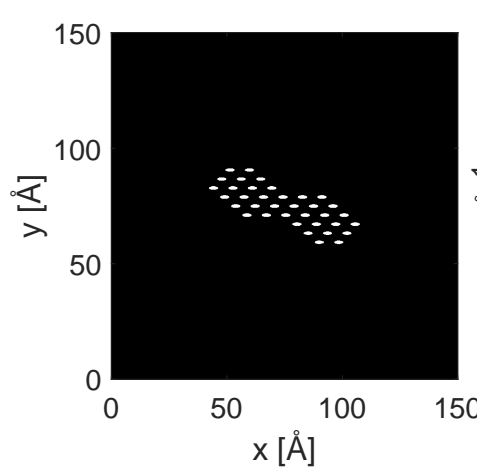


Model: $\mathrm{H} 1+$

Aggregation direction: (110)
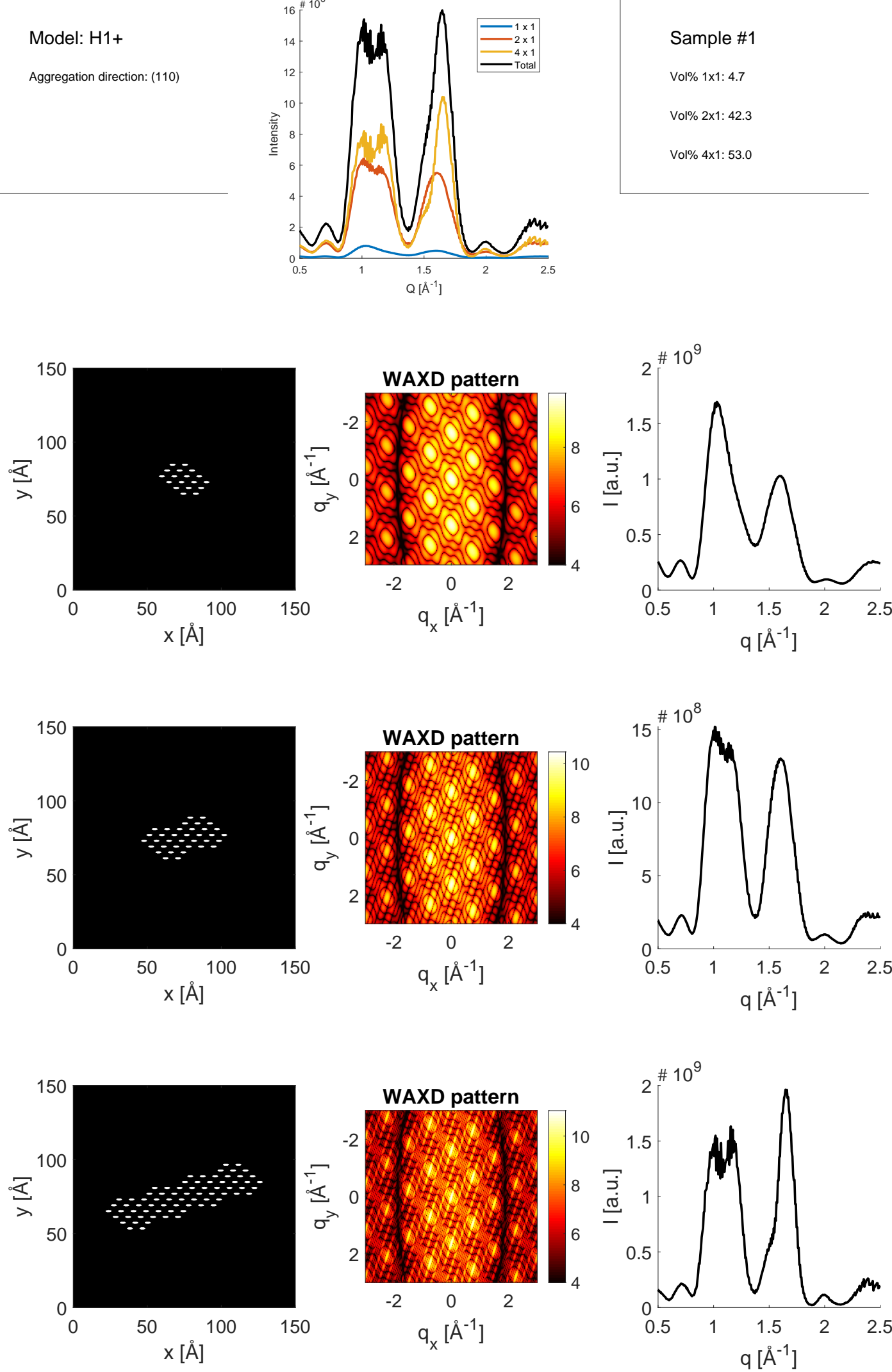

Sample \#1

Vol\% 1x1: 4.7

Vol\% 2x1: 42.3

Vol\% 4x1: 53.0
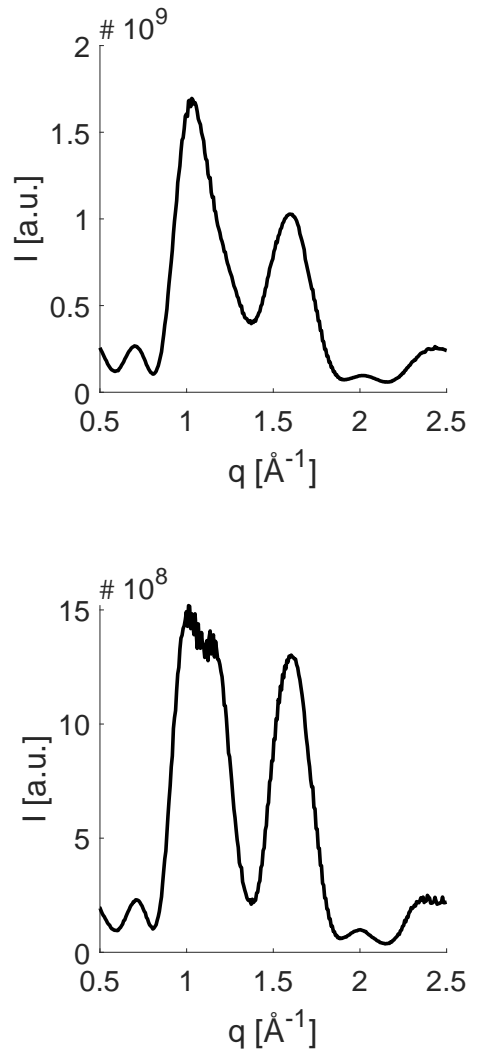

$q\left[A^{-1}\right]$ 
Model: $\mathrm{H1}+$

Aggregation direction: (200)

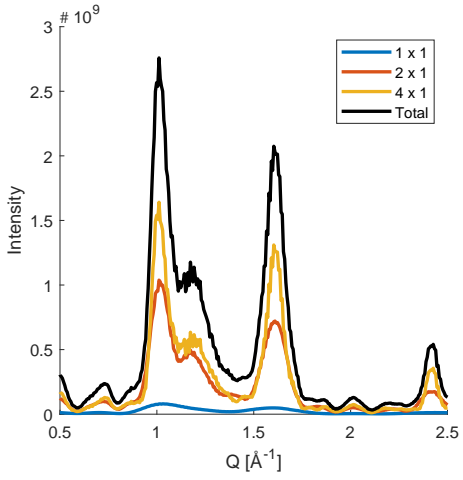

Sample \#1

Vol\% 1x1: 4.7

Vol\% $2 \times 1: 42.3$

Vol\% $4 \times 1: 53.0$
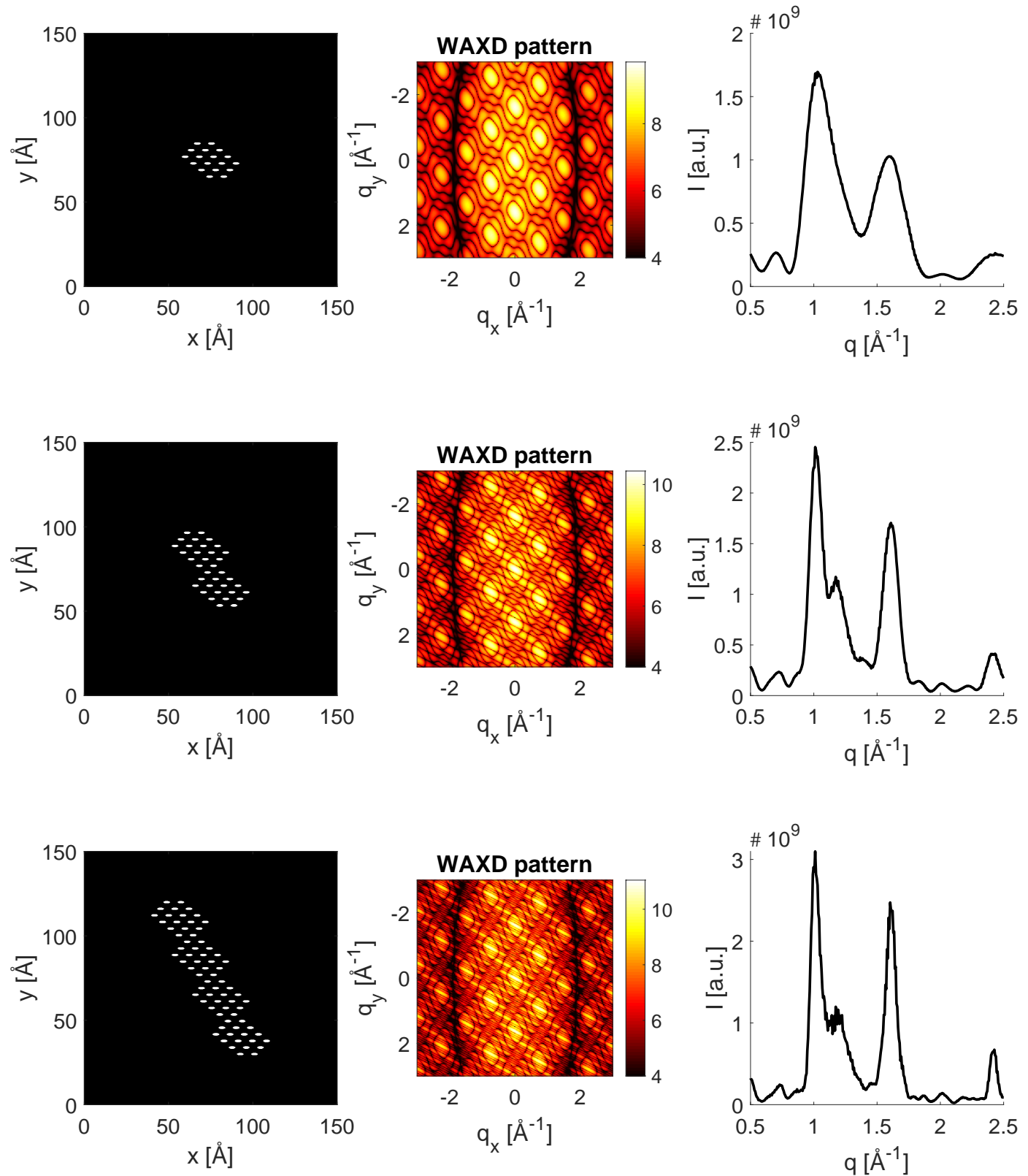
Model: H1-

Aggregation direction: (1-10)

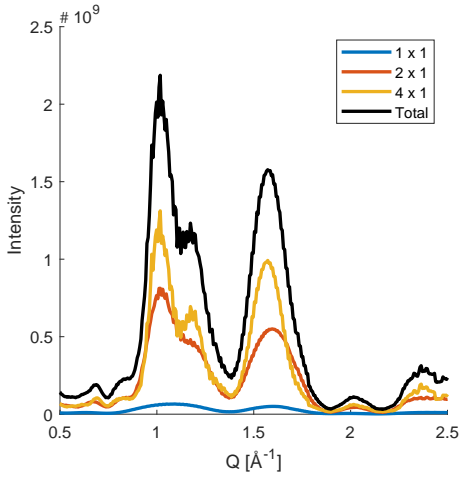

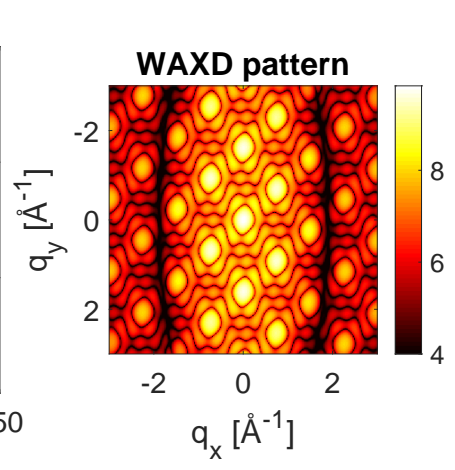

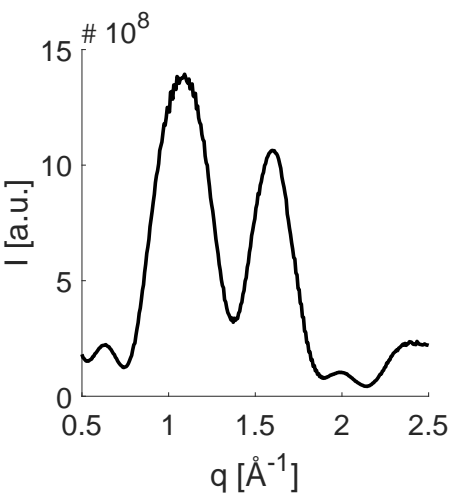

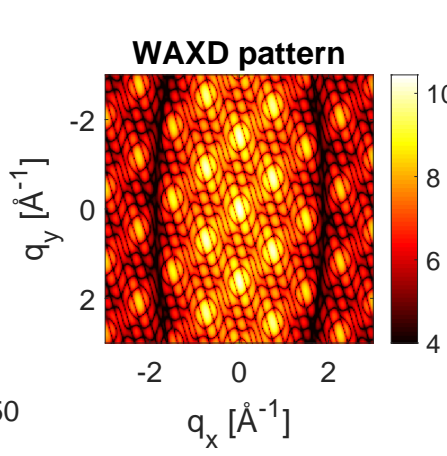

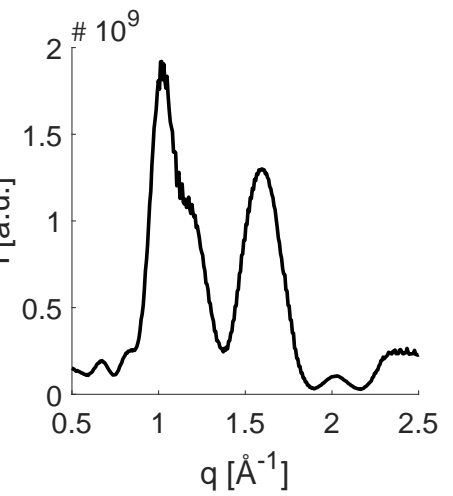

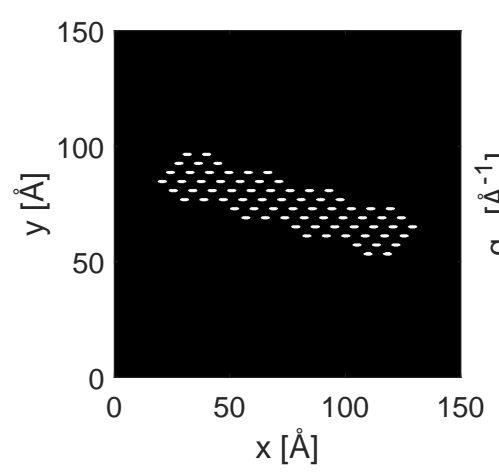
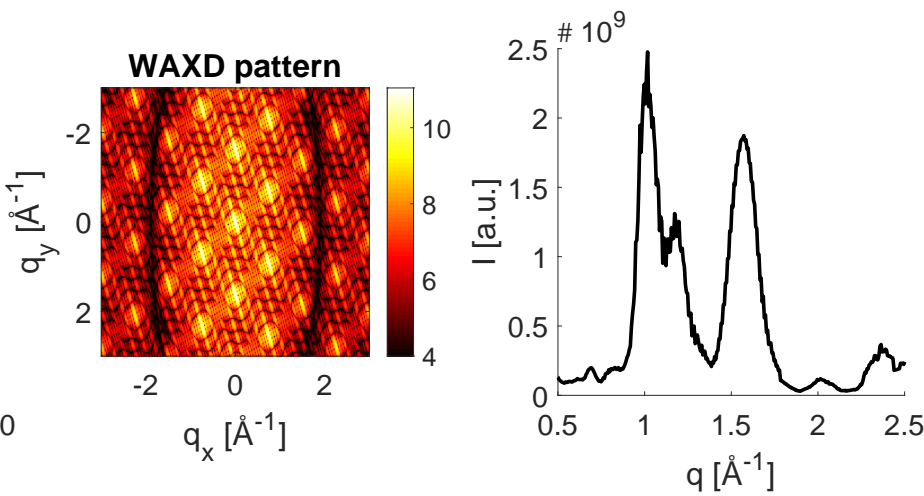
Model: H1-

Aggregation direction: (110)

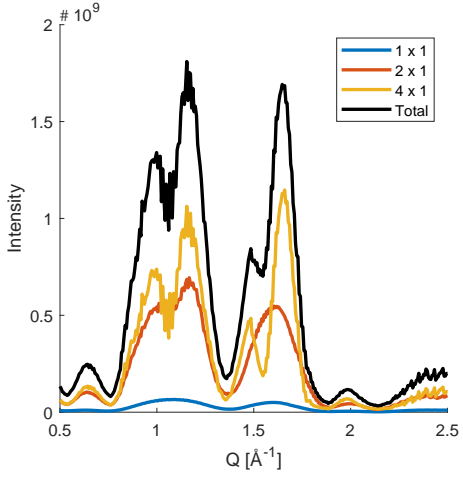

Sample \#1

Vol\% 1x1: 4.7

Vol\% 2x1: 42.3

Vol\% 4x1: 53.0
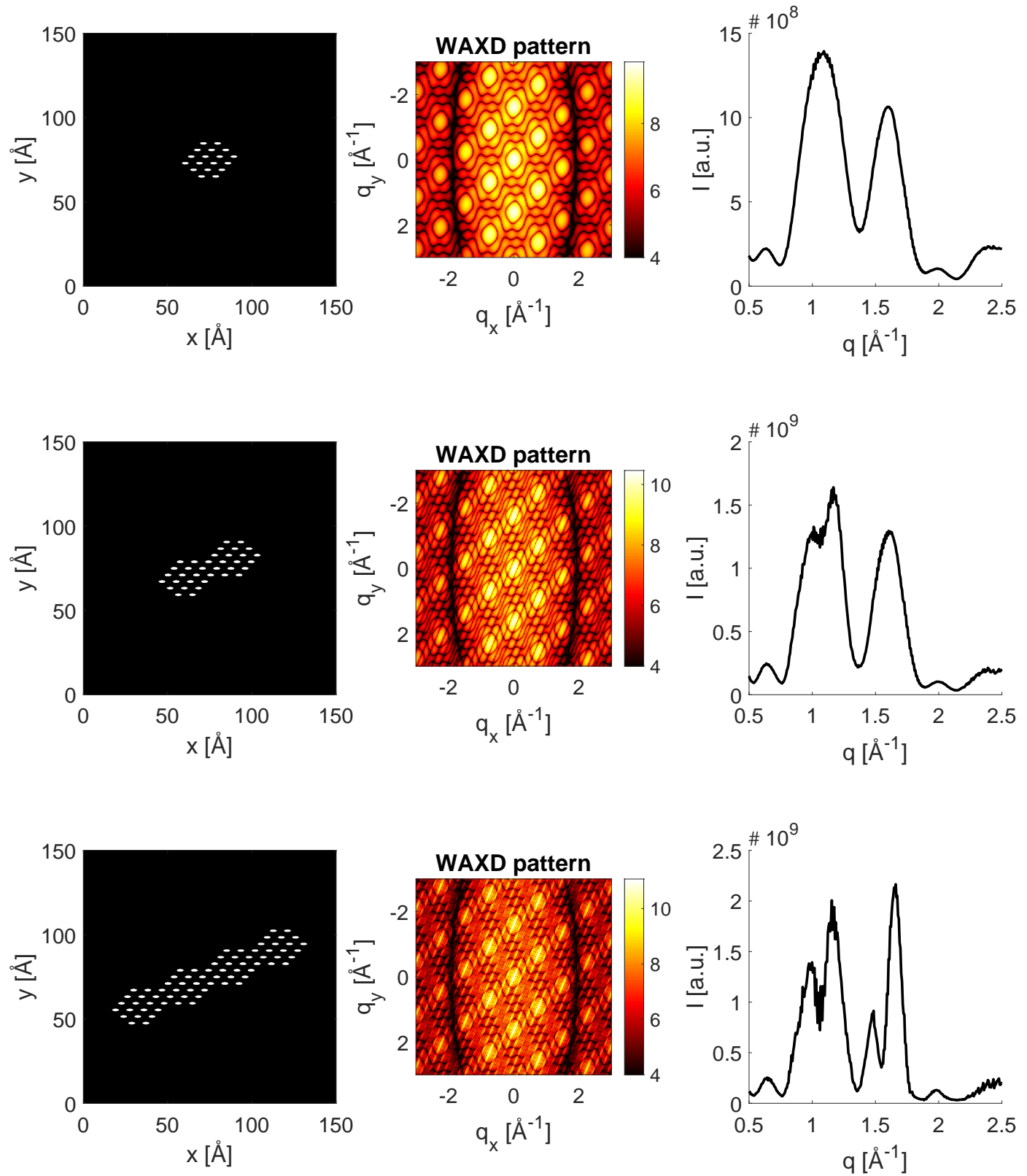
Model: H1-

Aggregation direction: (200)

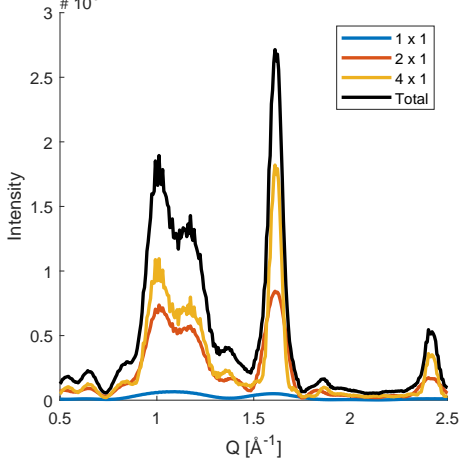

Sample \#1

Vol\% 1x1: 4.7

Vol\% $2 \times 1: 42.3$

Vol\% $4 \times 1: 53.0$
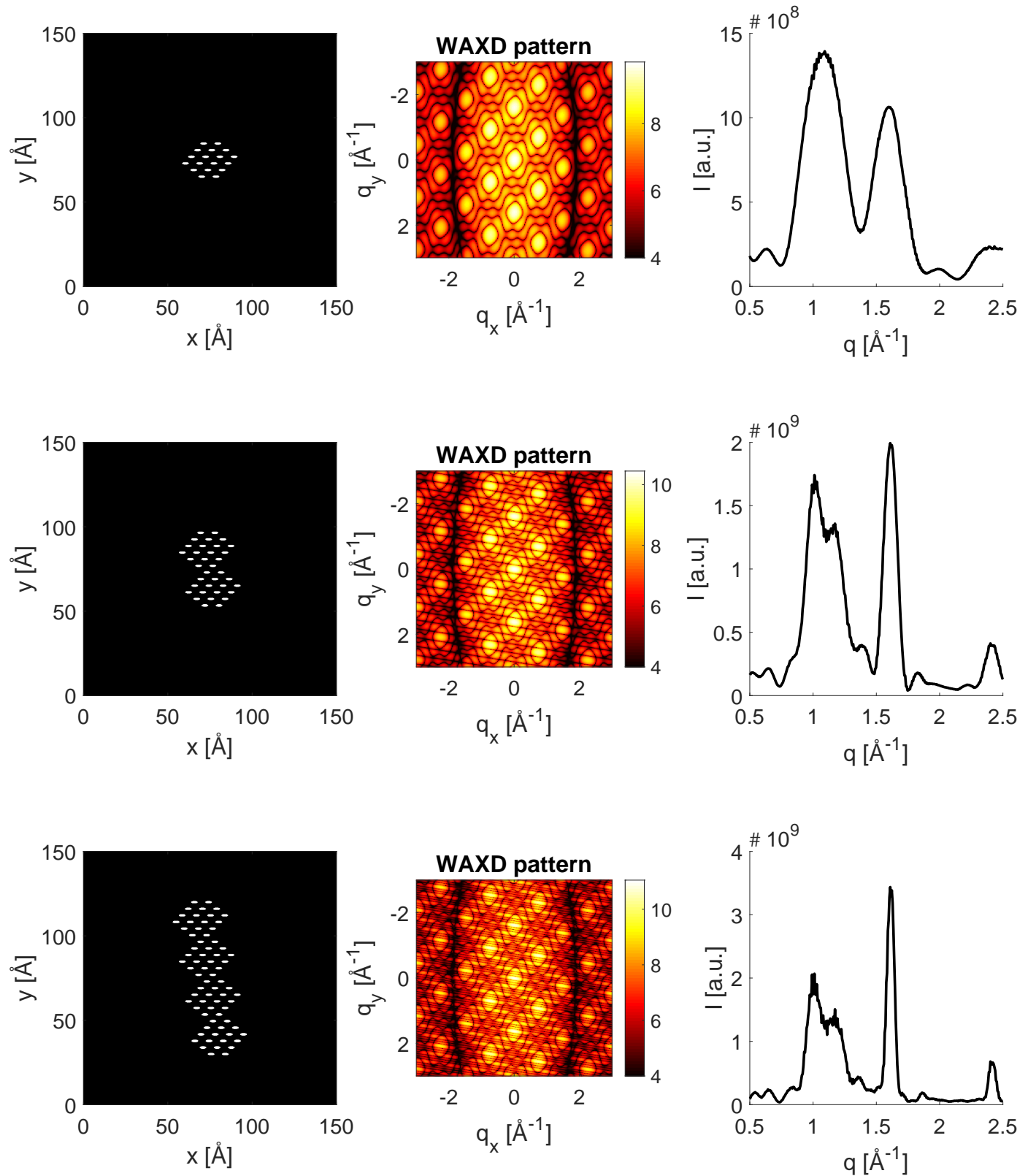
Model: $\mathrm{H} 2+$

Aggregation direction: (1-10)

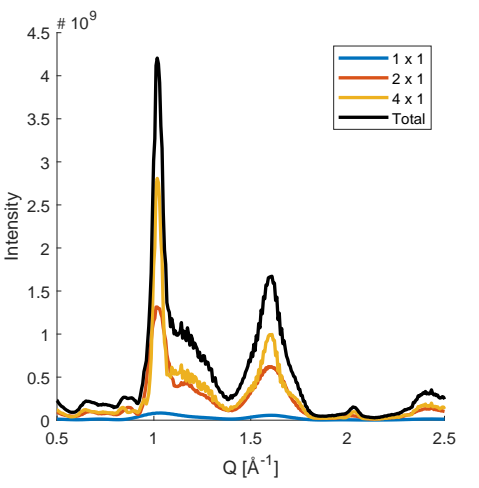

Sample \#1

Vol\% 1x1: 4.7

Vol\% $2 \times 1: 42.3$

Vol\% $4 \times 1: 53.0$
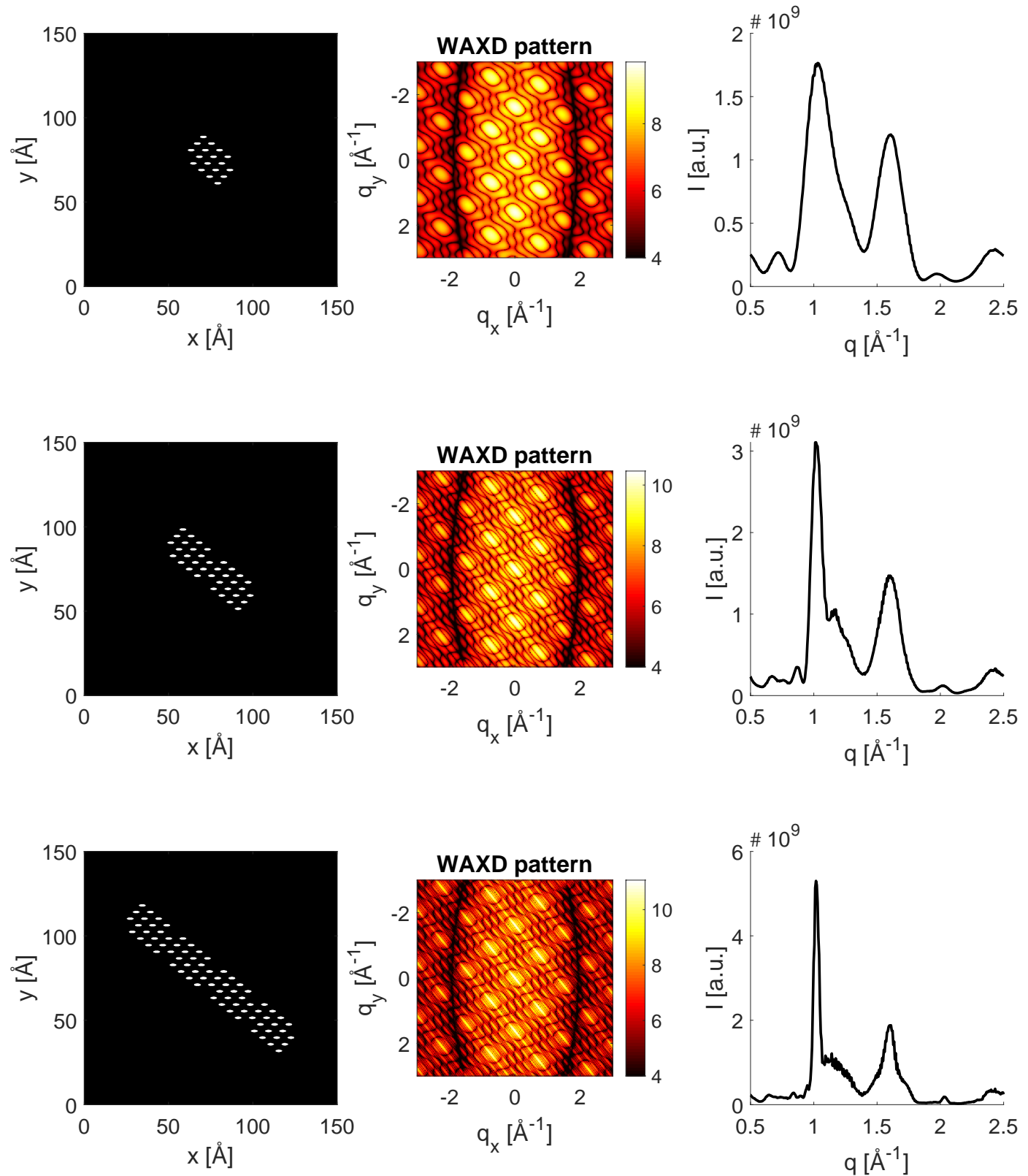
Model: H2+

Aggregation direction: (110)

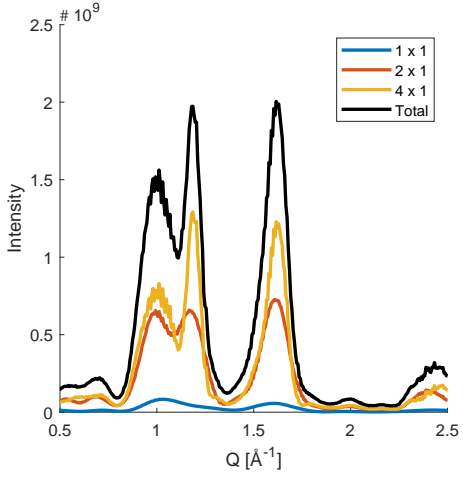

Sample \#1

Vol\% 1x1: 4.7

Vol\% 2x1: 42.3

Vol\% $4 \times 1: 53.0$
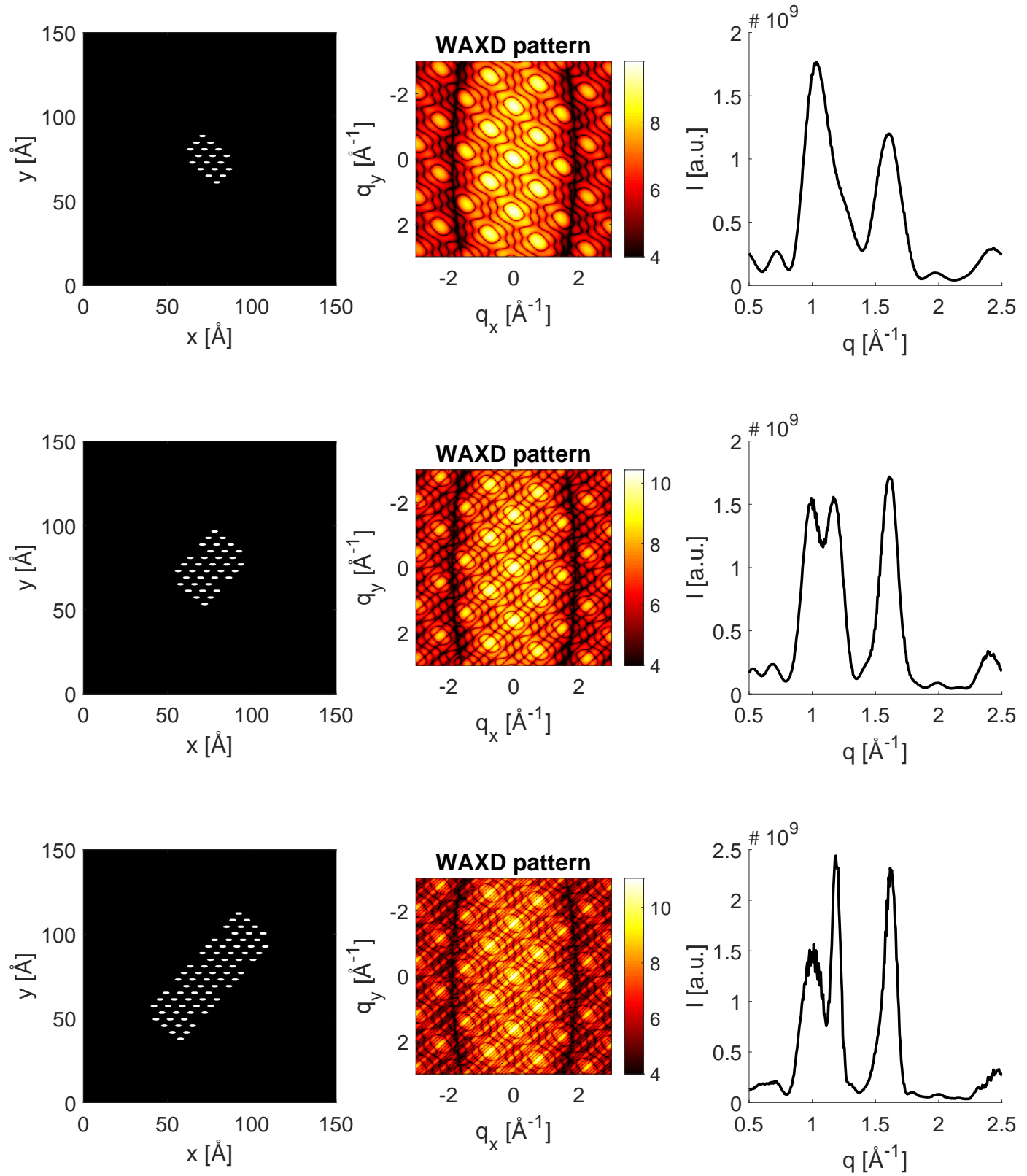
Model: H2-

Aggregation direction: (1-10)

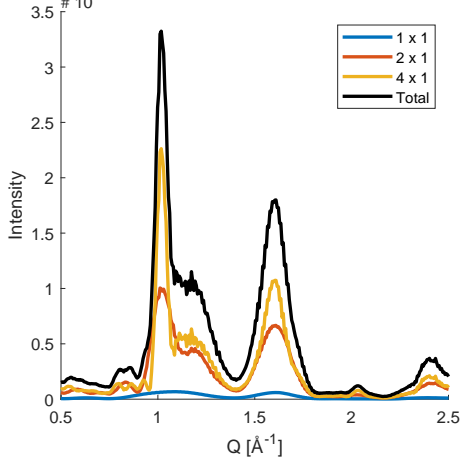

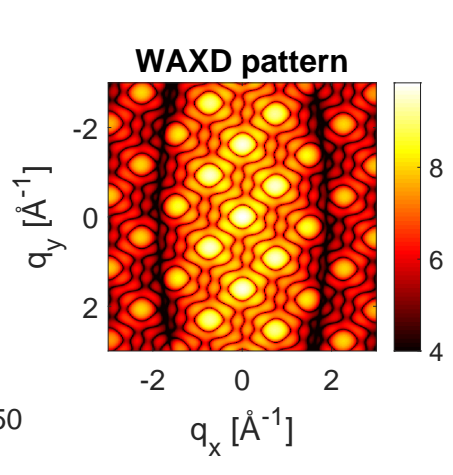
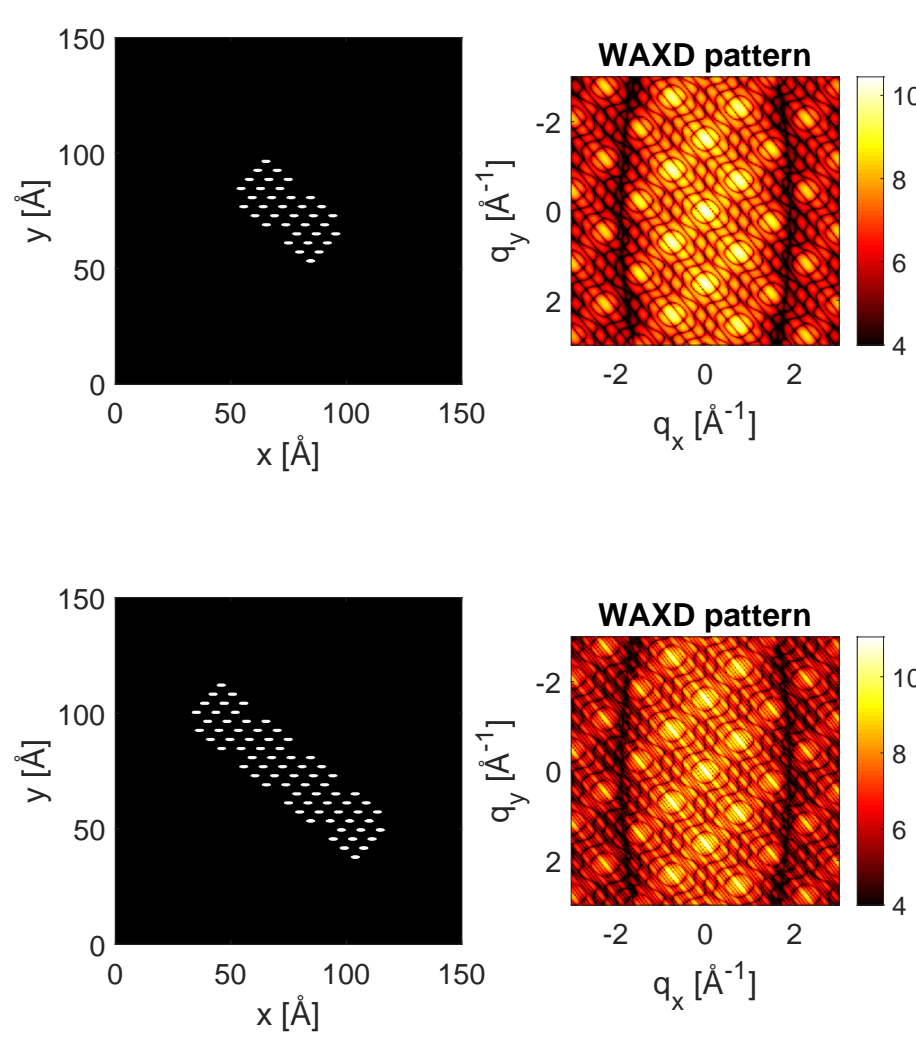

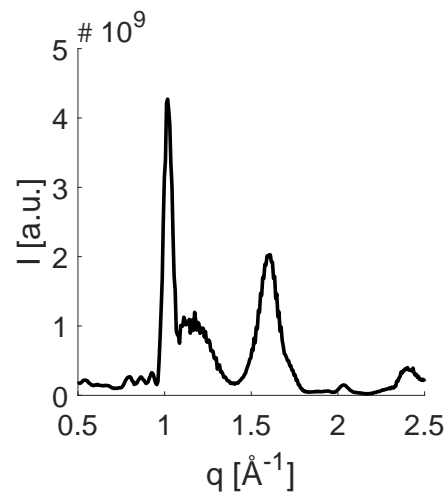

Sample \#1

Vol\% 1x1: 4.7

Vol\% 2x1: 42.3

Vol\% 4x1: 53.0
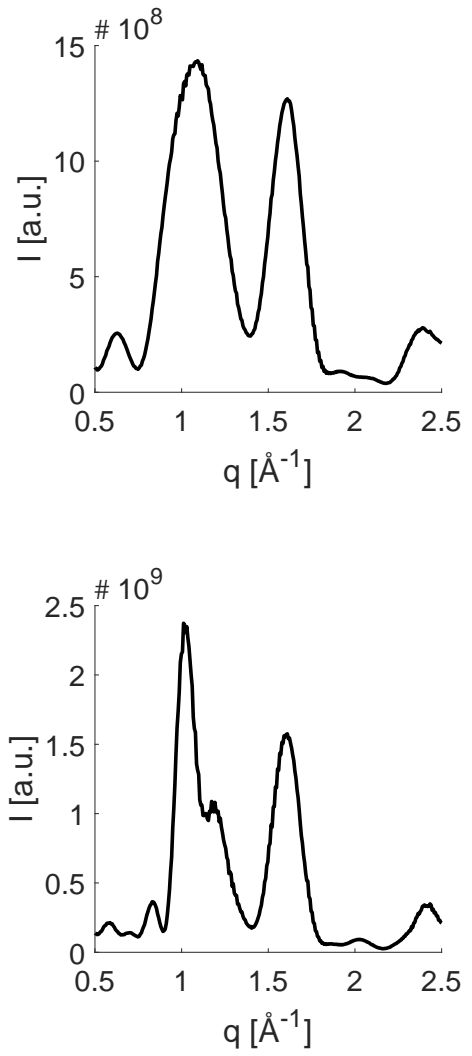

$q\left[A^{-1}\right]$ 
Model: H2-

Aggregation direction: (110)

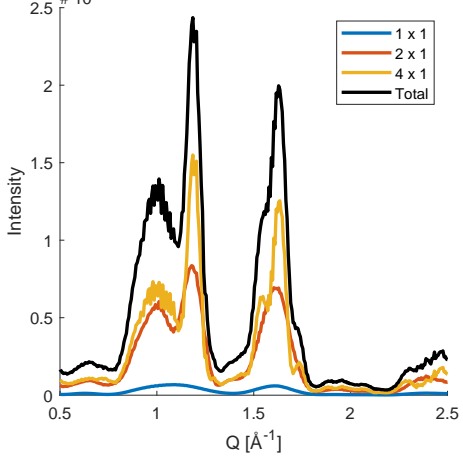

Sample \#1

Vol\% 1x1: 4.7

Vol\% 2x1: 42.3

Vol\% $4 \times 1: 53.0$
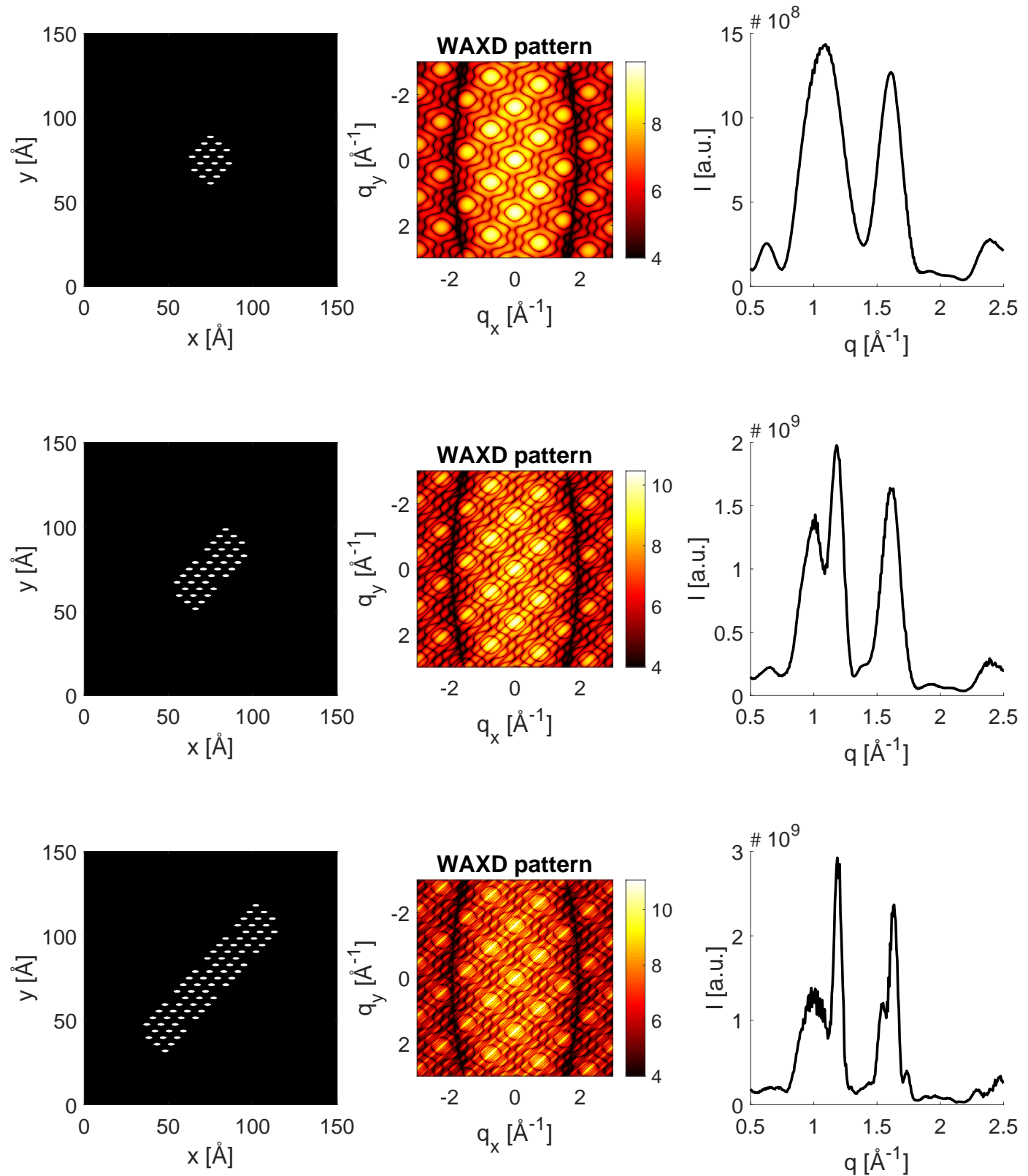
Model: R1+

Aggregation direction: (1-10)

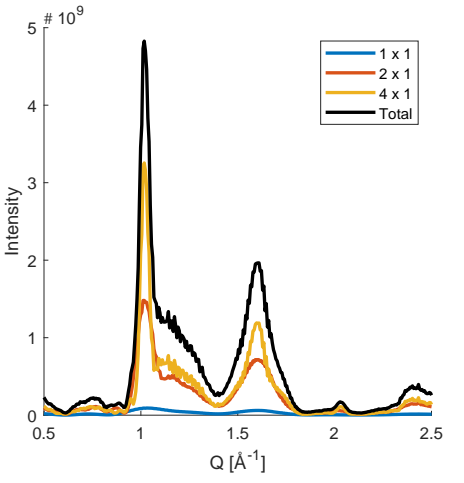

Sample \#1

Vol\% 1x1: 4.7

Vol\% $2 \times 1: 42.3$

Vol\% 4x1: 53.0
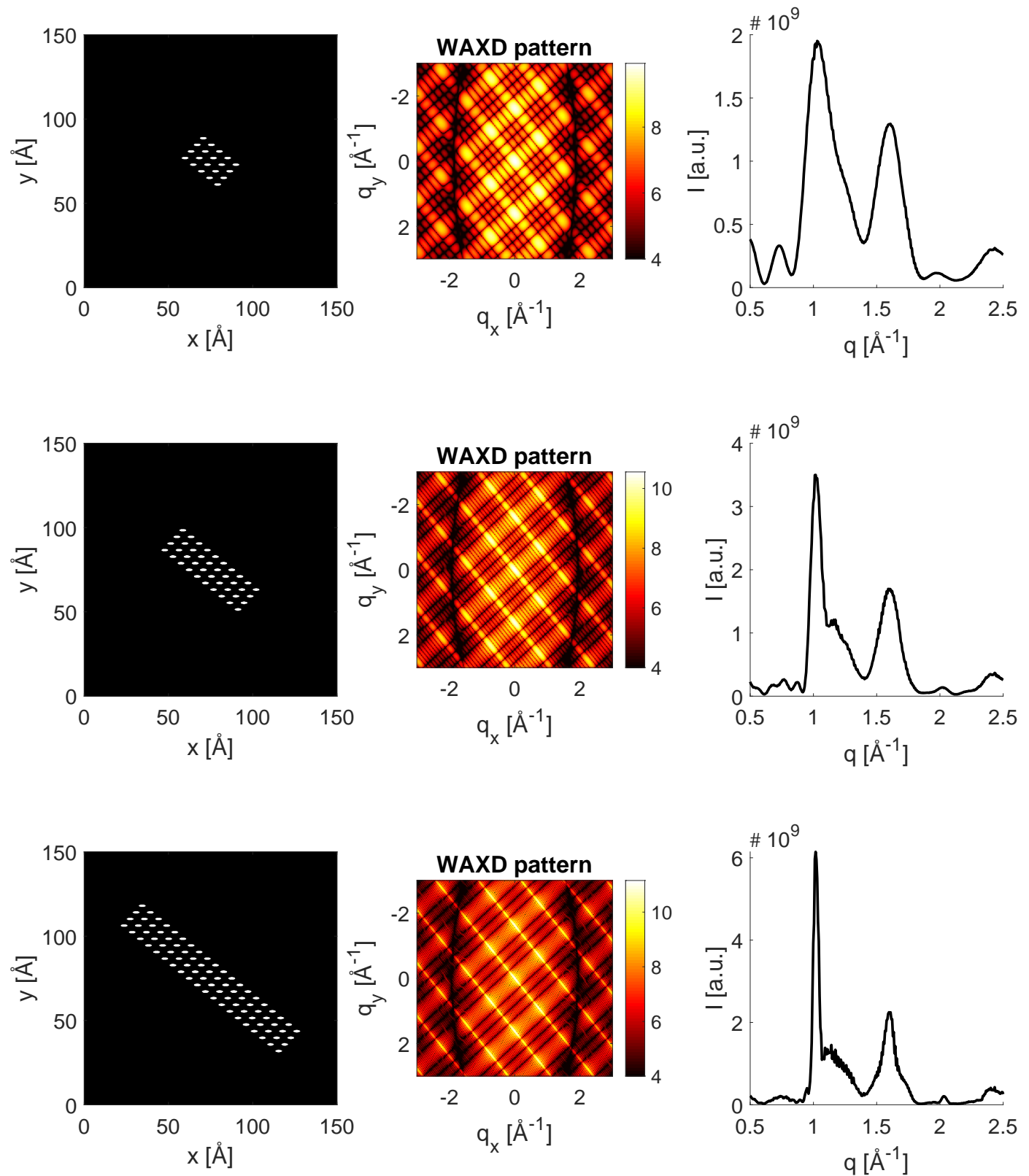
Model: R1+

Aggregation direction: (110)

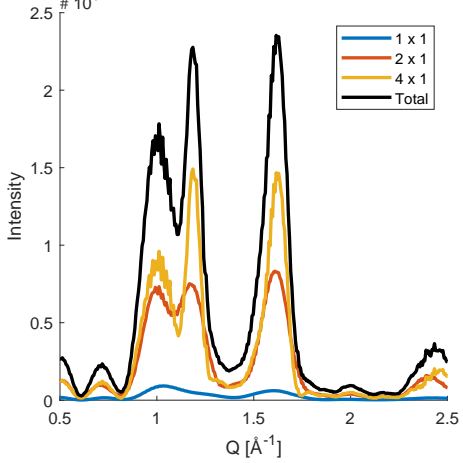

Sample \#1

Vol\% 1x1: 4.7

Vol\% 2x1: 42.3

Vol\% 4x1: 53.0
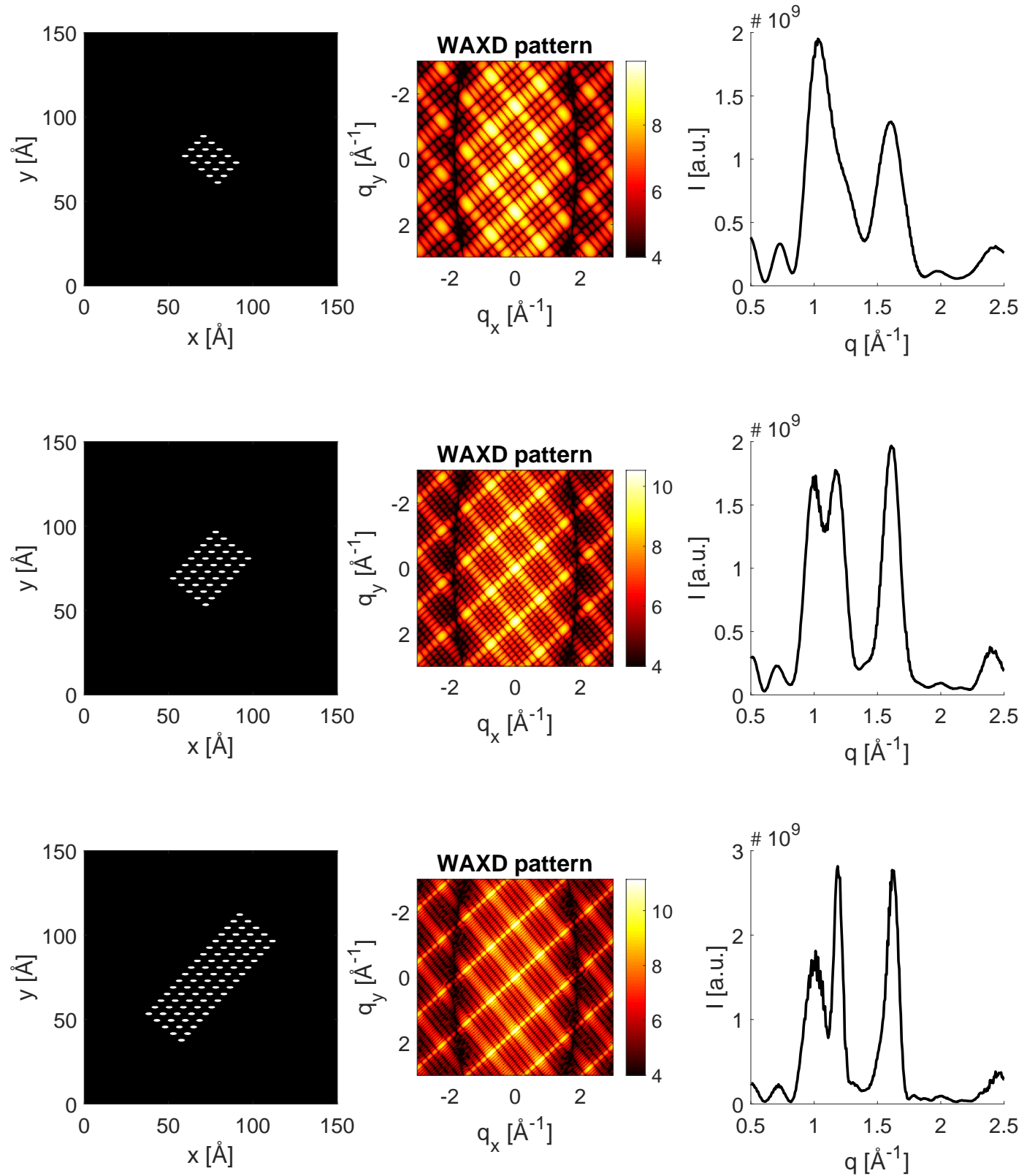
Model: R1-

Aggregation direction: (1-10)

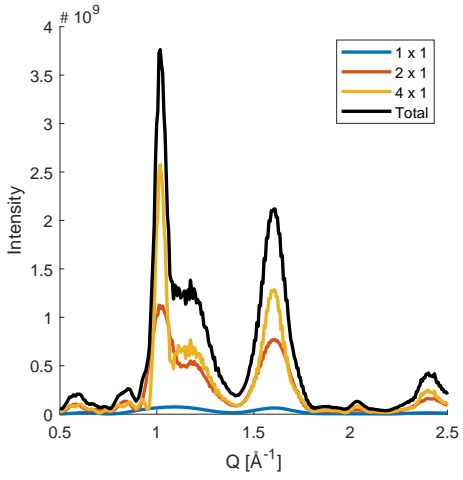

Sample \#1

Vol\% 1x1: 4.7

Vol\% $2 \times 1: 42.3$

Vol\% 4x1: 53.0
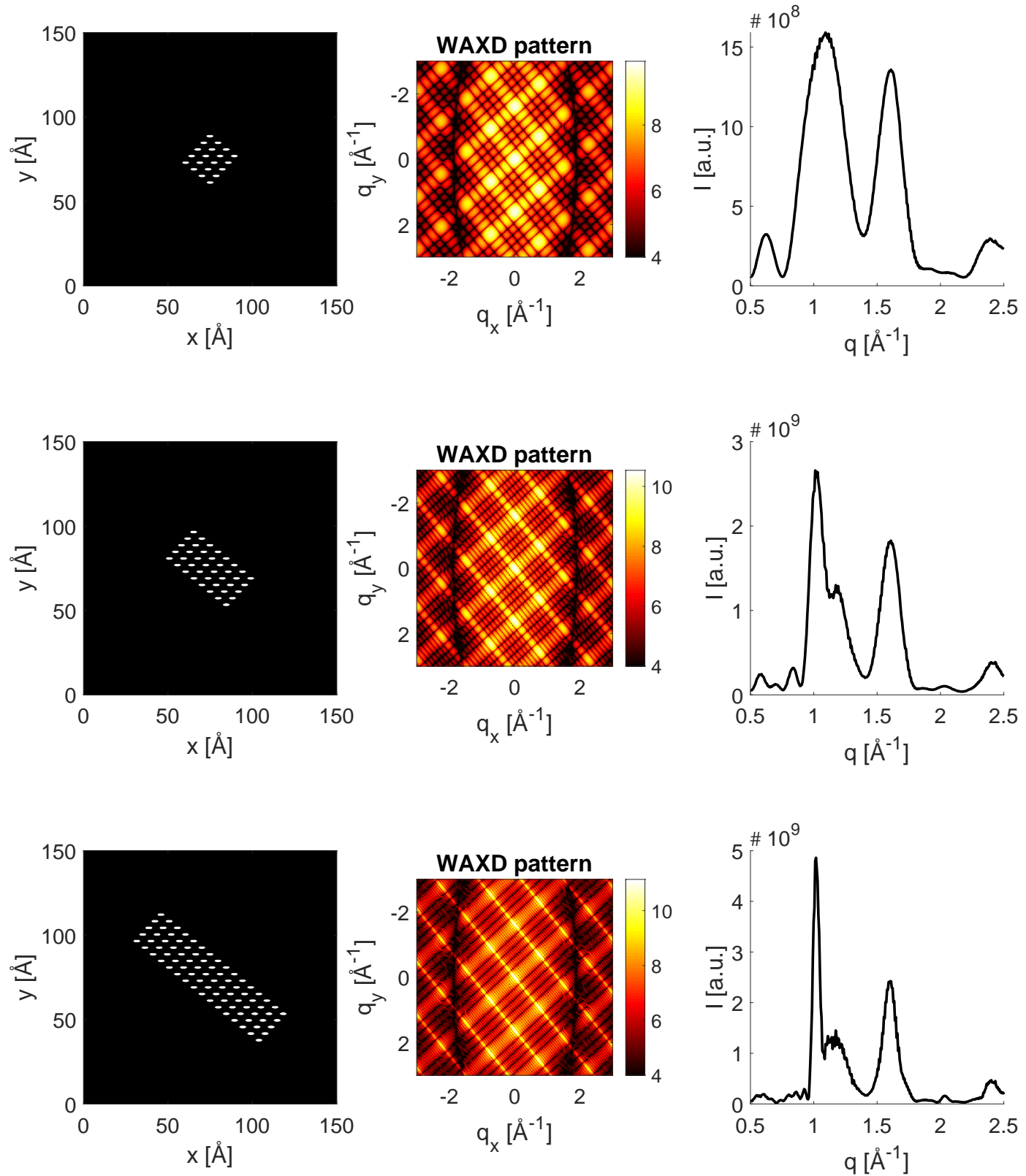
Model: R1-

Aggregation direction: (110)

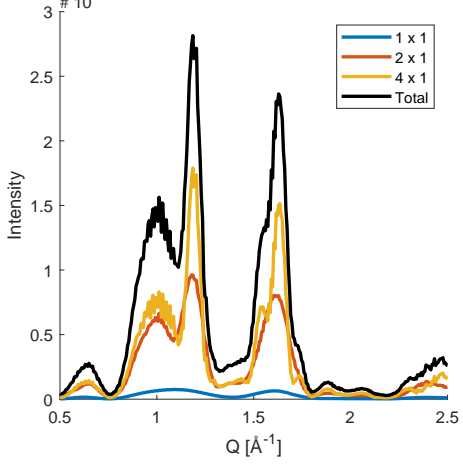

Sample \#1

Vol\% 1x1: 4.7

Vol\% $2 \times 1: 42.3$

Vol\% $4 \times 1: 53.0$
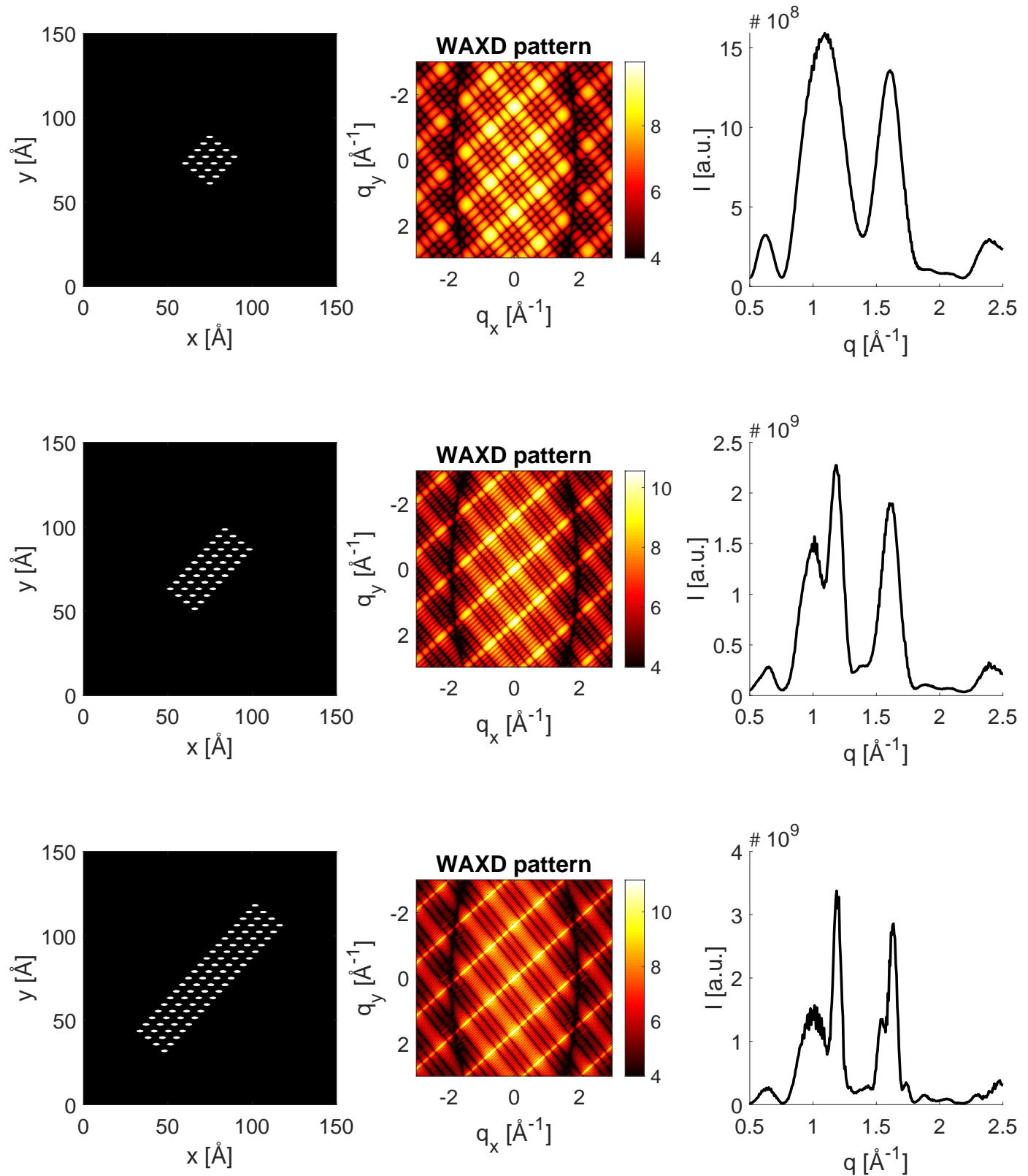
Model: R2+

Aggregation direction: (1-10)

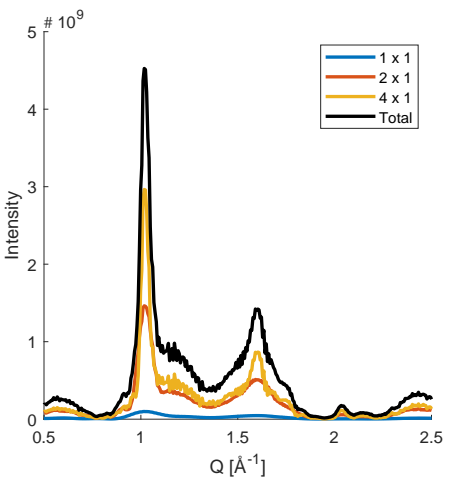

Sample \#1

Vol\% 1x1: 4.7

Vol\% $2 \times 1: 42.3$

Vol\% $4 \times 1: 53.0$
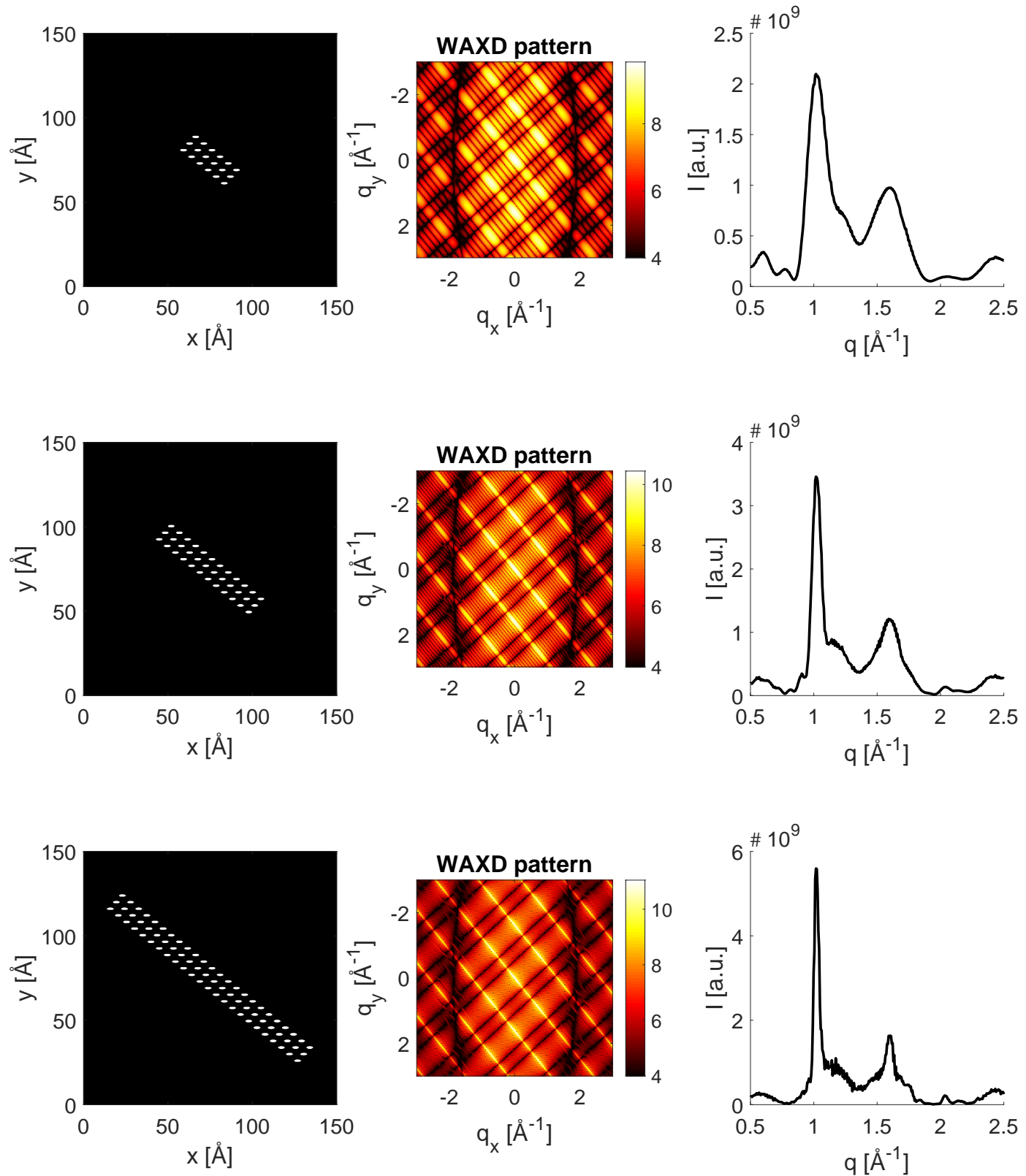
Model: R2+

Aggregation direction: (110)

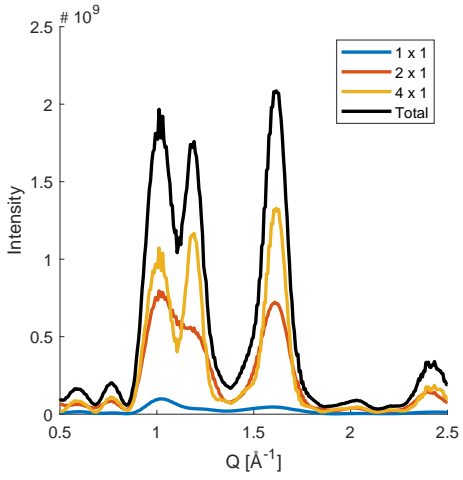

Sample \#1

Vol\% 1x1: 4.7

Vol\% 2x1: 42.3

Vol\% 4x1: 53.0
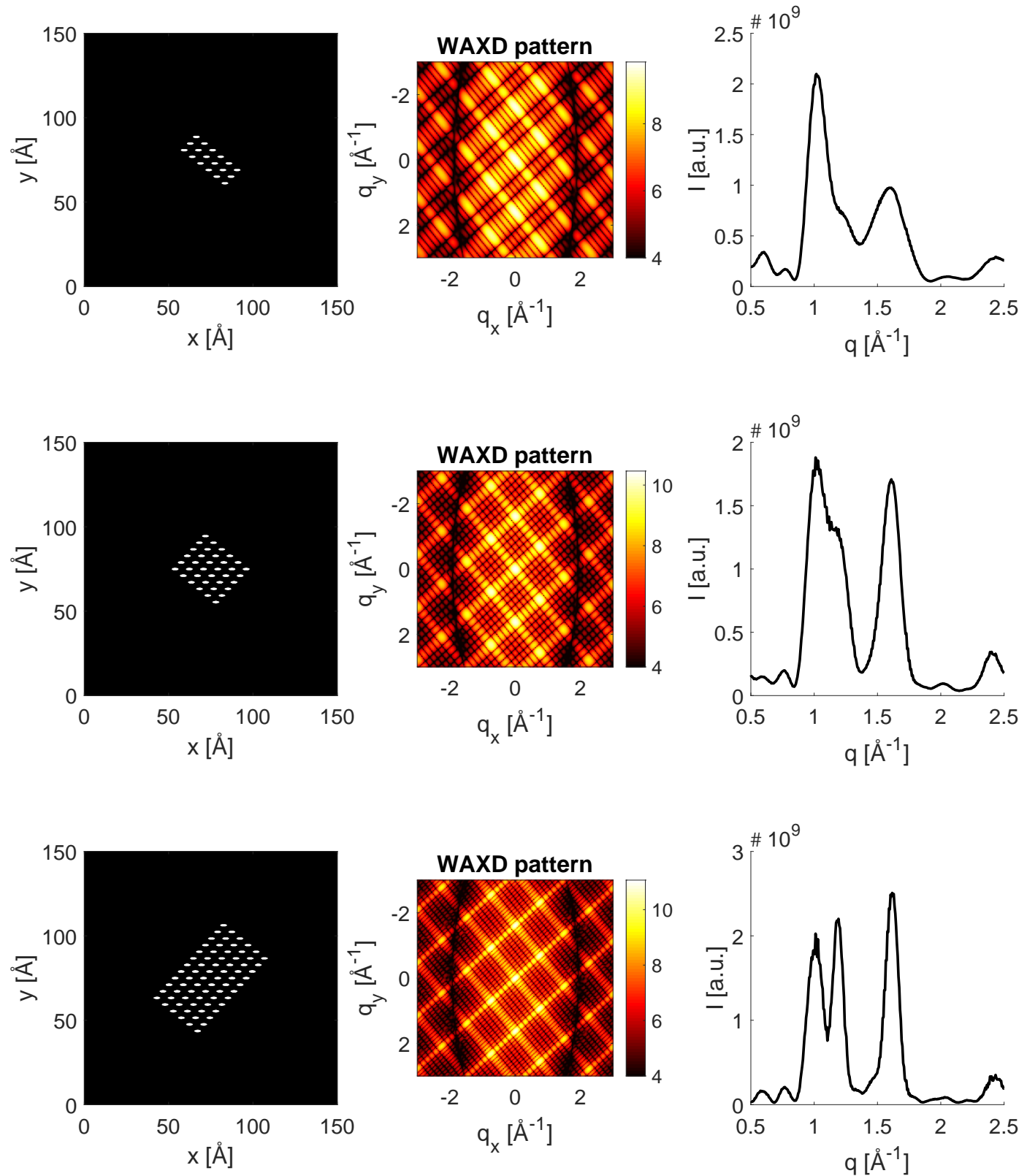
Model: R2-

Aggregation direction: (1-10)

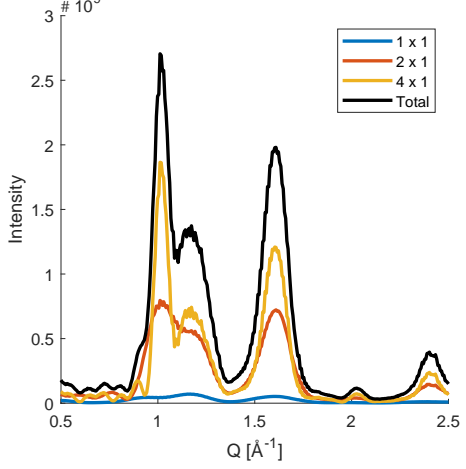

Sample \#1

Vol\% 1x1: 4.7

Vol\% 2x1: 42.3

Vol\% $4 \times 1: 53.0$
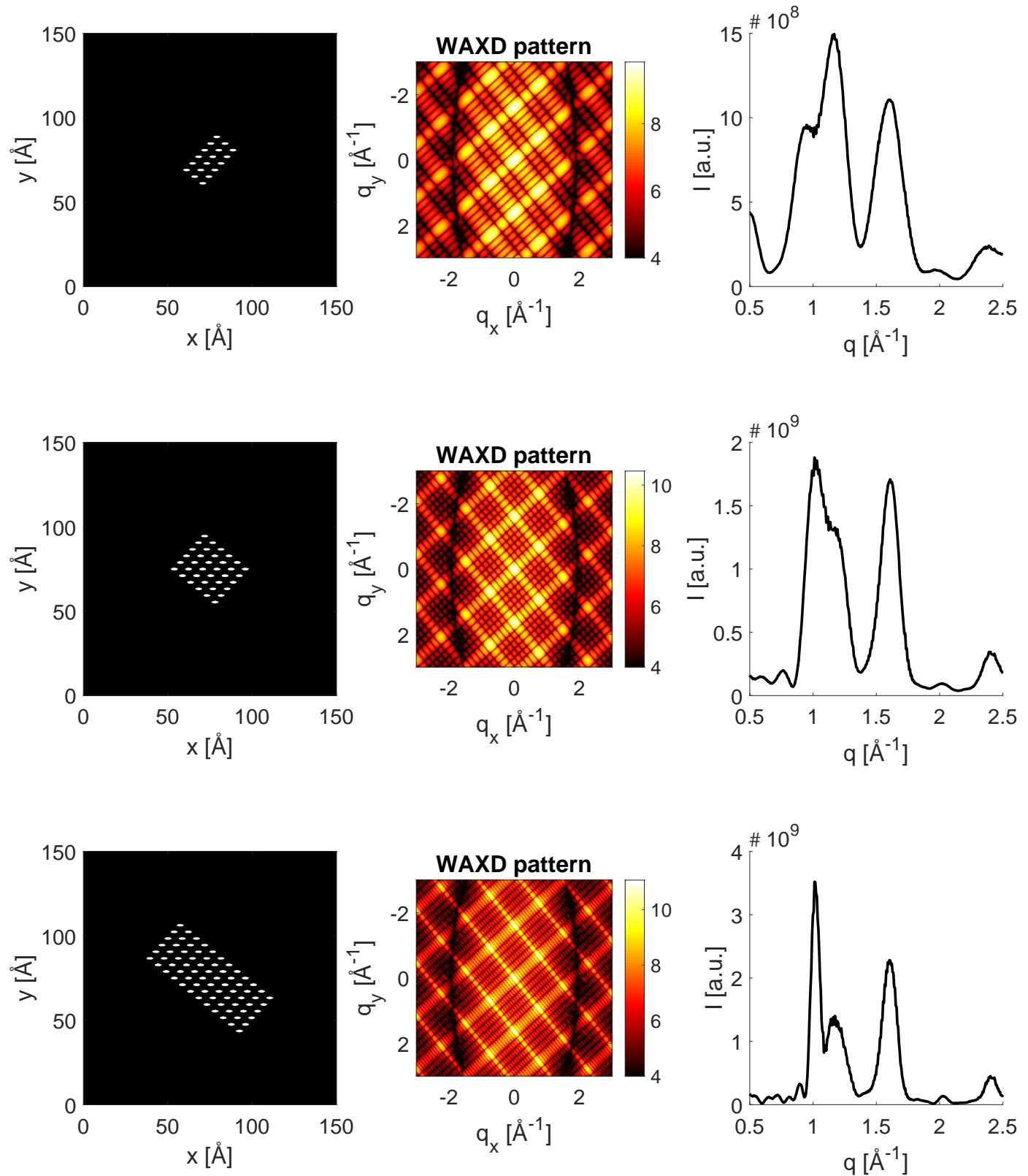
Model: R2-

Aggregation direction: (110)

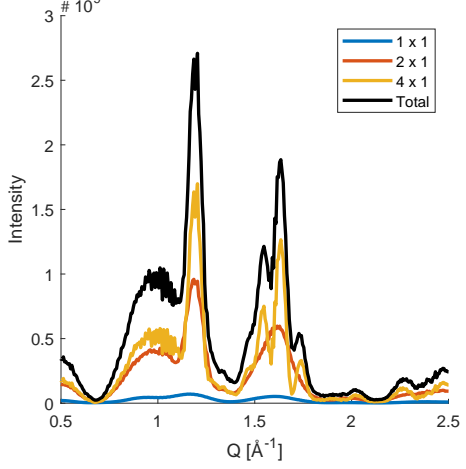

Sample \#1

Vol\% 1x1: 4.7

Vol\% 2x1: 42.3

Vol\% $4 \times 1: 53.0$
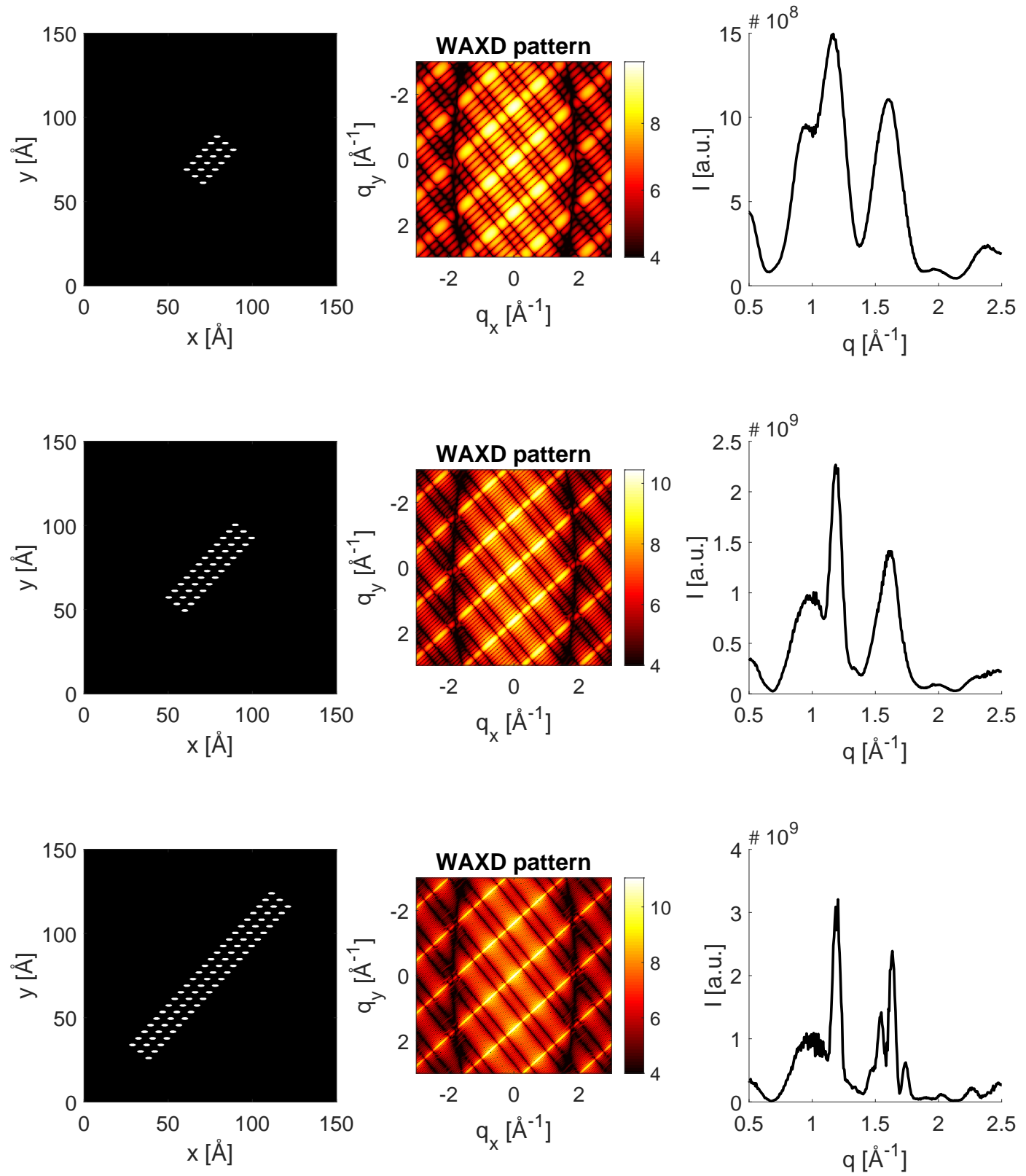
Model: Rh1+

Aggregation direction: (110)
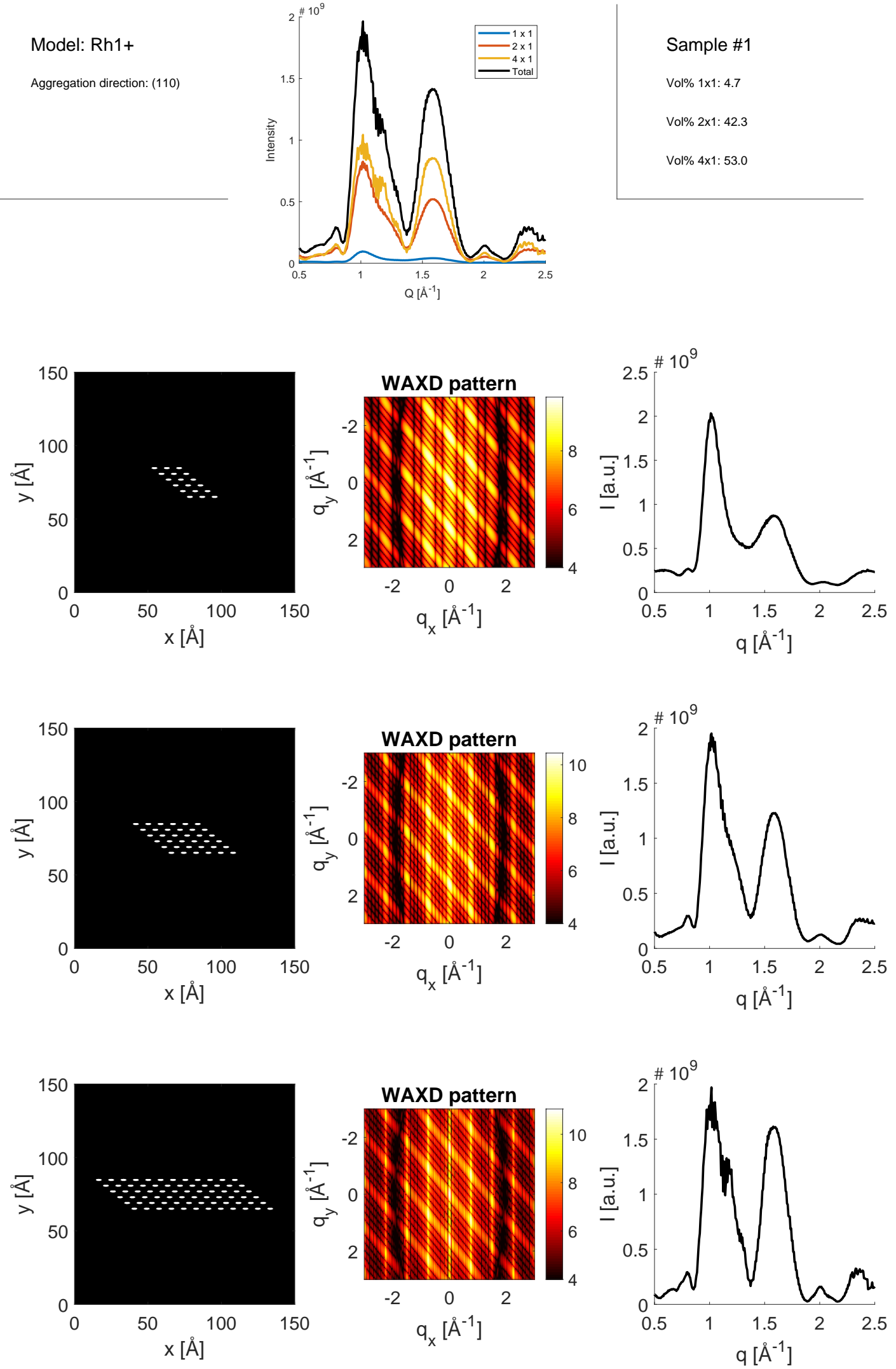

Sample \#1

Vol\% 1 1 1: 4.7

Vol\% 2x1: 42.3

Vol\% 4x1: 53.0

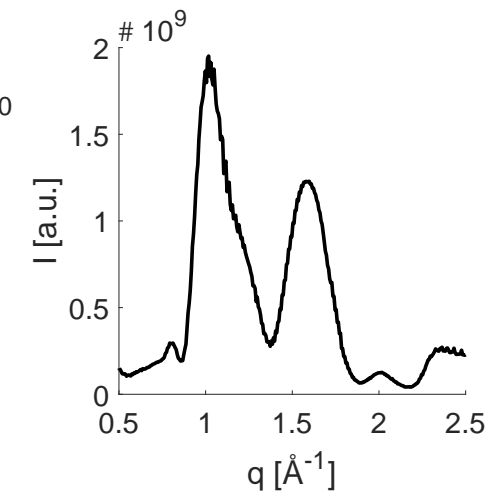


Model: Rh1+

Aggregation direction: (200)

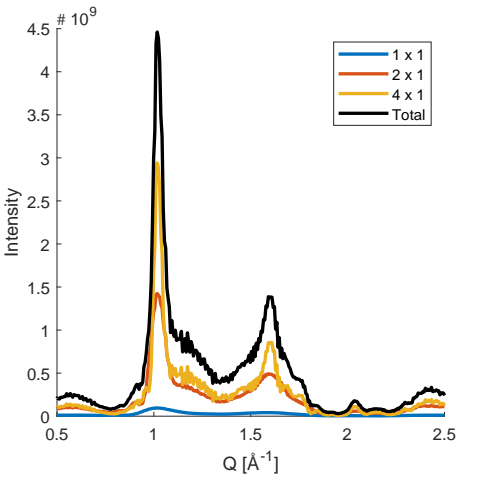

Sample \#1

Vol\% 1x1: 4.7

Vol\% $2 \times 1: 42.3$

Vol\% 4x1: 53.0
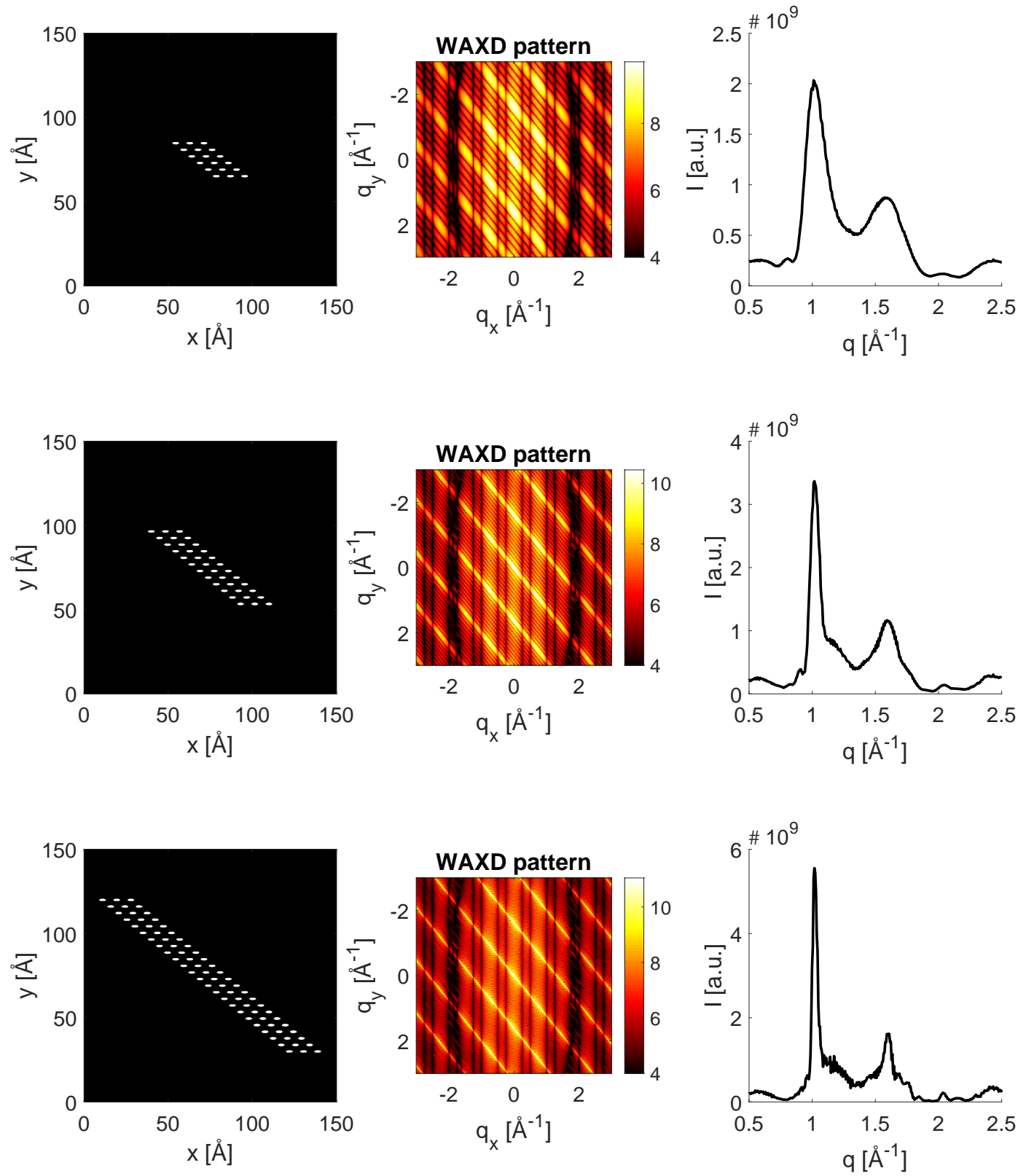
Model: Rh1-

Aggregation direction: (1-10)

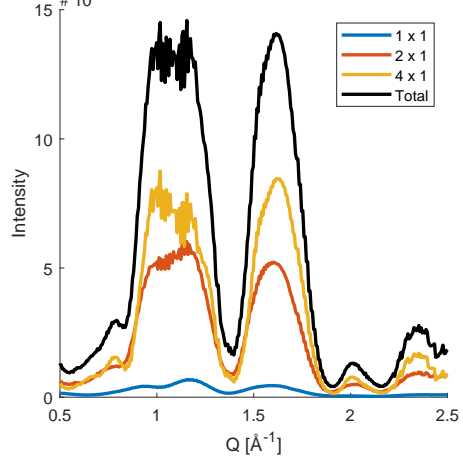

Sample \#1

Vol\% 1x1: 4.7

Vol\% 2x1: 42.3

Vol\% 4x1: 53.0
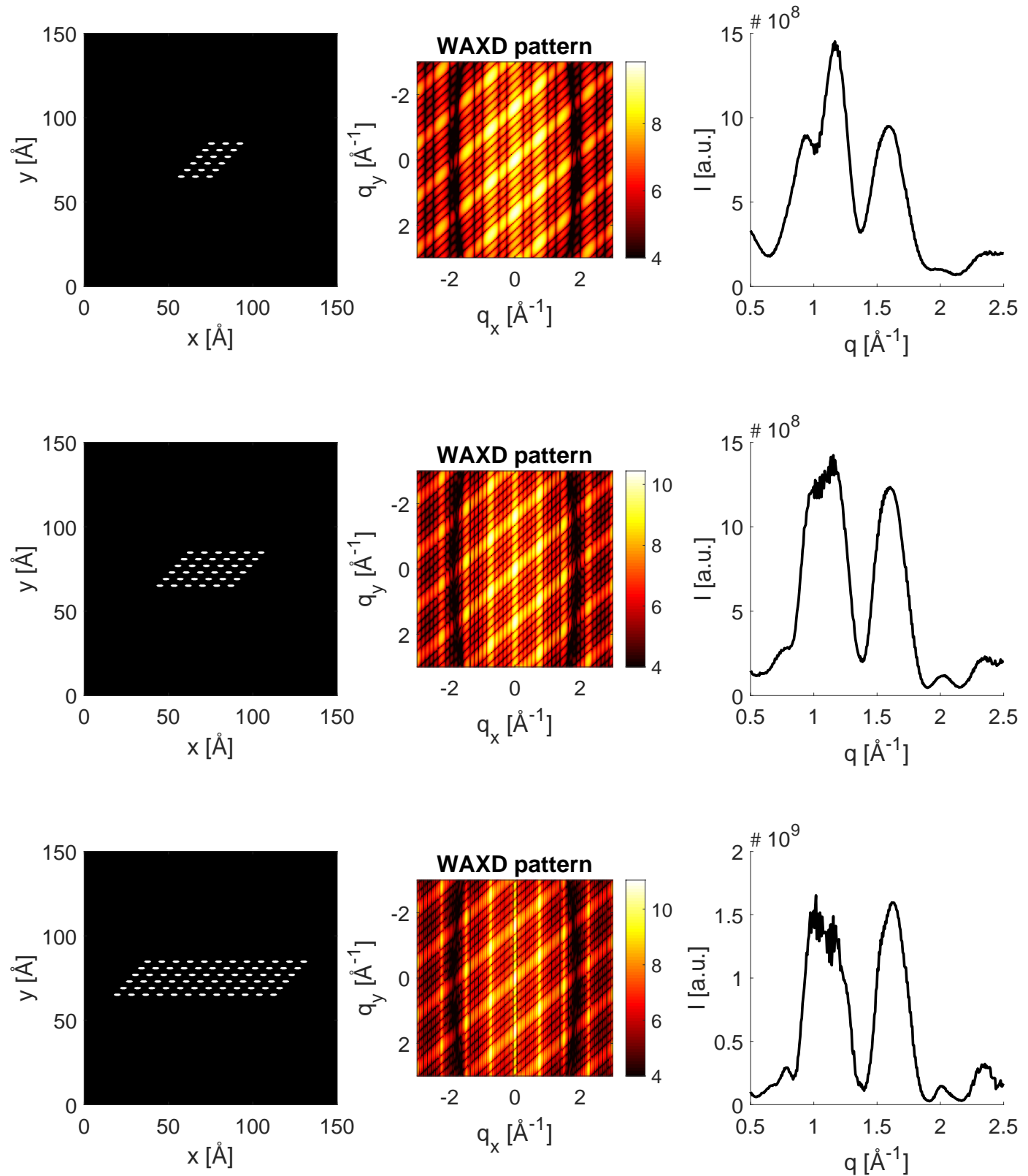
Model: Rh1-

Aggregation direction: (200)

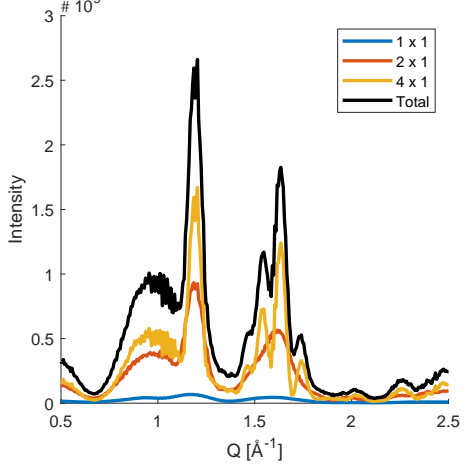

Sample \#1

Vol\% 1x1: 4.7

Vol\% $2 \times 1: 42.3$

Vol\% $4 \times 1: 53.0$
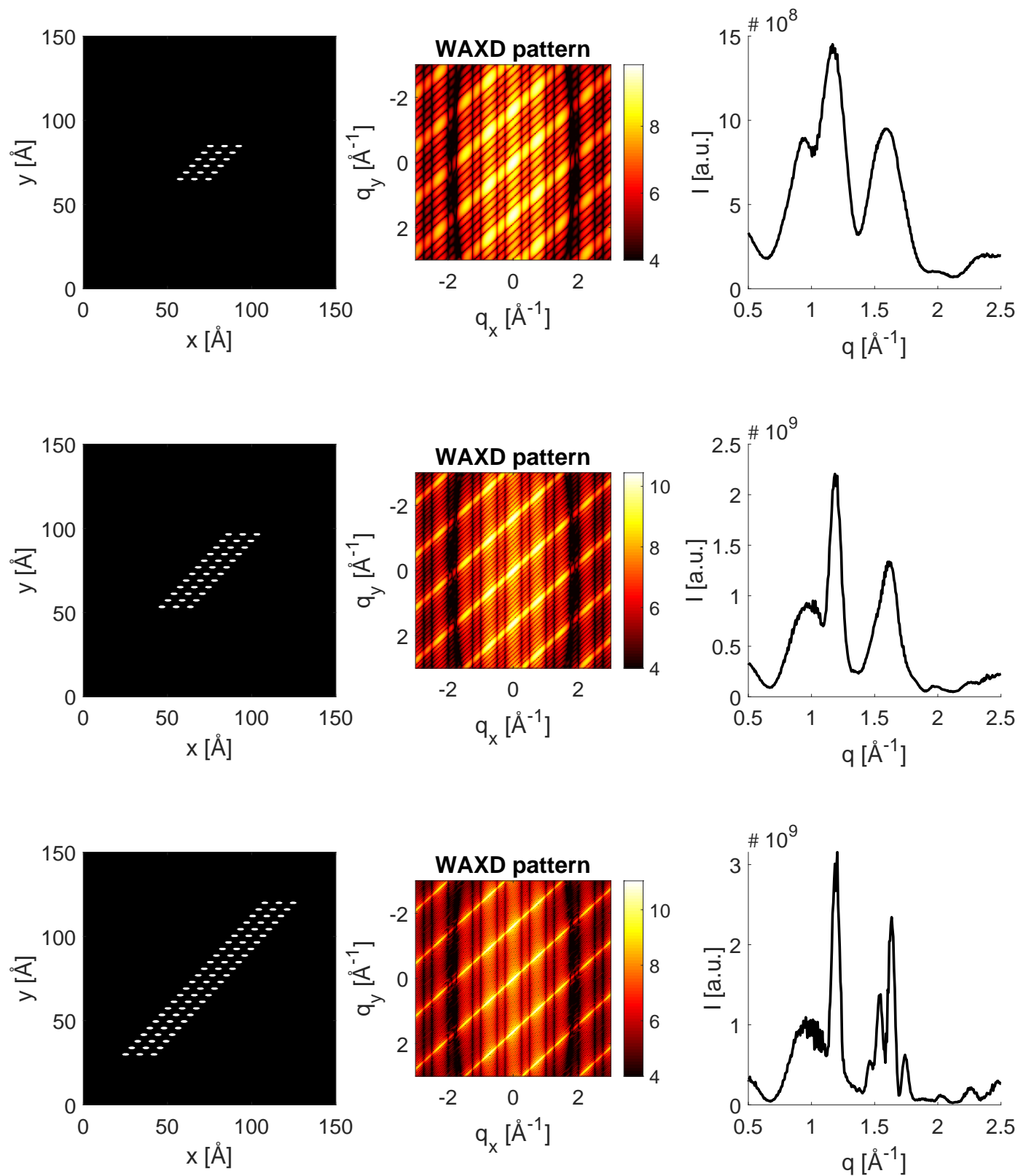
Model: Rh2

Aggregation direction: (010)

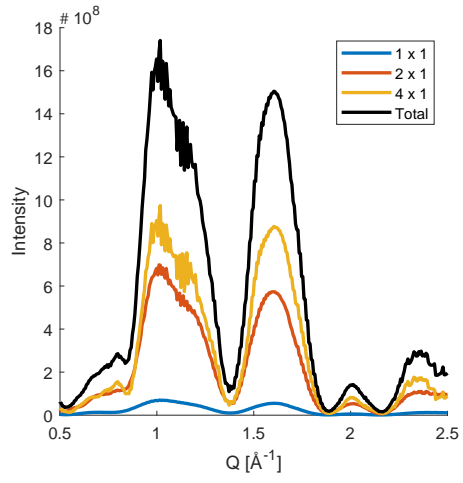

Sample \#1

Vol\% 1x1: 4.7

Vol\% $2 \times 1: 42.3$

Vol\% $4 \times 1: 53.0$
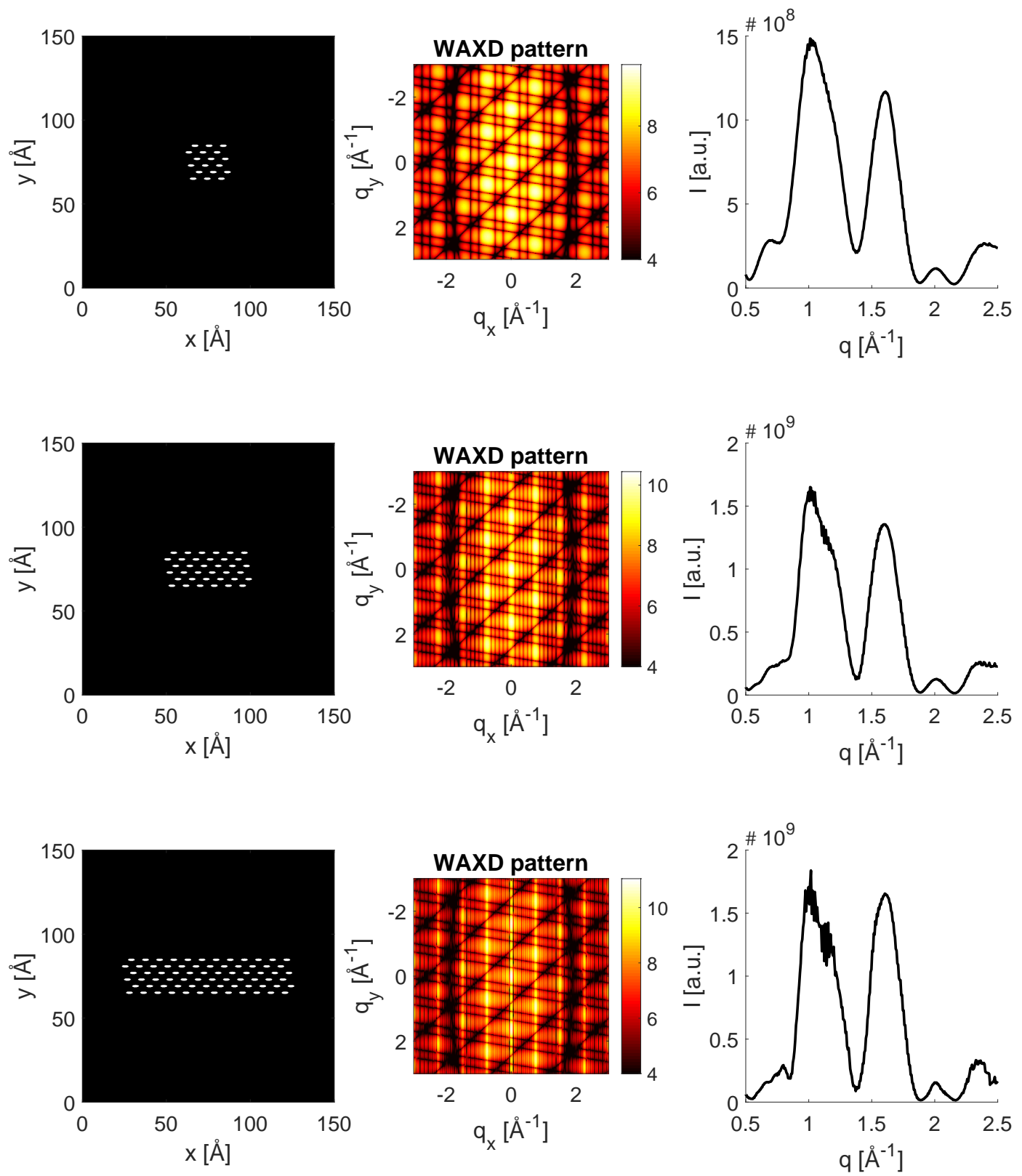
Model: Rh2

Aggregation direction: (200)

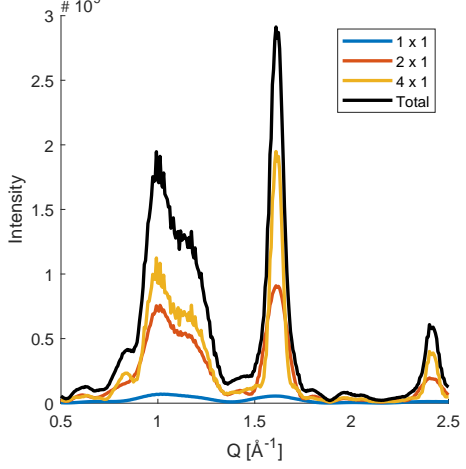

Sample \#1

Vol\% 1x1: 4.7

Vol\% 2x1: 42.3

Vol\% 4x1: 53.0
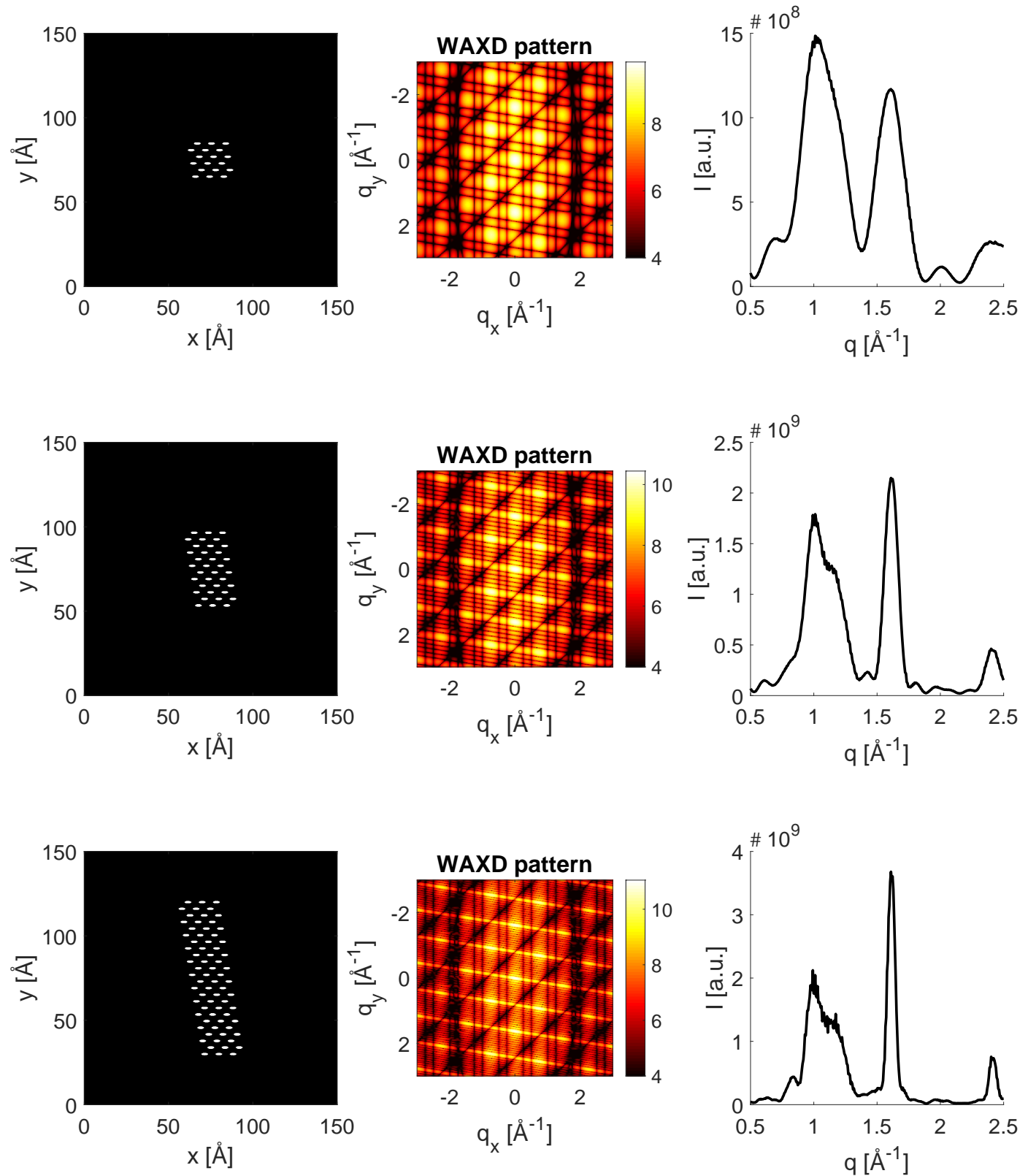

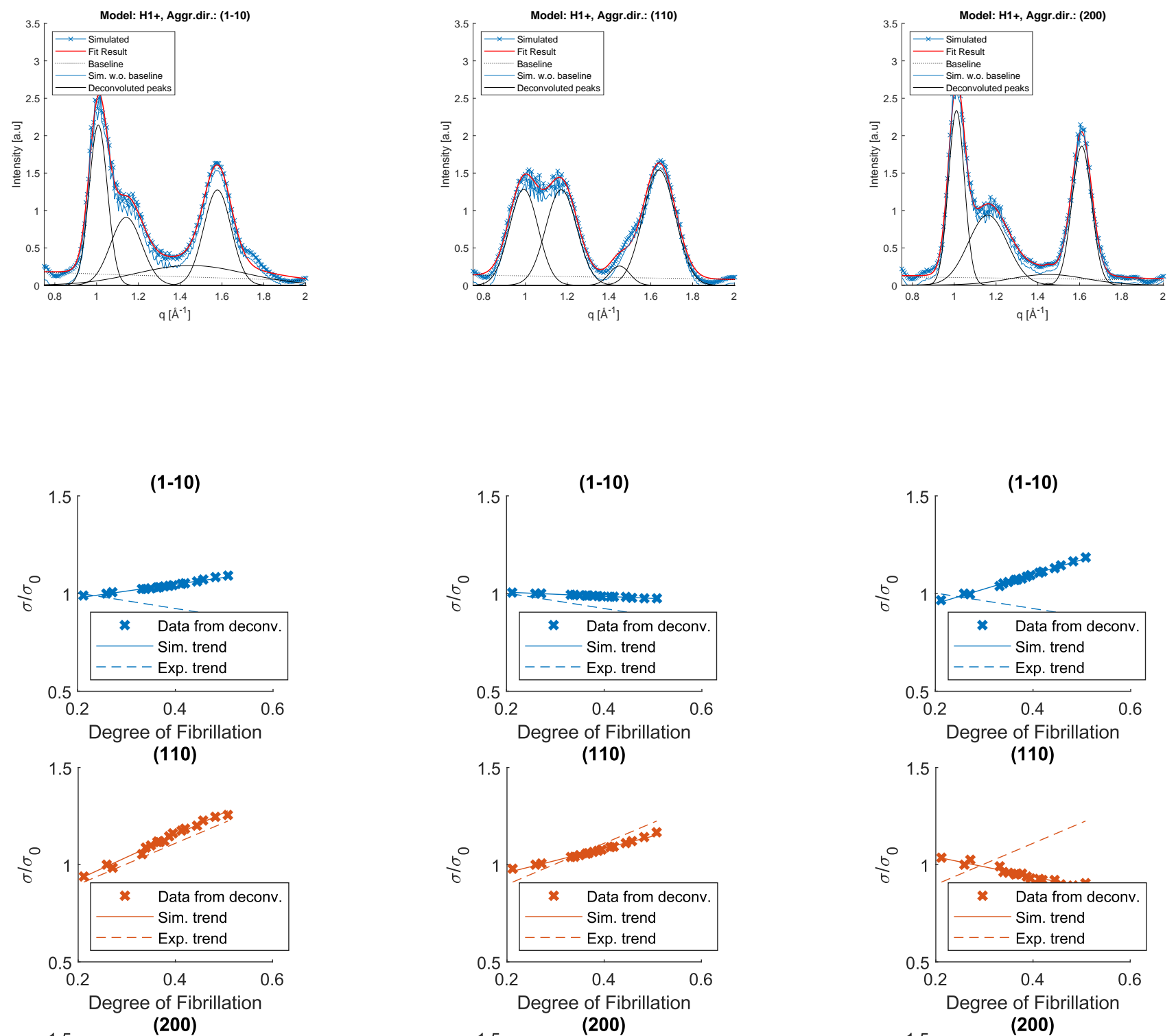

(110)
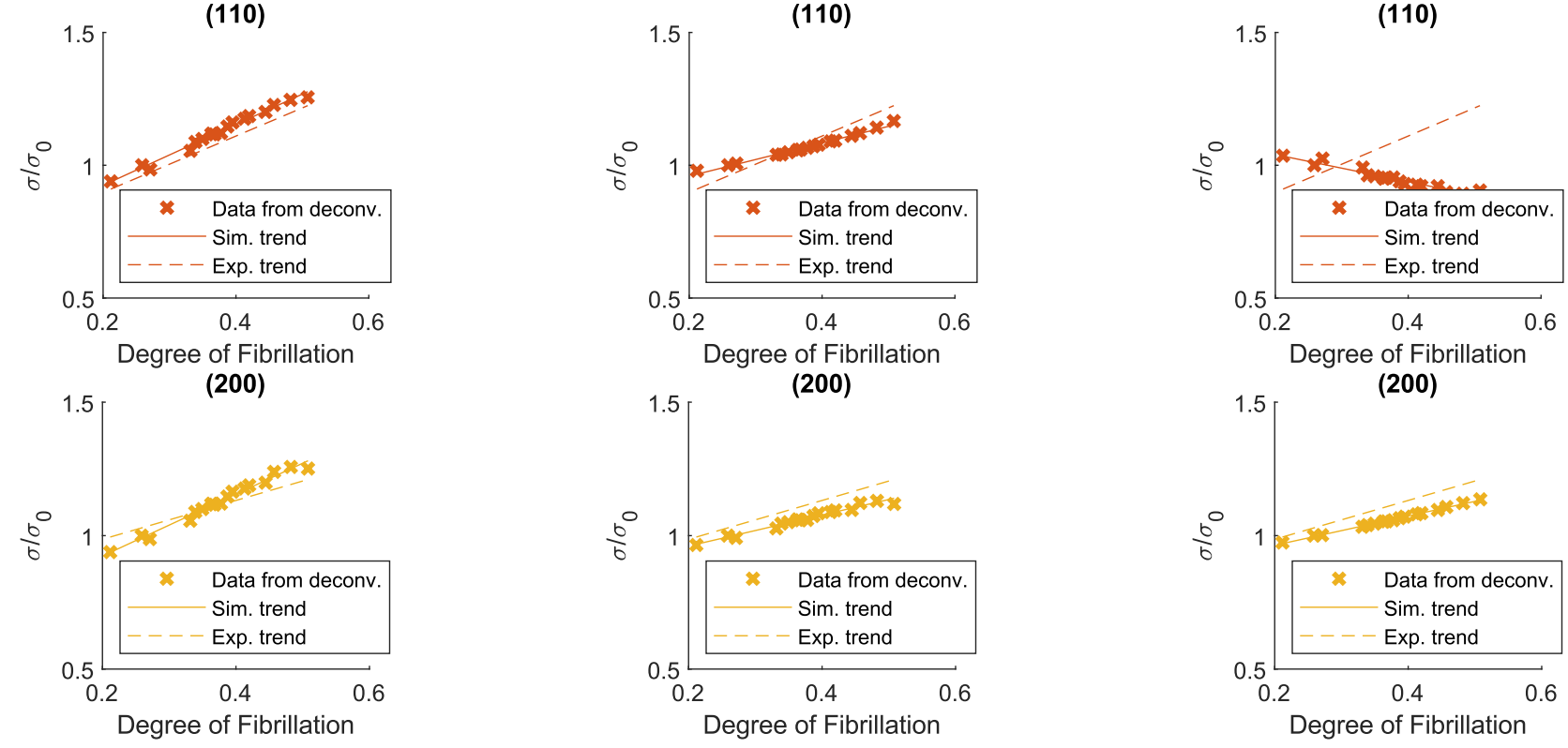

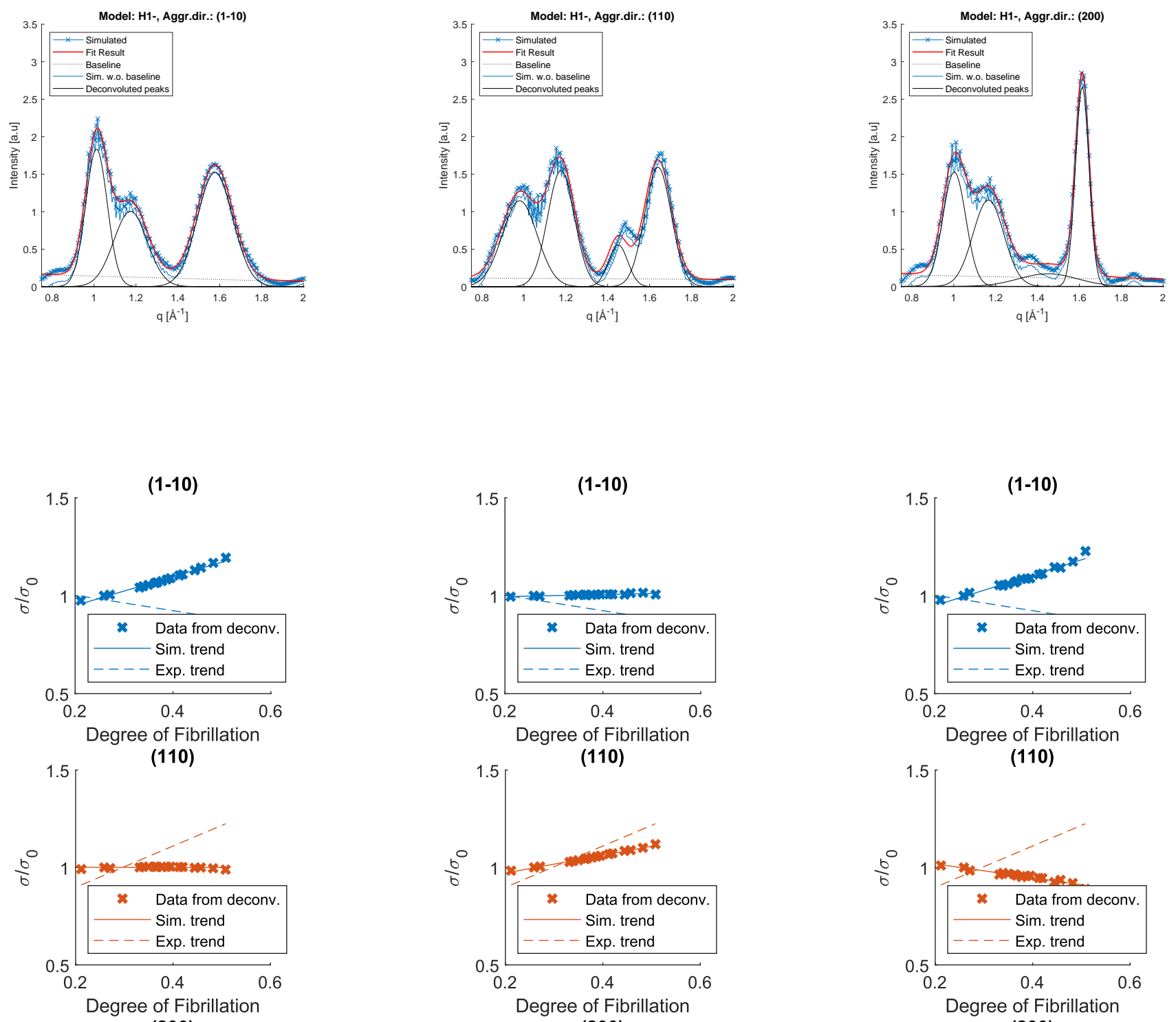

(110)
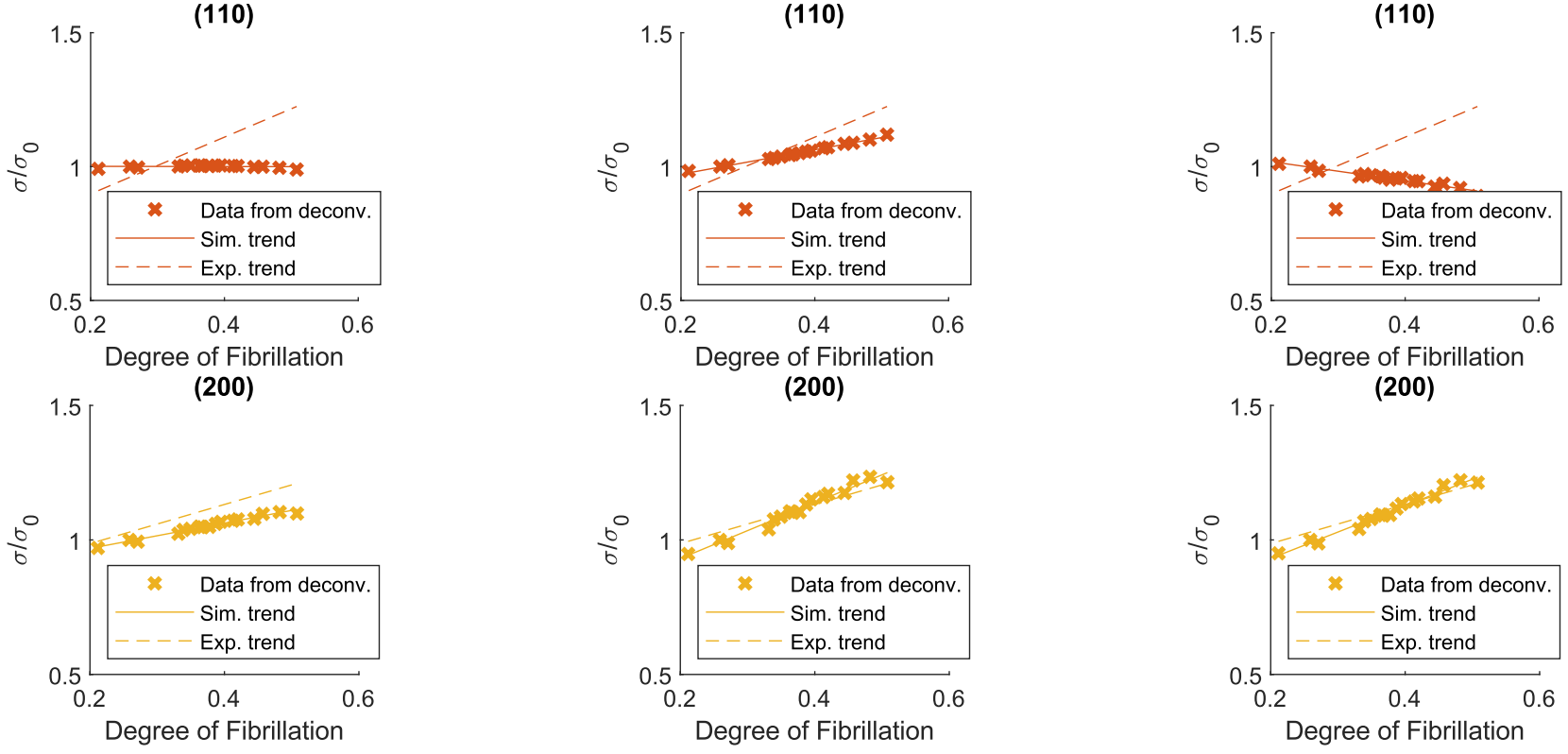

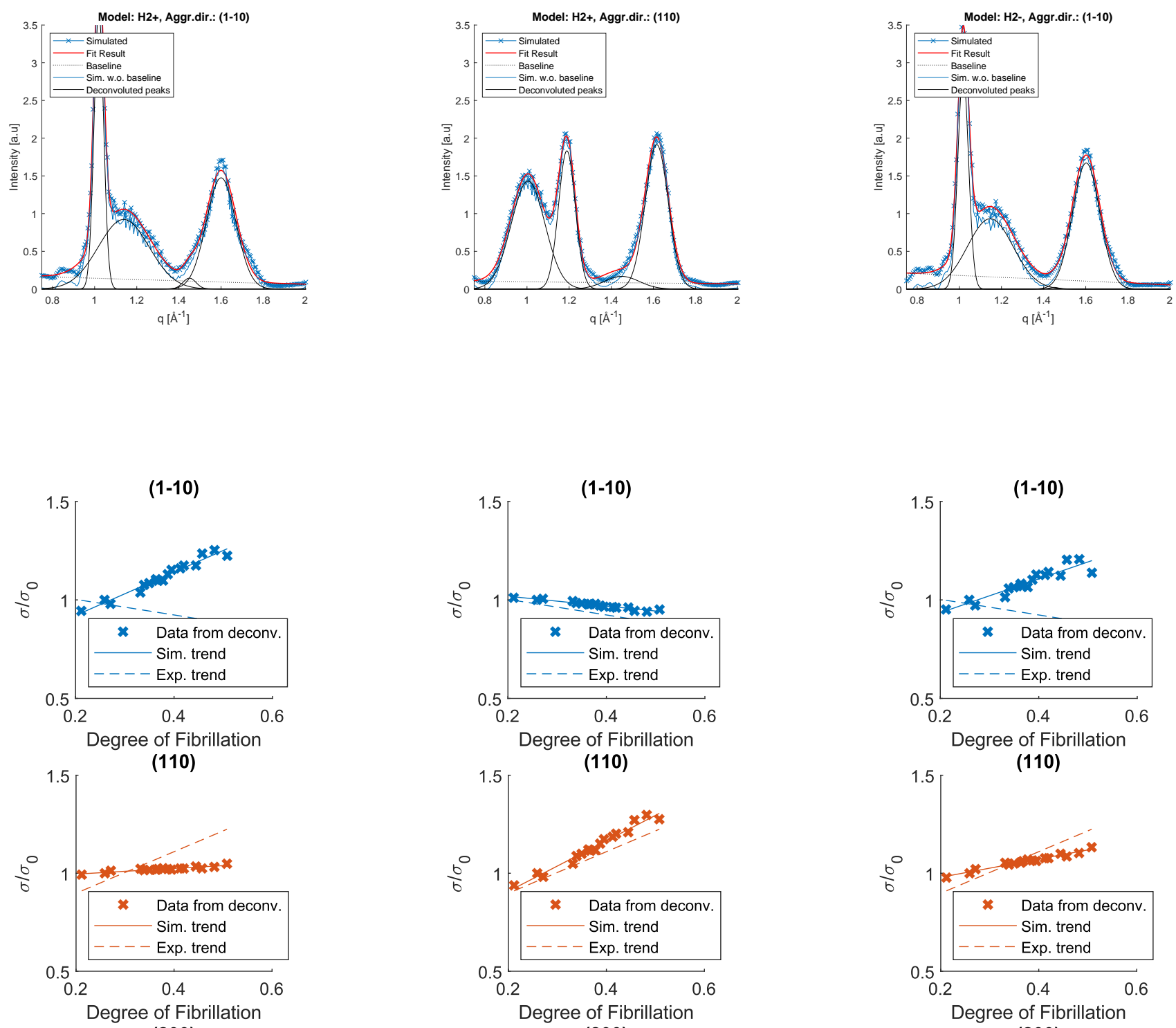

(110)

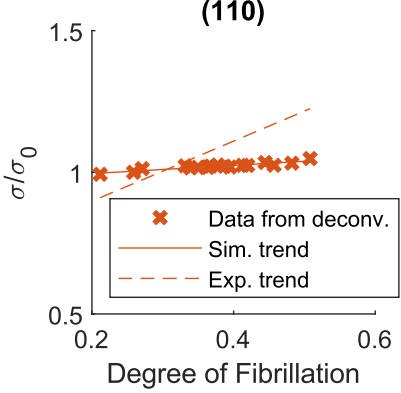

(200)
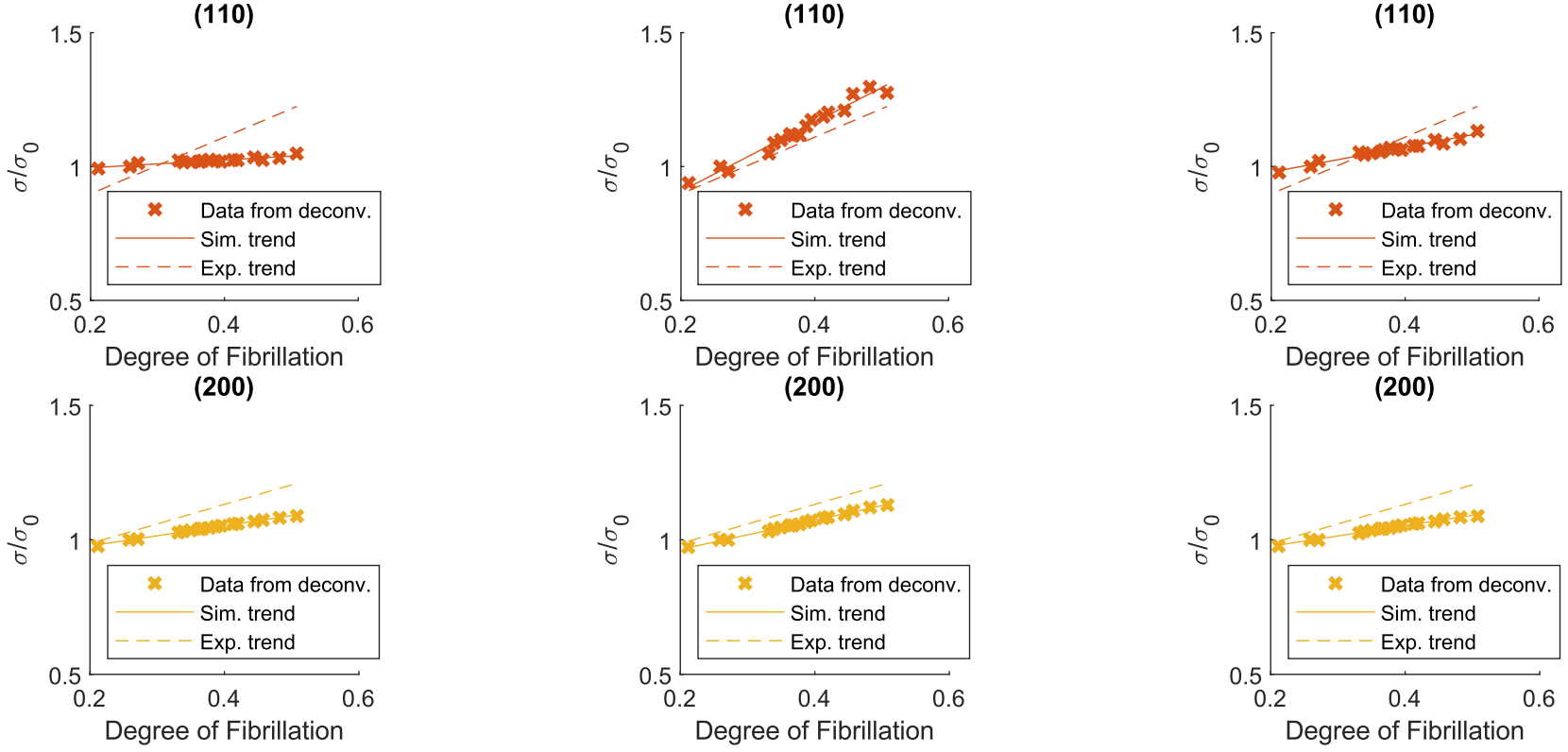

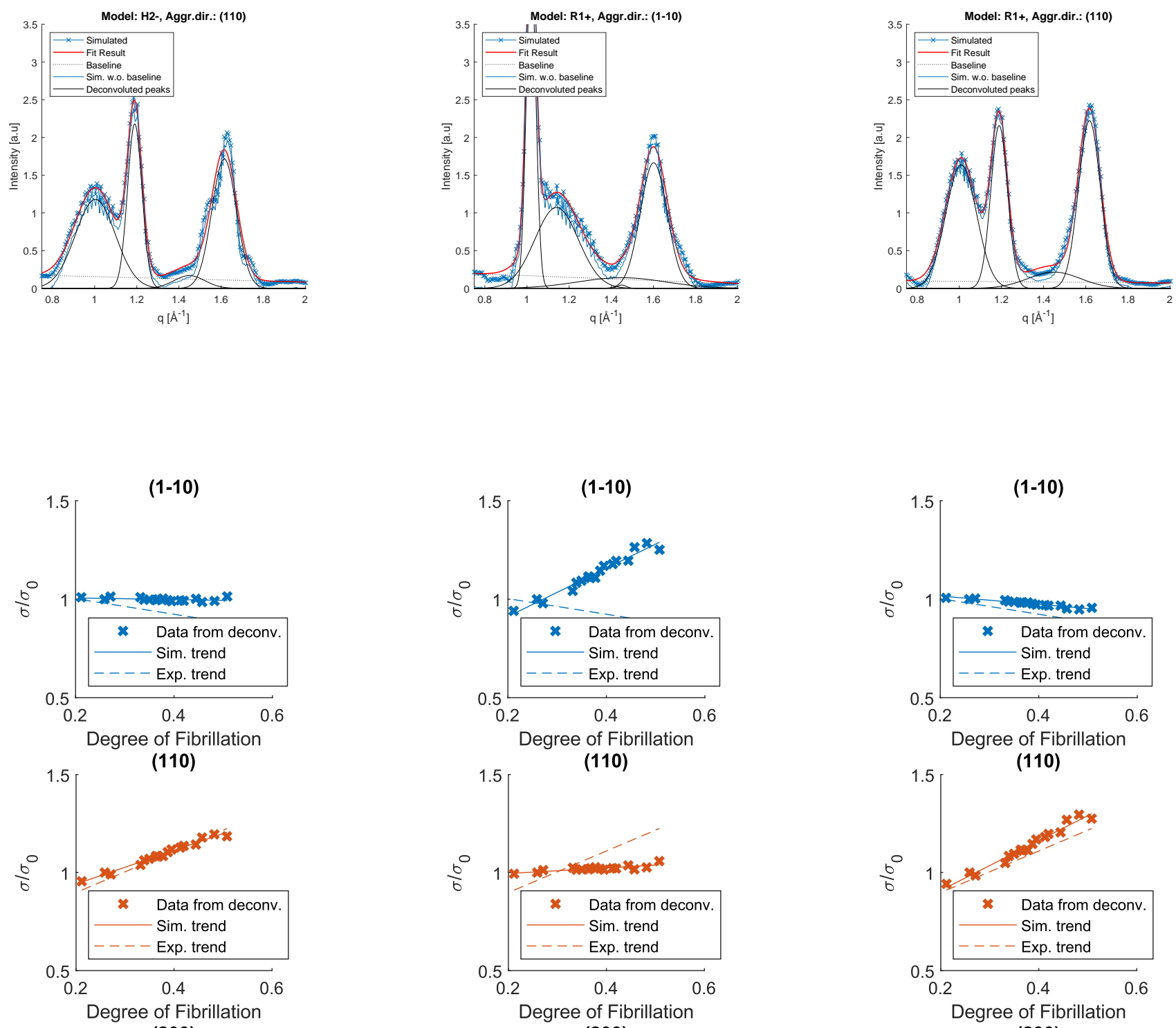

(110)

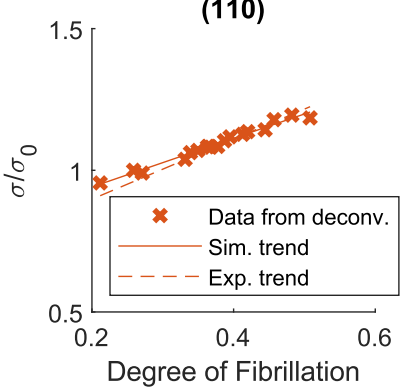

(200)
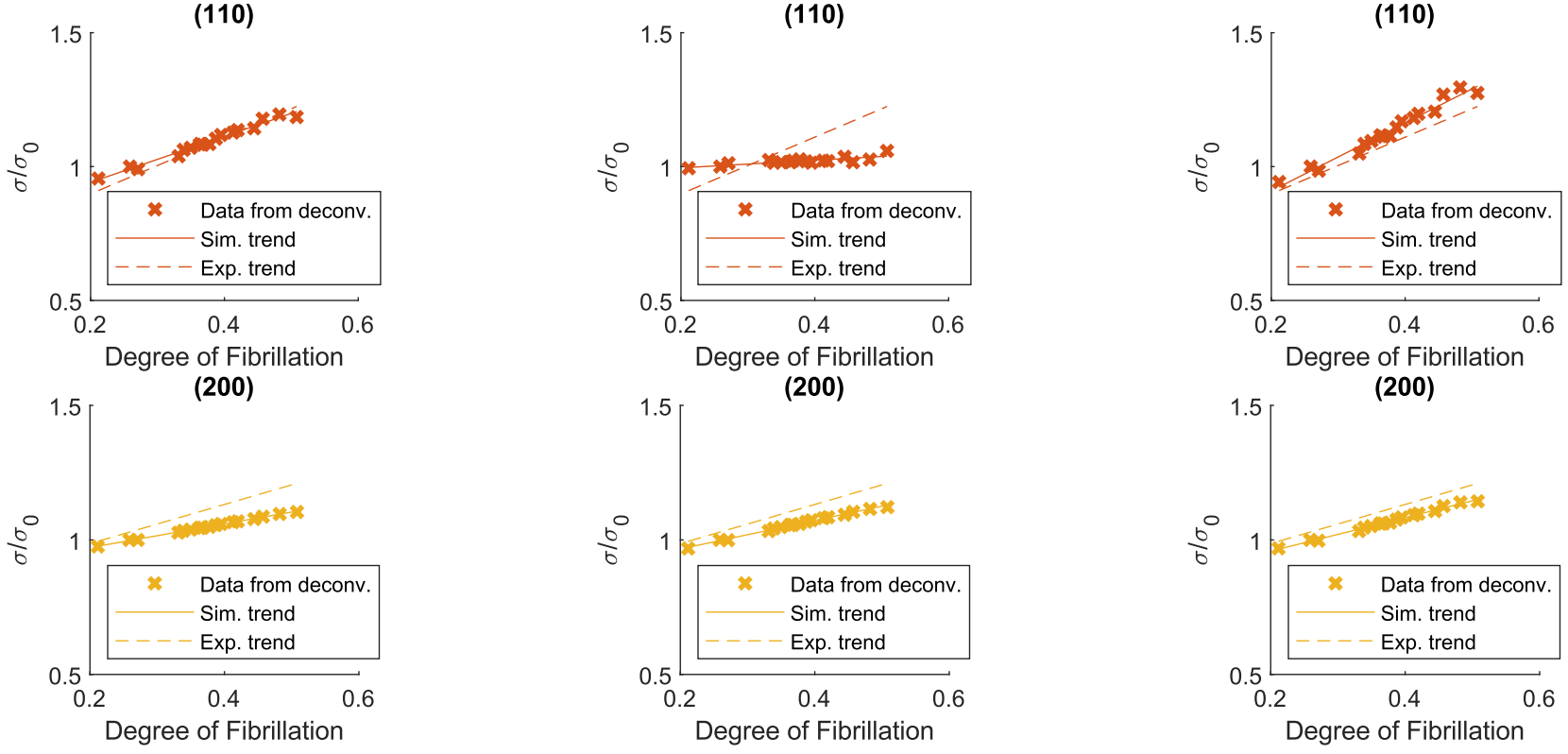

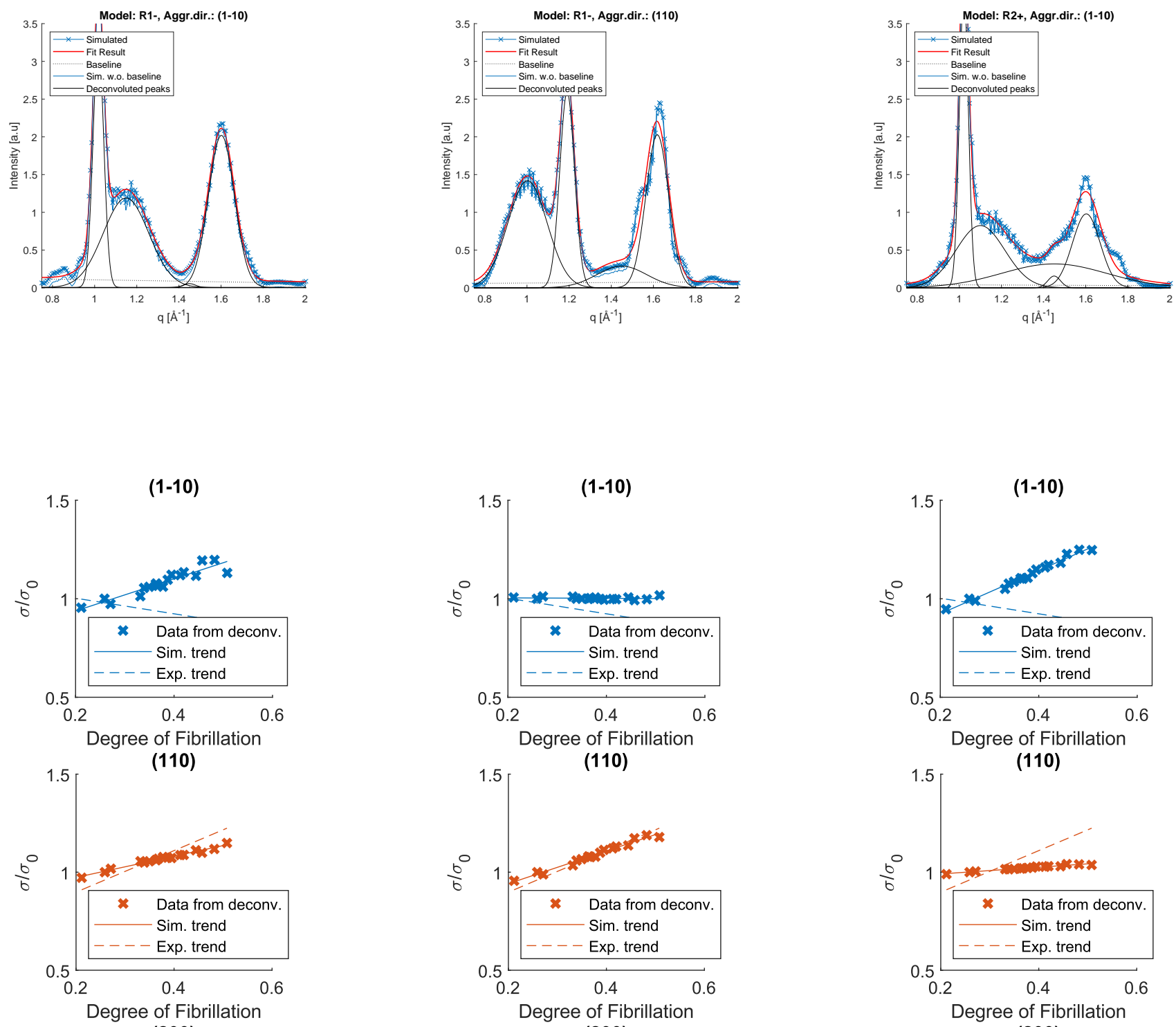

(110)
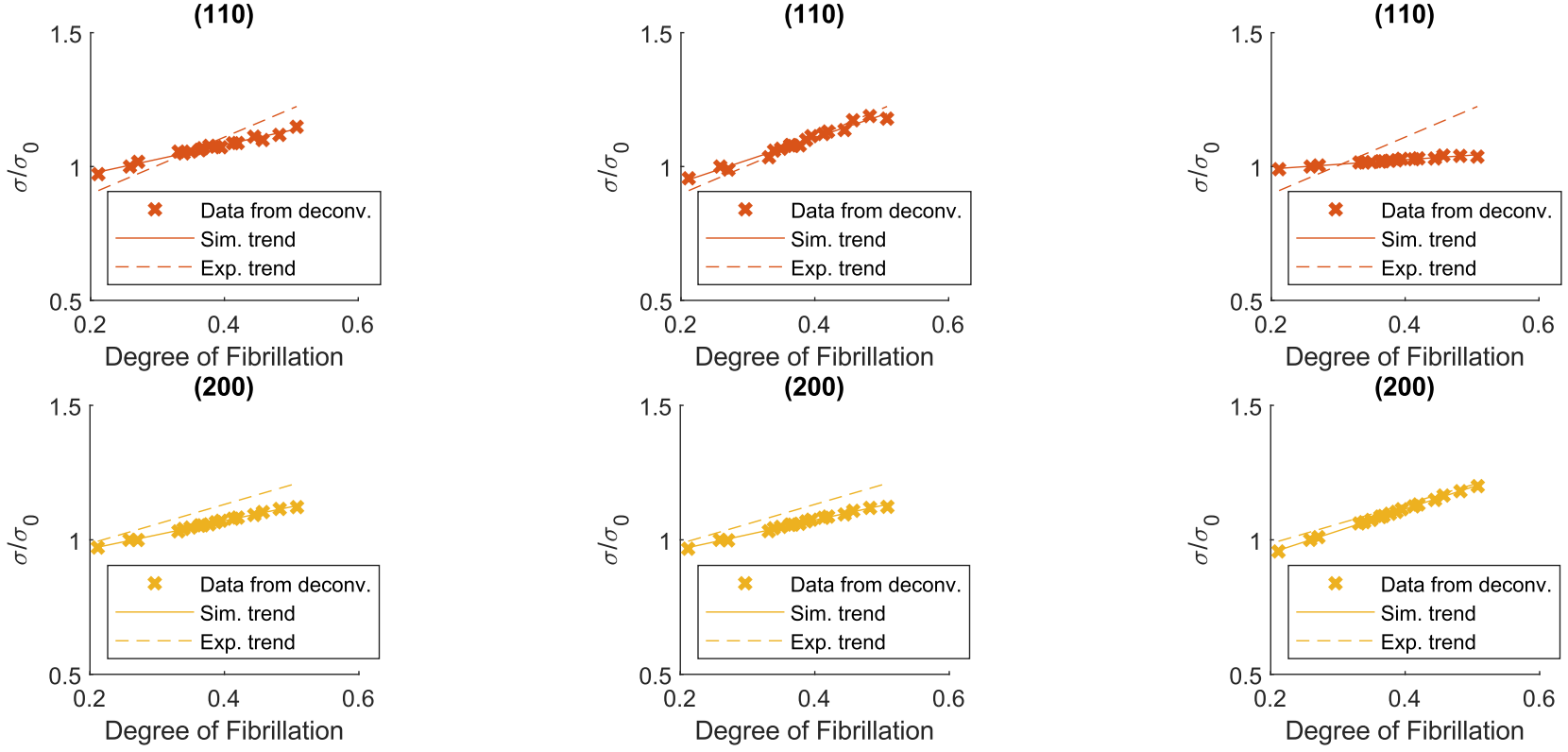

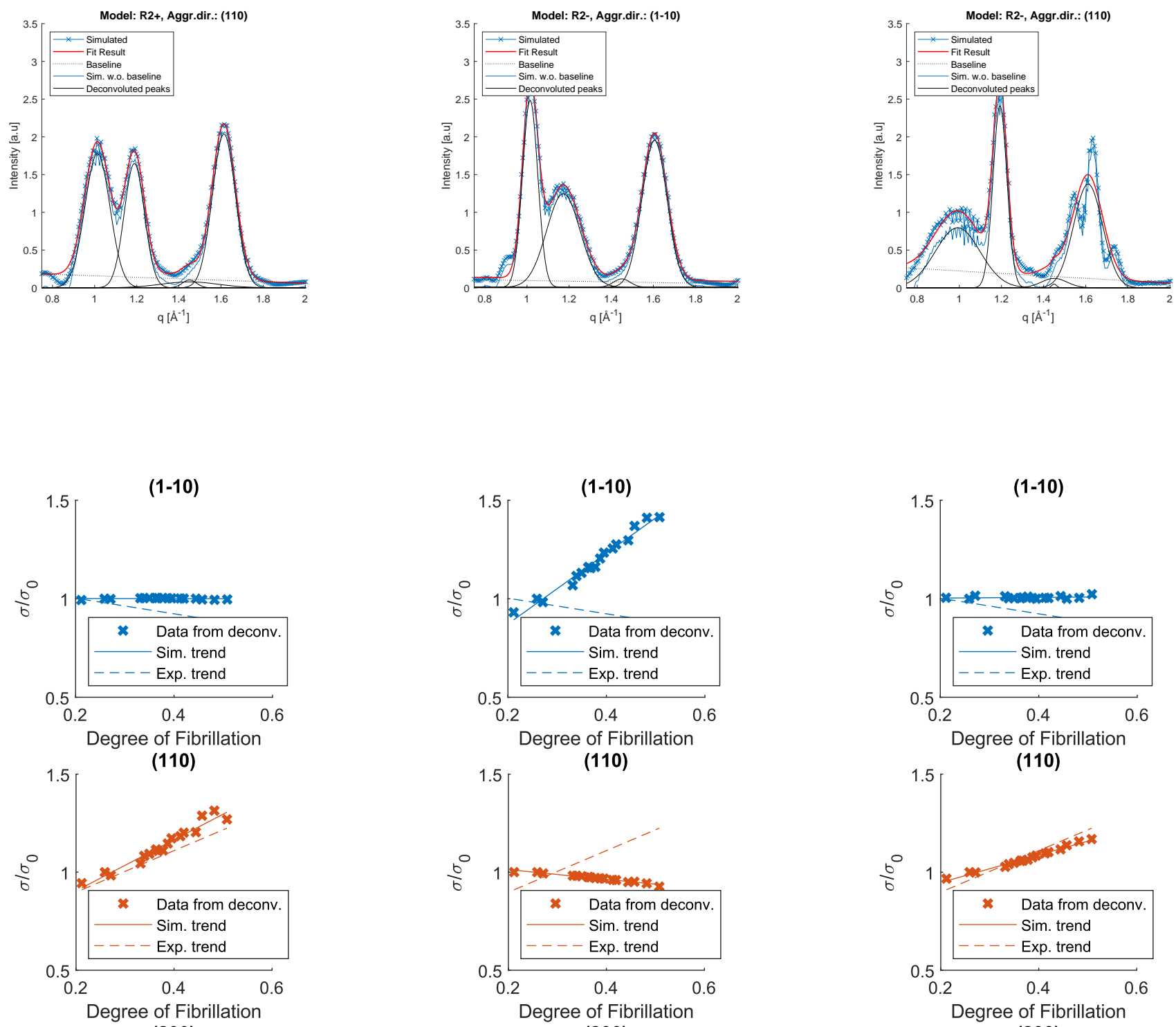

(110)
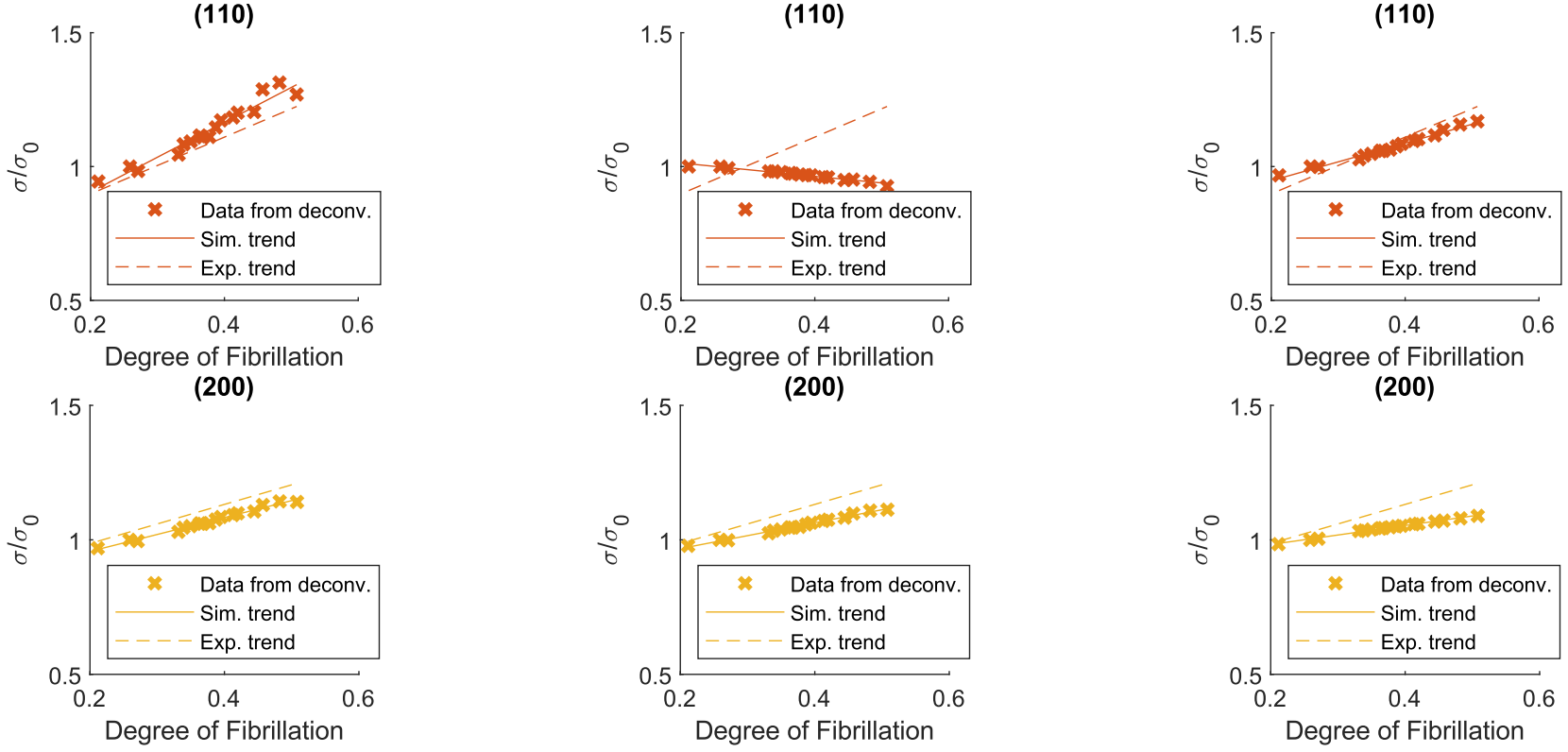

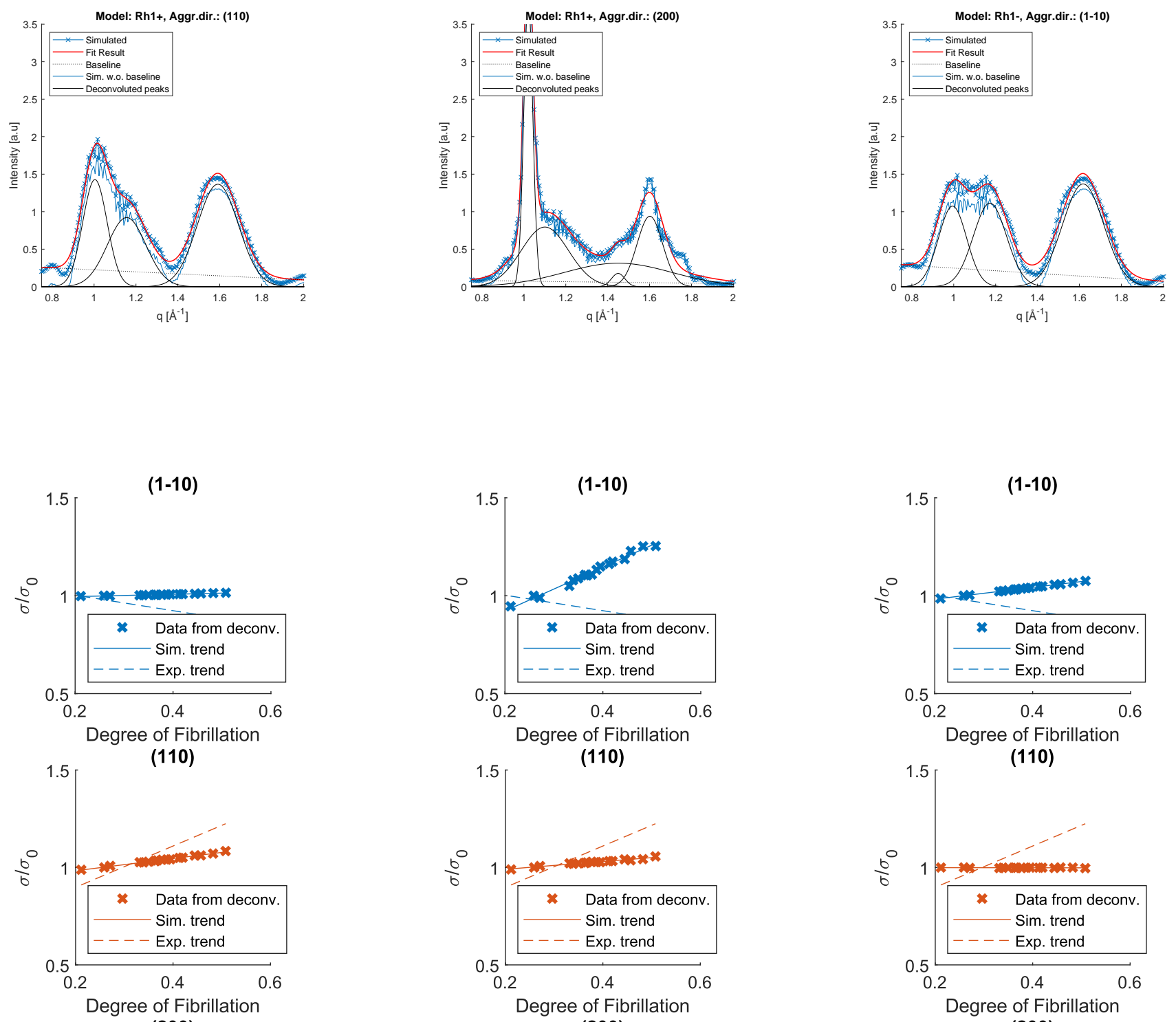

(110)
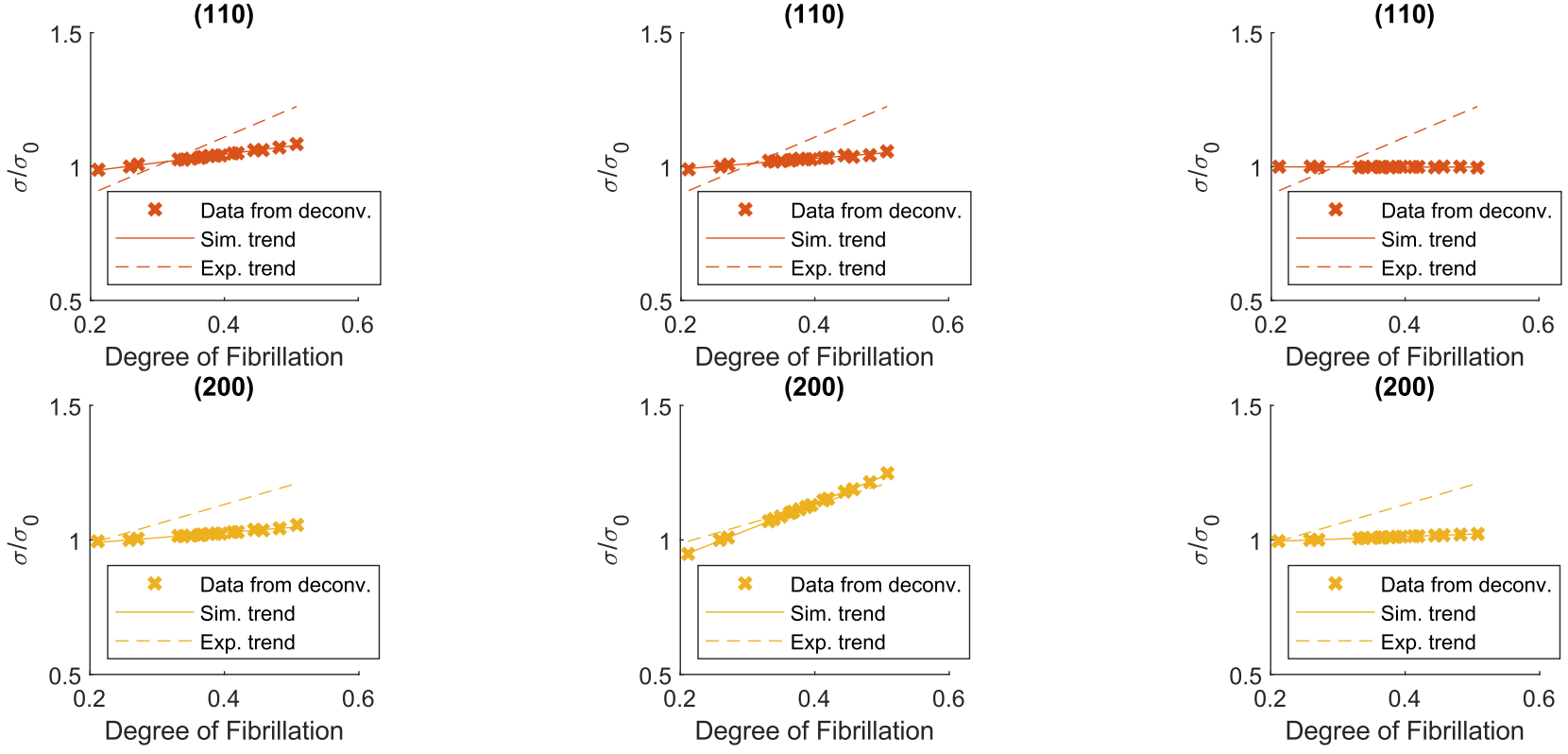

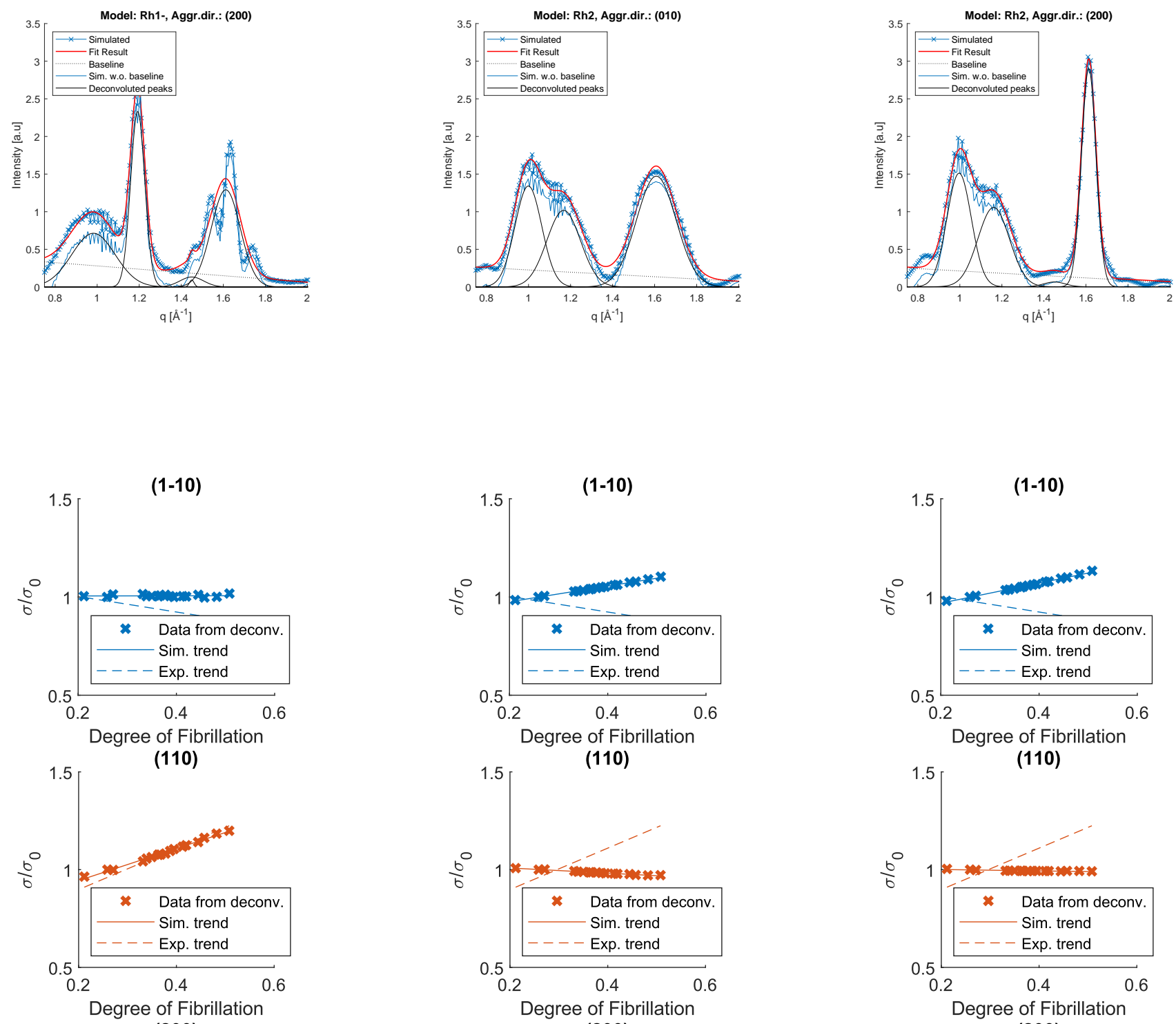

(110)
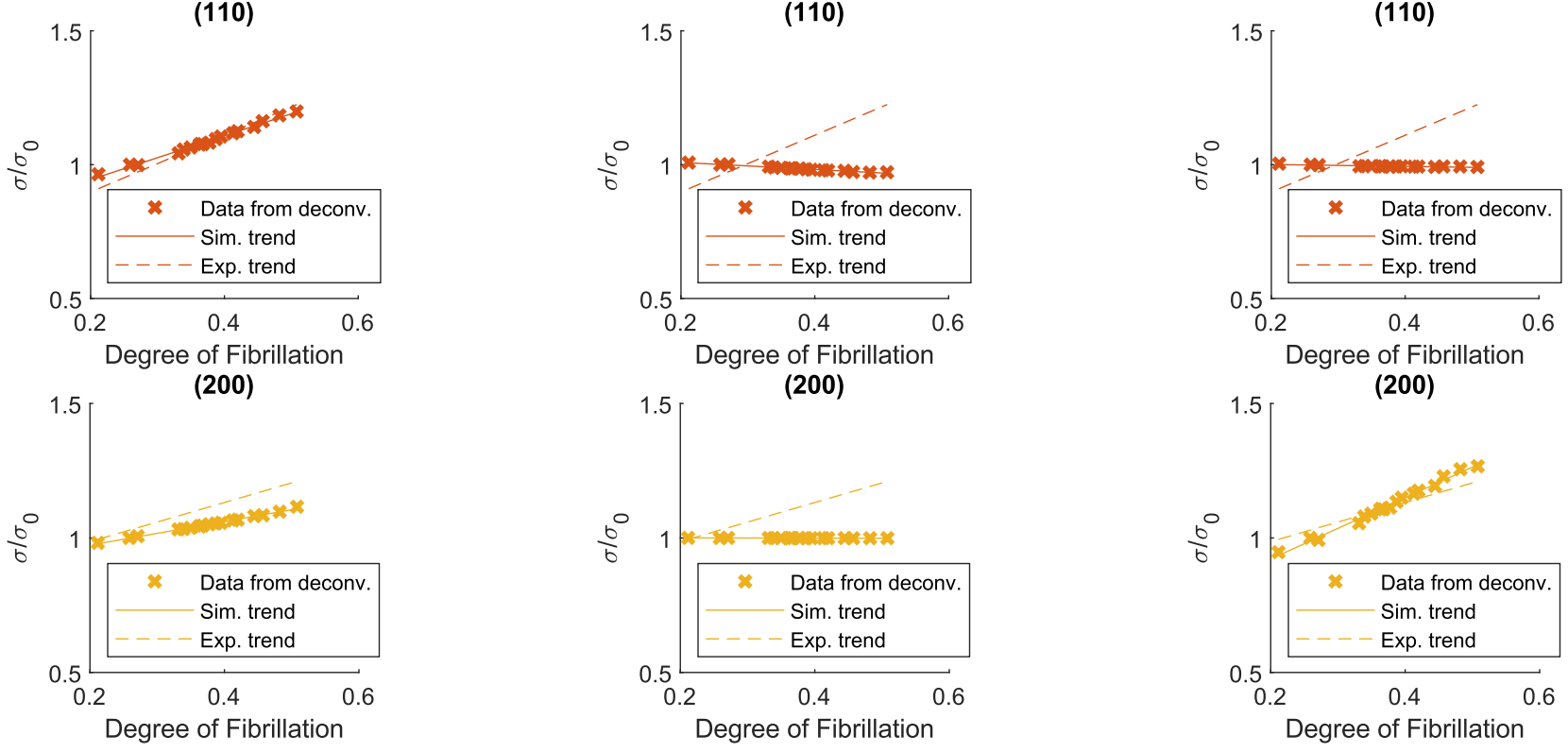


\section{WAXD simulations: Results}

Form factor of a CNF chain with semi-axes

$3 \AA ̊ 00.5 \AA$ 
Model: H1+

Aggregation direction: (1-10)

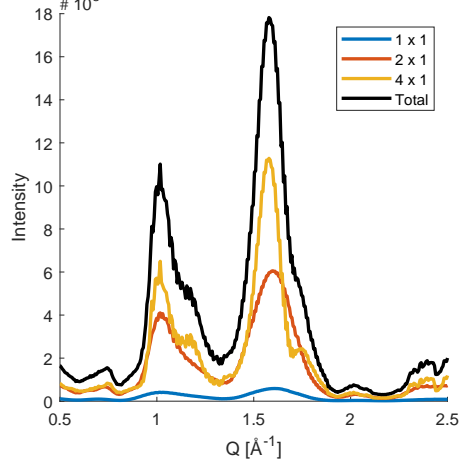

Sample \#1

Vol\% 1x1: 4.7

Vol\% 2x1: 42.3

Vol\% 4x1: 53.0
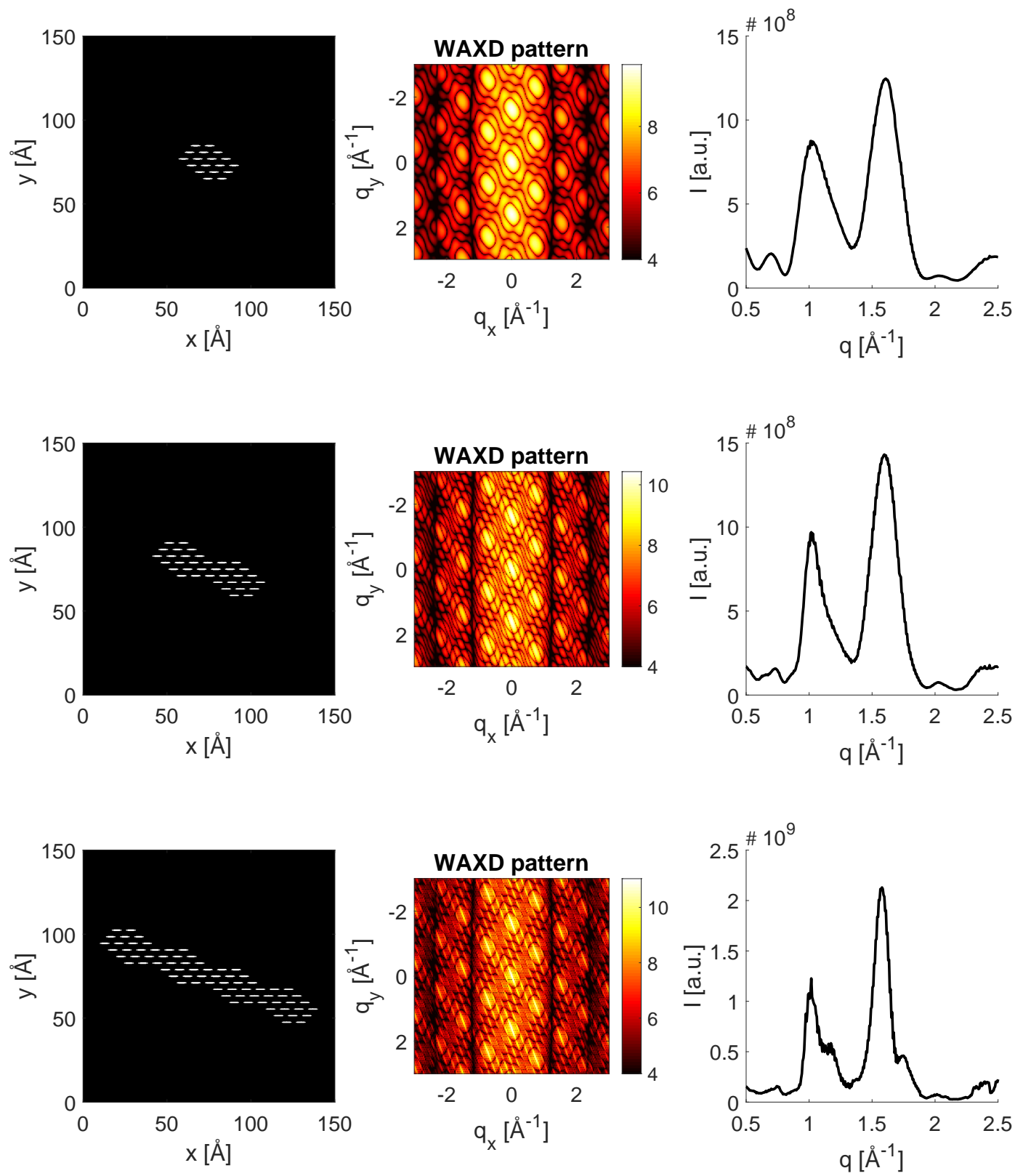
Model: H1+

Aggregation direction: (110)

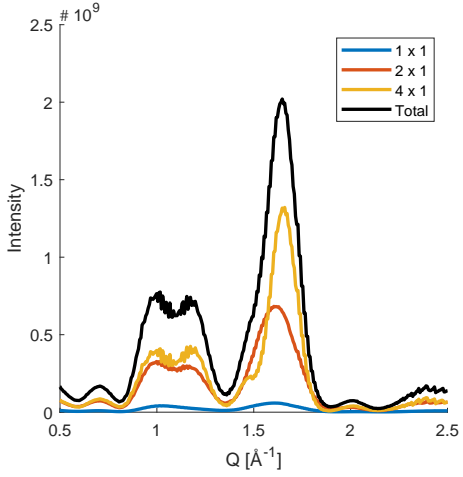

Sample \#1

Vol\% 1x1: 4.7

Vol\% 2x1: 42.3

Vol\% $4 \times 1: 53.0$
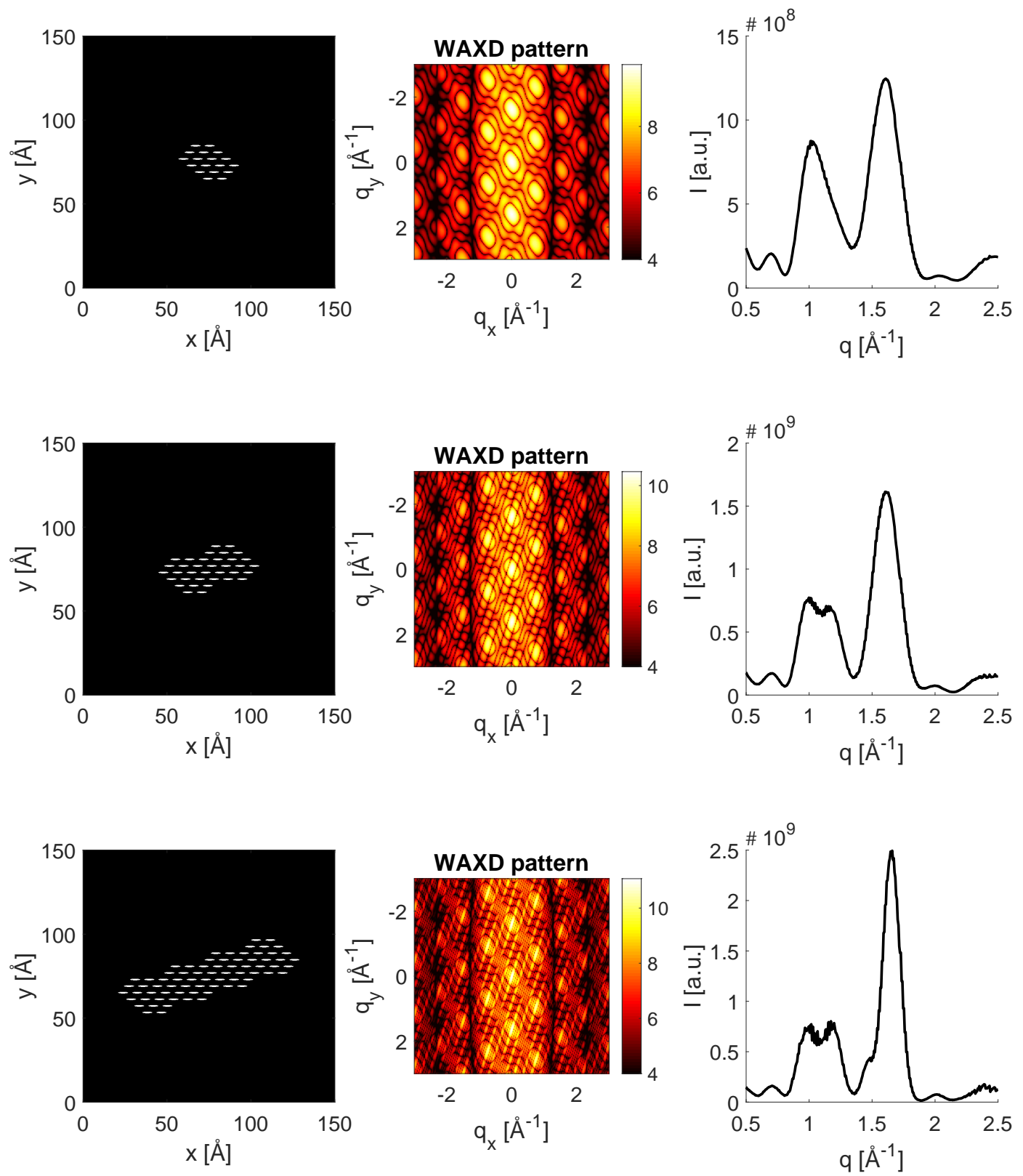
Model: H1+

Aggregation direction: (200)

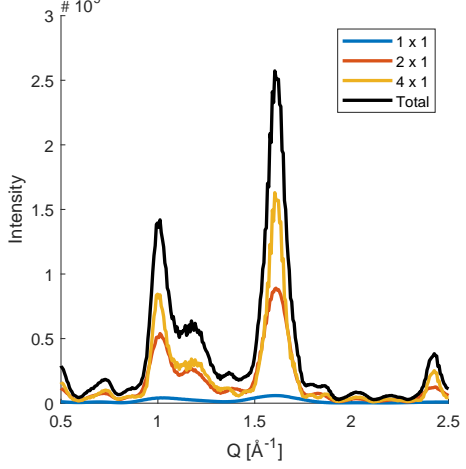

Sample \#1

Vol\% 1x1: 4.7

Vol\% $2 \times 1: 42.3$

Vol\% $4 \times 1: 53.0$
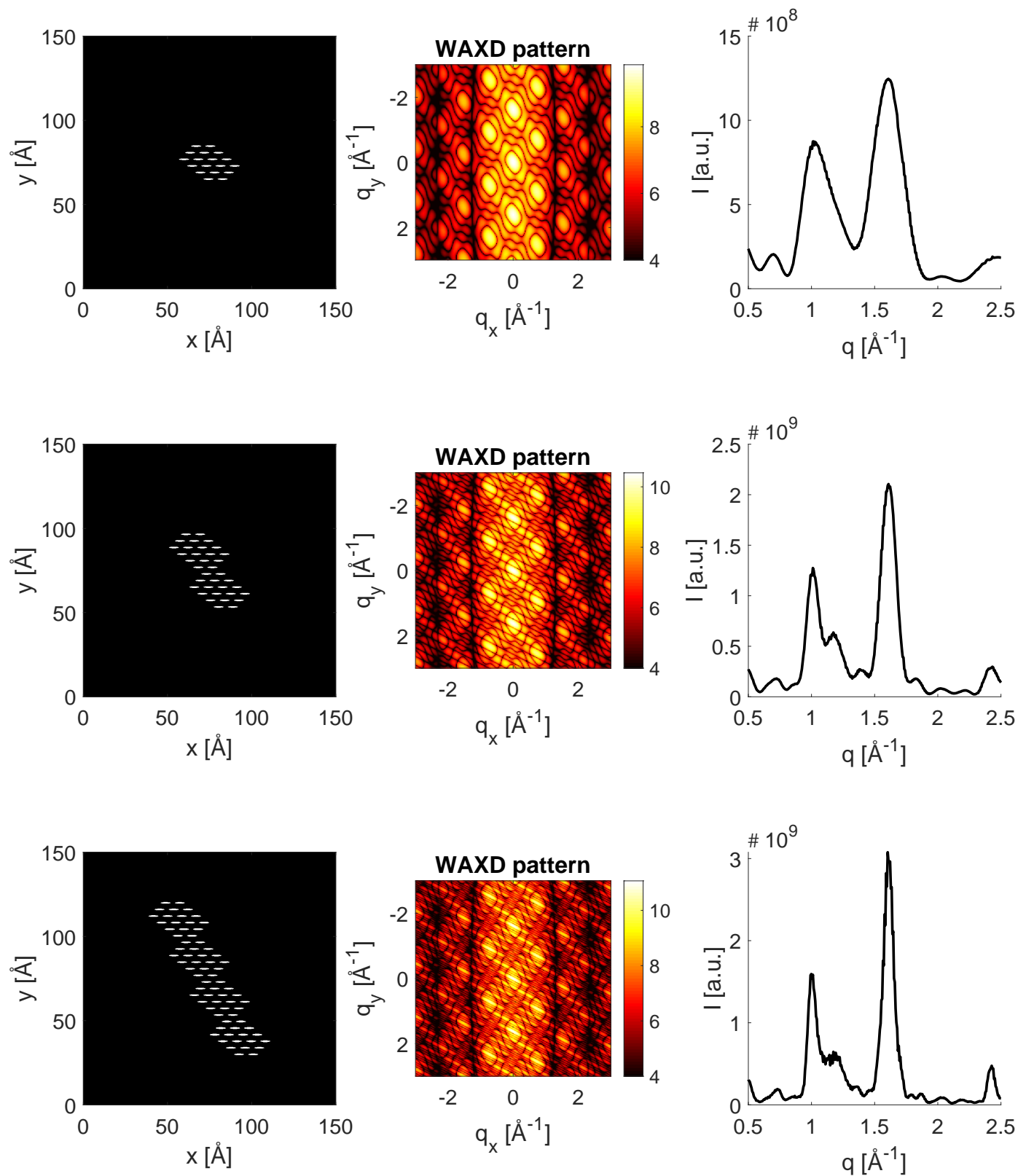
Model: H1-

Aggregation direction: (1-10)

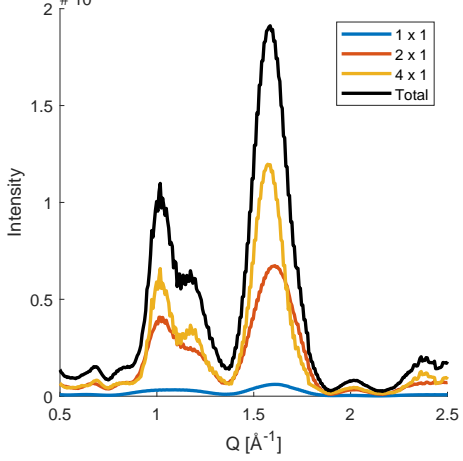

Sample \#1

Vol\% 1x1: 4.7

Vol\% 2x1: 42.3

Vol\% 4x1: 53.0
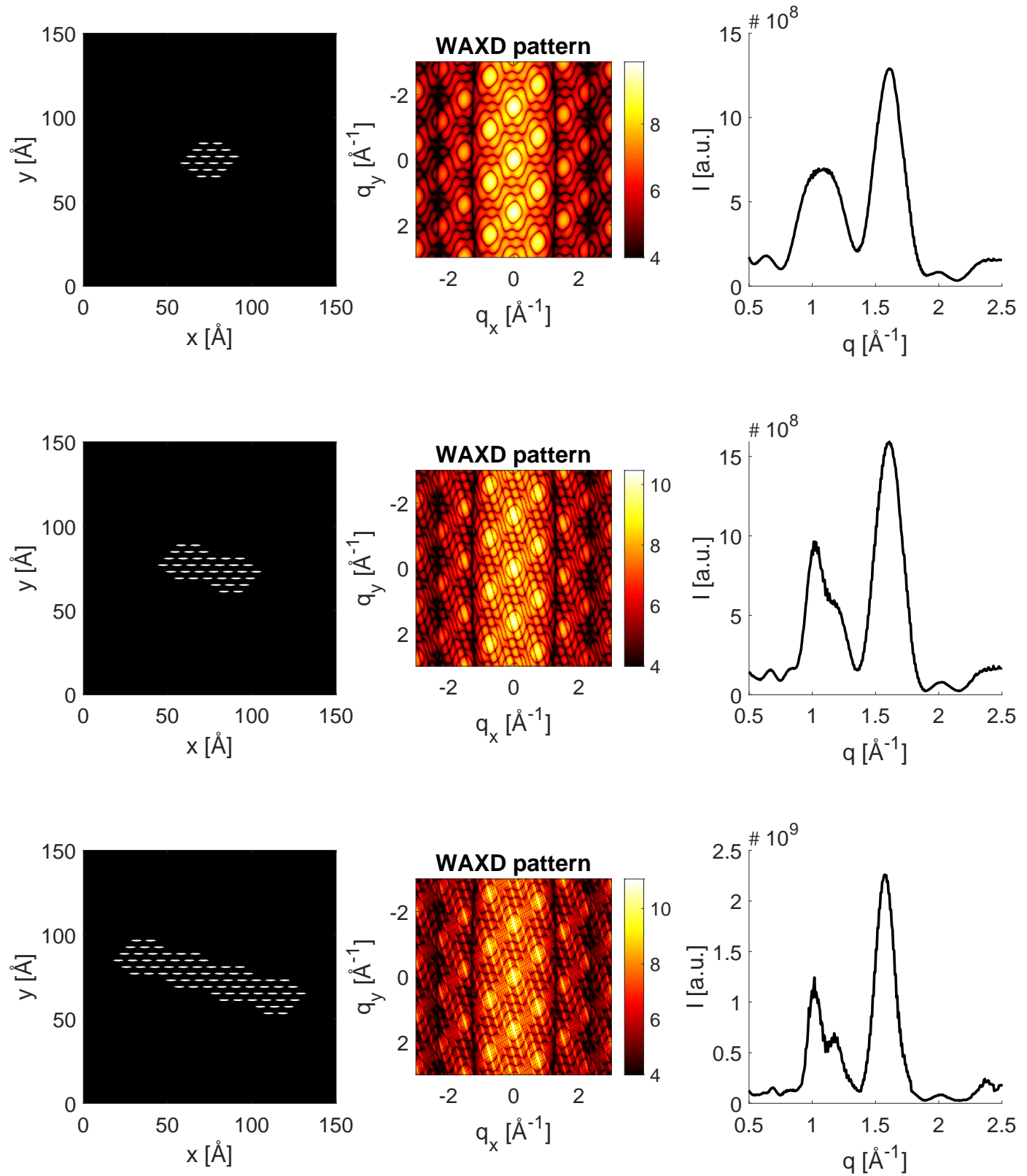
Aggregation direction: (110)

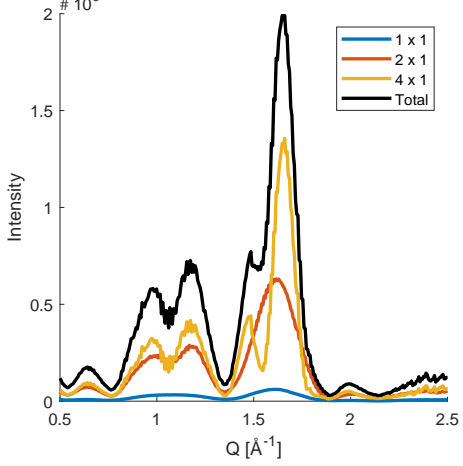

Sample \#1

Vol\% 1x1: 4.7

Vol\% 2x1: 42.3

Vol\% 4x1: 53.0
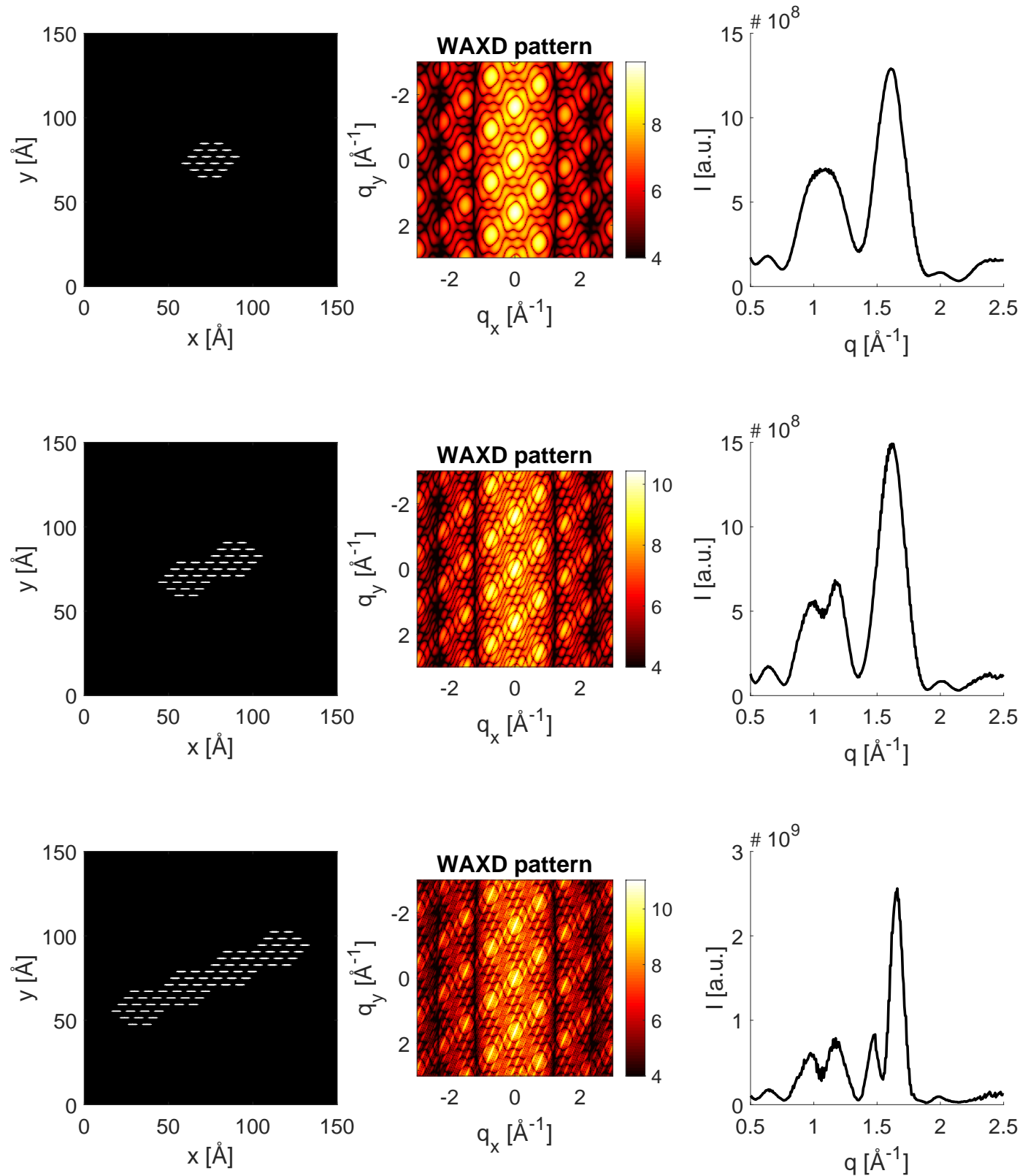
Model: H1-

Aggregation direction: (200)

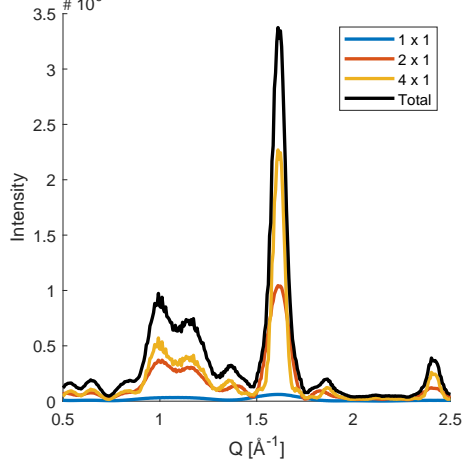

Sample \#1

Vol\% 1x1: 4.7

Vol\% $2 \times 1: 42.3$

Vol\% $4 \times 1: 53.0$
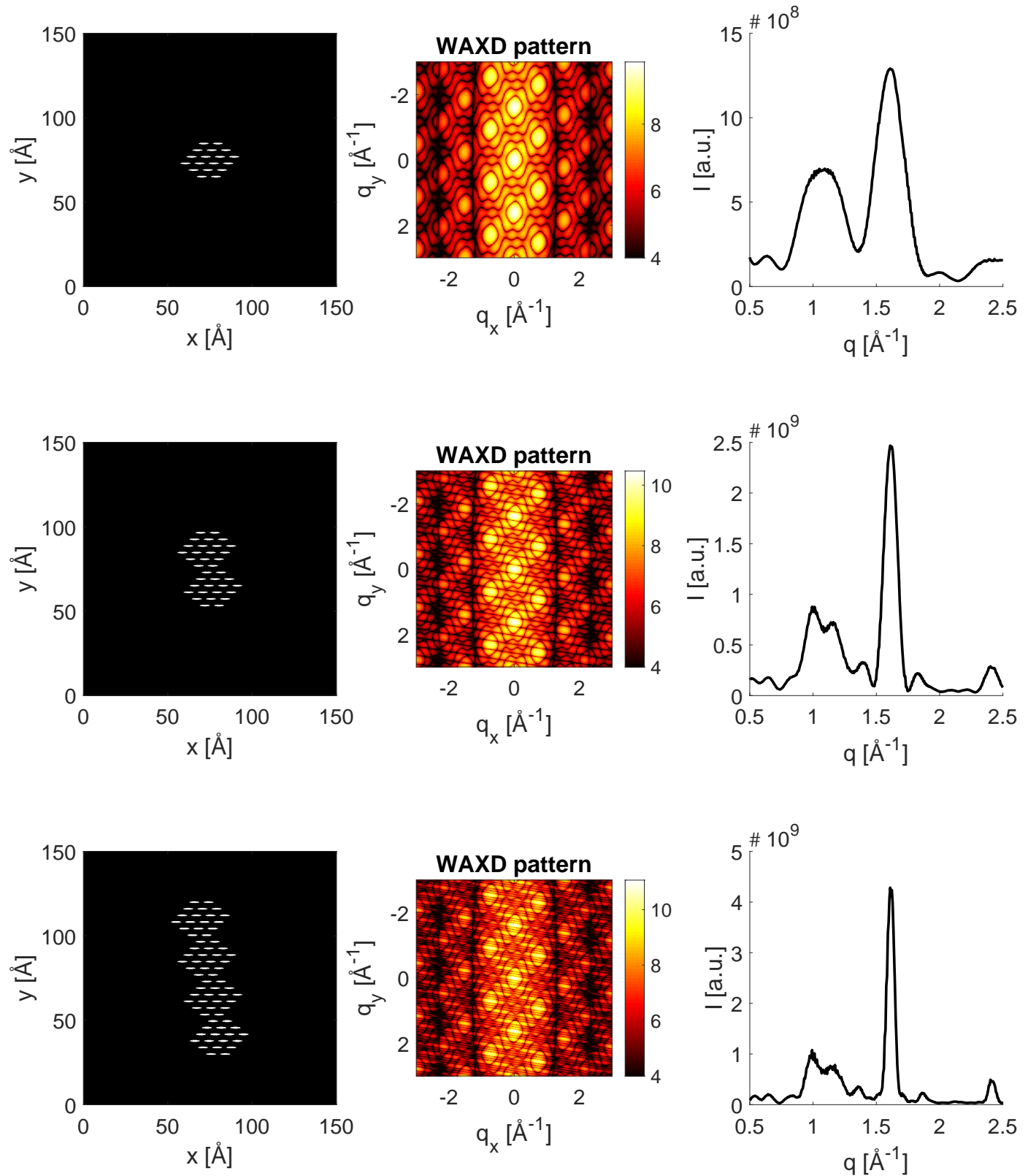
Model: $\mathrm{H} 2+$

Aggregation direction: (1-10)

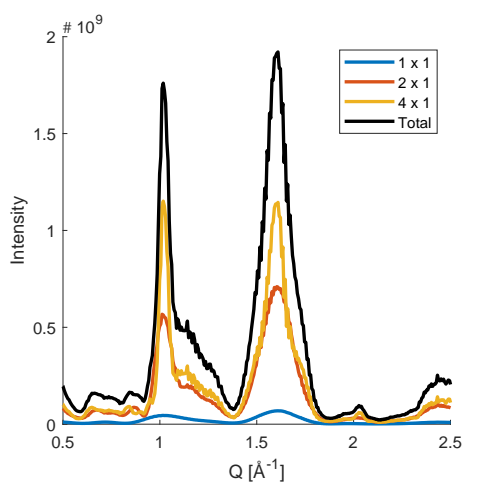

Sample \#1

Vol\% 1x1: 4.7

Vol\% $2 \times 1: 42.3$

Vol\% $4 \times 1: 53.0$
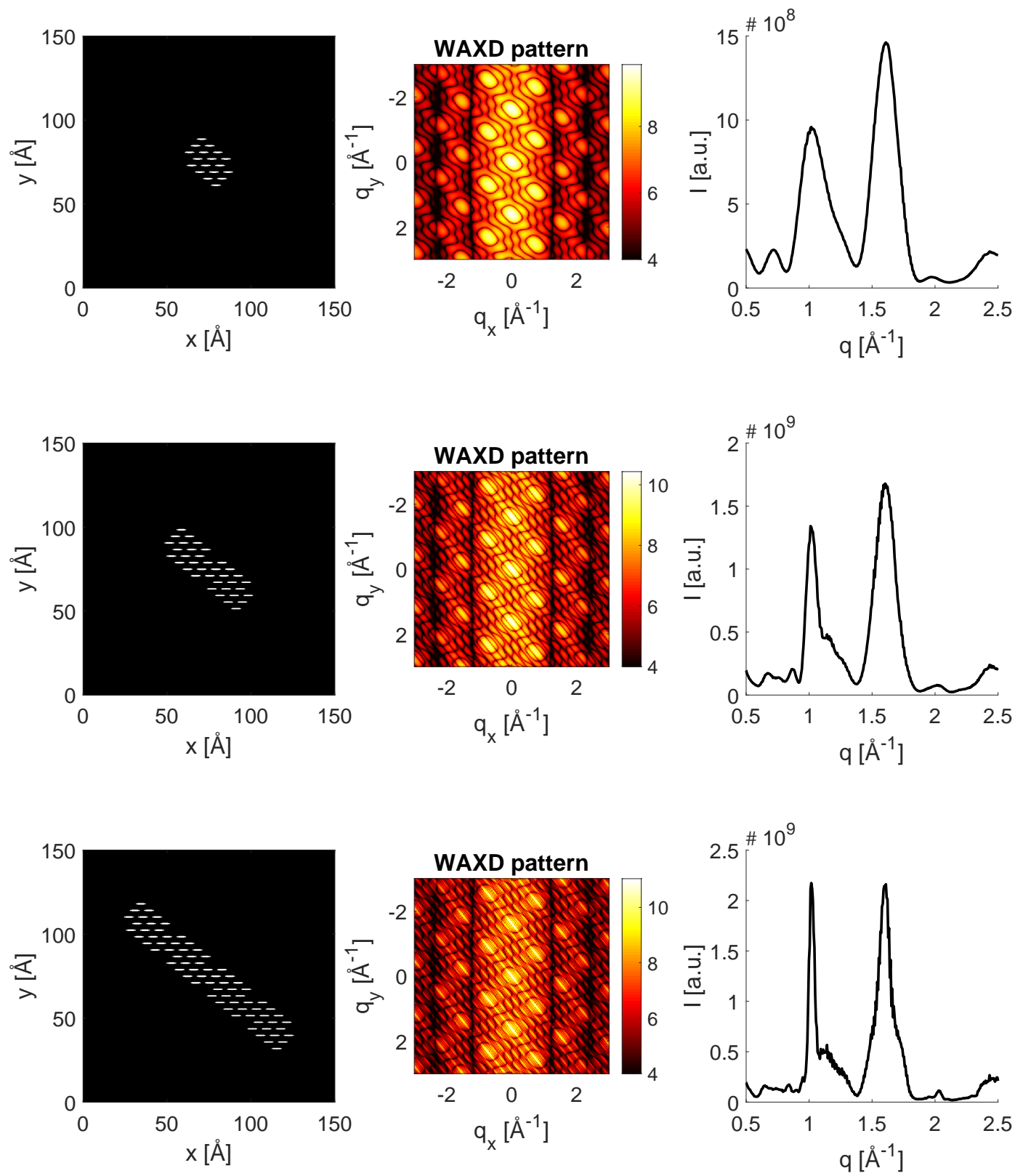
Model: $\mathrm{H} 2+$

Aggregation direction: (110)

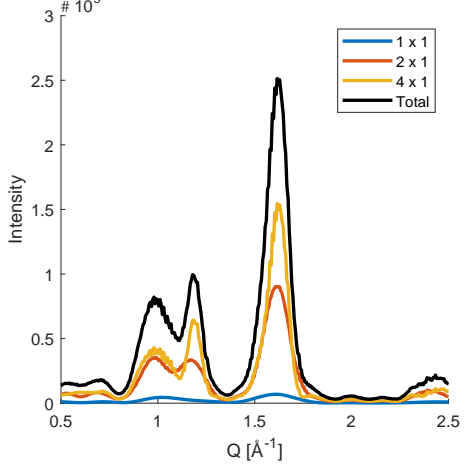

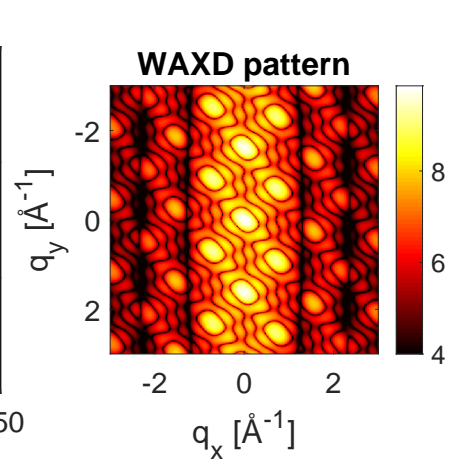

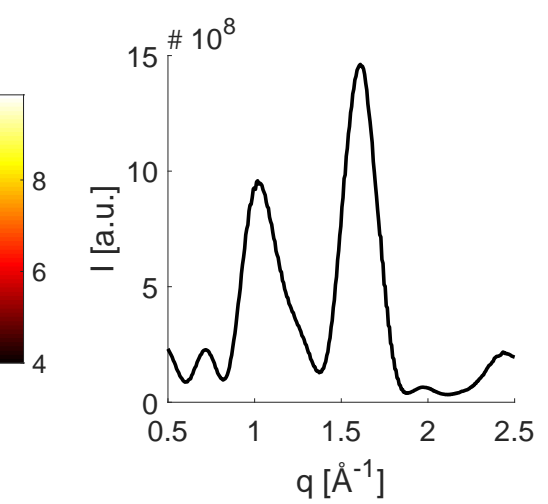

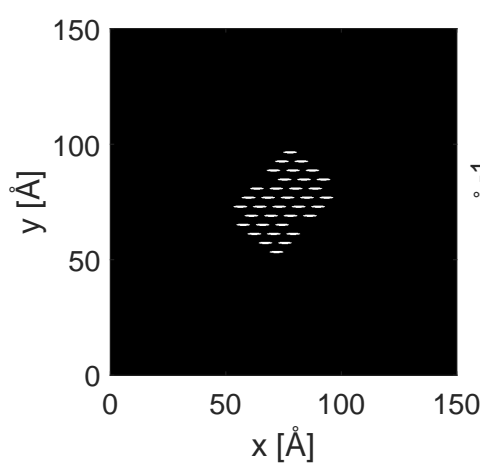
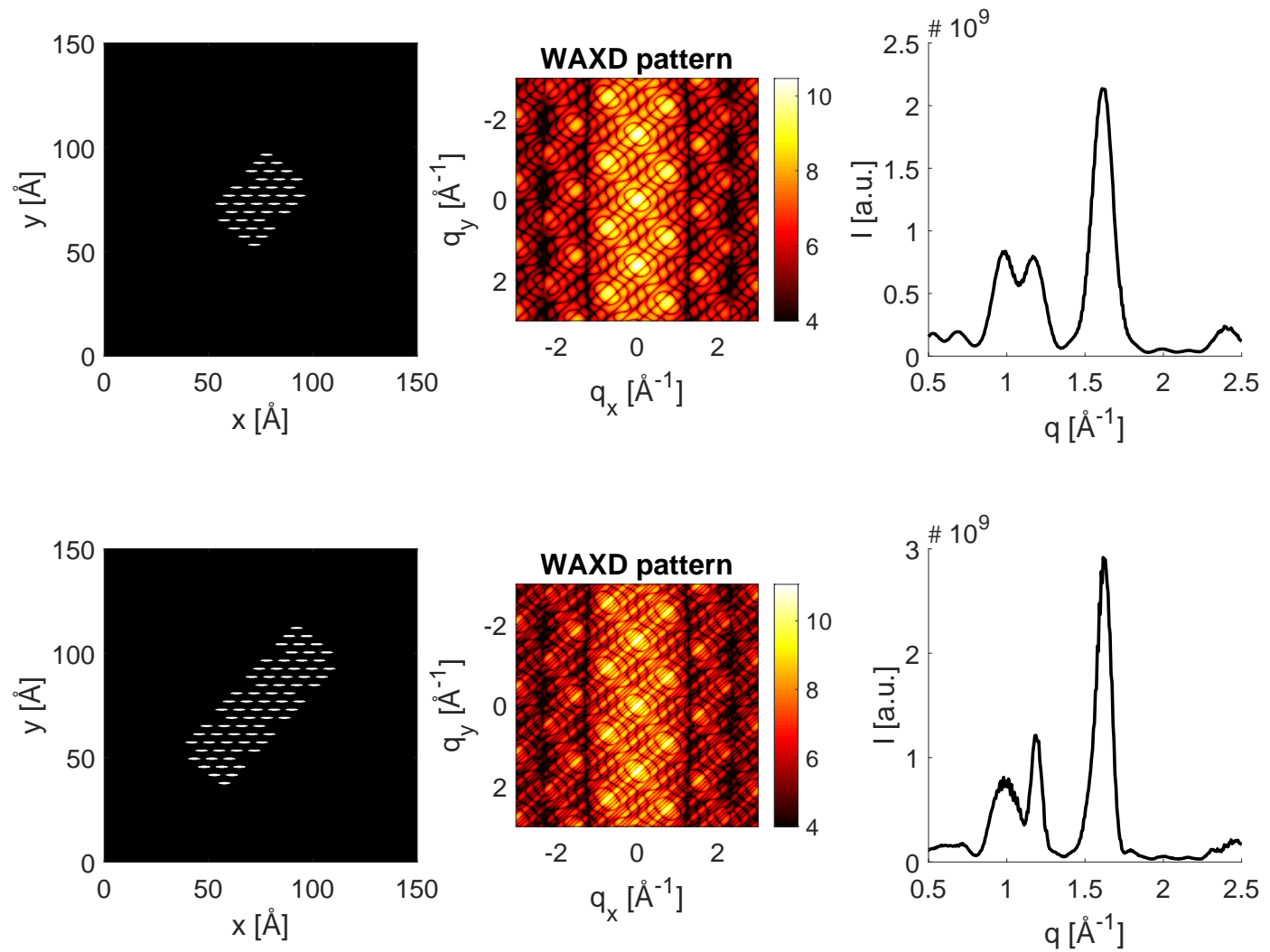
Model: H2-

Aggregation direction: (1-10)

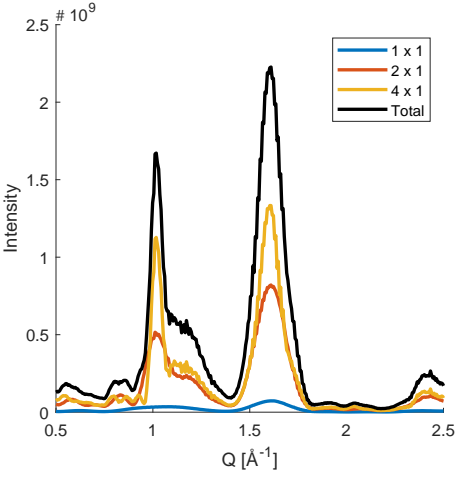

Sample \#1

Vol\% 1x1: 4.7

Vol\% 2x1: 42.3

Vol\% 4x1: 53.0
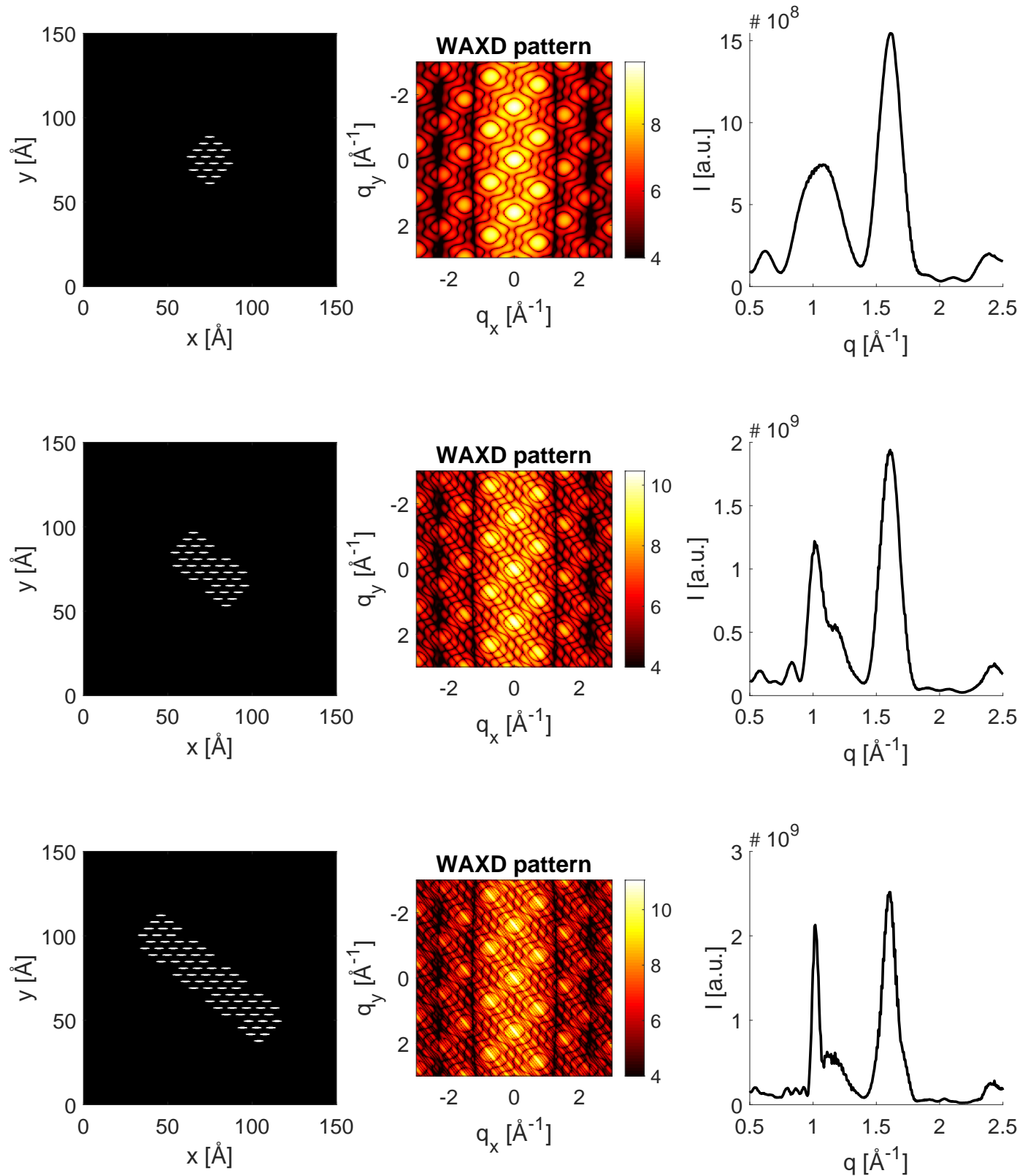
Model: H2-

Aggregation direction: (110)

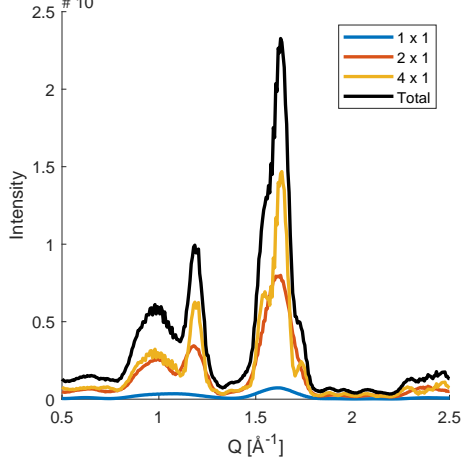

Sample \#1

Vol\% 1x1: 4.7

Vol\% 2x1: 42.3

Vol\% 4x1: 53.0
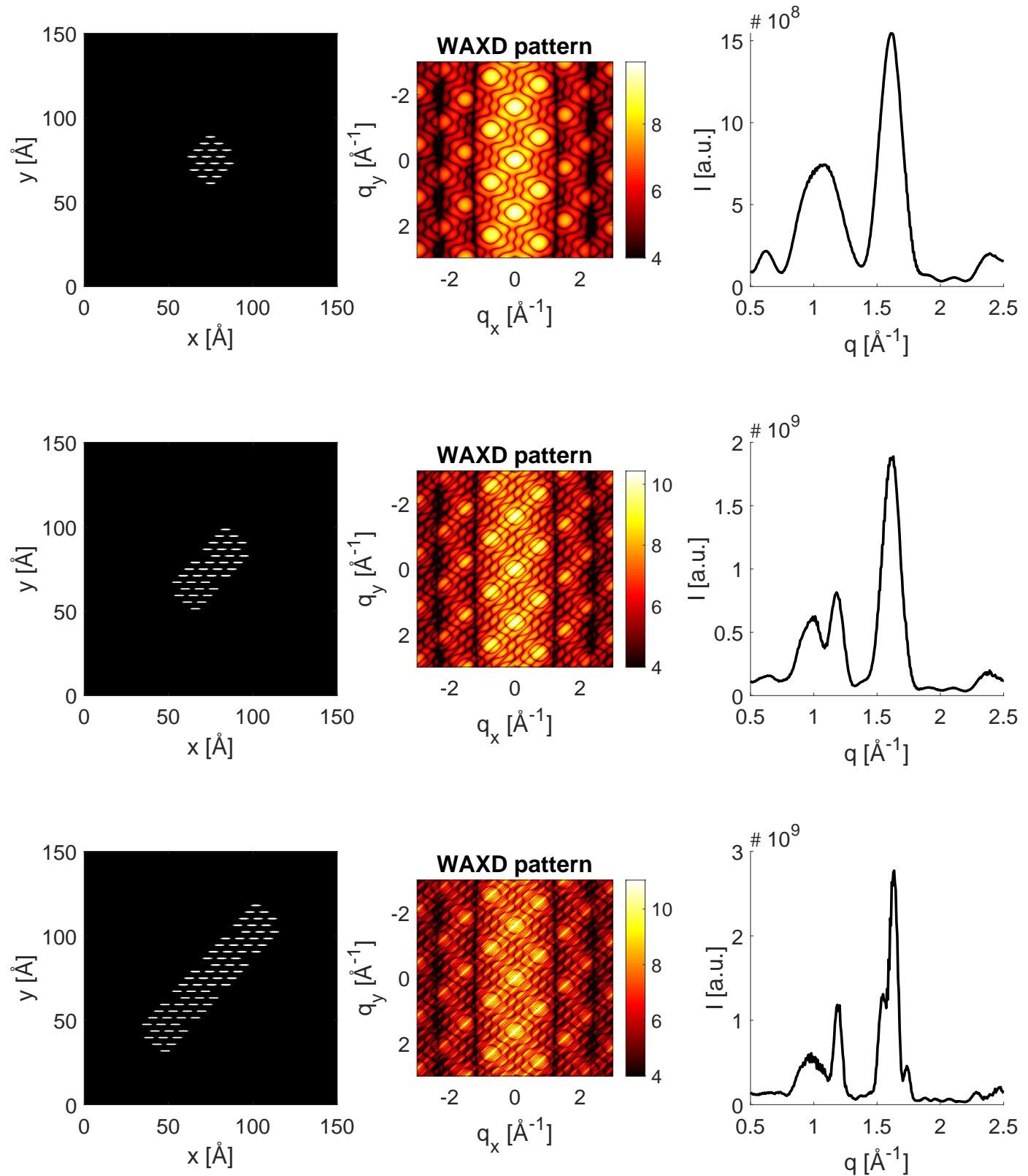
Model: R1+

Aggregation direction: (1-10)

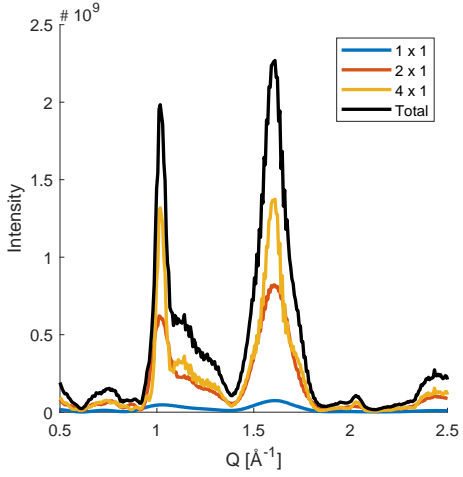

Sample \#1

Vol\% 1x1: 4.7

Vol\% 2x1: 42.3

Vol\% 4x1: 53.0
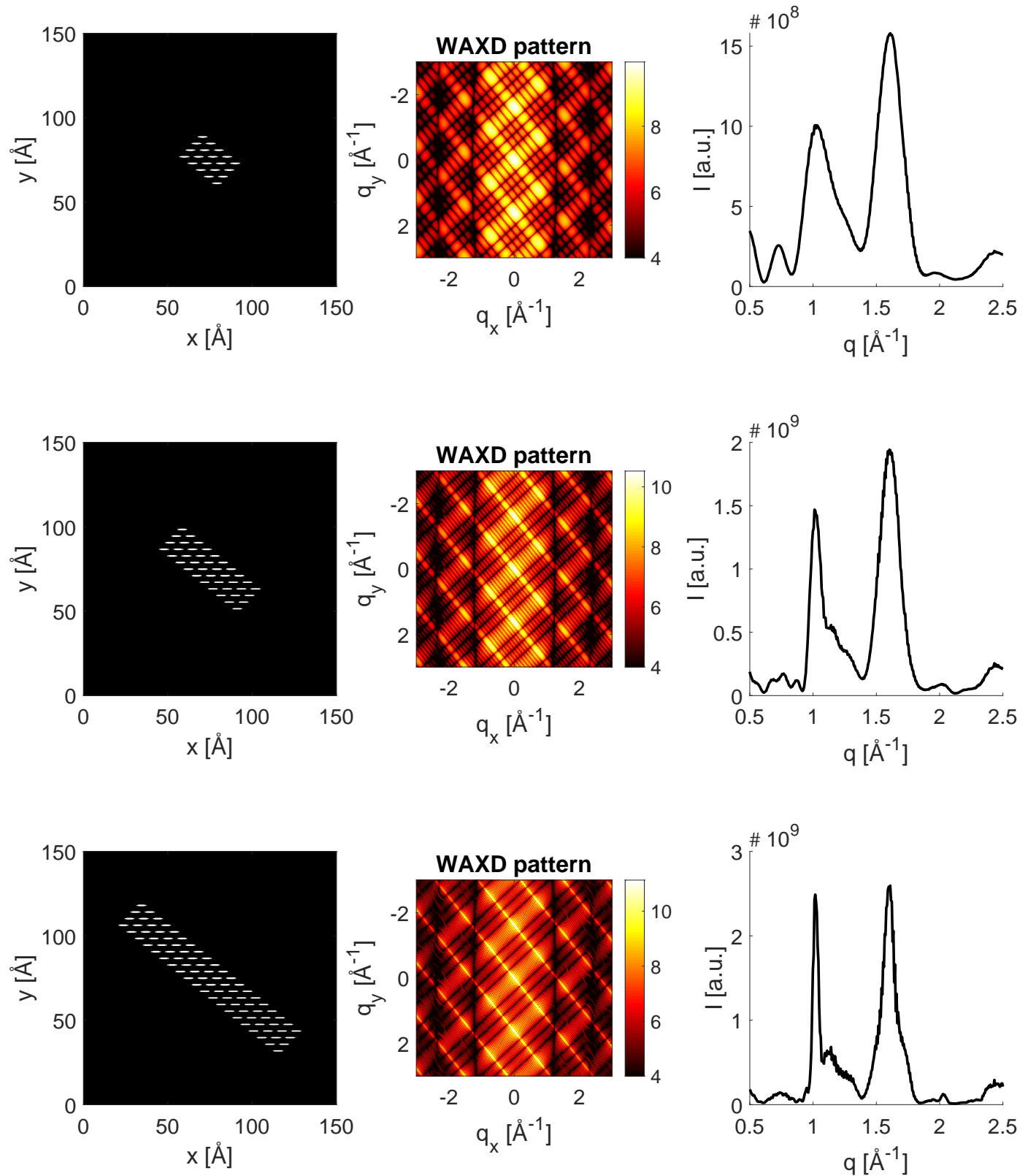
Model: R1+

Aggregation direction: (110)

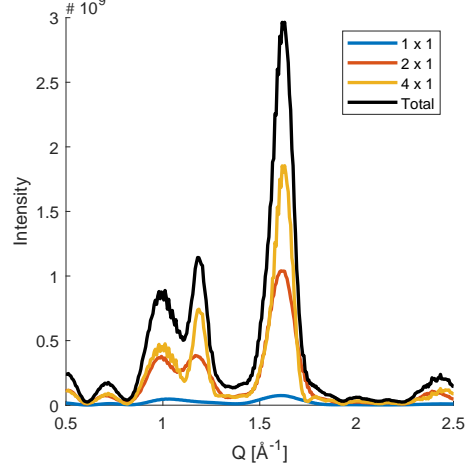

Sample \#1

Vol\% 1x1: 4.7

Vol\% $2 \times 1: 42.3$

Vol\% $4 \times 1: 53.0$
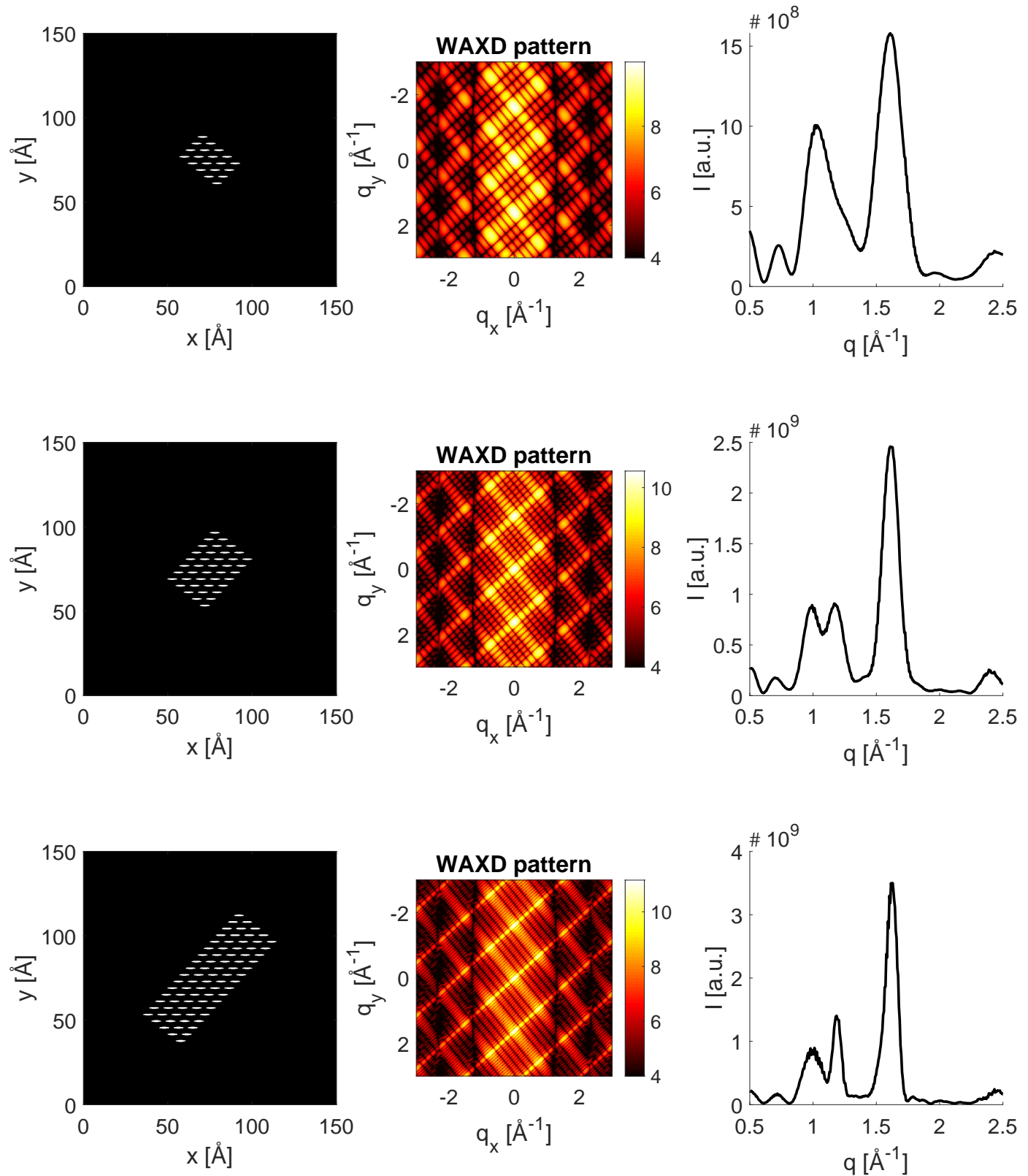
Model: R1-

Aggregation direction: (1-10)

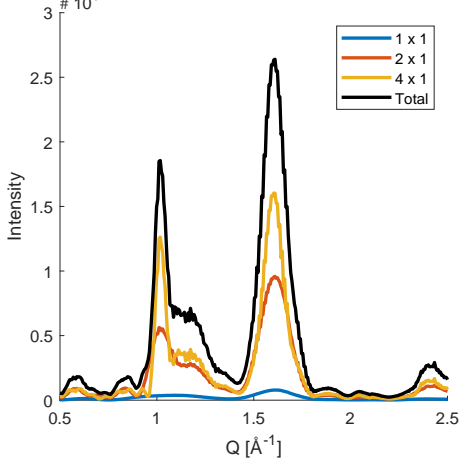

Sample \#1

Vol\% 1x1: 4.7

Vol\% $2 \times 1: 42.3$

Vol\% 4x1: 53.0
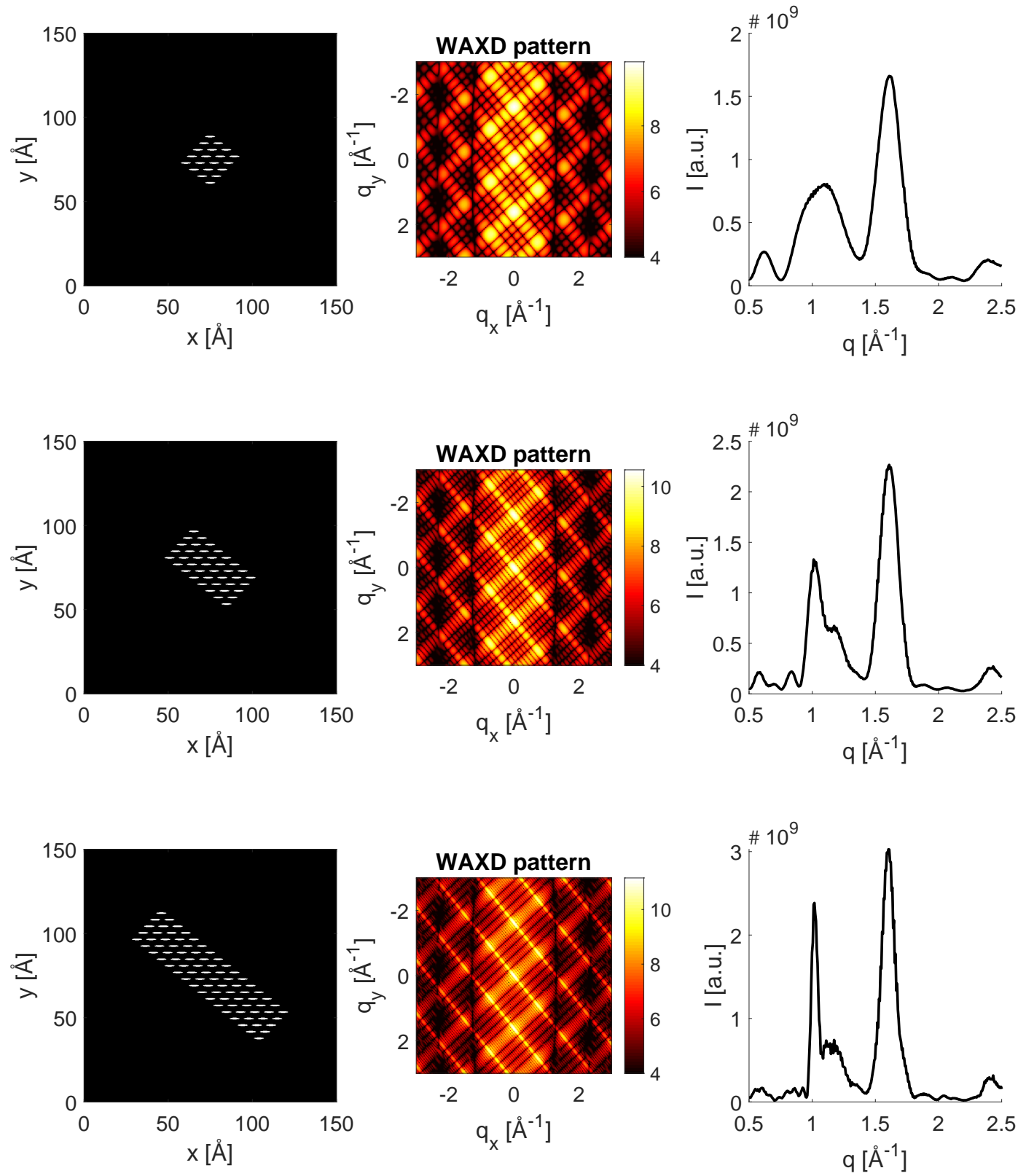
Model: R1-

Aggregation direction: (110)

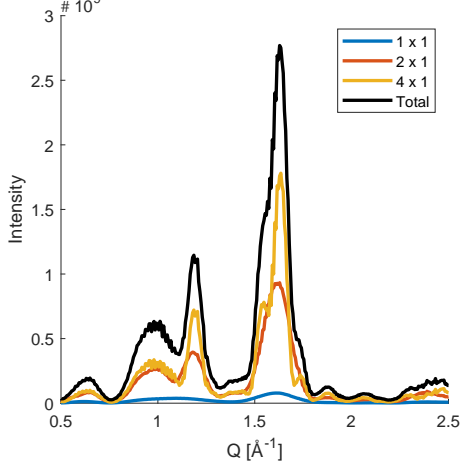

Sample \#1

Vol\% 1x1: 4.7

Vol\% 2x1: 42.3

Vol\% $4 \times 1: 53.0$
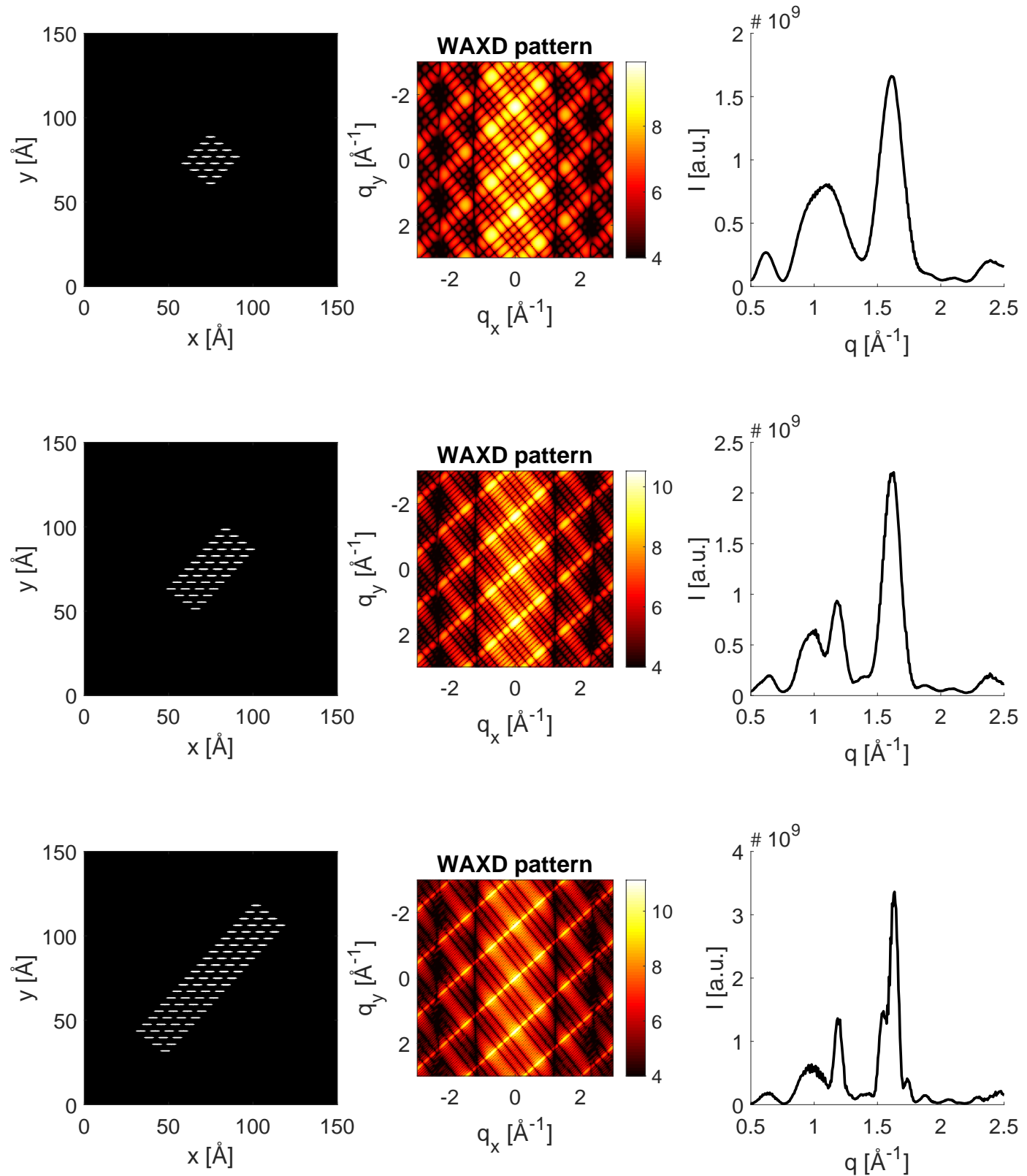
Model: R2+

Aggregation direction: (1-10)

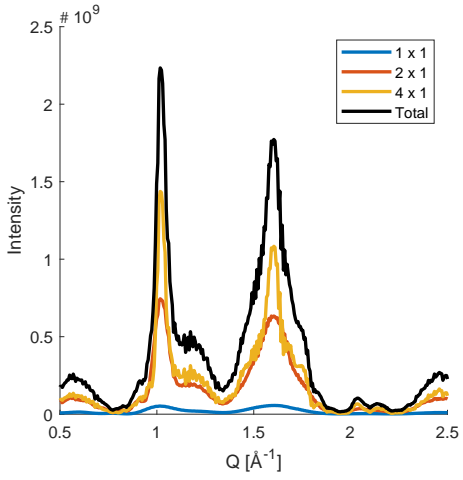

Sample \#1

Vol\% 1x1: 4.7

Vol\% 2x1: 42.3

Vol\% 4x1: 53.0
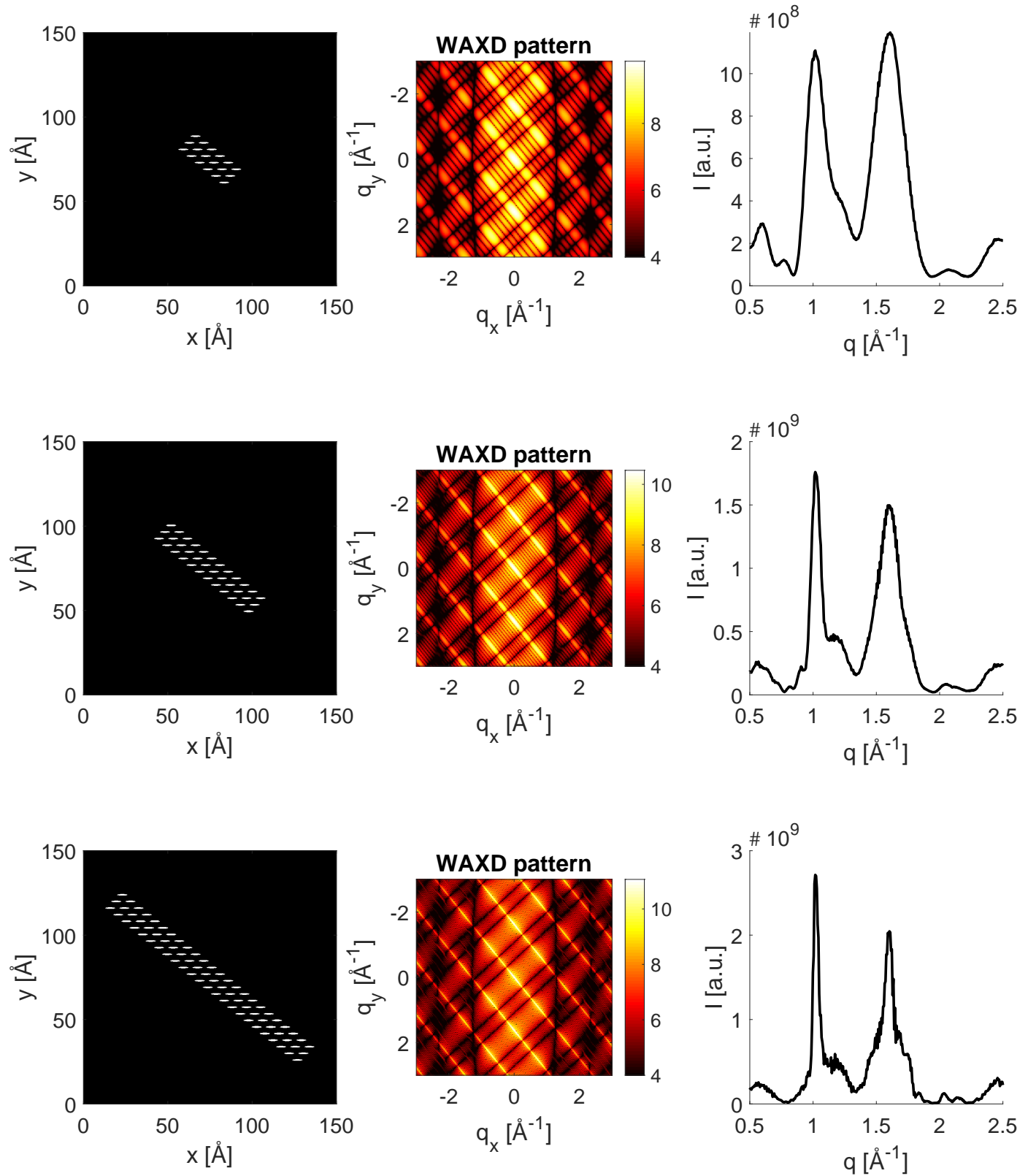
Model: R2+

Aggregation direction: (110)

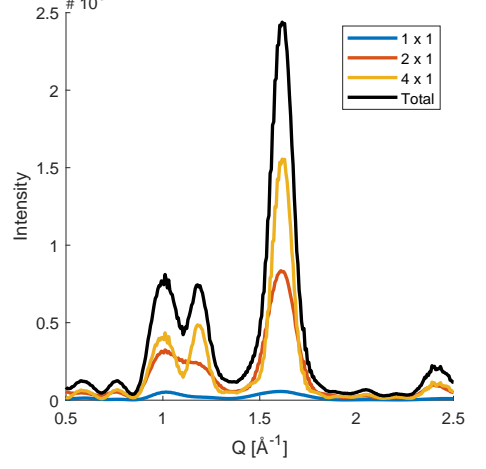

Sample \#1

Vol\% 1x1: 4.7

Vol\% $2 \times 1: 42.3$

Vol\% $4 \times 1: 53.0$
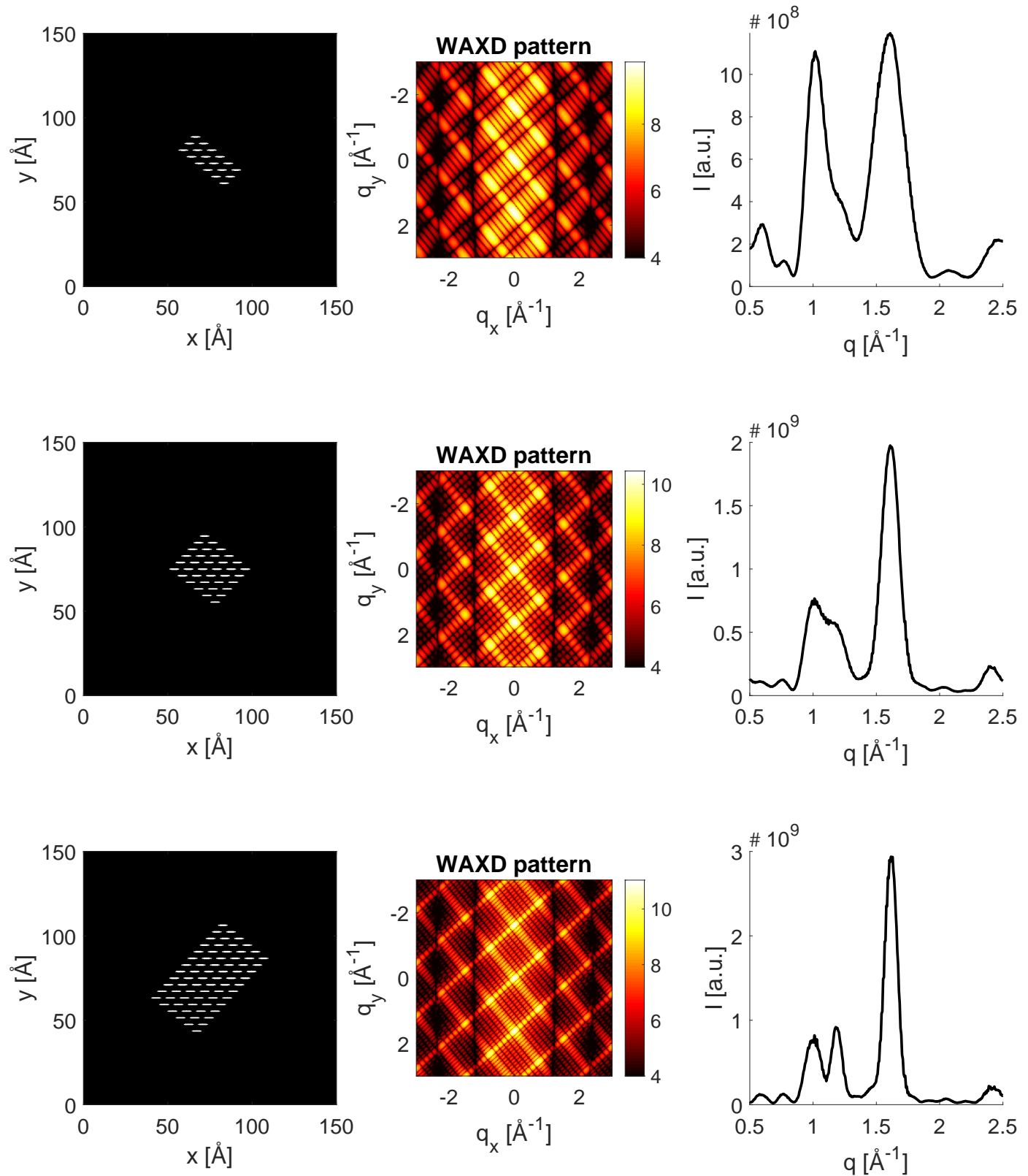
Model: R2-

Aggregation direction: (1-10)

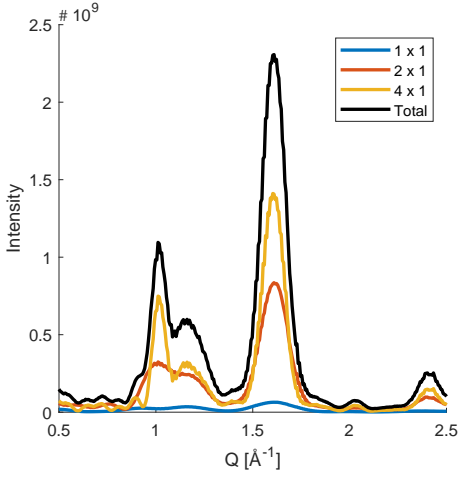

Sample \#1

Vol\% 1x1: 4.7

Vol\% $2 \times 1: 42.3$

Vol\% 4x1: 53.0
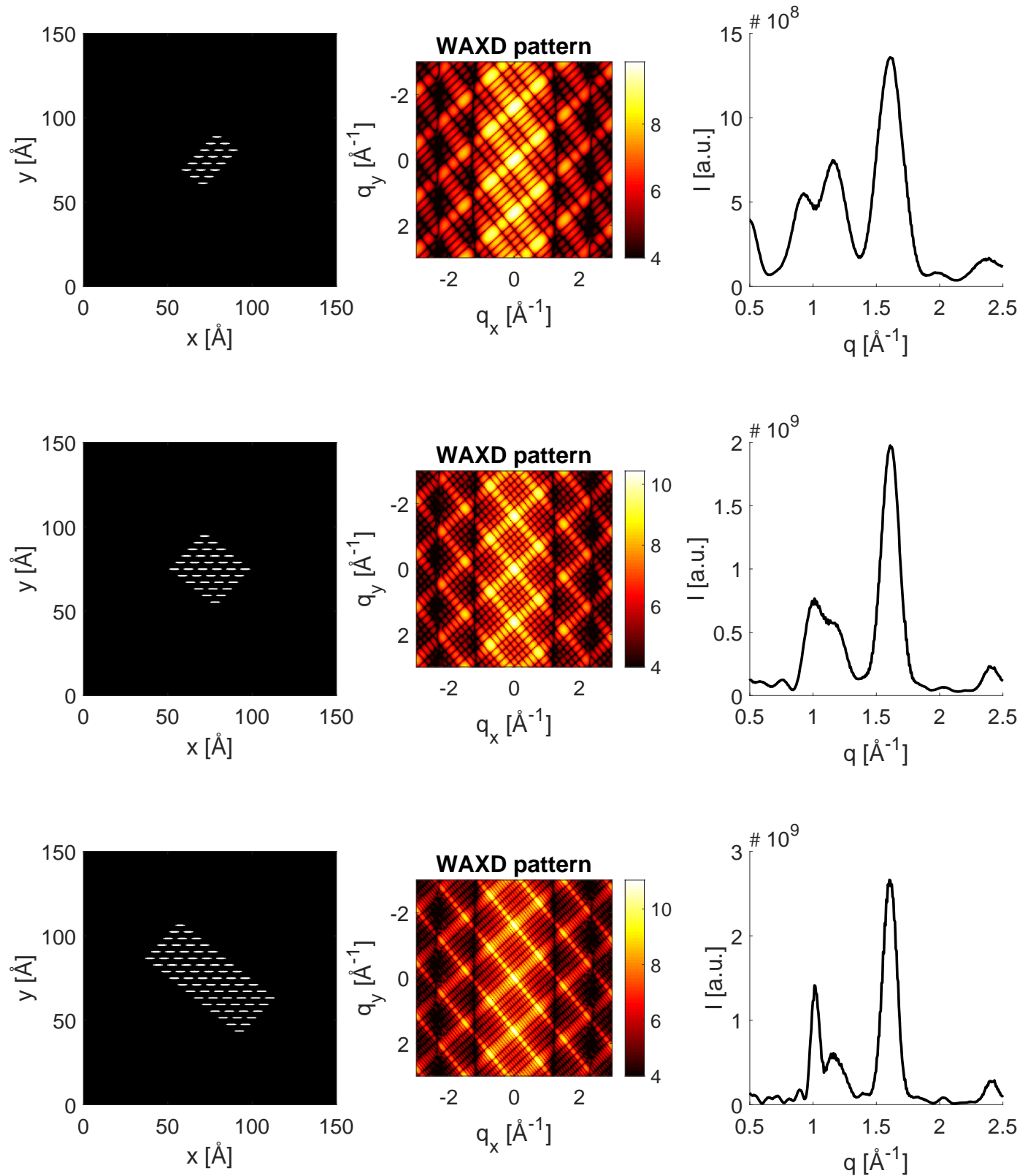
Model: R2-

Aggregation direction: (110)

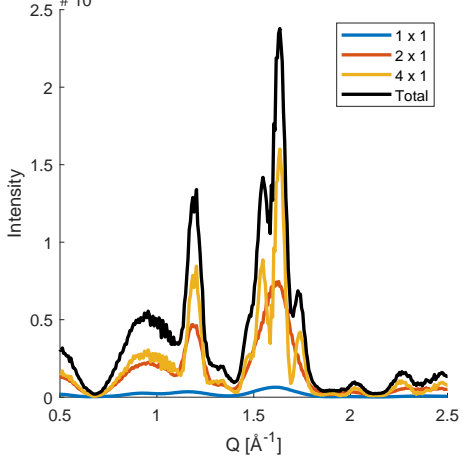

Sample \#1

Vol\% 1x1: 4.7

Vol\% 2x1: 42.3

Vol\% 4x1: 53.0
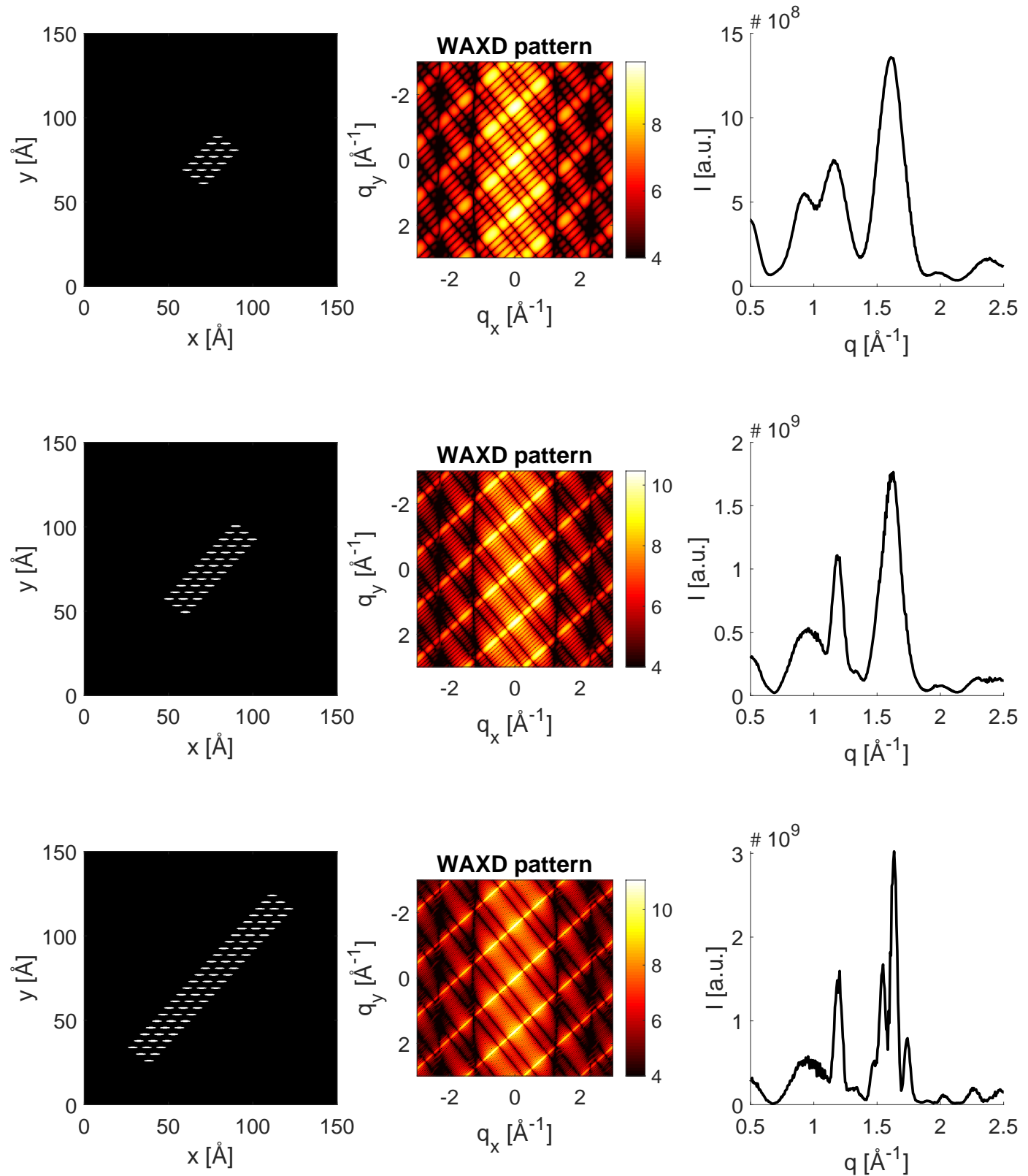
Model: Rh1+

Aggregation direction: (110)
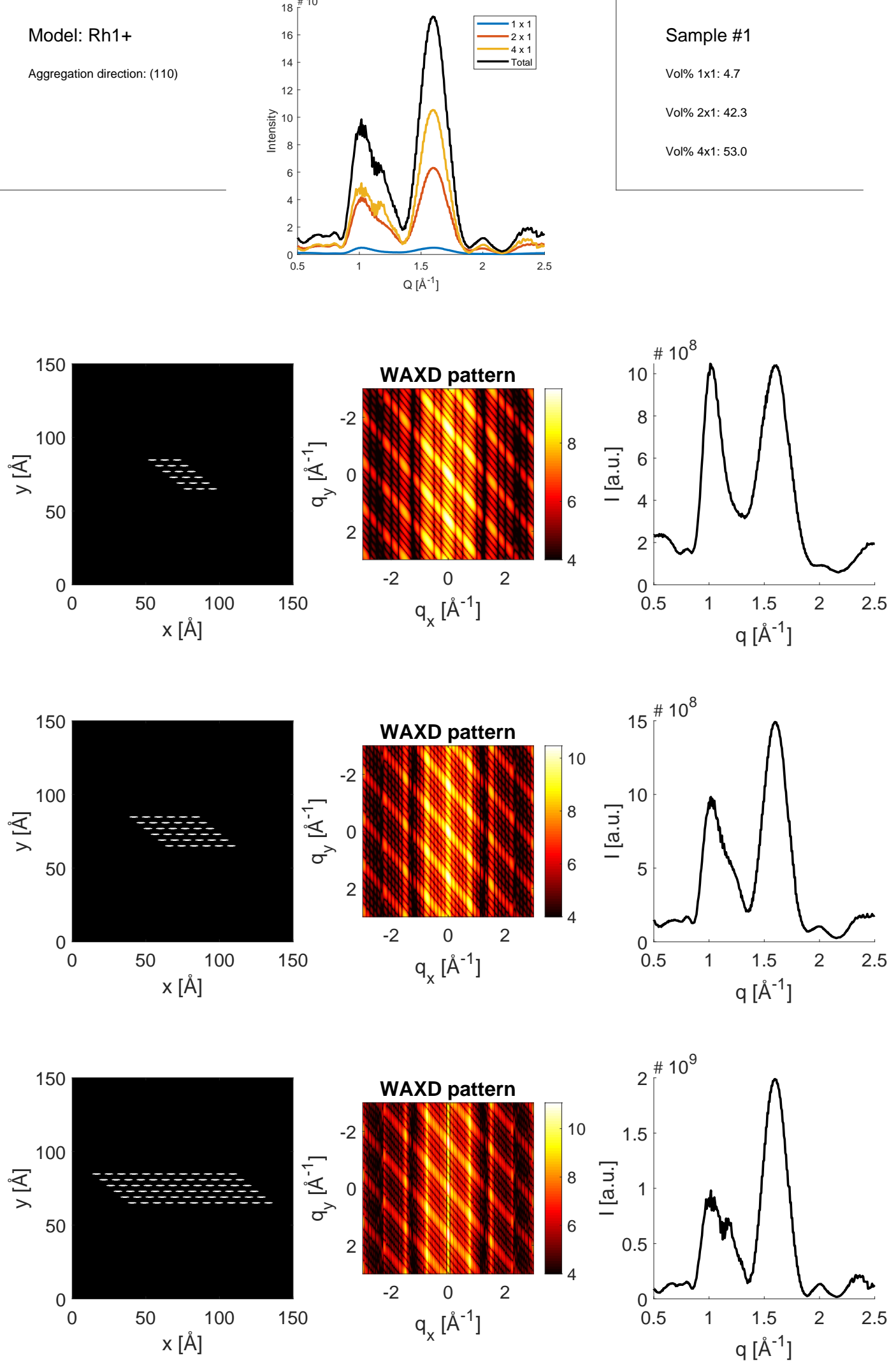

Sample \#1

Vol\% $1 \times 1: 4.7$

Vol\% $2 \times 1: 42.3$

Vol\% 4x1: 53.0 
Model: Rh1+

Aggregation direction: (200)

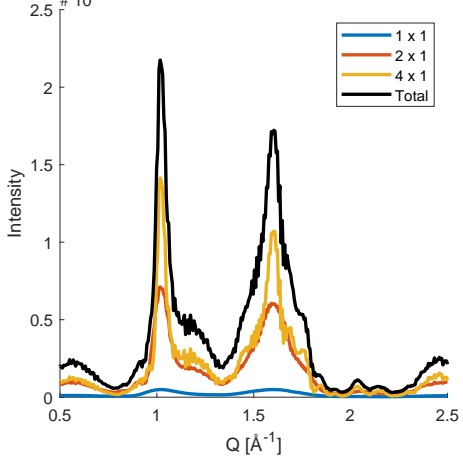

Sample \#1

Vol\% 1x1: 4.7

Vol\% $2 \times 1: 42.3$

Vol\% 4x1: 53.0
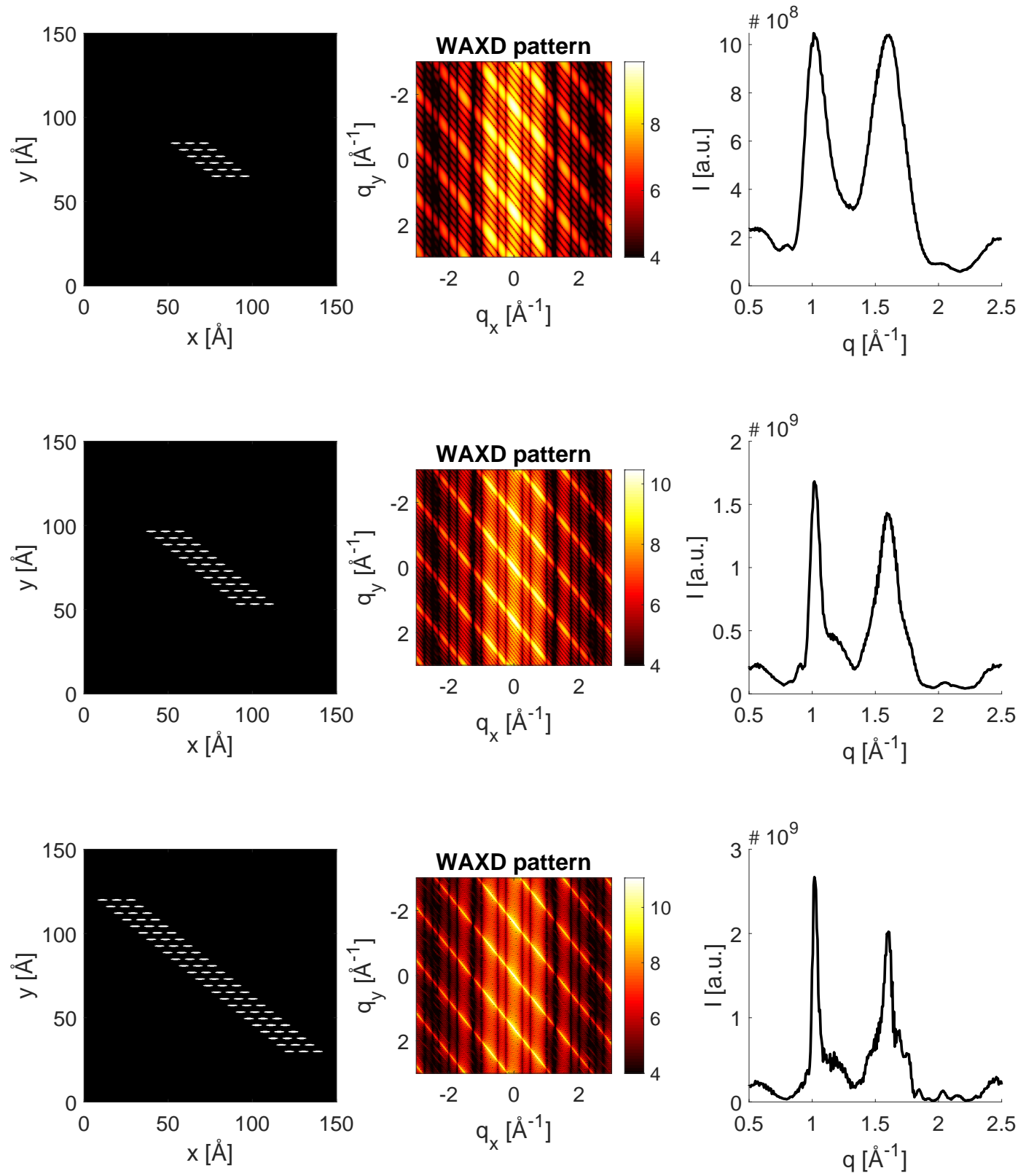
Model: Rh1-

Aggregation direction: (1-10)

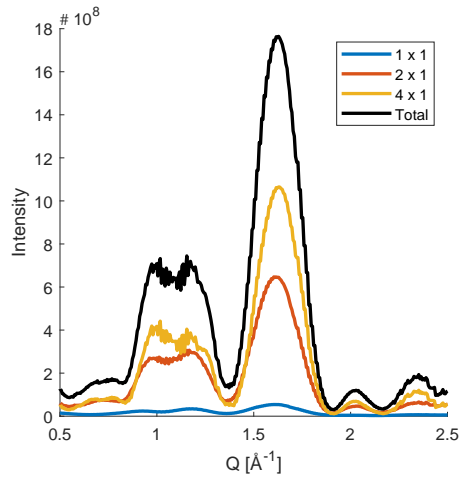

Sample \#1

Vol\% 1x1: 4.7

Vol\% 2x1: 42.3

Vol\% 4x1: 53.0
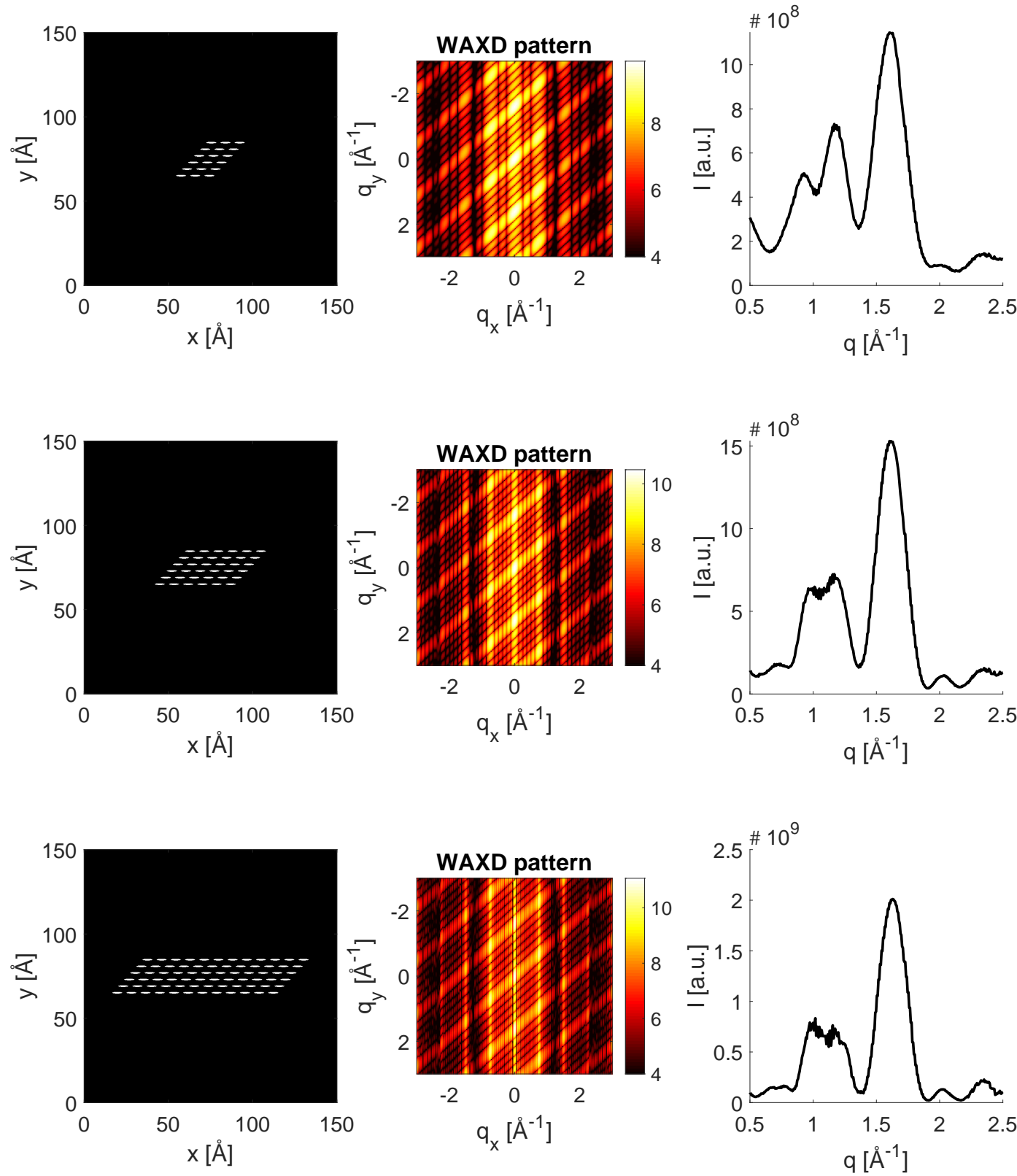
Model: Rh1-

Aggregation direction: (200)

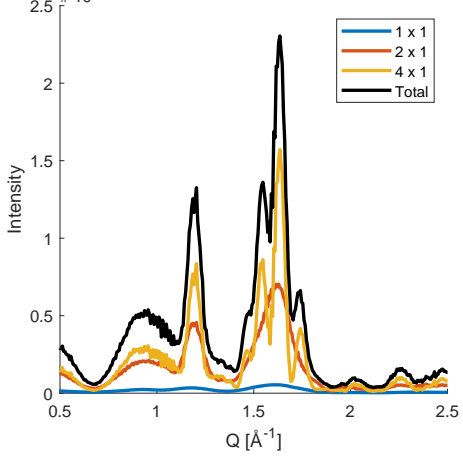

Sample \#1

Vol\% 1x1: 4.7

Vol\% 2x1: 42.3

Vol\% 4x1: 53.0
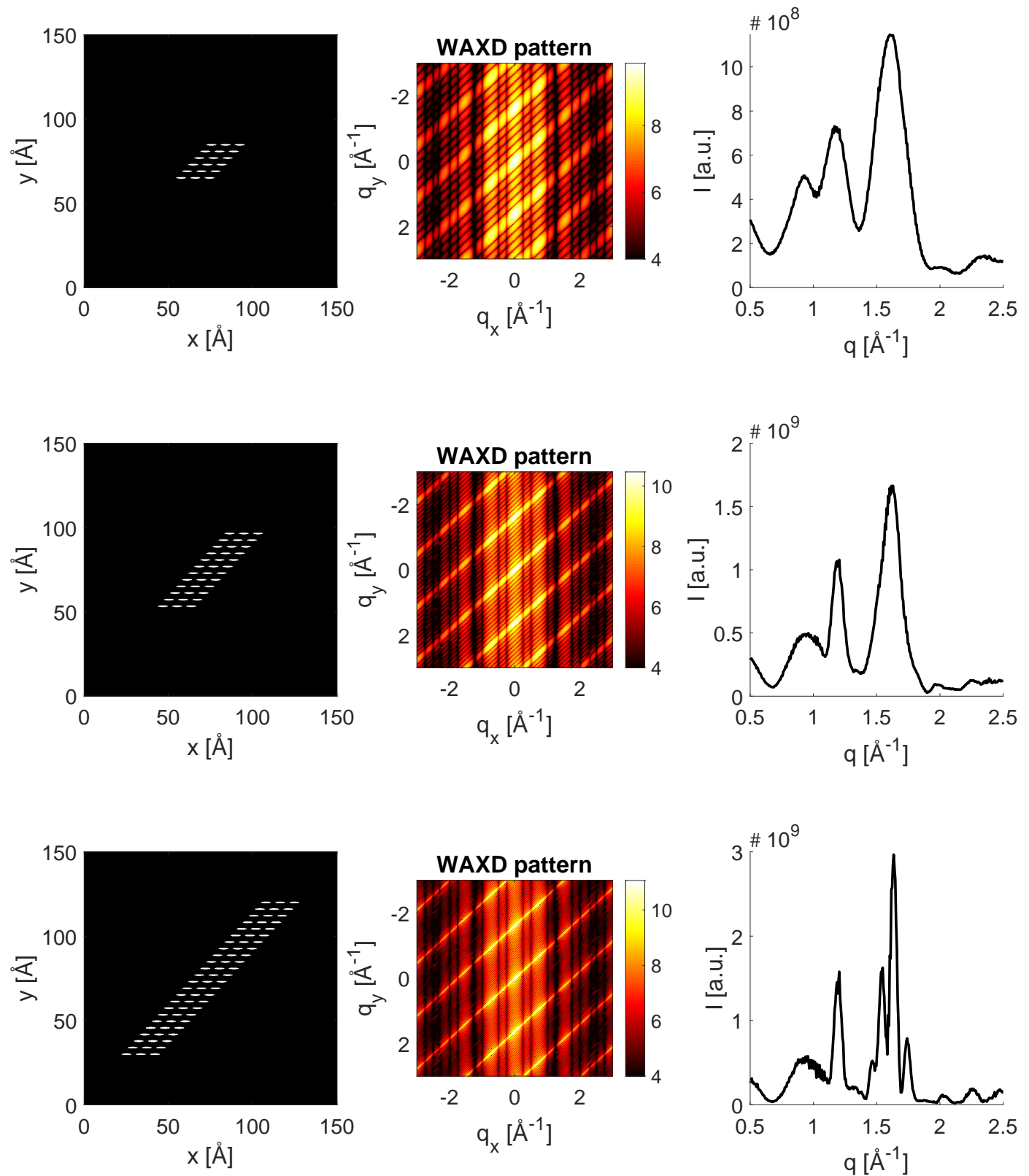
Model: Rh2

Aggregation direction: (010)

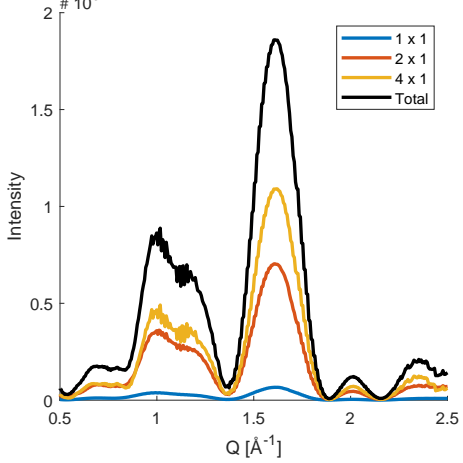

Sample \#1

Vol\% 1x1: 4.7

Vol\% $2 \times 1: 42.3$

Vol\% $4 \times 1: 53.0$
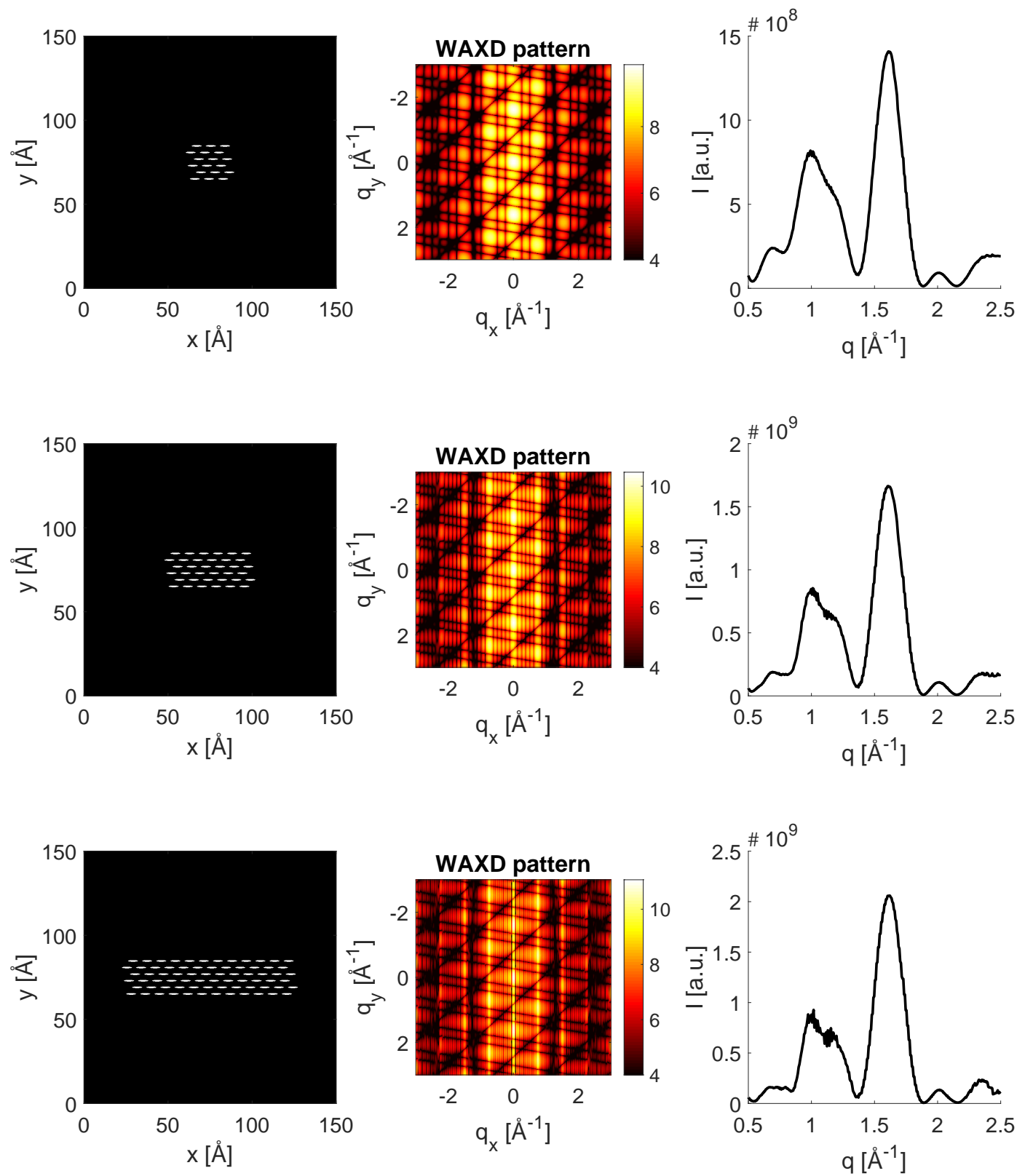
Model: Rh2

Aggregation direction: (200)

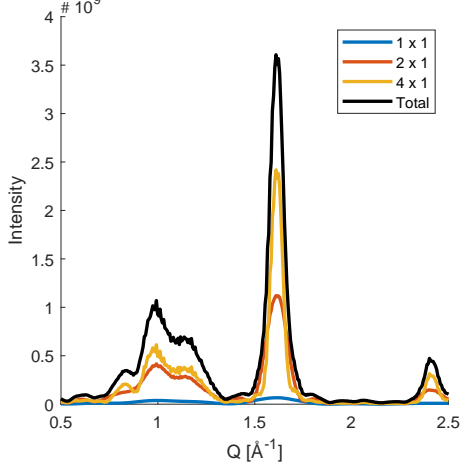

Sample \#1

Vol\% 1x1: 4.7

Vol\% 2x1: 42.3

Vol\% $4 \times 1: 53.0$
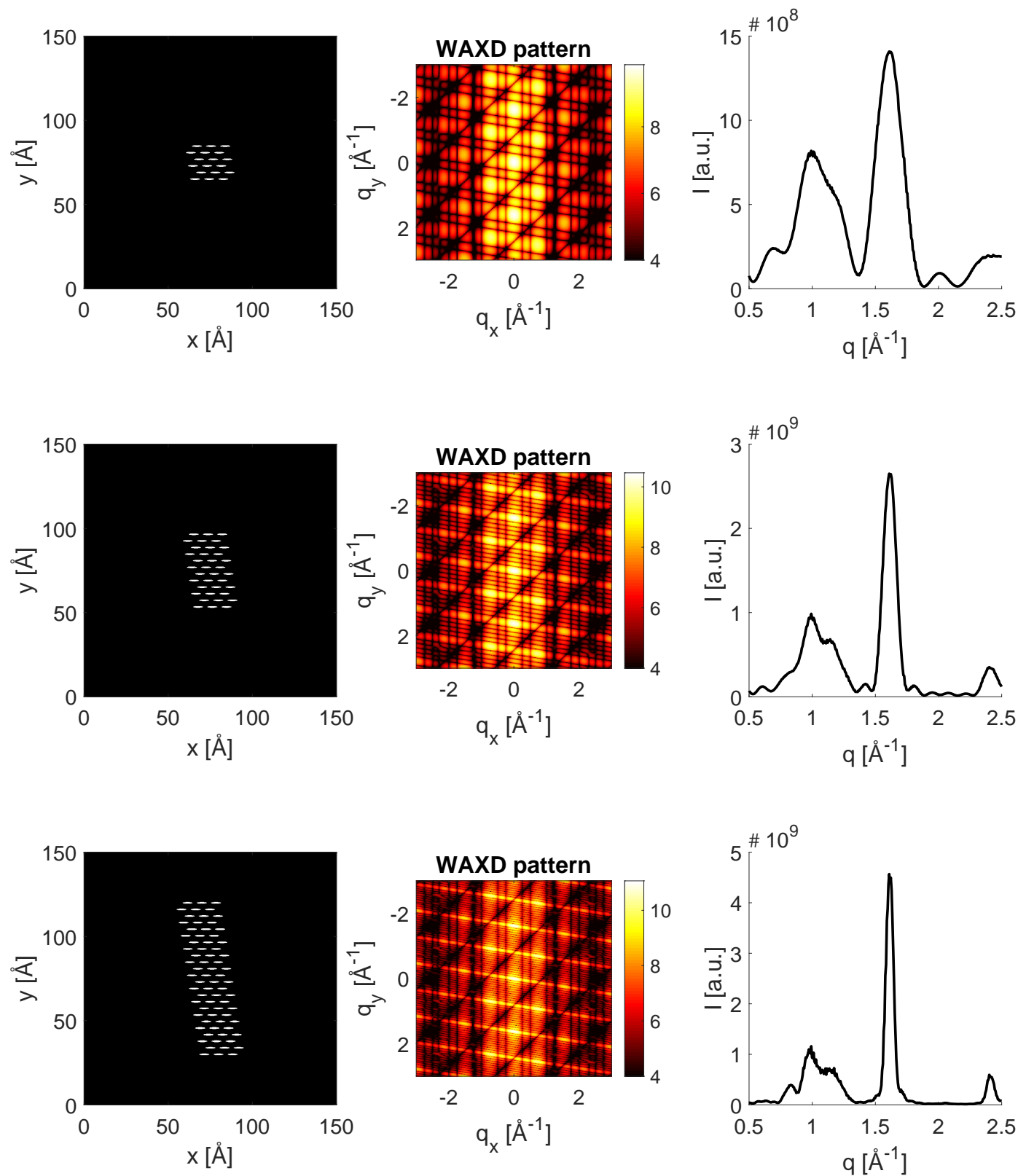

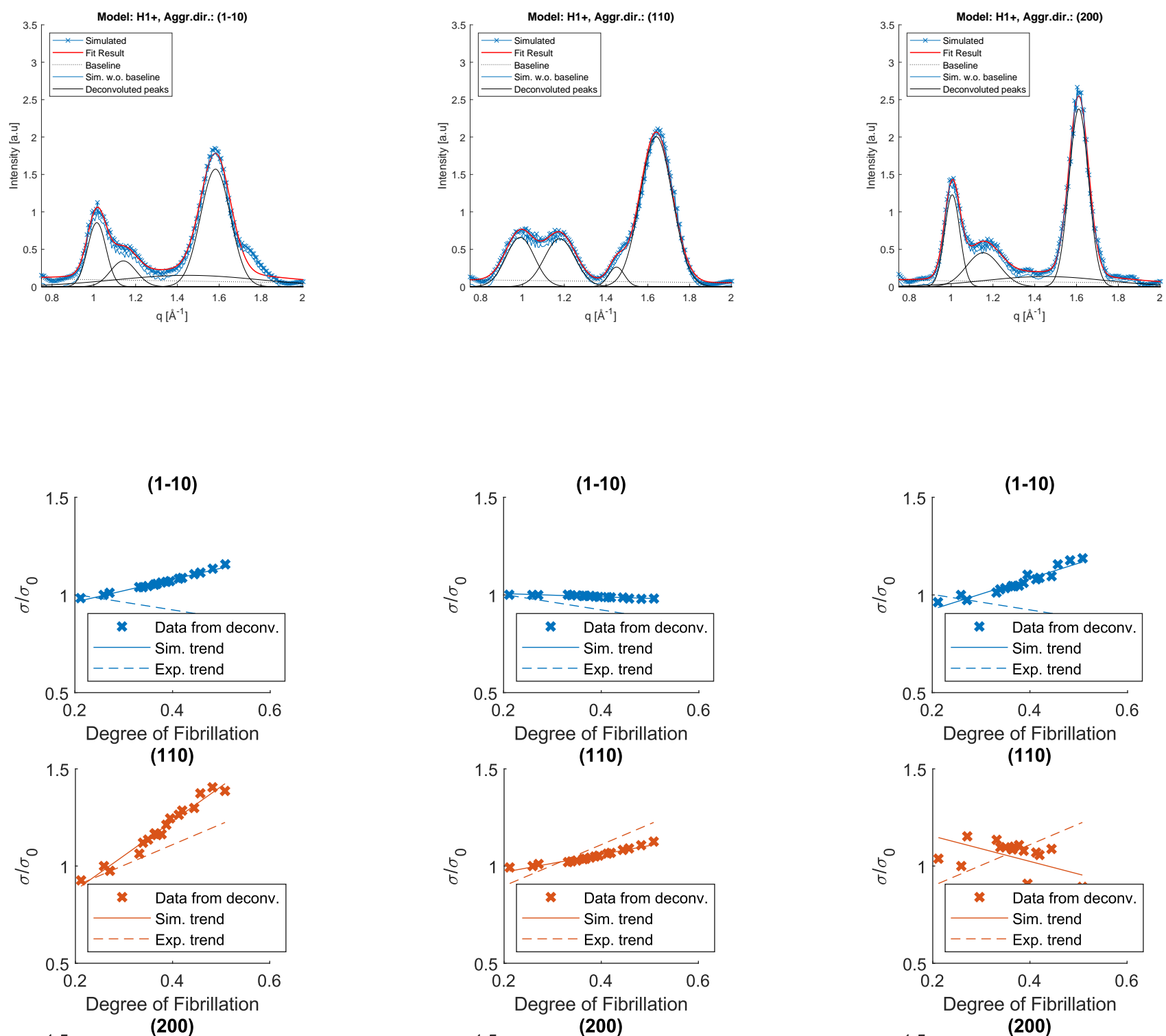

(110)
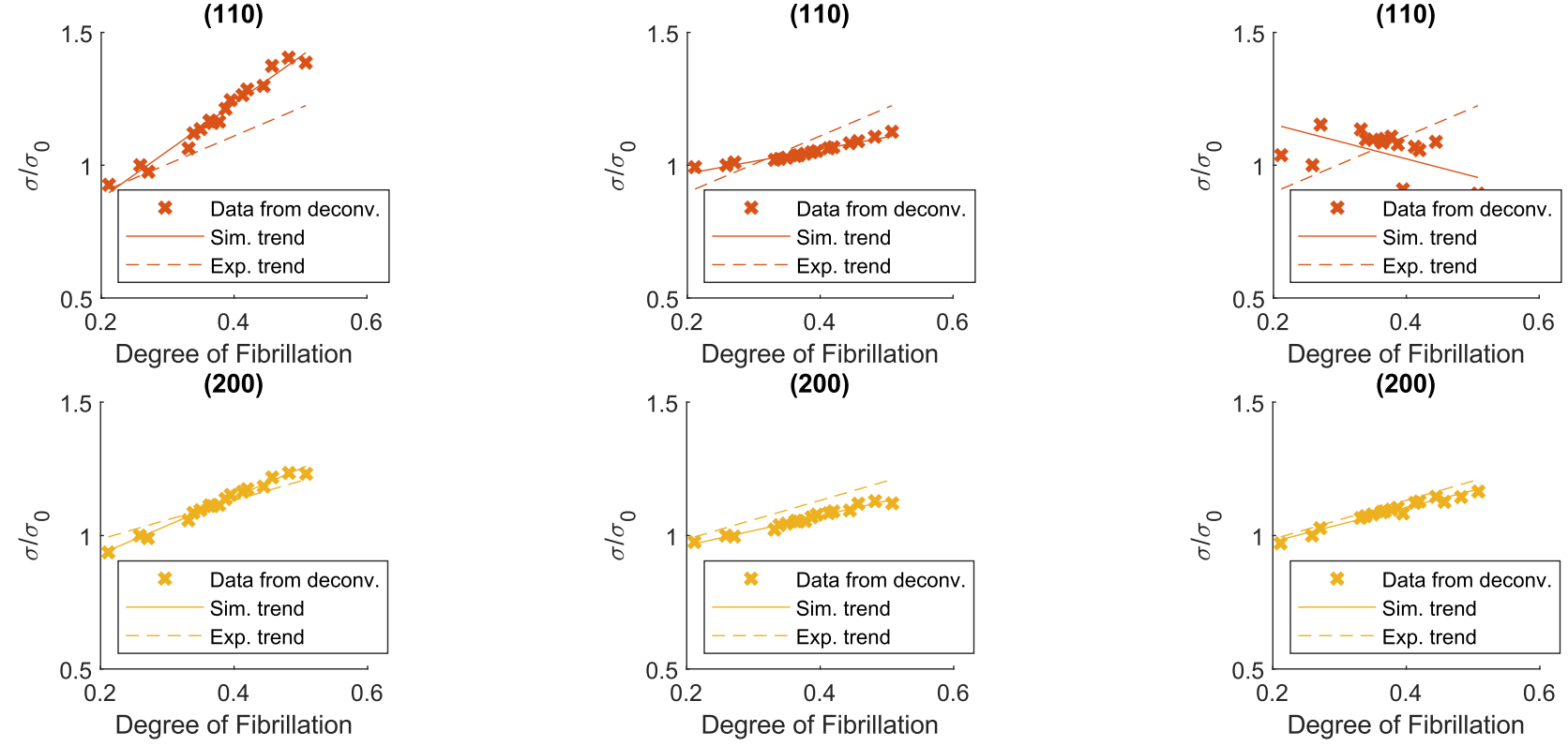

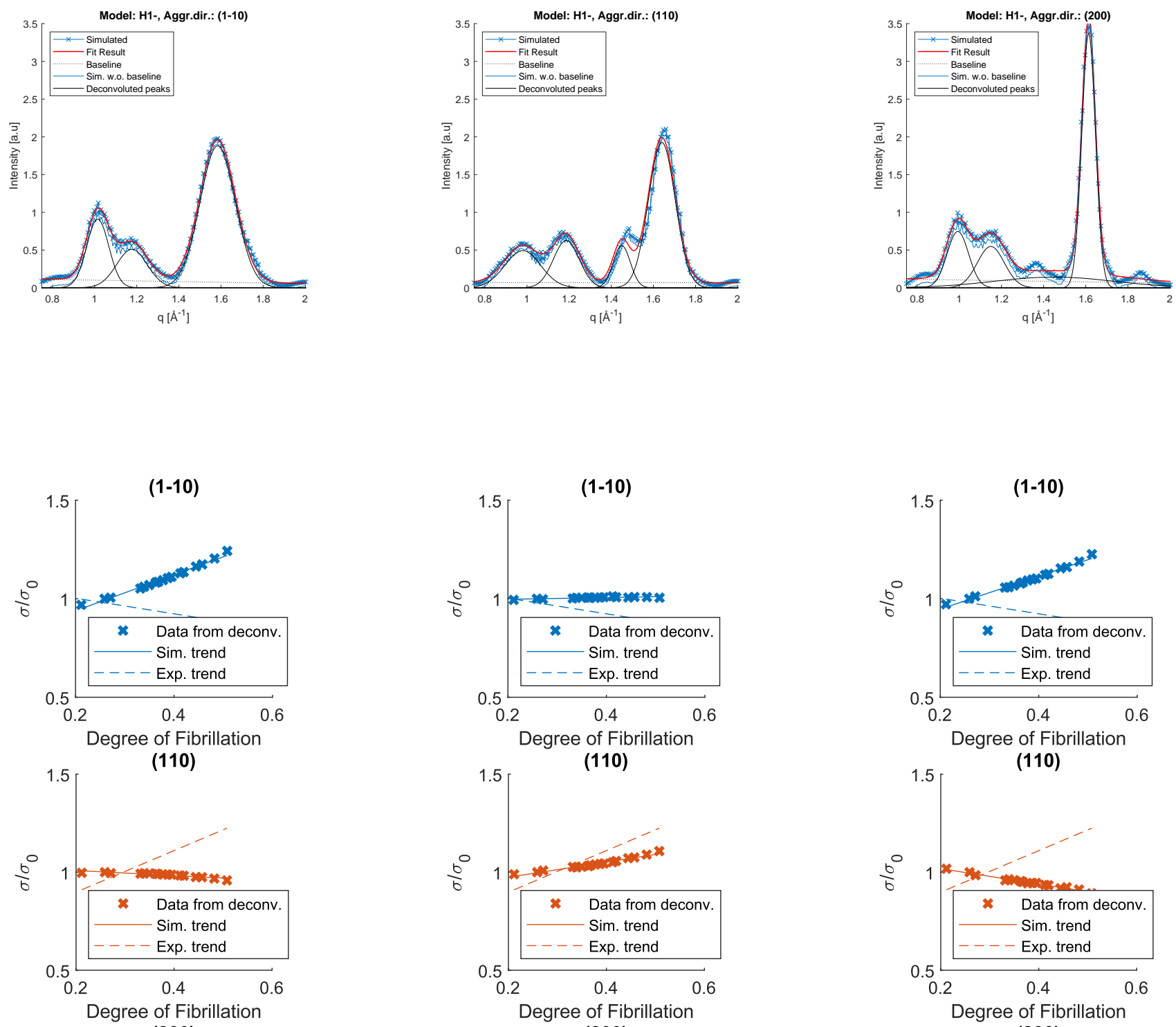

(110)
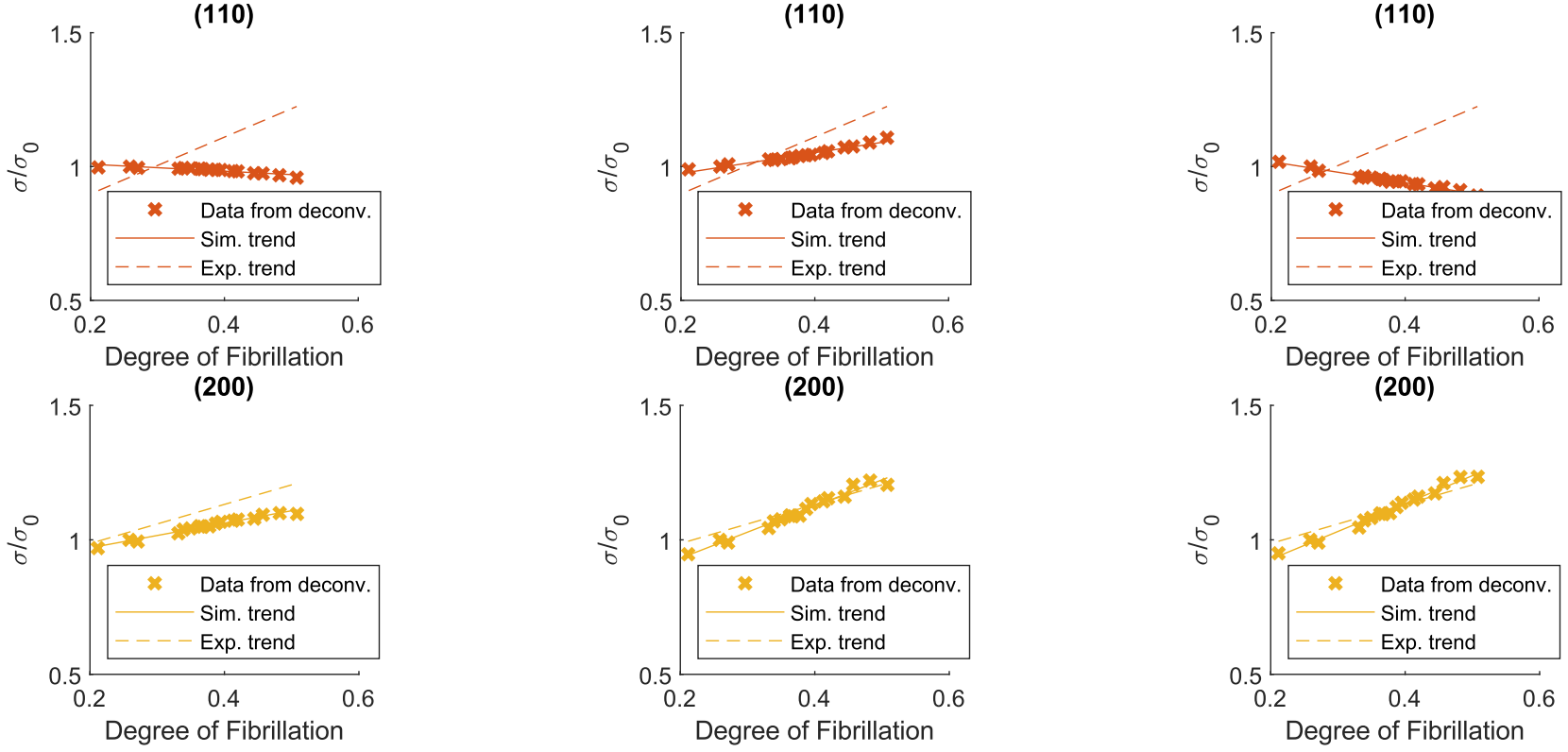

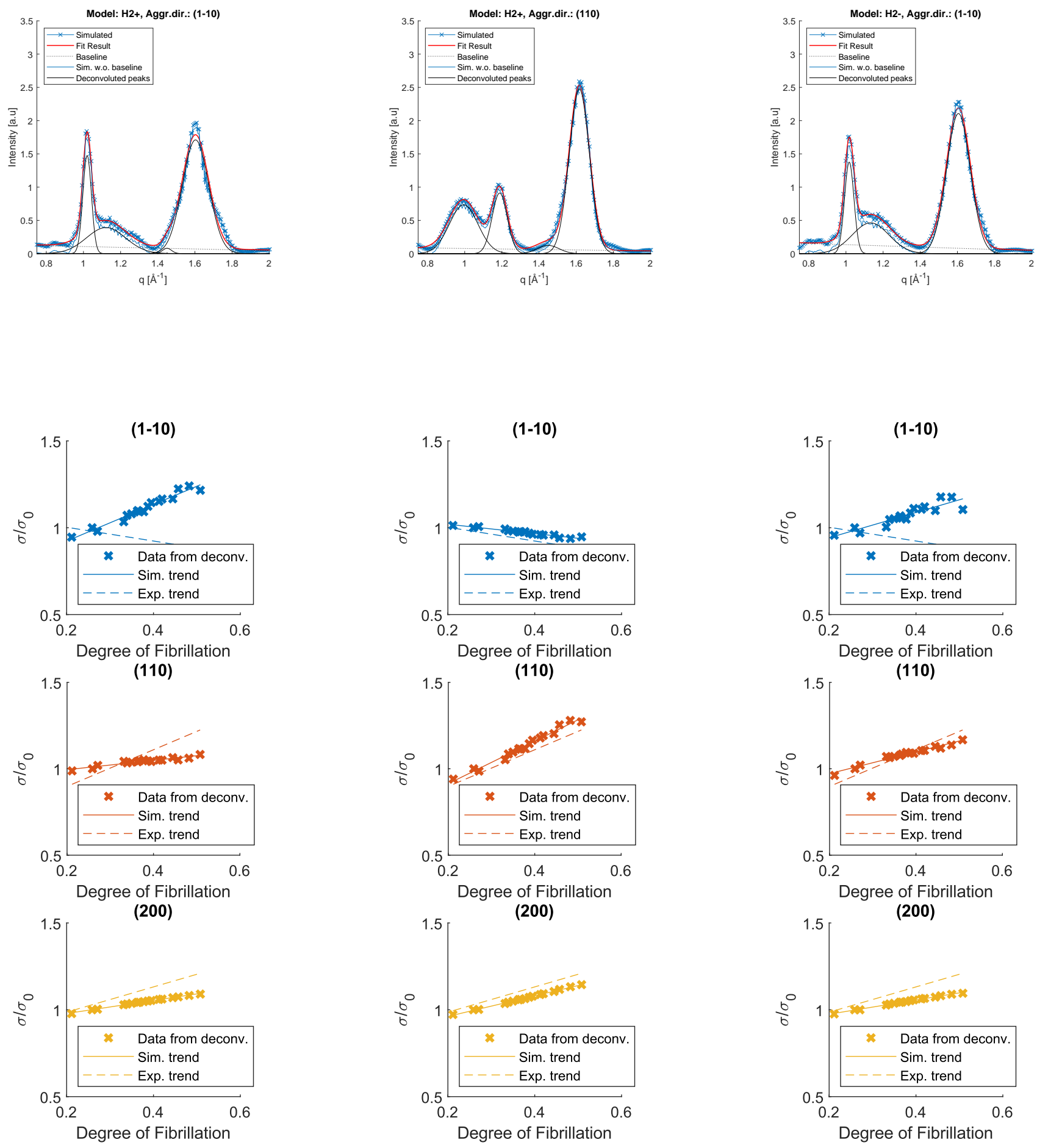

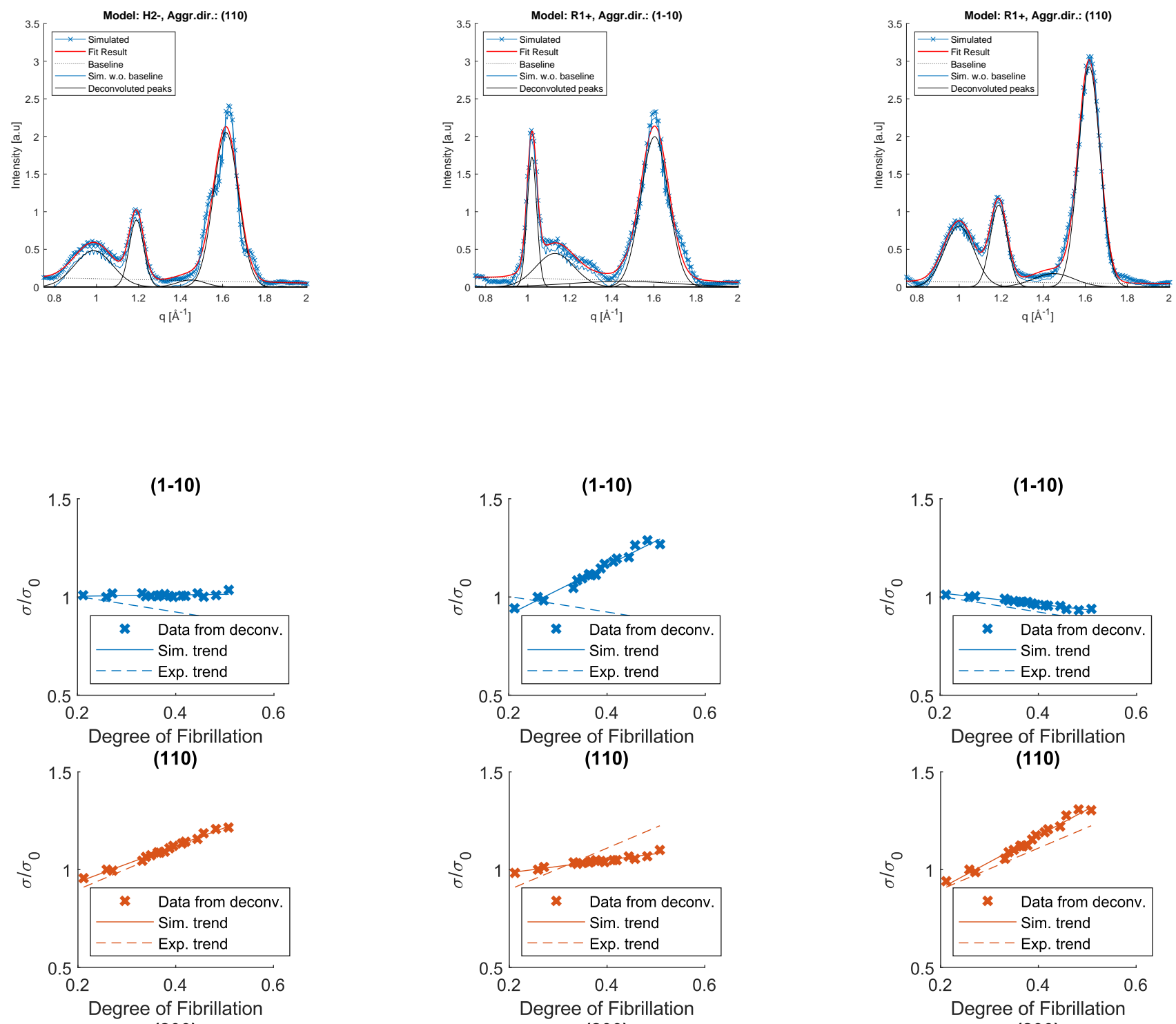

(110)

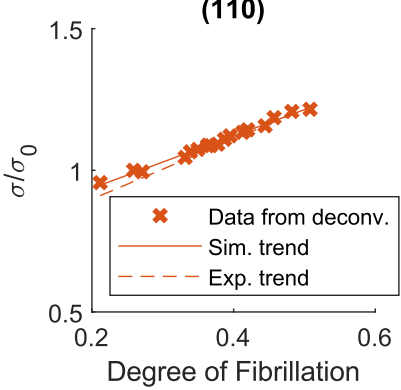

(200)
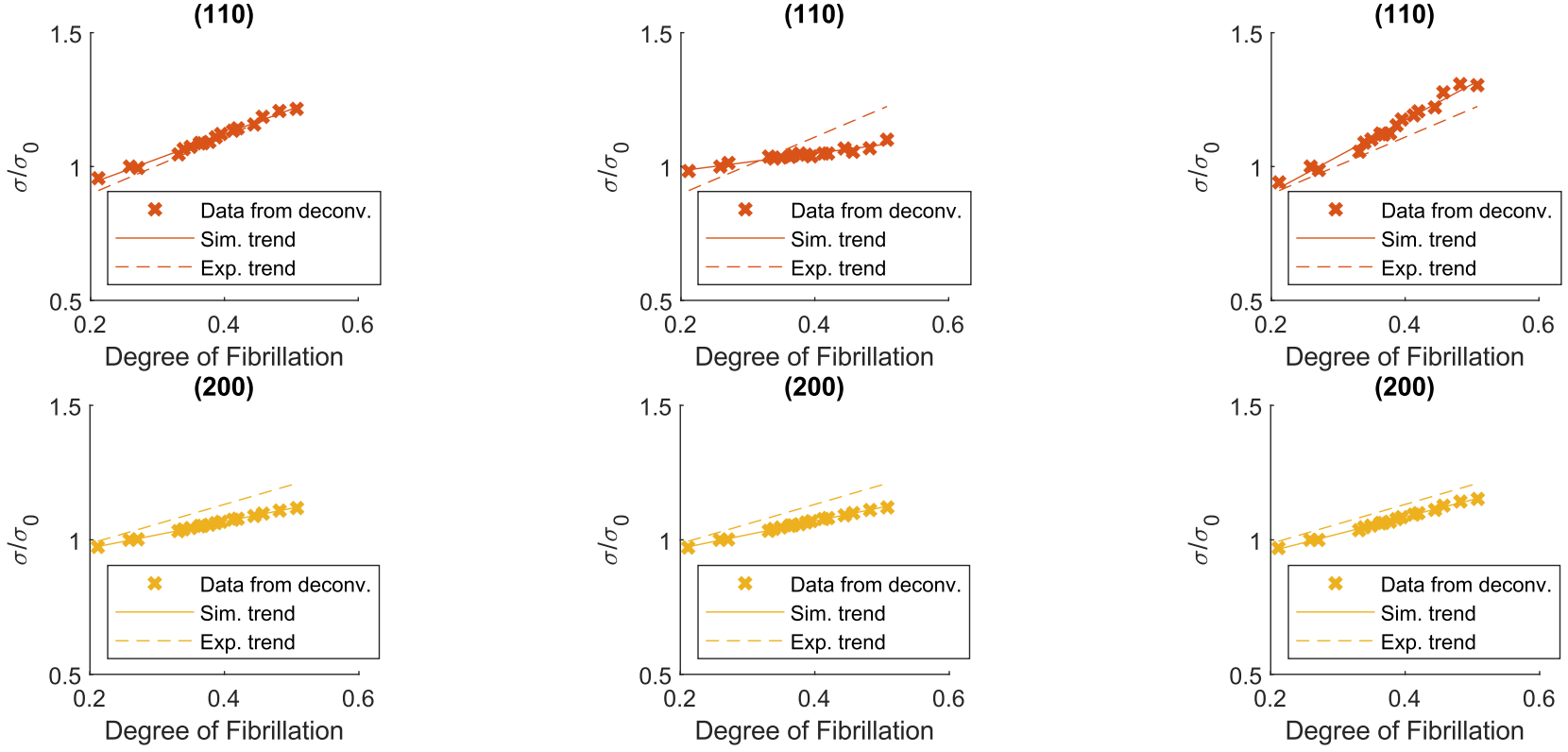

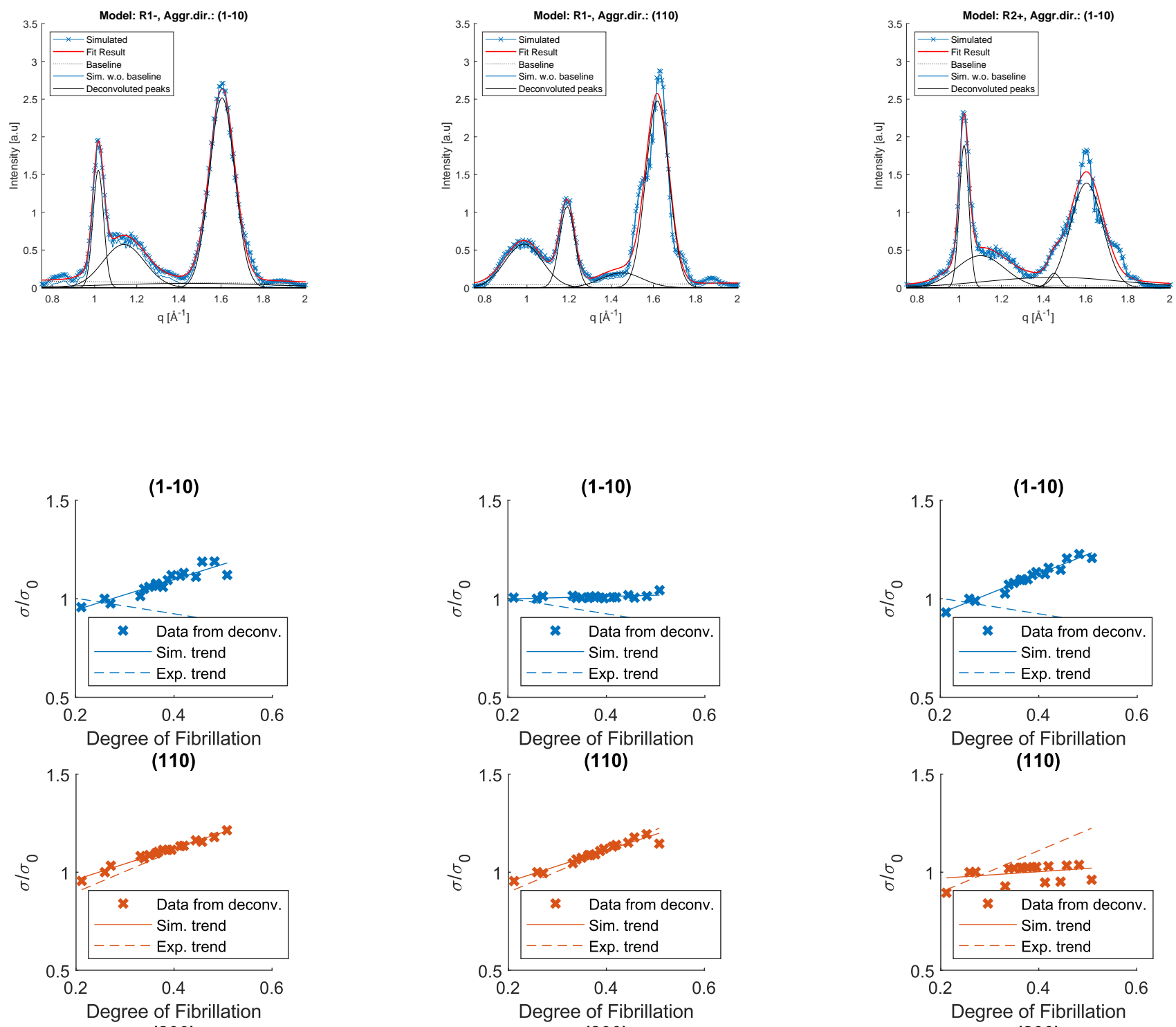

(110)
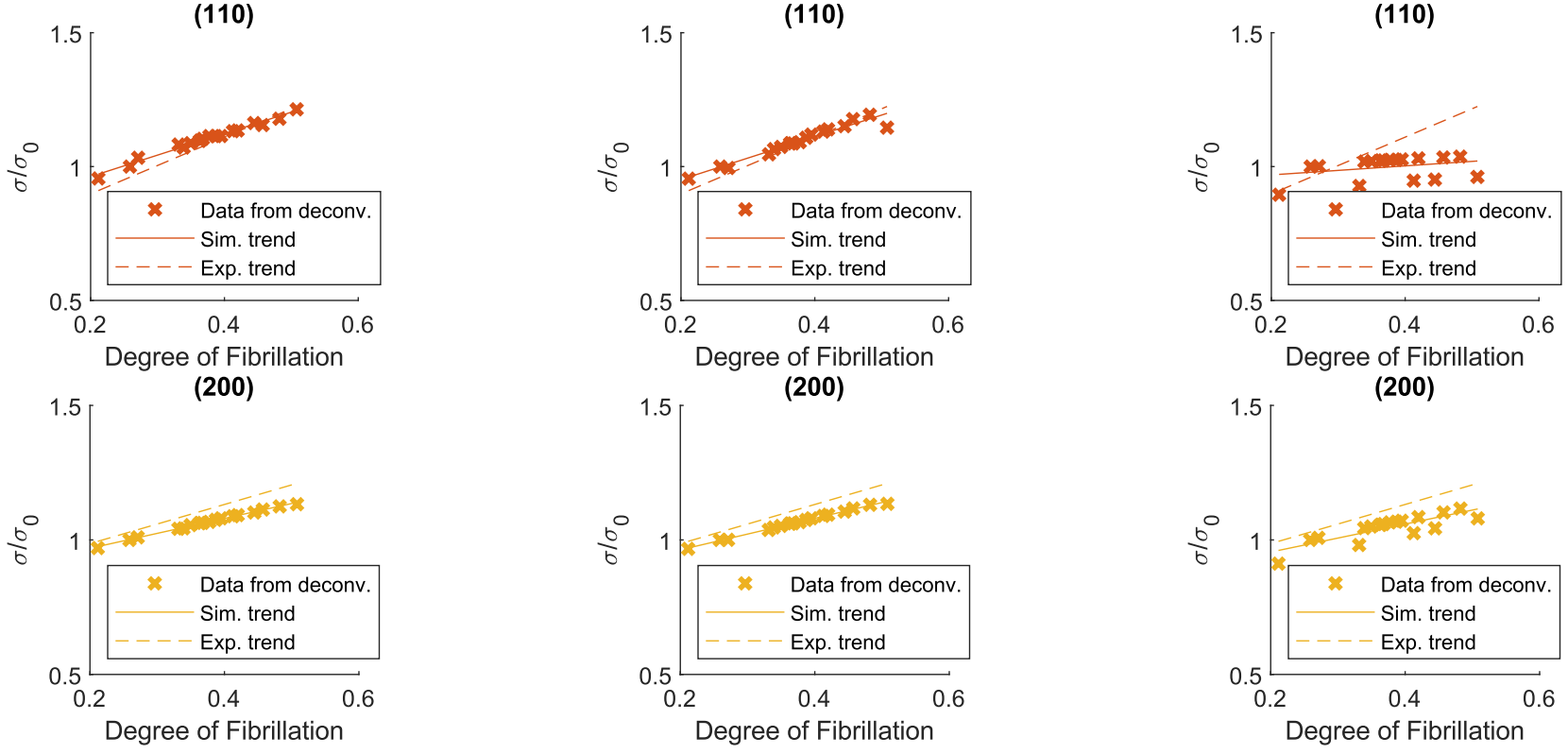

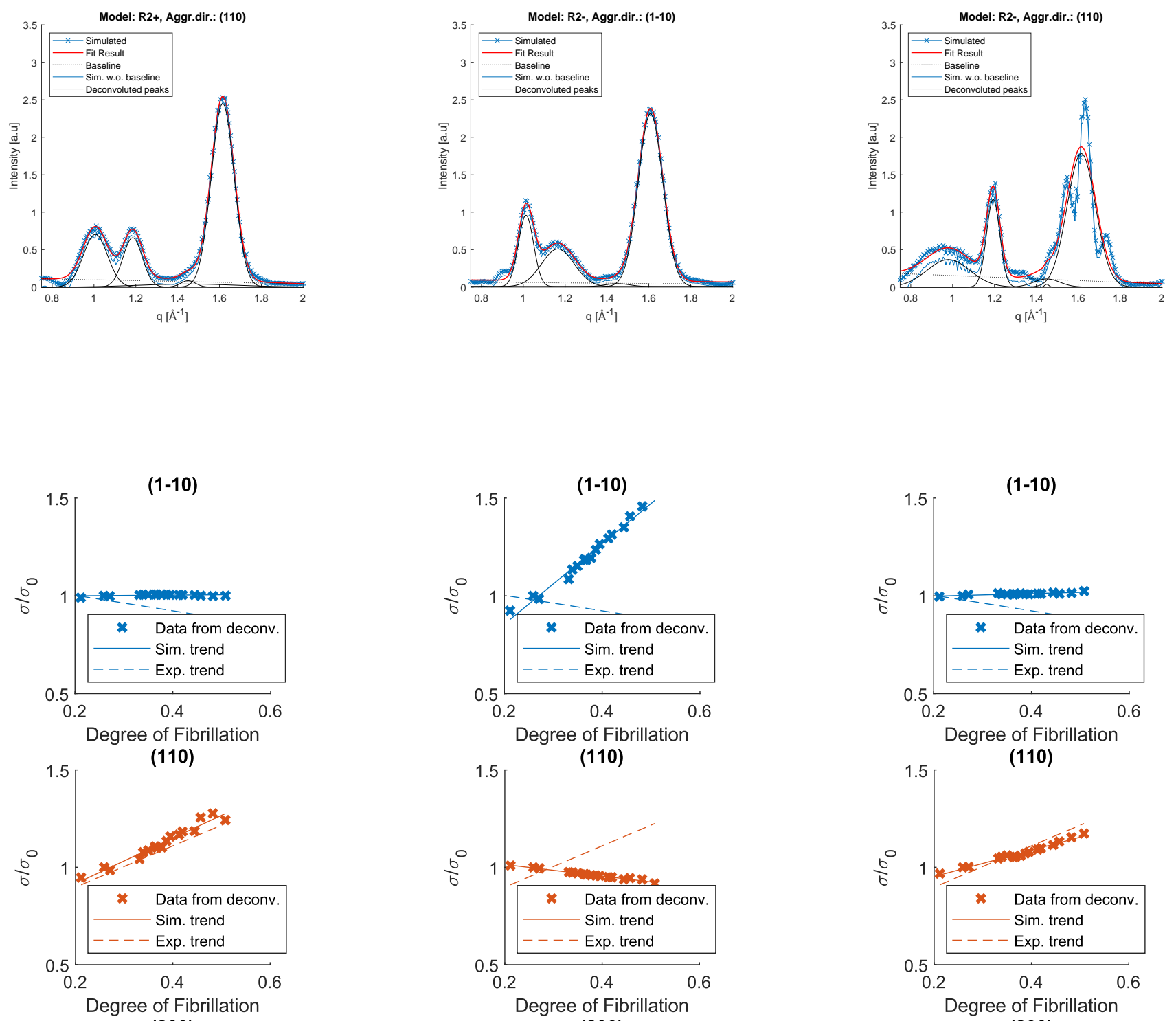

(110)
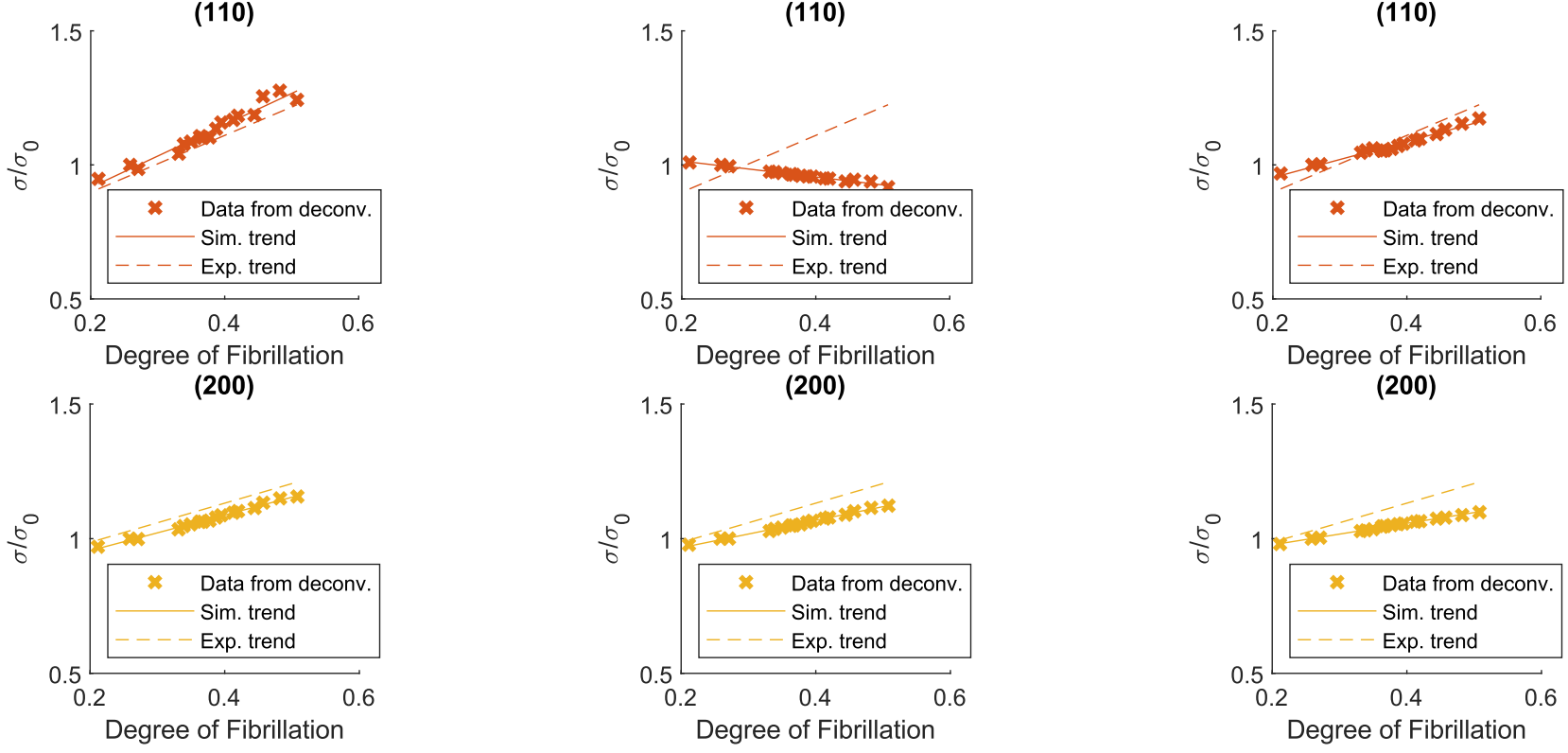

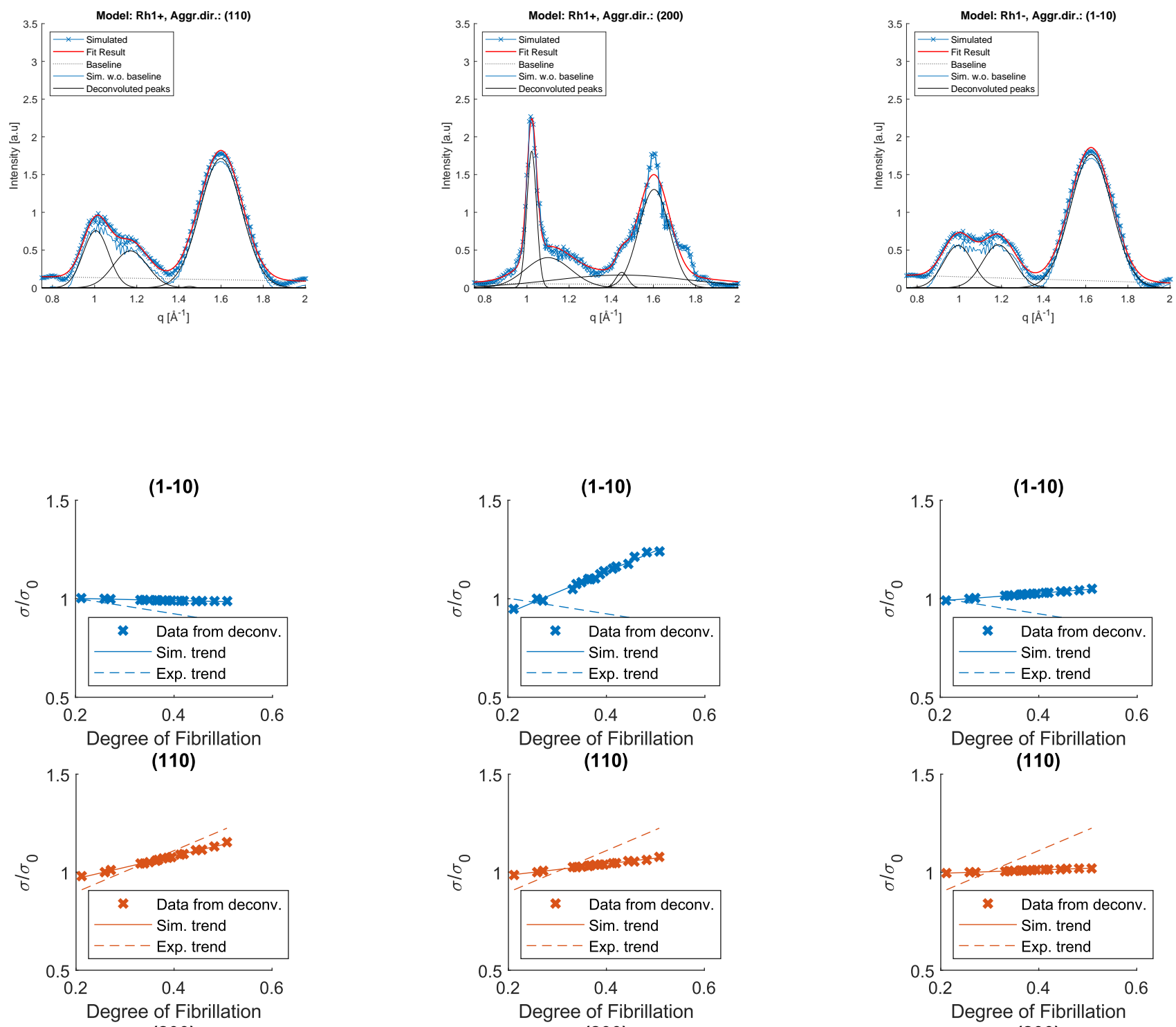

(110)
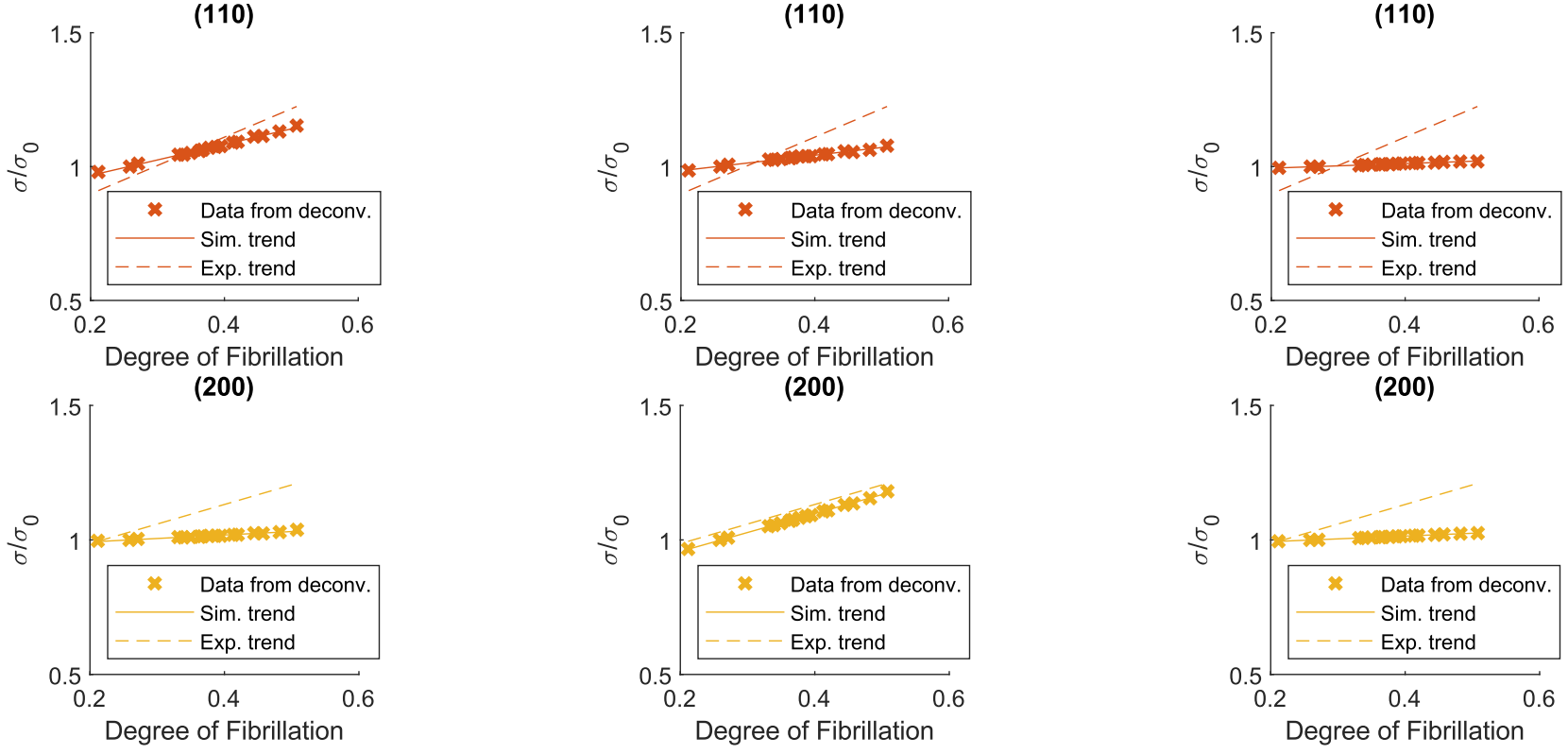

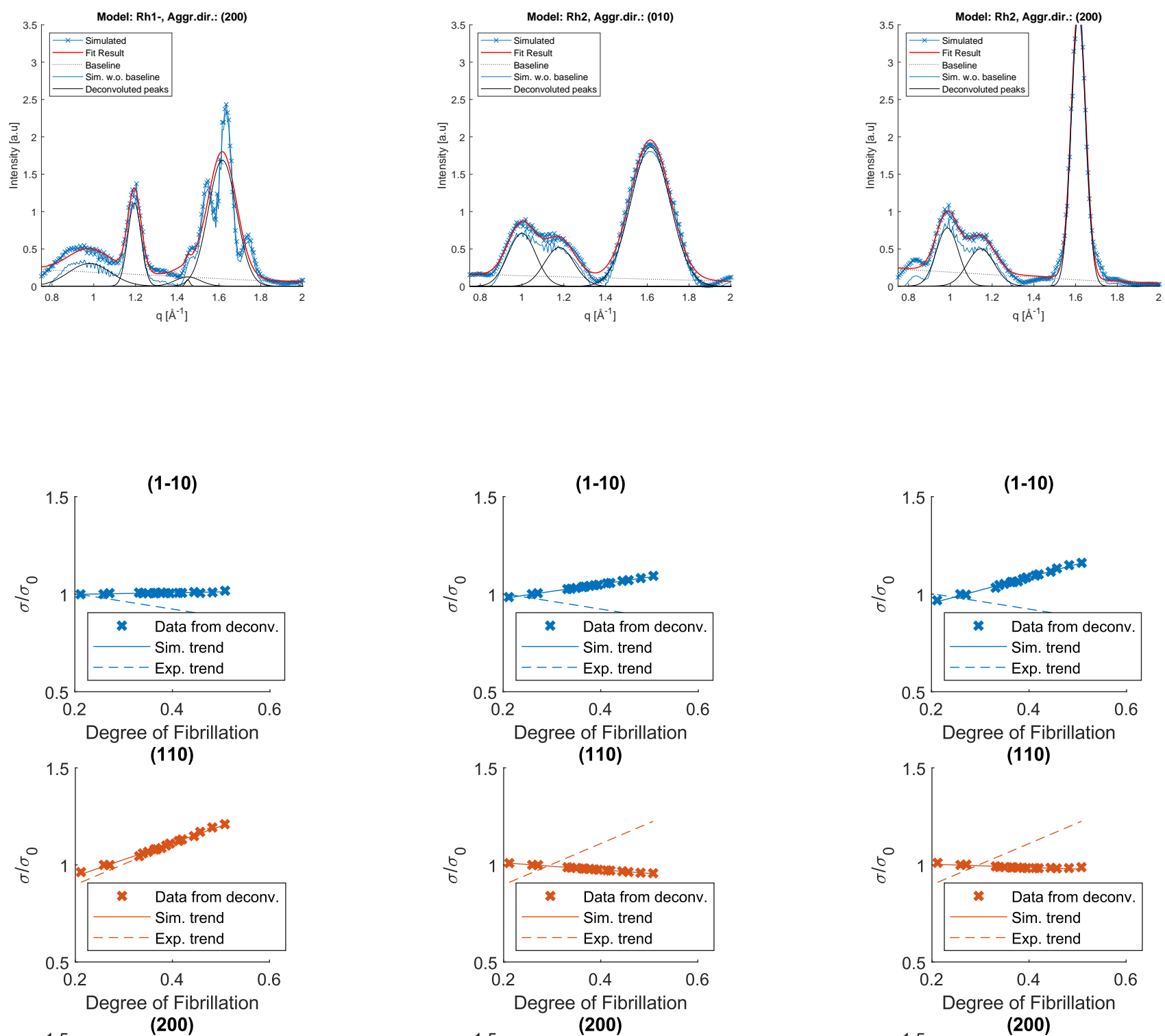

(110)
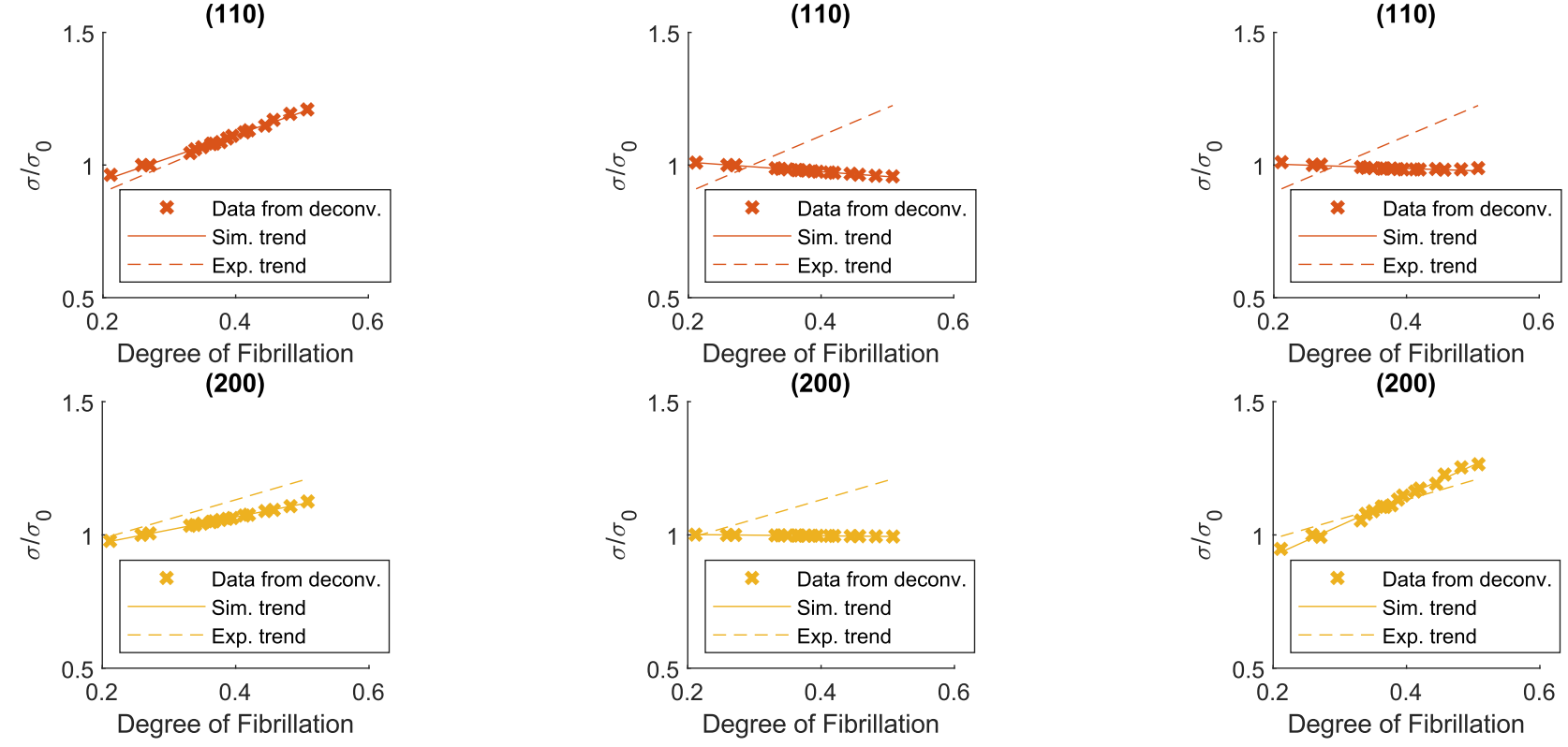